\title{
Waste Area Group 10, Operable Unit 10-08, Annual Monitoring Status Report for Fiscal Year 2009
}

February 2010 
DOE/ID-11417

Revision 0

Project No. 23368

\section{Waste Area Group 10, Operable Unit 10-08, Annual Monitoring Status Report for Fiscal Year 2009}

February 2010

Prepared for the 



\begin{abstract}
This report documents the status of Fiscal Year 2009 groundwater monitoring performed in Waste Area Group 10 at the U.S. Department of Energy Idaho National Laboratory Site, as identified in the Groundwater Monitoring and Field Sampling Plan for Operable Unit 10-08. Twelve of the fourteen required wells were sampled, and all ten required intervals from the Westbay wells were sampled. Two wells were not sampled because they were in the process of being converted into multiple-sample-interval Westbay wells by the U.S. Geological Survey. Groundwater samples were analyzed for volatile organic compounds identified on the Contract Laboratory Program target analyte list as well as metals (filtered), anions, and radionuclides (i.e., I-129, tritium, Tc-99, gross alpha, gross beta, and Sr-90).

No contaminant exceeded maximum contaminant levels in wells along the southern boundary of the Idaho National Laboratory Site or in guard wells. Iron was above its secondary maximum contaminant level of $300 \mu \mathrm{g} / \mathrm{L}$ in one well. The cause of the elevated iron concentration is uncertain. Lead was detected just below its action level. However, the zinc concentration was also elevated in these wells, and the source of the lead is probably galvanized riser pipe in the wells. Once the galvanized pipe is replaced, both lead and zinc concentrations should decline, as has been observed at other Waste Area Group 10 wells.
\end{abstract}




\section{CONTENTS}

ABSTRACT. iii

ACRONYMS vii

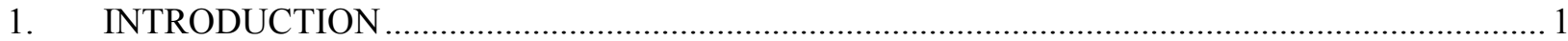

2. OPERABLE UNIT 10-08 GROUNDWATER DATA COLLECTION PERFORMED

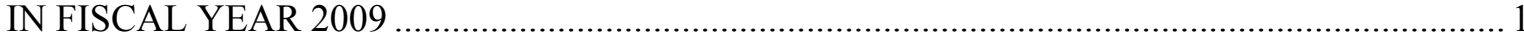

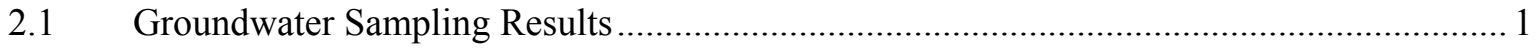

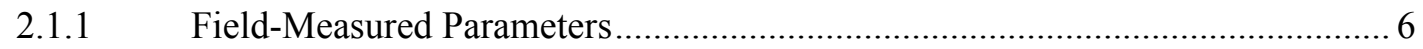

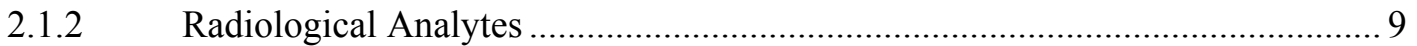

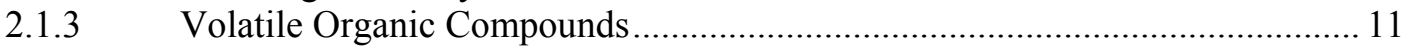

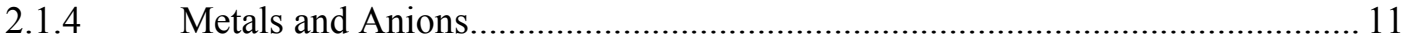

2.2 Vertical Concentration Profiles for Westbay Wells .................................................... 15

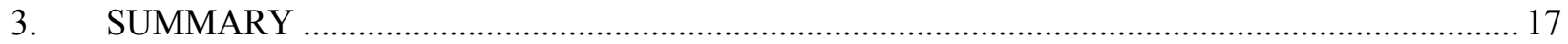

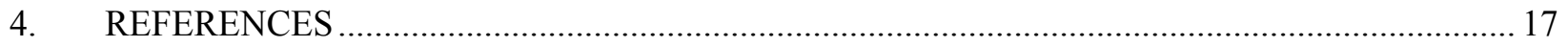

Appendix A-Analytical Results from Fiscal Year 2009 Groundwater Sampling ................................ A-1

Appendix B - Quality Assurance and Quality Control Sample Results from Fiscal Year 2009 Groundwater Sampling.

\section{FIGURES}

1. Map of the Idaho National Laboratory Site showing locations of waste area groups....................... 2

2. Location of Waste Area Group 10 boundary, guard, distal, and Westbay wells ............................. 5

3. Plot of conductivity values for Wells USGS-104, $-107,-110,-086$, and -009 ….......................... 7

4. Water-level map for the Idaho National Laboratory Site, June 2, 2007 ..................................... 8

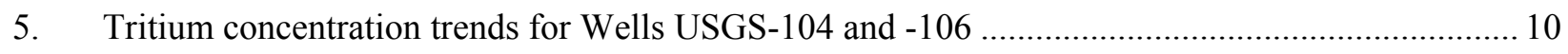

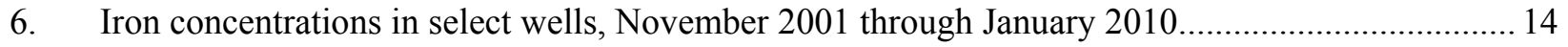

7. Zinc concentrations at selected wells that contain galvanized riser pipes, January 1999

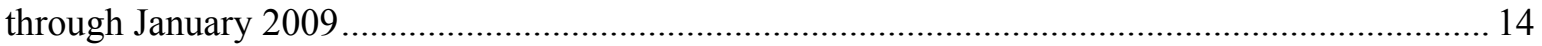

8. Nitrate trends in Wells USGS-100, -002, and -101, April 1972 through April 2007 ..................... 15

9. Vertical distribution of tritium, chloride, and sulfate in Well USGS-132 .................................. 16

10. Vertical distribution of tritium, chloride, and sulfate in Well USGS-103 ................................... 16 


\section{TABLES}

1. Construction summary for Waste Area Group 10 wells .......................................................... 3

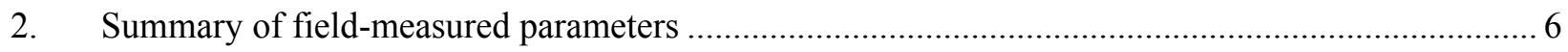

3. Sampling results for gross alpha, gross beta, and tritium for Fiscal Year 2009 ............................. 9

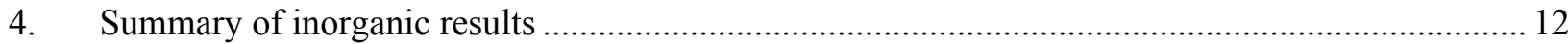




\section{ACRONYMS}

bgs below ground surface

FY fiscal year

INL Idaho National Laboratory

MCL maximum contaminant level

OU operable unit

RWMC Radioactive Waste Management Complex

USGS United States Geological Survey

WAG waste area group 


\section{Waste Area Group 10, Operable Unit 10-08, Annual Monitoring Status Report for Fiscal Year 2009}

\section{INTRODUCTION}

This report documents the status of Fiscal Year (FY) 2009 groundwater monitoring performed in Waste Area Group (WAG) 10, Operable Unit (OU) 10-08, at the U.S. Department of Energy Idaho National Laboratory (INL) Site, as identified in the Groundwater Monitoring and Field Sampling Plan for Operable Unit 10-08 (DOE-ID 2007a). The requirement for this annual report was established in the WAG 10 Remedial Investigation/Feasibility Study Work Plan (DOE-ID 2002). This document is the last monitoring report completed under the current Field Sampling Plan (DOE-ID 2007a), which governed sampling activities only until completion of the OU 10-08 Record of Decision (DOE-ID 2009a). In addition to this annual report, previous WAG 10 sampling results are summarized in the FY 2007 and FY 2008 annual reports (DOE-ID 2008a, 2009b) and the WAG 10 Remedial Investigation/Baseline Risk Assessment (DOE-ID 2008b). Future sampling activities will be described in a WAG 10 post-Record of Decision long-term monitoring plan.

WAG 10 OU 10-08 covers areas within the INL Site that are not covered by other WAGs (Figure 1). The purpose of OU 10-08 is to address concerns about the Snake River Plain Aquifer that cannot be addressed on a WAG-specific basis. Past operations at the INL Site have introduced radioactive and hazardous contaminants into the environment, and a number of these contaminants have been found in the Snake River Plain Aquifer. Potential impacts from commingled groundwater plumes from INL Site activities were investigated as part of the WAG 10 Remedial Investigation/Baseline Risk Assessment (DOE-ID 2008b).

WAG 10 was created in accordance with the Federal Facility Agreement and Consent Order for the Idaho National Engineering Laboratory (DOE-ID 1991) and the "Comprehensive Environmental Response, Compensation, and Liability Act of 1980" (42 USC $\S 9601$ et seq.), as amended by the "Superfund Amendments and Reauthorization Act of 1986 (SARA)" (Public Law 99-499).

\section{OPERABLE UNIT 10-08 GROUNDWATER DATA COLLECTION PERFORMED IN FISCAL YEAR 2009}

WAG 10 field sampling activities are planned and approved by the Agencies (i.e., U.S. Department of Energy, U.S. Environmental Protection Agency, and Idaho Department of Environmental Quality) through preparation of formal work planning documents (e.g., OU 10-08 Field Sampling Plan [DOE-ID 2007a]). Groundwater monitoring tasks performed in FY 2009 comprised collecting groundwater quality data and presenting the results in this report.

\subsection{Groundwater Sampling Results}

Wells sampled for WAG 10 are divided into five groups: boundary, guard, baseline, distal, and Westbay. Table 1 provides information about construction of WAG 10 wells, and Figure 2 shows well locations. For FY 2009, the OU 10-08 Field Sampling Plan called for sampling 14 boundary and guard wells, three sampling intervals from Westbay Well USGS-132, and seven sampling intervals from Westbay Well USGS-103. Wells USGS-105 and -108 were not sampled because the U.S. Geological Survey (USGS) was in the process of installing a Westbay system in them. Groundwater samples 


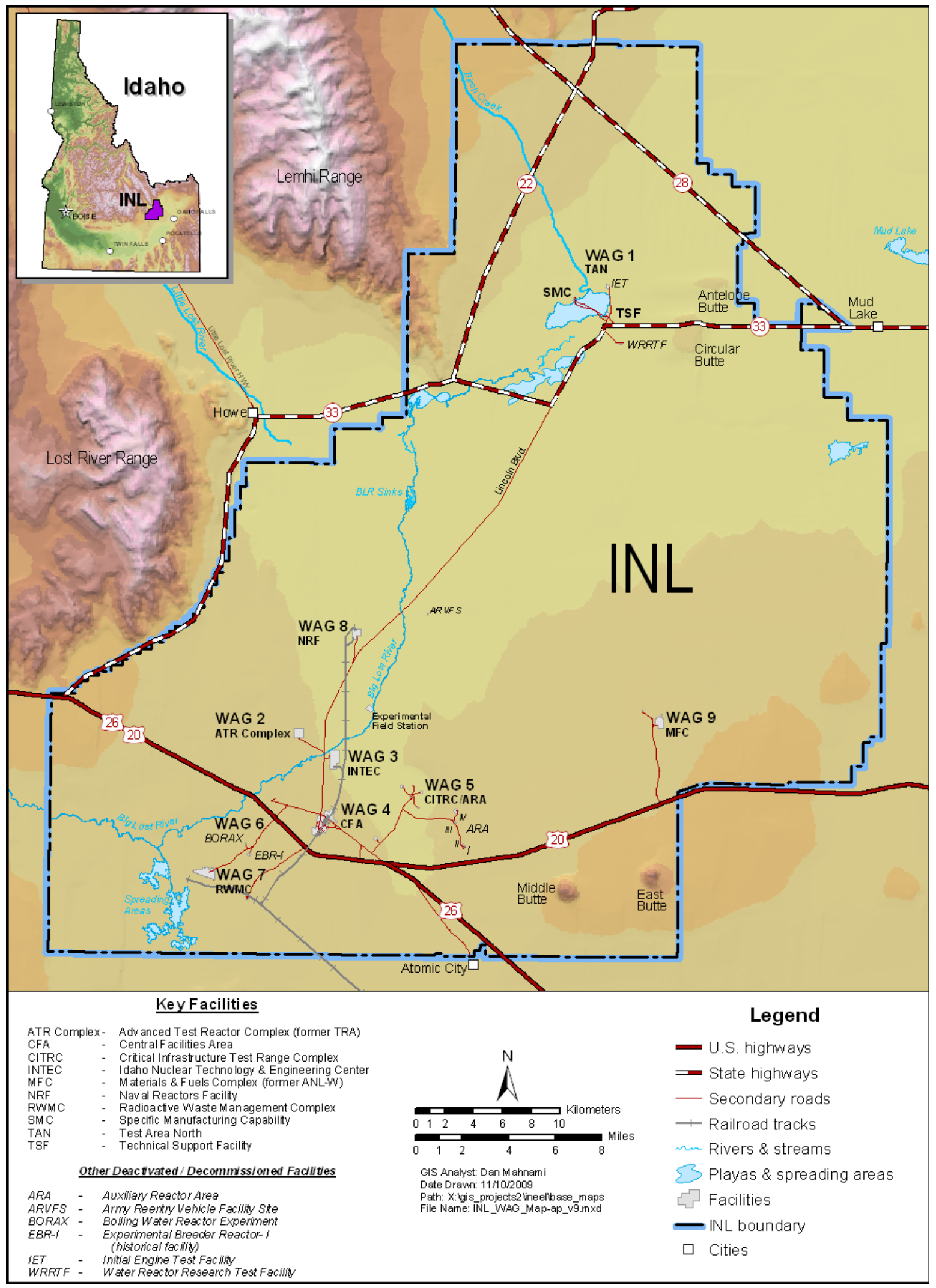

Figure 1. Map of the Idaho National Laboratory Site showing locations of waste area groups. 
Table 1. Construction summary for Waste Area Group 10 wells.

\begin{tabular}{|c|c|c|c|c|}
\hline $\begin{array}{c}\text { Well } \\
\text { Identifier }^{\mathrm{a}} \\
\end{array}$ & Well Name & $\begin{array}{l}\text { Screened or Open Hole } \\
(\mathrm{ft} \text { bgs })\end{array}$ & $\begin{array}{l}\text { Pump Depth } \\
\text { (ft bgs) })^{b}\end{array}$ & $\begin{array}{l}\text { Approximate } \\
\text { Depth to Water } \\
\text { (ft bgs) }\end{array}$ \\
\hline \multicolumn{5}{|c|}{ Boundary Wells } \\
\hline 450 & USGS-001 & $600-630$ perforated & 612 & 588 \\
\hline 458 & USGS-009 & $620-650$ perforated & 635 & 607 \\
\hline 535 & USGS-086 & 48-691 open hole & 678 & 649 \\
\hline 550 & USGS-101 & $750-865$ perforated & 790 & 771 \\
\hline 554 & USGS-105 & $400-800$ open hole & 700 & 670 \\
\hline 557 & USGS-108 & 400-760 open hole & 637 & 609 \\
\hline 558 & USGS-109 & $600-800$ open hole & 656 & 621 \\
\hline 559 & USGS-110 & 580-780 open hole & 612 & 566 \\
\hline \multicolumn{5}{|l|}{ Guard Wells } \\
\hline 184 & Highway 3 & $680-750$ perforated & 567 & 538 \\
\hline 451 & USGS-002 & $675-696$ perforated & 683 & 659 \\
\hline 549 & USGS-100 & $662-750$ open hole & 703 & 686 \\
\hline 553 & USGS-104 & 550-700 open hole & 592 & 555 \\
\hline 555 & USGS-106 & $605-760$ open hole & 609 & 584 \\
\hline 556 & USGS-107 & 270-690 open hole & 531 & 477 \\
\hline \multicolumn{5}{|c|}{ Baseline Wells } \\
\hline 453 & USGS-004 & $\begin{array}{l}\text { 285-315 perforated } \\
322-553 \text { open hole }\end{array}$ & 303 & 251 \\
\hline 457 & USGS-008 & $782-812$ perforated & 801 & 766 \\
\hline 468 & USGS-019 & 289-305 perforated & 323 & 276 \\
\hline 475 & USGS-026 & $232-266.5$ perforated & 255 & 212 \\
\hline 476 & USGS-027 & $\begin{array}{l}250-260 \text { perforated } \\
298-308 \text { perforated }\end{array}$ & 262 & 228 \\
\hline 1346 & USGS-126B & 400-452 open hole & 420 & 408 \\
\hline 147 & DH-1B & $380-400$ open hole & 357 & 268 \\
\hline 250 & $\mathrm{P} \& \mathrm{~W}-3$ & $322-401$ perforated & 340 & 304 \\
\hline \multicolumn{5}{|l|}{ Distal Wells } \\
\hline 460 & USGS-011 & $672.5-703.8$ perforated & 687 & 658 \\
\hline 463 & USGS-014 & $720-746$ perforated & 739 & 722 \\
\hline 987 & USGS-124 & $750-800$ slotted & Not available & 688 \\
\hline 988 & USGS-125 & 620-774 slotted & 700 & 634 \\
\hline \multicolumn{5}{|c|}{ Westbay Wells ${ }^{d}$} \\
\hline 2050 & MIDDLE-2050A & Sampling port-525.2 & NA & NA \\
\hline 2050 & MIDDLE-2050A & Sampling port-652.1 & NA & NA \\
\hline 2050 & MIDDLE-2050A & Sampling port-800.5 & NA & NA \\
\hline 2050 & MIDDLE-2050A & Sampling port-1,007.9 & NA & NA \\
\hline 2050 & MIDDLE-2050A & Sampling port-1,189.1 & NA & NA \\
\hline 2051 & MIDDLE-2051 & Sampling port-593.7 & NA & NA \\
\hline 2051 & MIDDLE-2051 & Sampling port-760.4 & NA & NA \\
\hline 2051 & MIDDLE-2051 & Sampling port- -838.1 & NA & NA \\
\hline
\end{tabular}


Table 1. (continued).

\begin{tabular}{|c|c|c|c|c|}
\hline $\begin{array}{c}\text { Well } \\
\text { Identifier }^{\mathrm{a}}\end{array}$ & Well Name & $\begin{array}{l}\text { Screened or Open Hole } \\
(\mathrm{ft} \text { bgs) }\end{array}$ & $\begin{array}{l}\text { Pump Depth } \\
(\mathrm{ft} \mathrm{bgs})^{\mathrm{b}}\end{array}$ & $\begin{array}{l}\text { Approximate } \\
\text { Depth to Water } \\
\text { (ft bgs) }\end{array}$ \\
\hline 2051 & MIDDLE-2051 & Sampling port-1,102.8 & NA & NA \\
\hline 2051 & MIDDLE-2051 & Sampling port-1,152.8 & NA & NA \\
\hline 552 & USGS-103 & Sampling port-681.9 & NA & NA \\
\hline 552 & USGS-103 & Sampling port-805.2 & NA & NA \\
\hline 552 & USGS-103 & Sampling port-913.8 & NA & NA \\
\hline 552 & USGS-103 & Sampling port-999.4 & NA & NA \\
\hline 552 & USGS-103 & Sampling port-1,095.1 & NA & NA \\
\hline 552 & USGS-103 & Sampling port-1,220.4 & NA & NA \\
\hline 552 & USGS-103 & Sampling port-1,269.4 & NA & NA \\
\hline 2029 & USGS-132 & Sampling port-646.7 & NA & NA \\
\hline 2029 & USGS-132 & Sampling port-774.2 & NA & NA \\
\hline 2029 & USGS-132 & Sampling port-836 & NA & NA \\
\hline 2029 & USGS-132 & Sampling port-927.5 & NA & NA \\
\hline 2029 & USGS-132 & Sampling port-1,020.5 & NA & NA \\
\hline 2029 & USGS-132 & Sampling port-1,182.5 & NA & NA \\
\hline \multicolumn{5}{|c|}{$\begin{array}{l}\text { a. Well identifier is from the Environmental Data Warehouse (ICP, 2009, Environmental Data Warehouse, http://icpweb/edw2/, Idaho } \\
\text { Cleanup Project, Web page visited December 1, 2009. [Note: This Web page is on the ICP intranet and not publicly available.]) } \\
\text { b. Pump depth is the depth to the top of the pump. } \\
\text { c. Well USGS-124 was in use, and the pump depth could not be determined when the well was last sampled. } \\
\text { d. Sampling depths for Wells MIDDLE-2050A and MIDDLE-2051 were corrected from those reported in previous monitoring reports } \\
\text { (DOE-ID 2006, 2007b) to reflect as-built depths. }\end{array}$} \\
\hline \multicolumn{5}{|c|}{$\begin{array}{l}\text { below ground surface } \\
\text { not applicable }\end{array}$} \\
\hline
\end{tabular}




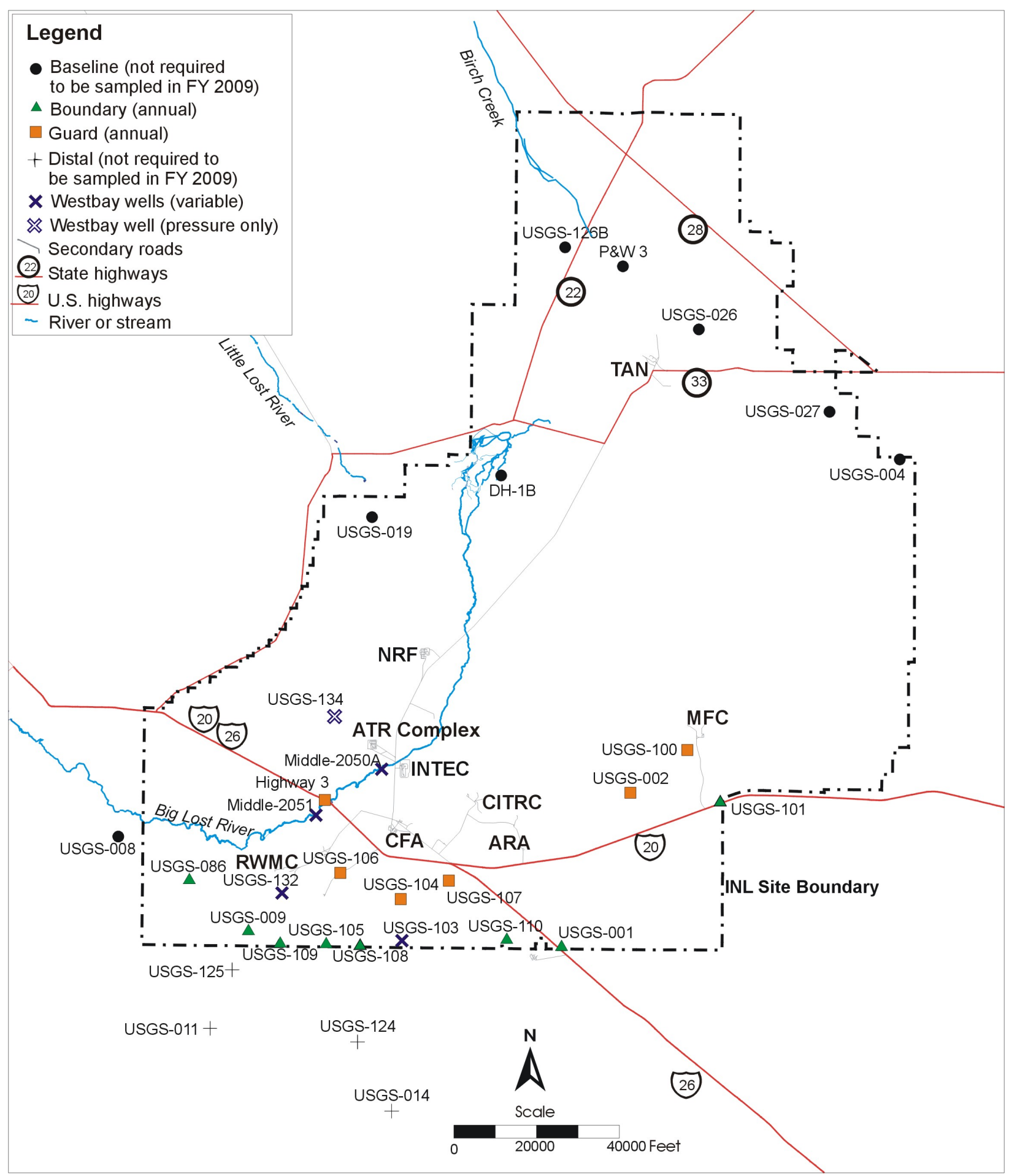

Figure 2. Location of Waste Area Group 10 boundary, guard, distal, and Westbay wells. 
collected during June through July 2009 were analyzed for volatile organic compounds from the Contract Laboratory Program target analyte list, metals (filtered), anions, alkalinity, nitrate, and radionuclides (i.e., I-129, tritium, Tc-99, gross alpha, gross beta, and Sr-90). Appendix A provides full analytical results for each well, and Appendix B discusses the quality assurance and quality control sample results.

\subsubsection{Field-Measured Parameters}

Table 2 provides summary data for field-measured parameters (including temperature, $\mathrm{pH}$, conductivity, and dissolved oxygen) and approximate water level at the time of sampling. Data for field parameters are included because abnormal $\mathrm{pH}$ (high or low) and high conductivity values can be used as indicators of contamination. The $\mathrm{pH}$, temperature, conductivity, and dissolved-oxygen measurements in FY 2009 are consistent with previous measurements. The $\mathrm{pH}$ data indicate slightly alkaline conditions with a $\mathrm{pH}$ range of 7.81 to 8.45 . Conductivity varies from 0.300 to $0.424 \mathrm{mmhos} / \mathrm{cm}$, with the highest conductivity reading occurring in the uppermost interval from Well USGS-132, located south of the Radioactive Waste Management Complex (RWMC). Figure 3 shows conductivity trends for select wells. Dissolved-oxygen data indicate oxidizing conditions exist throughout the aquifer. Dissolved oxygen was measured with a flow-through cell for all the wells sampled. It is unlikely that dissolved-oxygen concentrations changed more than marginally from the in situ conditions, given the pump rates used to collect samples. Water-level data were collected from wells that were sampled using an e-line, in accordance with the latest procedure. These data are presented in Table 2. Maps showing Site-wide water levels are presented in the annual reports for FY 2004, 2005, and 2007 (DOE-ID 2005, 2006, 2008a). The water level map for June 2, 2007, is shown in Figure 4.

Table 2. Summary of field-measured parameters.

\begin{tabular}{|c|c|c|c|c|c|c|}
\hline Well Name & $\begin{array}{l}\text { Water Level } \\
(\mathrm{ft} \text { bgs })^{\mathrm{a}}\end{array}$ & Date Sampled & $\begin{array}{l}\text { Temp. } \\
\left({ }^{\circ} \mathrm{C}\right)^{\mathrm{b}}\end{array}$ & $\mathrm{pH}$ & $\begin{array}{c}\text { Specific } \\
\text { Conductivity } \\
(\mathrm{mmhos} / \mathrm{cm})\end{array}$ & $\begin{array}{l}\text { Dissolved } \\
\text { Oxygen } \\
(\mathrm{mg} / \mathrm{L})^{\mathrm{c}}\end{array}$ \\
\hline \multicolumn{7}{|l|}{ Boundary Wells } \\
\hline USGS-001 & 594.97 & $6 / 03 / 2009$ & 14.21 & 8.01 & 0.333 & 7.3 \\
\hline USGS-009 & 615.18 & $6 / 17 / 2009$ & 1194 & 8.03 & 0.355 & 4.74 \\
\hline USGS-086 & 656.54 & $6 / 16 / 2009$ & 10.32 & 8.24 & 0.325 & 6.78 \\
\hline USGS-101 & 781.14 & $6 / 03 / 2009$ & 14.04 & 8.06 & 0.300 & 5.25 \\
\hline USGS-105 & NS & NS & NS & NS & NS & NS \\
\hline USGS-108 & NS & NS & NS & NS & NS & NS \\
\hline USGS-109 & $627.13^{\mathrm{d}}$ & $6 / 10 / 2009$ & 13.56 & 7.96 & 0.374 & 5.84 \\
\hline USGS-110 & 570.45 & $6 / 02 / 2009$ & 14.35 & 7.81 & 0.384 & 2.13 \\
\hline \multicolumn{7}{|l|}{ Guard Wells } \\
\hline USGS-100 & 687.17 & $6 / 23 / 2009$ & 13.48 & 8.09 & 0.366 & 6.11 \\
\hline Highway 3 & $\mathrm{NM}^{\mathrm{e}}$ & $6 / 09 / 2009$ & 11.58 & 7.89 & 0.351 & 6.82 \\
\hline USGS-002 & 669.56 & $6 / 22 / 2009$ & 13.48 & 7.97 & 0.366 & 6.11 \\
\hline USGS-104 & 562.89 & $6 / 15 / 2009$ & 12.06 & 7.98 & 0.317 & 5.47 \\
\hline USGS-106 & 593.13 & $6 / 10 / 2009$ & 13.83 & 7.91 & 0.396 & 5.94 \\
\hline USGS-107 & 486.43 & $6 / 10 / 2009$ & 15.35 & 8.01 & 0.409 & 5.47 \\
\hline Westbay Wells & Sampling Depth & & & & & \\
\hline USGS-103 & 681.9 & $7 / 01 / 2009$ & 16.09 & 8.45 & 0.309 & 3.24 \\
\hline USGS-103 & 805.2 & $7 / 21 / 2009$ & 15.19 & 8.09 & 0.351 & 10.85 \\
\hline USGS-103 & 913.8 & $7 / 02-7 / 07 / 2009$ & 14.25 & 8.01 & 0.322 & 11.71 \\
\hline USGS-103 & 999.4 & $7 / 07-7 / 08 / 2009$ & 13.45 & 7.99 & 0.350 & 9.18 \\
\hline
\end{tabular}


Table 2. (continued).

\begin{tabular}{ccccccc}
\hline Well Name & $\begin{array}{c}\text { Water Level } \\
(\mathrm{ft} \mathrm{bgs})^{\mathrm{a}}\end{array}$ & Date Sampled & $\begin{array}{c}\text { Temp. } \\
\left({ }^{\circ} \mathrm{C}\right)^{\mathrm{b}}\end{array}$ & $\mathrm{pH}$ & $\begin{array}{c}\text { Specific } \\
\text { Conductivity } \\
(\mathrm{mmhos} / \mathrm{cm})\end{array}$ & $\begin{array}{c}\text { Dissolved } \\
\text { Oxygen } \\
(\mathrm{mg} / \mathrm{L})^{\mathrm{c}}\end{array}$ \\
\hline USGS-103 & $1,095.1$ & $7 / 08 / 2009$ & 14.49 & 8.29 & 0.369 & 8.37 \\
USGS-103 & $1,220.3$ & $7 / 08-7 / 12 / 2009$ & 14.40 & 8.12 & 0.371 & 10.04 \\
USGS-103 & $1,269.4$ & $7 / 13 / 2009$ & $\mathrm{NM}$ & $\mathrm{NM}$ & $\mathrm{NM}$ & $\mathrm{NM}$ \\
USGS-132 & 646.7 & $6 / 24-6 / 25 / 2009$ & 13.77 & 8.16 & 0.424 & 4.40 \\
USGS-132 & 774.2 & $6 / 25-6 / 29 / 2009$ & 15.36 & 8.15 & 0.369 & 7.69 \\
USGS-132 & 836 & $6 / 29-6 / 30 / 2009$ & 14.36 & 8.14 & 0.362 & 6.80 \\
\hline
\end{tabular}

a. Water level measurement before sampling.

b. Temperature, dissolved oxygen, conductivity, and $\mathrm{pH}$ were measured using a flow-through cell.

c. Dissolved-oxygen sensors were not functioning in some wells and were replaced.

d. Water level in Well USGS-109 was not measured because the water level indicator was not functioning at the time of sampling.

e. Water level in the Highway 3 well was not measured because there is no water-level access line.

\begin{tabular}{ll} 
bgs & below ground surface \\
NM & not measured \\
NS & not sampled \\
\hline
\end{tabular}

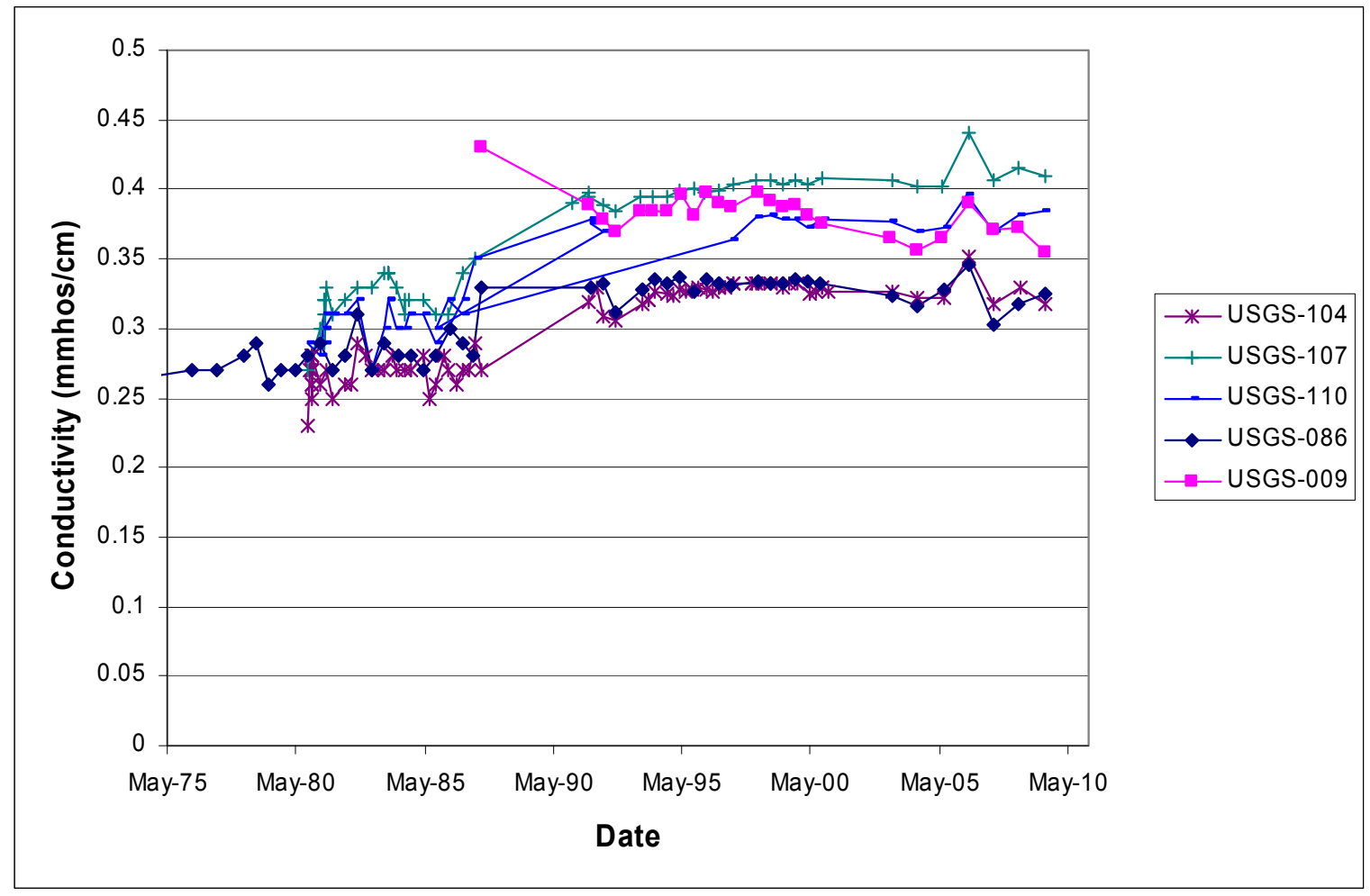

Figure 3. Plot of conductivity values for Wells USGS-104, -107, -110, -086, and -009. 


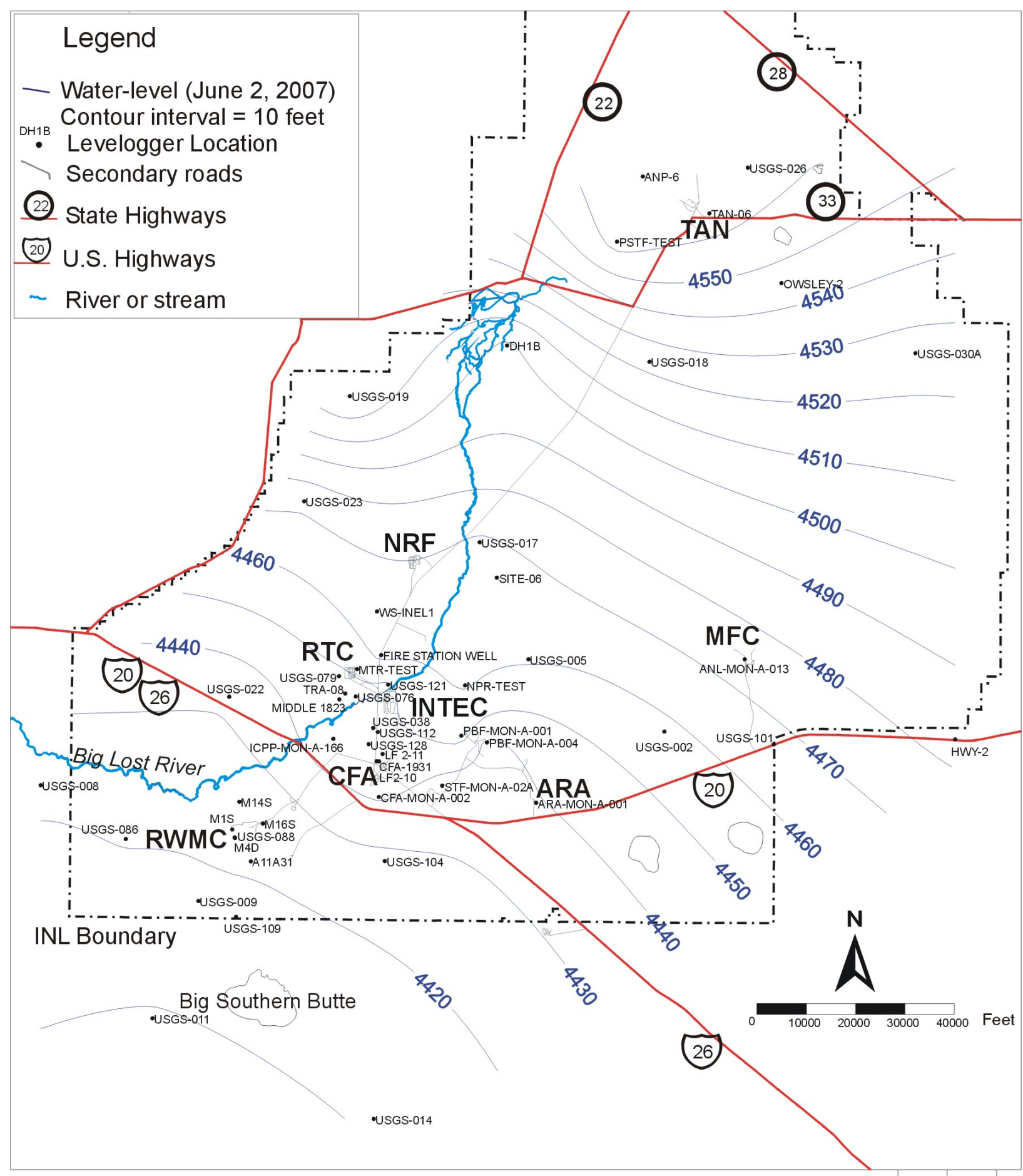

Figure 4. Water-level map for the Idaho National Laboratory Site, June 2, 2007 (DOE-ID-2008a). 


\subsubsection{Radiological Analytes}

Radiological analytes detected in boundary and guard wells included gross alpha, gross beta, and tritium (Table 3). These analytes measured lower than their respective maximum contaminant levels (MCLs). Concentrations of gross alpha and gross beta in WAG 10 wells were at background levels, based on background values from Knobel, Orr, and Cecil (1992). Iodine-129, Tc-99, and Sr-90 were not detected in any samples. Tritium was detected in two wells (i.e., Wells USGS-104 and -106) and in two intervals from Westbay Well USGS-103. Wells USGS-104 and -106 have a history of tritium detections with both wells exhibiting a downward trend in tritium concentrations (Figure 5).

Tritium concentrations in Well USGS-104 are currently less than $800 \mathrm{pCi} / \mathrm{L}$ and considerably less than the MCL of 20,000 pCi/L (Table 3). Tritium was detected in two intervals from Well USGS-103 at depths of 1,095.1 ft (i.e., $360 \mathrm{pCi} / \mathrm{L}$ ) and 1,269.4 ft (i.e., $437 \mathrm{pCi} / \mathrm{L}$ ) below ground surface (bgs).

Both detections of tritium in Well USGS-103 are close to the average minimum detectable activity of $311 \mathrm{pCi} / \mathrm{L}$ for the seven samples from Well USGS-103 (Table 3).

Table 3. Sampling results for gross alpha, gross beta, and tritium for Fiscal Year 2009.

\begin{tabular}{|c|c|c|c|c|c|c|c|c|c|c|}
\hline \multirow[b]{2}{*}{ Well } & & \multicolumn{3}{|c|}{$\begin{array}{c}\text { Gross Alpha } \\
(\mathrm{MCL}=15 \mathrm{pCi} / \mathrm{L})\end{array}$} & \multicolumn{3}{|c|}{$\begin{array}{c}\text { Gross Beta } \\
(\mathrm{MCL}=4 \mathrm{mrem} / \mathrm{year})^{\mathrm{a}}\end{array}$} & \multicolumn{3}{|c|}{$\begin{array}{c}\text { Tritium } \\
(\mathrm{MCL}=20,000 \mathrm{pCi} / \mathrm{L})\end{array}$} \\
\hline & & $\mathrm{pCi} / \mathrm{L}$ & $\pm^{\mathrm{b}}$ & $\begin{array}{c}\text { Validation } \\
\text { Flag }^{\mathrm{c}}\end{array}$ & $\mathrm{pCi} / \mathrm{L}$ & $\pm^{\mathrm{b}}$ & $\begin{array}{c}\text { Validation } \\
\text { Flag }^{\mathrm{c}} \\
\end{array}$ & $\mathrm{pCi} / \mathrm{L}$ & $\pm^{\mathrm{b}}$ & $\begin{array}{c}\text { Validation } \\
\text { Flag }^{\mathrm{c}}\end{array}$ \\
\hline \multicolumn{11}{|c|}{ Guard Wells } \\
\hline Highway 3 & & 1.92 & 0.675 & $\mathrm{~J}$ & 1.62 & 0.562 & $\mathrm{~J}$ & $-71.3^{\mathrm{d}}$ & 85.9 & $\mathrm{U}$ \\
\hline USGS-002 & & 1.36 & 0.676 & UJ & 3.95 & 1.09 & & -6.38 & 106 & $\mathrm{U}$ \\
\hline USGS-100 & & 2.04 & 0.704 & $\mathrm{~J}$ & 2.57 & 0.648 & & 25.8 & 109 & $\mathrm{U}$ \\
\hline USGS-104 & & -0.159 & 0.333 & $\mathrm{U}$ & 1.62 & 0.53 & & 753 & 132 & \\
\hline USGS-104 & Dup & 4.62 & 1.23 & & 3.35 & 0.995 & & 567 & 118 & \\
\hline USGS-106 & & 1.88 & 0.757 & UJ & 3.42 & 0.876 & & 467 & 111 & \\
\hline USGS-106 & Dup & 2.39 & 0.82 & $\mathrm{~J}$ & 2.59 & 0.753 & & 435 & 109 & \\
\hline USGS-107 & & 3.14 & 0.985 & & 3.04 & 1.06 & UJ & 0 & 87.7 & $\mathrm{U}$ \\
\hline \multicolumn{11}{|c|}{ INL Site Boundary Wells } \\
\hline USGS-001 & & 1.3 & 0.576 & UJ & 4.01 & 0.773 & & -60.4 & 86.1 & $\mathrm{U}$ \\
\hline USGS-009 & & 2.4 & 0.911 & $\mathrm{~J}$ & 6.42 & 1.21 & & 47.3 & 89.4 & $\mathrm{U}$ \\
\hline USGS-086 & & 4.22 & 0.849 & & 6.3 & 0.882 & & -114 & 84.2 & $\mathrm{U}$ \\
\hline USGS-101 & & 0.731 & 0.418 & $\mathrm{U}$ & 6.25 & 1.17 & & -64.5 & 85.7 & $\mathrm{U}$ \\
\hline USGS-105 & & NS & NS & NS & NS & NS & NS & NS & NS & NS \\
\hline USGS-108 & & NS & NS & NS & NS & NS & NS & NS & NS & NS \\
\hline USGS-109 & & 0.454 & 0.496 & $\mathrm{U}$ & 3.79 & 0.859 & & 101 & 91.1 & $\mathrm{U}$ \\
\hline USGS-110 & & 2.02 & 0.741 & $\mathrm{~J}$ & 4.23 & 1.03 & & 0 & 88.3 & $\mathrm{U}$ \\
\hline
\end{tabular}


Table 3. (continued).

\begin{tabular}{|c|c|c|c|c|c|c|c|c|c|c|}
\hline \multirow{2}{*}{\multicolumn{2}{|c|}{ Well }} & \multicolumn{3}{|c|}{$\begin{array}{c}\text { Gross Alpha } \\
(\mathrm{MCL}=15 \mathrm{pCi} / \mathrm{L})\end{array}$} & \multicolumn{3}{|c|}{$\begin{array}{c}\text { Gross Beta } \\
(\mathrm{MCL}=4 \mathrm{mrem} / \text { year })^{\mathrm{a}}\end{array}$} & \multicolumn{3}{|c|}{$\begin{array}{c}\text { Tritium } \\
(\mathrm{MCL}=20,000 \mathrm{pCi} / \mathrm{L})\end{array}$} \\
\hline & & $\mathrm{pCi} / \mathrm{L}$ & $\pm^{\mathrm{b}}$ & $\begin{array}{c}\text { Validation } \\
\text { Flag }^{c}\end{array}$ & $\mathrm{pCi} / \mathrm{L}$ & $\pm^{\mathrm{b}}$ & $\begin{array}{c}\text { Validation } \\
\text { Flag }^{c}\end{array}$ & $\mathrm{pCi} / \mathrm{L}$ & $\pm^{\mathrm{b}}$ & $\begin{array}{l}\text { Validation } \\
\text { Flag }^{c}\end{array}$ \\
\hline \multicolumn{11}{|c|}{ Westbay Wells and Depths } \\
\hline \multicolumn{11}{|c|}{ USGS-103 } \\
\hline & 681.9 & 3.84 & 0.953 & & 2.62 & 0.671 & & -28 & 106 & $\mathrm{U}$ \\
\hline & 805.2 & 0.924 & 0.419 & UJ & 4.31 & 0.697 & $\mathrm{~J}$ & 70.6 & 106 & $\mathrm{U}$ \\
\hline & 913.8 & 1.53 & 0.545 & UJ & 3.32 & 0.738 & $\mathrm{~J}$ & 122 & 87 & $\mathrm{U}$ \\
\hline & 999.4 & 1.88 & 0.571 & UJ & 2.73 & 0.596 & UJ & 107 & 86.4 & $\mathrm{U}$ \\
\hline & $1,095.1$ & 1.07 & 0.449 & UJ & 3.32 & 0.594 & $\mathrm{~J}$ & 360 & 99.9 & \\
\hline & $1,220.2$ & 0.138 & 0.323 & $\mathrm{U}$ & 2 & 0.394 & UJ & 250 & 93.4 & UJ \\
\hline & $1,269.4$ & 1.87 & 0.62 & UJ & 1.52 & 0.266 & UJ & 437 & 105 & \\
\hline \multicolumn{11}{|c|}{ USGS-132 } \\
\hline & 646.7 & 2.9 & 0.891 & & 4.55 & 0.891 & & 177 & 118 & $\mathrm{U}$ \\
\hline & 774.2 & 1.46 & 0.708 & UJ & 3.91 & 0.962 & & 186 & 118 & $\mathrm{U}$ \\
\hline & 836 & 2.78 & 0.948 & $\mathrm{~J}$ & 3.65 & 0.878 & & 273 & 123 & UJ \\
\hline \multicolumn{11}{|c|}{$\begin{array}{l}\text { a. The MCL for gross beta is based on an exposure criterion of } 4 \mathrm{mrem} / \text { year. Gross beta concentrations are not directly compared to the MCL } \\
\text { because the units are not the same. Gross beta results are used as an indicator of contamination. } \\
\text { b. The uncertainty value is one standard deviation. } \\
\text { c. See Appendix A, Section A-2, for data flag definitions. } \\
\text { d. The data are reported as received from the laboratory. Negative analytical results for radiological analytes may occur as a result of counting } \\
\text { procedures and result of calculation. }\end{array}$} \\
\hline $\begin{array}{l}\text { Dup } \\
\text { INL } \\
\text { MCL } \\
\text { NS }\end{array}$ & $\begin{array}{l}\text { duplica } \\
\text { Idaho } ~ \\
\text { maxim } \\
\text { not san }\end{array}$ & $\begin{array}{l}\text { ory } \\
\text { level }\end{array}$ & & & & & & & & \\
\hline
\end{tabular}

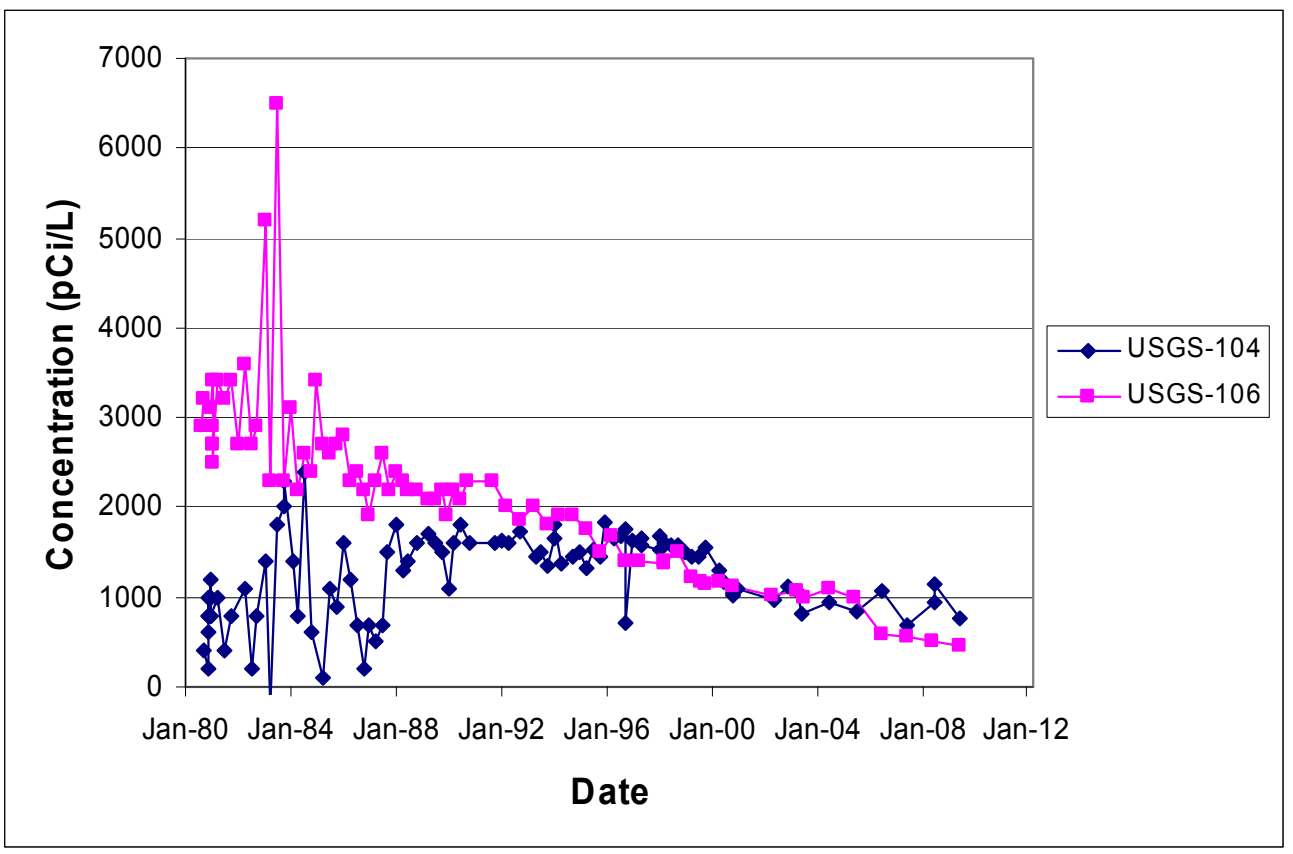

Figure 5. Tritium concentration trends for Wells USGS-104 and -106. 


\subsubsection{Volatile Organic Compounds}

Two volatile organic compounds - toluene, and carbon tetrachloride - were detected at concentrations well below MCLs. Toluene was detected in the sample from the Highway 3 well at a concentration of $0.56 \mu \mathrm{g} / \mathrm{L}$. The toluene detection was well below the MCL for toluene (i.e., 1,000 $\mu \mathrm{g} / \mathrm{L}$ ) and near the practical quantitation limit (i.e., $1 \mu \mathrm{g} / \mathrm{L}$ ). The source of toluene in this well is uncertain. Toluene could be a laboratory contaminant; therefore, that source for the toluene cannot be ruled out. Vehicle exhaust is another possible explanation for the toluene detection because the well is located within a highway rest stop area. Carbon tetrachloride was detected in Well USGS-109, located south of RWMC on the INL Site boundary, and was also detected in one interval from Westbay Well USGS-132, located south of RWMC. The maximum detected concentration was $0.452 \mu \mathrm{g} / \mathrm{L}$ in the uppermost sample (i.e., $646.7 \mathrm{ft}$ bgs) from Well USGS-132 south of RWMC. Detected carbon tetrachloride values are estimated or J-flagged and are close to the method detection limit. The detected carbon tetrachloride concentrations are below the MCL of $5 \mu \mathrm{g} / \mathrm{L}$. A carbon tetrachloride plume originates at RWMC, and carbon tetrachloride detections probably represent migration from RWMC.

\subsubsection{Metals and Anions}

Table 4 provides summary data for anions, common cations, and metals and compares them to MCLs or secondary MCLs. Data also are compared to USGS background values for the INL Site (USGS 1999). When data for WAG 10 boundary and guard wells are compared to MCLs and secondary MCLs, iron is the only analyte above its MCL, secondary MCL, or U.S. Environmental Protection Agency action level. Iron was detected above its secondary MCL in one well, USGS-110. However, reported iron concentrations for several wells are inconsistent with observed dissolved-oxygen levels and slightly alkaline $\mathrm{pH}$ in the aquifer because iron has a very low solubility in these conditions (Figure 6). Iron concentrations in several wells have increased in recent years, but the cause of the elevated iron concentrations is uncertain. Lead occurred at concentrations of 14.1 and $13.3 \mu \mathrm{g} / \mathrm{L}$ in Wells USGS-100 and -106, respectively. These concentrations are close to the action level of $15 \mu \mathrm{g} / \mathrm{L}$ for lead. Both occurrences were in wells with above-background zinc concentration. The association of elevated lead and zinc concentrations at Wells USGS-100 and USGS-106 is probably due to corrosion of galvanized riser pipe, as discussed below.

Though not above its secondary MCL, zinc concentrations in the groundwater samples from Wells USGS-100 and USGS-106 are well above background concentrations (Figure 7). Elevated zinc concentrations in these groundwater monitoring wells are probably the result of corroding galvanized discharge or riser pipe used in their construction. Elevated zinc concentrations in groundwater have been correlated to galvanized riser pipes for other wells at the INL Site (INEEL 2003; ICP 2004). After the galvanized riser pipes in Wells USGS-86, USGS-104, and USGS-109 were replaced, zinc concentrations in those wells dropped to background levels (Figure 6). Zinc concentrations in USGS-103 have decreased in all Westbay intervals compared to zinc results before replacing the riser pipe and pump with the Westbay system.

Though not above its MCL, nitrate concentrations in Wells USGS-100 and -002-south and southwest of the Materials and Fuels Complex - have been increasing (Figure 8). In addition, nitrate concentrations in Well USGS-101 also show an increasing trend, but to a lesser extent than for Wells USGS-100 and USGS-002. Increasing nitrate concentrations can be an indicator of contamination. The spike in nitrate concentrations observed in FY 2007, at Wells USGS-100 and -002, has reverted to the long-term, slow-increase trend. The source of slowly increasing nitrate concentrations is probably migration of agricultural contamination from the northeastern boundary of the INL Site, across the eastern part of the INL Site (DOE-ID 2008a), rather than contamination from INL Site facilities. Migration of agricultural contaminants along the eastern side of the INL Site is consistent with groundwater flow paths indicated by INL Site-wide water-level measurements and the Site-wide automated monitoring network (DOE-ID 2005, 2006, 2008a). 
Table 4. Summary of inorganic results.

\begin{tabular}{|c|c|c|c|c|c|c|c|c|c|}
\hline \multirow[b]{2}{*}{ Compound } & \multirow{2}{*}{$\begin{array}{c}\text { Sample } \\
\text { Units }\end{array}$} & \multicolumn{2}{|c|}{ Guard Well $^{\mathrm{a}}$} & \multicolumn{2}{|c|}{ Boundary Well $^{\mathrm{b}}$} & \multirow{2}{*}{$\begin{array}{c}\text { Maximum } \\
\text { Contaminant } \\
\text { Level or Secondary } \\
\text { Maximum } \\
\text { Contaminant Level }^{\mathrm{c}}\end{array}$} & \multirow[b]{2}{*}{ Background $^{\mathrm{d}}$} & \multirow{2}{*}{$\begin{array}{l}\text { Detections } \\
\text { Above } \\
\text { Background }\end{array}$} & \multirow{2}{*}{$\begin{array}{l}\text { Detections Above } \\
\text { Maximum Contaminant Leve } \\
\text { or Secondary Maximum } \\
\text { Contaminant Level }\end{array}$} \\
\hline & & Min. & Max. & Min. & Max. & & & & \\
\hline \multicolumn{10}{|l|}{ Anions } \\
\hline Alkalinity & $\mathrm{mg} / \mathrm{L}$ & 121 & 155 & 104 & 145 & None & $169-174$ & No & NA \\
\hline Chloride & $\mathrm{mg} / \mathrm{L}$ & 5.55 & 21.2 & 9.11 & 19.2 & 250 & $16-27$ & No & 0 \\
\hline Sulfate & $\mathrm{mg} / \mathrm{L}$ & 15.2 & 37.2 & 10.3 & 26.1 & 250 & $24-31$ & Yes & 0 \\
\hline \multicolumn{10}{|c|}{ Common Cations } \\
\hline Calcium & $\mu \mathrm{g} / \mathrm{L}$ & 33,200 & 43,300 & 22,100 & 41,200 & None & $43,000-46,000$ & No & NA \\
\hline Magnesium & $\mu \mathrm{g} / \mathrm{L}$ & 10,400 & 17,500 & 8,930 & 18,100 & None & 15,000 & Yes & NA \\
\hline Potassium & $\mu \mathrm{g} / \mathrm{L}$ & 2,000 & 3,510 & 2,260 & 2,990 & None & $3,100-3,500$ & Yes & NA \\
\hline Sodium & $\mu \mathrm{g} / \mathrm{L}$ & 4,950 & 24,700 & 9,600 & 16,100 & None & $14,000-17,000$ & Yes & NA \\
\hline Arsenic & $\mu \mathrm{g} / \mathrm{L}$ & 1.6 & 4.82 & 1.6 & 5.37 & 10 & $2-3$ & Yes & 0 \\
\hline Barium & $\mu \mathrm{g} / \mathrm{L}$ & 31 & 56 & 17.4 & 45.8 & 2,000 & $50-70$ & No & 0 \\
\hline Beryllium & $\mu \mathrm{g} / \mathrm{L}$ & $\mathrm{U}$ & $\mathrm{U}$ & $\mathrm{U}$ & $\mathrm{U}$ & 4 & - & - & 0 \\
\hline Cadmium & $\mu \mathrm{g} / \mathrm{L}$ & $\mathrm{U}$ & 0.525 & $\mathrm{U}$ & $\mathrm{U}$ & 5 & $<1$ & No & 0 \\
\hline Chromium & $\mu \mathrm{g} / \mathrm{L}$ & $\mathrm{U}$ & 9.91 & $\mathrm{U}$ & 11.5 & 100 & $2-3$ & Yes & 0 \\
\hline Cobalt & $\mu \mathrm{g} / \mathrm{L}$ & $\mathrm{U}$ & 4.33 & $\mathrm{U}$ & 3.78 & None & 3 & Yes & NA \\
\hline Copper & $\mu \mathrm{g} / \mathrm{L}$ & 0.349 & 1.24 & 0.455 & 0.982 & $1,300 / 1,000$ & $<1$ & Yes & 0 \\
\hline Iron & $\mu \mathrm{g} / \mathrm{L}$ & 77.9 & 237 & 65.7 & 361 & 300 & $16-25$ & Yes & 1 \\
\hline Lead & $\mu \mathrm{g} / \mathrm{L}$ & $\mathrm{U}$ & 14.1 & $\mathrm{U}$ & $\mathrm{U}$ & $15^{\mathrm{e}}$ & $1-5$ & Yes & 0 \\
\hline Manganese & $\mu \mathrm{g} / \mathrm{L}$ & $\mathrm{U}$ & 7.12 & $\mathrm{U}$ & 48.5 & 50 & 7 & Yes & 0 \\
\hline Mercury & $\mu \mathrm{g} / \mathrm{L}$ & $\mathrm{U}$ & $\mathrm{U}$ & $\mathrm{U}$ & $\mathrm{U}$ & 2 & - & No & 0 \\
\hline Nickel & $\mu \mathrm{g} / \mathrm{L}$ & 0.756 & 1.43 & 0.671 & 1.82 & None & 10 & No & NA \\
\hline
\end{tabular}


Table 4. (continued).

\begin{tabular}{|c|c|c|c|c|c|c|c|c|c|}
\hline \multirow[b]{2}{*}{ Compound } & \multirow[b]{2}{*}{$\begin{array}{c}\text { Sample } \\
\text { Units }\end{array}$} & \multicolumn{2}{|c|}{ Guard Well ${ }^{\mathrm{a}}$} & \multicolumn{2}{|c|}{ Boundary Well $^{\mathrm{b}}$} & \multirow{2}{*}{$\begin{array}{c}\text { Maximum } \\
\text { Contaminant } \\
\text { Level or Secondary } \\
\text { Maximum } \\
\text { Contaminant Level }^{\mathrm{c}} \\
\end{array}$} & \multirow[b]{2}{*}{ Background $^{\mathrm{d}}$} & \multirow{2}{*}{$\begin{array}{l}\text { Detections } \\
\text { Above } \\
\text { Background }\end{array}$} & \multirow{2}{*}{$\begin{array}{c}\text { Detections Above } \\
\text { Maximum Contaminant Level } \\
\text { or Secondary Maximum } \\
\text { Contaminant Level }\end{array}$} \\
\hline & & Min. & Max. & Min. & Max. & & & & \\
\hline Strontium & $\mu \mathrm{g} / \mathrm{L}$ & 139 & 236 & 91.9 & 220 & None & $220-237$ & No & NA \\
\hline Thallium & $\mu \mathrm{g} / \mathrm{L}$ & $\mathrm{U}$ & 0.373 & $\mathrm{U}$ & 0.375 & 2 & - & - & 0 \\
\hline Zinc & $\mu \mathrm{g} / \mathrm{L}$ & $\mathrm{U}$ & 189 & $\mathrm{U}$ & 45.2 & 5,000 & $10.5-54$ & Yes & 0 \\
\hline
\end{tabular}

a. Guard wells include sampling from Westbay Well USGS-132.

b. Boundary wells include sampling of Westbay Well USGS-103.

c. Numbers in italic are for secondary maximum contaminant levels.

d. Background is from two sources. Plain numbers are from Knobel, Orr, and Cecil (1992); italicized numbers are from USGS (1999)—median and mean values. Note that these two sources are Site-wide background values; local background may be different.

e. The action level for lead is $15 \mu \mathrm{g} / \mathrm{L}$.

$$
\begin{aligned}
& \text { NA no background level was determined } \\
& \text { nA applicable }
\end{aligned}
$$

$\mathrm{U}$ data qualifier flag: the analyte was analyzed for but not detected 


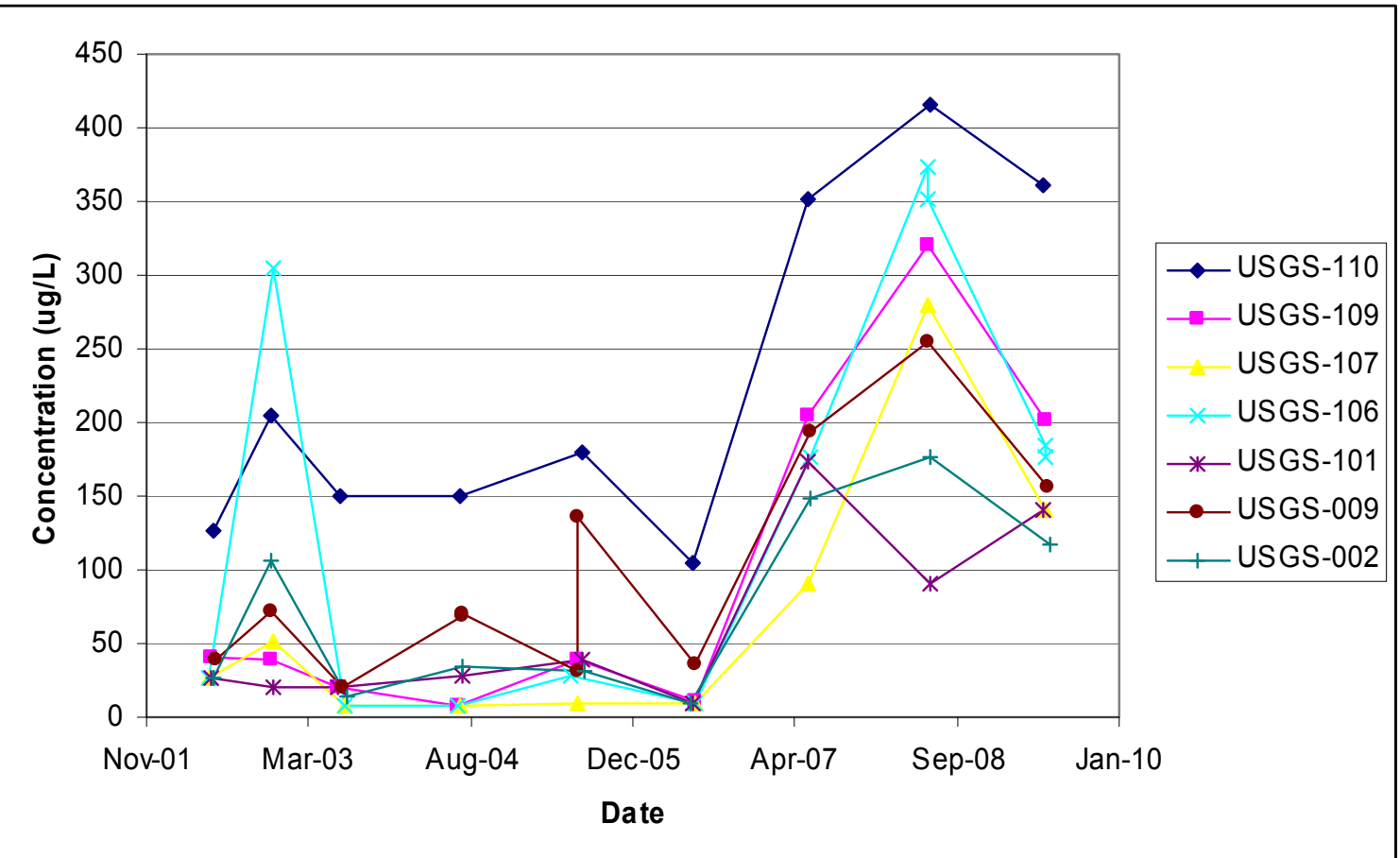

Figure 6. Iron concentrations in select wells, November 2001 through January 2010.

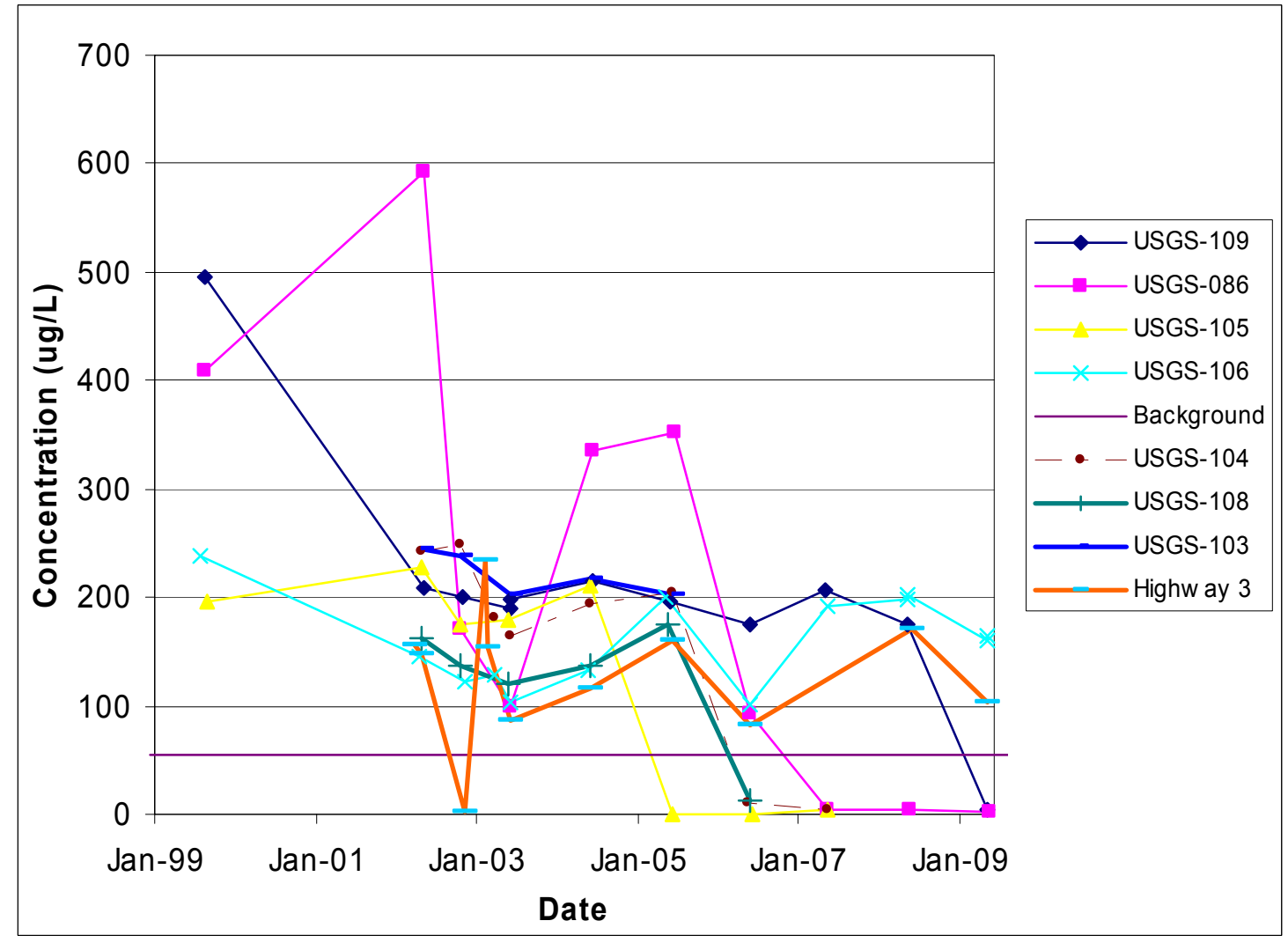

Figure 7. Zinc concentrations at selected wells that contain galvanized riser pipes, January 1999 through January 2009. 


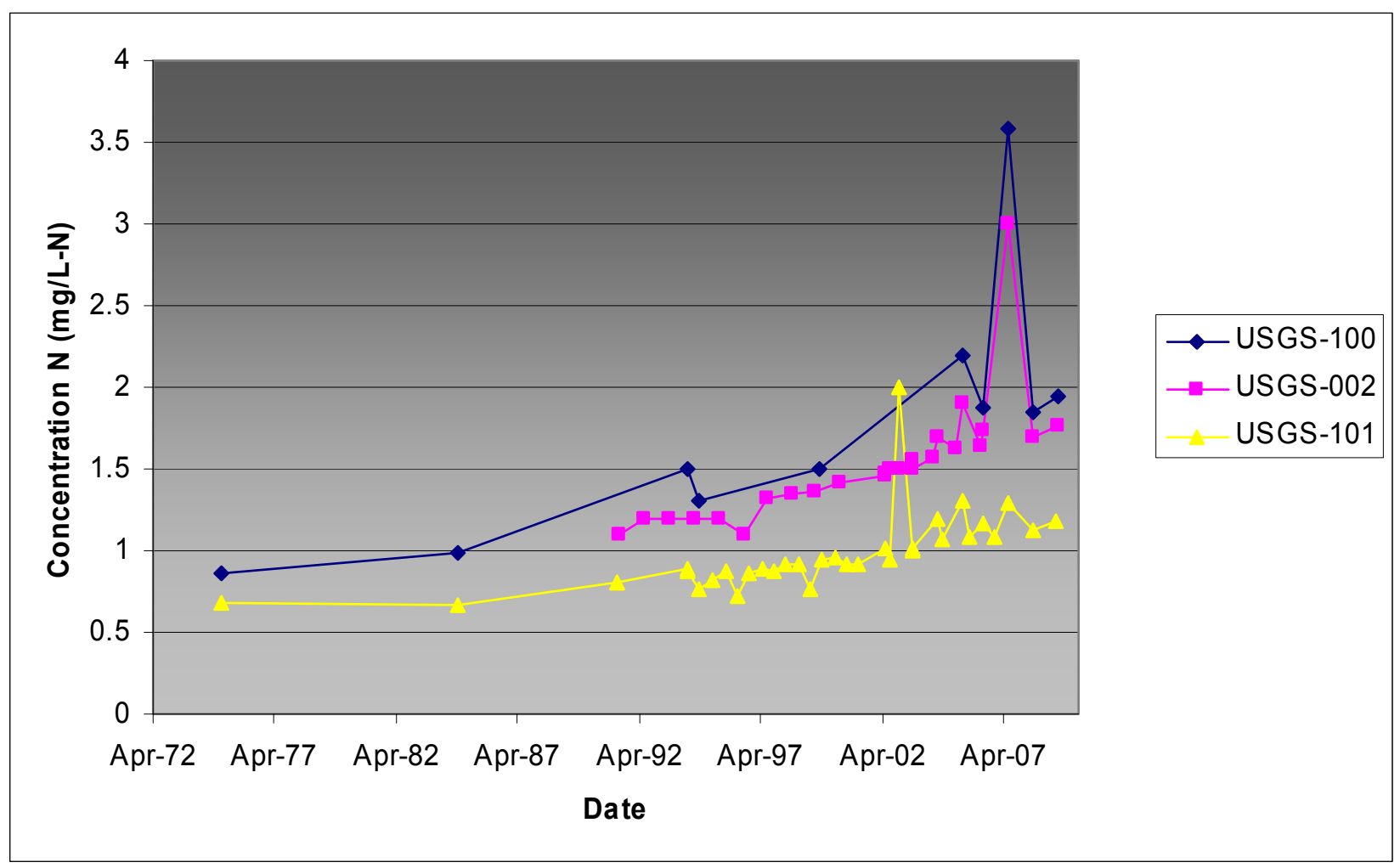

Figure 8. Nitrate trends in Wells USGS-100, -002, and -101, April 1972 through April 2007.

\subsection{Vertical Concentration Profiles for Westbay Wells}

Westbay multilevel sampling systems were installed in Wells USGS-103 and -132 to evaluate the vertical distribution of contaminants. Well USGS-132 is located south of RWMC, and Well USGS-103 is located south of the Central Facilities Area on the southern boundary of the INL Site. Vertical distribution of analytes will be used to select possible sampling intervals for long-term monitoring.

Figure 9 shows vertical distributions of tritium, chloride, and sulfate at Well USGS-132, south of RWMC, for the three intervals sampled. Chloride and sulfate concentrations show a sharp decrease from the 646-ft sampling depth to the 774-ft sampling depth. In addition to elevated anions, carbon tetrachloride was detected in the 646-ft-depth sample. The occurrence of elevated anions and carbon tetrachloride indicates that this interval is monitoring migration from the RWMC area. This chloride and sulfate distribution is consistent with past sampling. Higher chloride and sulfate concentrations in the 646-ft-depth sample are similar to concentrations from wells that sample near the water table south of RWMC (DOE-ID 2008a, 2008b). Tritium was below the minimum detectable activity at all depths.

Figure 10 shows vertical distributions of tritium, chloride, and sulfate at Well USGS-103, which is on the southern boundary of the INL Site. Chloride concentrations show a sharp decrease from the 681.9-ft sampling depth to the 913.8-ft sampling depth. In contrast to chloride, sulfate concentrations increased slightly from the 681.9-ft depth to the 805.2-ft depth; whereas, previously, chloride and sulfate both declined. The cause of the increase in sulfate concentrations from the 681.9- $\mathrm{ft}$ depth to the $805.2-\mathrm{ft}$ depth is uncertain. From the 913.8-ft depth to the deepest port (i.e., 1,269 ft bgs), sulfate and chloride concentrations show a slight increase. Tritium data for Well USGS-103 show detections near the minimum detectable activity at 1,095.1 and 1,269.4 ft bgs. In 2008, tritium was below the MDA in all 


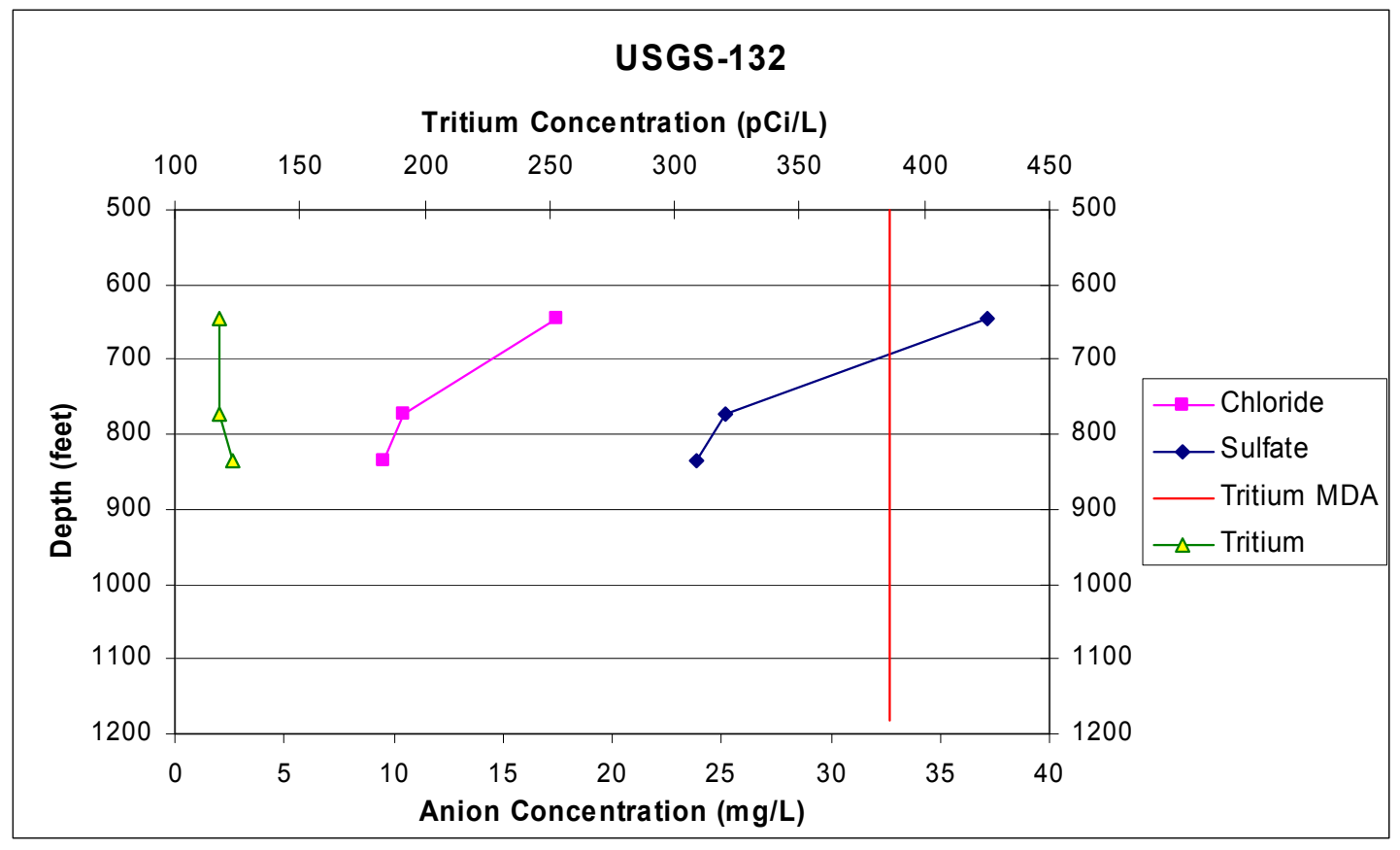

Figure 9. Vertical distribution of tritium, chloride, and sulfate in Well USGS-132.

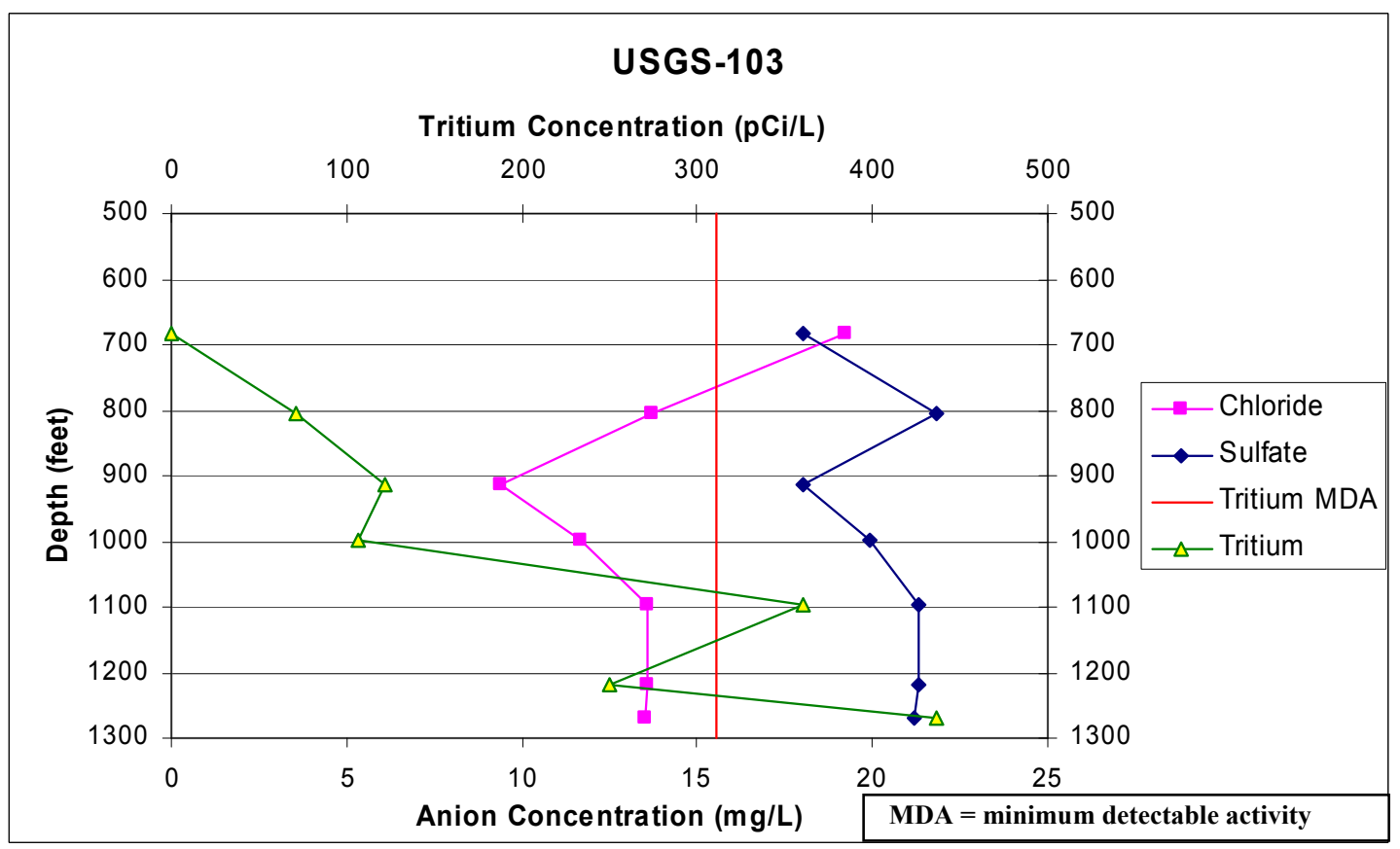

Figure 10. Vertical distribution of tritium, chloride, and sulfate in Well USGS-103. 
intervals except 999 and 1,095 ft bgs; however, the result for the deepest sample (1,269 ft bgs) was flagged as non-detect because of blank contamination. The 2009 result for the 1,269-ft-depth sample yielded a value similar to the 2008 sample; however, in contrast, the 2008 result was not flagged non-detect because of blank contamination. The other difference between the 2008 and 2009 data is that tritium was detected in the 999-ft-depth sample in 2008 but not in the 2009 sample from the same depth. Because tritium measured only marginally above the MDA in 2008, the non-detect from 999-ft-depth sample in 2009 is only marginally different.

\section{SUMMARY}

Groundwater samples collected from 12 wells and 2 Westbay wells, with 10 sampling intervals, were sampled for OU 10-08 monitoring. During FY 2009 groundwater monitoring, no contaminant exceeded MCLs in any wells along the southern boundary of the INL Site or in the guard wells. Lead was detected just below its action level of $15 \mu \mathrm{g} / \mathrm{L}$ in Wells USGS-100 and USGS-106. However, the elevated lead concentrations in Wells USGS-100 and -106 are probably the result of the corrosion of galvanized riser pipes. Elevated zinc concentrations in these wells implicate the galvanized riser pipe as the cause of the elevated lead concentration. Once the galvanized pipe is replaced, both lead and zinc concentrations should decline as has been observed at other WAG 10 wells.

Iron concentration in Well USGS-110 was $361 \mu \mathrm{g} / \mathrm{L}$, which is higher than its secondary MCL of $300 \mu \mathrm{g} / \mathrm{L}$. However, the elevated iron concentration is not consistent with the observed high dissolved-oxygen concentrations and the slightly alkaline $\mathrm{pH}$ of the aquifer. Dissolved iron concentrations in the aquifer should be low due to the oxidizing conditions and slightly alkaline $\mathrm{pH}$. The cause of the high iron concentrations is uncertain because the iron and dissolved oxygen data are inconsistent.

\section{REFERENCES}

42 USC § 9601 et seq., 1980, "Comprehensive Environmental Response, Compensation, and Liability Act of 1980 (CERCLA/Superfund)," United States Code, December 11, 1980.

DOE-ID, 1991, Federal Facility Agreement and Consent Order for the Idaho National Engineering Laboratory, Administrative Docket No. 1088-06-29-120, U.S. Department of Energy Idaho Operations Office; U.S. Environmental Protection Agency, Region 10; Idaho Department of Health and Welfare, December 4, 1991.

DOE-ID, 2002, Waste Area Group 10, Operable Unit 10-08, Remedial Investigation/Feasibility Study Work Plan (FINAL), DOE/ID-10902, Rev. 0, U.S. Department of Energy Idaho Operations Office, August 2002.

DOE-ID, 2005, Waste Area Group 10, Operable Unit 10-08, Remedial Investigation/Feasibility Study Annual Status Report for Fiscal Year 2004, DOE/NE-ID-11198, Rev. 0, U.S. Department of Energy Idaho Operations Office, March 2005.

DOE-ID, 2006, Waste Area Group 10, Operable Unit 10-08, Remedial Investigation/Feasibility Study Annual Status Report for Fiscal Year 2005, DOE/ID-11274, Rev. 1, U.S. Department of Energy Idaho Operations Office, May 2006.

DOE-ID, 2007a, Groundwater Monitoring and Field Sampling Plan for Operable Unit 10-08, DOE/NE-ID-11210, Rev. 2, U.S. Department of Energy Idaho Operations Office, May 2007. 
DOE-ID, 2007b, Waste Area Group 10, Operable Unit 10-08, Remedial Investigation/Feasibility Study Annual Status Report for Fiscal Year 2006, DOE/ID-11297, Rev. 0, U.S. Department of Energy Idaho Operations Office, May 2007.

DOE-ID, 2008a, Waste Area Group 10, Operable Unit 10-08, Annual Monitoring Status Report for Fiscal Year 2007, DOE/ID-11355, Rev. 0, U.S. Department of Energy Idaho Operations Office, March 2008.

DOE-ID, 2008b, Operable Unit 10-08 Sitewide Groundwater and Miscellaneous Sites Remedial Investigation/Baseline Risk Assessment, DOE/ID-11332, Rev. 0, U.S. Department of Energy Idaho Operations Office, April 2008.

DOE-ID, 2009a, Operable Unit 10-08 Record of Decision for Site-Wide Groundwater, Miscellaneous Sites, and Future Sites, DOE/ID-11385, Rev. 0, U.S. Department of Energy Idaho Operations Office, September 2009.

DOE-ID, 2009b, Waste Area Group 10, Operable Unit 10-08, Annual Monitoring Status Report for Fiscal Year 2008, DOE/ID-11388, Rev. 0, U.S. Department of Energy Idaho Operations Office, April 2009.

ICP, 2004, Annual Groundwater Monitoring Status Report for Waste Area Group 5 for Fiscal Year 2004, ICP/EXT-04-00369, Rev. 1, Idaho Completion Project, Idaho National Engineering and Environmental Laboratory, November 2004.

INEEL, 2003, Central Facilities Area Landfills I, II, and III Annual Monitoring Report (2002), INEEL/EXT-03-00024, Rev. 0, Idaho National Engineering and Environmental Laboratory, September 2003.

Knobel, L. L., B. R. Orr, and L. D. Cecil, 1992, "Summary of Concentrations of Selected Radiochemical and Chemical Constituents in Groundwater from the Snake River Plain Aquifer, Idaho: Estimated from an Analysis of Previously Published Data," Journal of Idaho Academy of Science, Vol. 28, No. 1, pp. 48-61, June 1992.

Public Law 99-499, 1986, "Superfund Amendments and Reauthorization Act of 1986 (SARA)," 100 Statutes 1728, Public Law, October 17, 1986.

USGS, 1999, Chemical Constituents in Ground Water from 39 Selected Sites with an Evaluation of Associated Quality Assurance Data, Idaho National Engineering and Environmental Laboratory and Vicinity, Idaho, USGS Open File Report 99-246, DOE/ID-22159, United States Geological Survey, August 1999. 


\section{Appendix A}

\section{Analytical Results from Fiscal Year 2009 Groundwater Sampling}




$$
\text { A-2 }
$$




\section{Appendix A}

\section{Analytical Results from Fiscal Year 2009 Groundwater Sampling for Waste Area Group 10 \\ A-1. INTRODUCTION}

This appendix presents groundwater analytical results from Fiscal Year (FY) 2009 for Waste Area Group (WAG) 10 at the Idaho National Laboratory Site. Table A-1 presents a summary of the analytes and analytical methods.

Table A-1. Analytes and method codes.

\begin{tabular}{lll}
\hline \multicolumn{1}{c}{ Analyte } & Method Code & \multicolumn{1}{c}{ Method Description } \\
\hline $\begin{array}{l}\text { Volatile organic compounds } \\
\begin{array}{l}\text { Sulfate, chloride, fluoride, } \\
\text { nitrogen, nitrate/nitrite }\end{array}\end{array}$ & SW8260B & $\begin{array}{l}\text { Volatile organic compounds by gas } \\
\text { chromatography/mass spectrometry } \\
\text { Alkalinity, total as CaCO } 3\end{array}$ \\
$\begin{array}{l}\text { Tritium } \\
\text { Metals, except mercury }\end{array}$ & SM2320B-B & Bicarbonate by Standard Method 2320B \\
Mercury & SW6020 & Liquid scintillation \\
Sr-90 & SW7470A & $\begin{array}{l}\text { Mercury in liquid waste (manual cold-vapor } \\
\text { technique) }\end{array}$ \\
Tc-99 & GFP & Gas flow proportional counter \\
I-129 & LSC & Liquid scintillation \\
Gross alpha & OTR & Other radiochemical analyses \\
Gross beta & GAB & Gross alpha/beta \\
& GAB & Gross alpha/beta \\
\hline
\end{tabular}




\section{A-2. DATA QUALIFIER FLAGS}

Data qualifier flags used in this appendix comprise a consolidation of laboratory- and validation-assigned flags. Data qualifier flags for organic, inorganic, and radiological results are defined as follows:

\section{Organic Qualifier Flags:}

- $\quad$ B-Analyte was detected in the associated laboratory method blank as well as in the sample.

- $\quad$ U-Analyte was analyzed for but not detected.

- $\quad$ UJ-Associated value is an estimate and might be inaccurate or imprecise. The result is considered a nondetect for project data interpretation purposes.

- $\quad \mathrm{J}$-Analyte was detected, but the associated values are an estimate and might be inaccurate or imprecise.

- $\quad \mathrm{N}$-Presumptive evidence indicates that a compound is present.

- $\quad \mathrm{NJ}$ or JN-Presumptive evidence indicates that a compound is present, and associated values are an estimate.

- $\quad \mathrm{R}$-Accuracy of the data is so questionable that it is recommended that the data not be used. The " $R$ " flag overrides all other applicable flags.

\section{Inorganic Qualifier Flags:}

- $\quad \mathrm{B}$ - The result is less than the contract-required reporting limit but greater than or equal to the instrument detection limit.

- $\quad$ E-Reported value was estimated because of the presence of interference.

- $\quad \mathrm{N}$-The spiked sample recovery was outside control limits.

- $\quad \mathrm{U}-$ The analyte was not detected.

- $\quad$ UJ-The associated value is an estimate and might be inaccurate or imprecise. The result is considered a nondetect for project data-interpretation purposes.

- $\quad \mathrm{R}$-Accuracy of the data is so questionable that it is recommended that the data not be used. The " $R$ " flag overrides all other applicable flags.

\section{Radiological Qualifier Flags:}

- $\quad \mathrm{J}-$ The associated value is estimated. The result might not be an accurate representation of the amount of activity present in the sample.

- $\quad \mathrm{R}$-Accuracy of the data is so questionable that it is recommended that the data not be used. The " $R$ " flag overrides all other applicable flags. 
- $\quad \mathrm{U}$-The radionuclide is not considered present in the sample (i.e., nondetect).

- $\quad$ UJ-The radionuclide might or might not be present, and the result is considered highly questionable. The associated value is an estimate and might be inaccurate or imprecise. The result is considered a nondetect for project data interpretation purposes.

\section{A-3. GROUNDWATER DATA}

Table A-2 presents FY 2009 monitoring data for WAG 10 (i.e., boundary, guard, and Westbay wells). The complete groundwater data set (in Excel) is provided on the compact disc attached to the inside back cover of this report.

Note that alkalinity data are reported in units of $\mathrm{mg} / \mathrm{L}$ as calcium carbonate. In Table A-2 and on the compact disc, samples and duplicate samples are designated as 01 and 02 before the two-character analysis code. For example, sample GWA047022X is the duplicate metals sample for GWA047012X from Well USGS-104. The analytical result and a "U" flag is the practical quantitation limit for the nonradiological analytes. 
Table A-2. Waste Area Group 10 groundwater monitoring data for Fiscal Year 2009.

\begin{tabular}{|c|c|c|c|c|c|c|c|c|c|c|c|}
\hline $\begin{array}{c}\text { Field Sample } \\
\text { Number }\end{array}$ & Location & Depth & Compound & $\begin{array}{l}\text { Sample } \\
\text { Result }\end{array}$ & $\begin{array}{c}\text { Sample } \\
\text { Error }\end{array}$ & $\begin{array}{c}\text { Result } \\
\text { Qualifier }\end{array}$ & $\begin{array}{c}\text { Validation } \\
\text { Flag }\end{array}$ & $\begin{array}{c}\text { Sample } \\
\text { Units }\end{array}$ & $\begin{array}{c}\text { Date Sample } \\
\text { Collected }\end{array}$ & MDA & L\&V Report Number \\
\hline GWA044012X & HIGHWAY 3 & 538 & Aluminum & 15 & & $U$ & & UG/L & $06 / 09 / 2009$ & & HCJ-161-09 \\
\hline GWA044012X & HIGHWAY 3 & 538 & Antimony & 1 & & U & & UG/L & 06/09/2009 & & HCJ-161-09 \\
\hline GWA044012X & HIGHWAY 3 & 538 & Arsenic & 1.6 & & U & & UG/L & $06 / 09 / 2009$ & & HCJ-161-09 \\
\hline GWA044012X & HIGHWAY 3 & 538 & Barium & 50.2 & & B & & UG/L & 06/09/2009 & & HCJ-161-09 \\
\hline GWA044012X & HIGHWAY 3 & 538 & Beryllium & 0.1 & & U & & UG/L & 06/09/2009 & & HCJ-161-09 \\
\hline GWA044012X & HIGHWAY 3 & 538 & Cadmium & 0.11 & & $U$ & & UG/L & 06/09/2009 & & HCJ-161-09 \\
\hline GWA044012X & HIGHWAY 3 & 538 & Calcium & 40600 & & & & UG/L & 06/09/2009 & & HCJ-161-09 \\
\hline GWA044012X & HIGHWAY 3 & 538 & Chromium & 2 & & U & & UG/L & 06/09/2009 & & HCJ-161-09 \\
\hline GWA044012X & HIGHWAY 3 & 538 & Cobalt & 1.17 & & B & & UG/L & 06/09/2009 & & HCJ-161-09 \\
\hline GWA044012X & HIGHWAY 3 & 538 & Copper & 0.862 & & B & & UG/L & $06 / 09 / 2009$ & & HCJ-161-09 \\
\hline GWA044012X & HIGHWAY 3 & 538 & Iron & 173 & & & & UG/L & $06 / 09 / 2009$ & & HCJ-161-09 \\
\hline GWA044012X & HIGHWAY 3 & 538 & Lead & 0.5 & & U & & UG/L & 06/09/2009 & & HCJ-161-09 \\
\hline GWA044012X & HIGHWAY 3 & 538 & Magnesium & 10900 & & & & UG/L & $06 / 09 / 2009$ & & HCJ-161-09 \\
\hline GWA044012X & HIGHWAY 3 & 538 & Manganese & 3.05 & & B & & UG/L & $06 / 09 / 2009$ & & HCJ-161-09 \\
\hline GWA044012X & HIGHWAY 3 & 538 & Mercury & 0.067 & & U & & UG/L & 06/09/2009 & & HCJ-161-09 \\
\hline GWA044012X & HIGHWAY 3 & 538 & Nickel & 0.89 & & B & & UG/L & 06/09/2009 & & HCJ-161-09 \\
\hline GWA044012X & HIGHWAY 3 & 538 & Potassium & 2150 & & B & & UG/L & $06 / 09 / 2009$ & & HCJ-161-09 \\
\hline GWA044012X & HIGHWAY 3 & 538 & Selenium & 1 & & $u$ & & UG/L & $06 / 09 / 2009$ & & HCJ-161-09 \\
\hline GWA044012X & HIGHWAY 3 & 538 & Silver & 0.2 & & U & & UG/L & 06/09/2009 & & HCJ-161-09 \\
\hline GWA044012X & HIGHWAY 3 & 538 & Sodium & 4950 & & B & & UG/L & 06/09/2009 & & HCJ-161-09 \\
\hline GWA044012X & HIGHWAY 3 & 538 & Strontium & 236 & & & & UG/L & $06 / 09 / 2009$ & & HCJ-161-09 \\
\hline GWA044012X & HIGHWAY 3 & 538 & Thallium & 0.3 & & $\mathrm{U}$ & & UG/L & 06/09/2009 & & HCJ-161-09 \\
\hline GWA044012X & HIGHWAY 3 & 538 & Uranium & 2.14 & & B & & UG/L & $06 / 09 / 2009$ & & HCJ-161-09 \\
\hline GWA044012X & HIGHWAY 3 & 538 & Vanadium & 4.25 & & B & & UG/L & $06 / 09 / 2009$ & & HCJ-161-09 \\
\hline GWA044012X & HIGHWAY 3 & 538 & Zinc & 103 & & & & UG/L & 06/09/2009 & & HCJ-161-09 \\
\hline GWA04401A1 & HIGHWAY 3 & 538 & Alkalinity, Total as $\mathrm{CaCO} 3$ & 146 & & & & $M G / L$ & 06/09/2009 & & HCJ-157-09 \\
\hline GWA04401AN & HIGHWAY 3 & 538 & Bromide & 0 & & U & & $M G / L$ & $06 / 09 / 2009$ & & HCJ-159-09 \\
\hline GWA04401AN & HIGHWAY 3 & 538 & Chloride & 5.55 & & & & $M G / L$ & $06 / 09 / 2009$ & & HCJ-159-09 \\
\hline GWA04401AN & HIGHWAY 3 & 538 & Fluoride & 0.237 & & $\mathrm{~J}$ & & $M G / L$ & 06/09/2009 & & HCJ-159-09 \\
\hline GWA04401AN & HIGHWAY 3 & 538 & Sulfate & 20.1 & & & & $M G / L$ & $06 / 09 / 2009$ & & HCJ-159-09 \\
\hline GWA04401N2 & HIGHWAY 3 & 538 & Nitrogen, Nitrate/Nitrite & 0.5 & & & & $M G / L$ & $06 / 09 / 2009$ & & HCJ-155-09 \\
\hline GWA04401VL & HIGHWAY 3 & 538 & Dichlorodifluoromethane & 1 & & $\mathrm{U}$ & & UG/L & 06/09/2009 & & HCJ-148-09 \\
\hline GWA04401VL & HIGHWAY 3 & 538 & Chloromethane & 1 & & $\mathrm{U}$ & & UG/L & 06/09/2009 & & HCJ-148-09 \\
\hline GWA04401VL & HIGHWAY 3 & 538 & Vinyl Chloride & 1 & & $\mathrm{U}$ & & UG/L & $06 / 09 / 2009$ & & HCJ-148-09 \\
\hline GWA04401VL & HIGHWAY 3 & 538 & Bromomethane & 1 & & $u$ & & UG/L & 06/09/2009 & & HCJ-148-09 \\
\hline GWA04401VL & HIGHWAY 3 & 538 & Chloroethane & 1 & & $\mathrm{U}$ & & UG/L & 06/09/2009 & & HCJ-148-09 \\
\hline GWA04401VL & HIGHWAY 3 & 538 & Trichlorofluoromethane & 1 & & $\mathrm{u}$ & & UG/L & 06/09/2009 & & HCJ-148-09 \\
\hline GWA04401VL & HIGHWAY 3 & 538 & Acetone & 5 & & $U$ & & UG/L & 06/09/2009 & & HCJ-148-09 \\
\hline GWA04401VL & HIGHWAY 3 & 538 & 1,1-Dichloroethene & 1 & & $\mathrm{U}$ & & UG/L & 06/09/2009 & & HCJ-148-09 \\
\hline GWA04401VL & HIGHWAY 3 & 538 & Methyl acetate & 5 & & $\mathrm{u}$ & & UG/L & 06/09/2009 & & HCJ-148-09 \\
\hline GWA04401VL & HIGHWAY 3 & 538 & Methylene Chloride & 5 & & $\mathrm{U}$ & & UG/L & 06/09/2009 & & HCJ-148-09 \\
\hline GWA04401VL & HIGHWAY 3 & 538 & Carbon disulfide & 5 & & $u$ & & UG/L & 06/09/2009 & & HCJ-148-09 \\
\hline GWA04401VL & HIGHWAY 3 & 538 & Tert-butyl methyl ether & 1 & & $\mathrm{U}$ & & UG/L & 06/09/2009 & & HCJ-148-09 \\
\hline GWA04401VL & HIGHWAY 3 & 538 & trans-1,2-Dichloroethylene & 1 & & $\mathrm{u}$ & & UG/L & 06/09/2009 & & HCJ-148-09 \\
\hline
\end{tabular}


Table A-2. (continued).

\begin{tabular}{|c|c|c|c|c|c|c|c|c|c|c|c|}
\hline $\begin{array}{c}\text { Field Sample } \\
\text { Number }\end{array}$ & Location & Depth & Compound & $\begin{array}{c}\text { Sample } \\
\text { Result }\end{array}$ & $\begin{array}{l}\text { Sample } \\
\text { Error }\end{array}$ & $\begin{array}{c}\text { Result } \\
\text { Qualifier }\end{array}$ & $\begin{array}{c}\text { Validation } \\
\text { Flag }\end{array}$ & $\begin{array}{c}\text { Sample } \\
\text { Units }\end{array}$ & $\begin{array}{c}\text { Date Sample } \\
\text { Collected }\end{array}$ & MDA & L\&V Report Number \\
\hline GWA04401VL & HIGHWAY 3 & 538 & 1,1-Dichloroethane & 1 & & $U$ & & UG/L & $06 / 09 / 2009$ & & HCJ-148-09 \\
\hline GWA04401VL & HIGHWAY 3 & 538 & 2-Butanone & 5 & & U & & UG/L & $06 / 09 / 2009$ & & HCJ-148-09 \\
\hline GWA04401VL & HIGHWAY 3 & 538 & cis-1,2-Dichloroethylene & 1 & & $\mathrm{U}$ & & UG/L & 06/09/2009 & & HCJ-148-09 \\
\hline GWA04401VL & HIGHWAY 3 & 538 & Chloroform & 1 & & $\mathrm{U}$ & & UG/L & 06/09/2009 & & HCJ-148-09 \\
\hline GWA04401VL & HIGHWAY 3 & 538 & 1,1,1-Trichloroethane & 1 & & U & & UG/L & 06/09/2009 & & HCJ-148-09 \\
\hline GWA04401VL & HIGHWAY 3 & 538 & Cyclohexane & 1 & & U & & UG/L & $06 / 09 / 2009$ & & HCJ-148-09 \\
\hline GWA04401VL & HIGHWAY 3 & 538 & Carbon tetrachloride & 1 & & $\mathrm{U}$ & & UG/L & $06 / 09 / 2009$ & & HCJ-148-09 \\
\hline GWA04401VL & HIGHWAY 3 & 538 & 1,2-Dichloroethane & 1 & & U & & UG/L & 06/09/2009 & & HCJ-148-09 \\
\hline GWA04401VL & HIGHWAY 3 & 538 & Benzene & 1 & & U & & UG/L & $06 / 09 / 2009$ & & HCJ-148-09 \\
\hline GWA04401VL & HIGHWAY 3 & 538 & Trichloroethylene & 1 & & $\mathrm{U}$ & & UG/L & 06/09/2009 & & HCJ-148-09 \\
\hline GWA04401VL & HIGHWAY 3 & 538 & 1,2-Dichloropropane & 1 & & $\mathrm{U}$ & & UG/L & $06 / 09 / 2009$ & & HCJ-148-09 \\
\hline GWA04401VL & HIGHWAY 3 & 538 & Methyl cyclohexane & 1 & & U & & UG/L & 06/09/2009 & & HCJ-148-09 \\
\hline GWA04401VL & HIGHWAY 3 & 538 & Bromodichloromethane & 1 & & U & & UG/L & 06/09/2009 & & HCJ-148-09 \\
\hline GWA04401VL & HIGHWAY 3 & 538 & 4-Methyl-2-pentanone & 5 & & $\mathrm{u}$ & & UG/L & $06 / 09 / 2009$ & & HCJ-148-09 \\
\hline GWA04401VL & HIGHWAY 3 & 538 & cis-1,3-Dichloropropylene & 1 & & U & & UG/L & 06/09/2009 & & HCJ-148-09 \\
\hline GWA04401VL & HIGHWAY 3 & 538 & Toluene & 0.56 & & $\mathrm{~J}$ & $\mathrm{~J}$ & UG/L & $06 / 09 / 2009$ & & HCJ-148-09 \\
\hline GWA04401VL & HIGHWAY 3 & 538 & trans-1,3-Dichloropropylene & 1 & & $u$ & & UG/L & $06 / 09 / 2009$ & & HCJ-148-09 \\
\hline GWA04401VL & HIGHWAY 3 & 538 & 1,1,2-Trichloroethane & 1 & & $\mathrm{U}$ & & UG/L & $06 / 09 / 2009$ & & HCJ-148-09 \\
\hline GWA04401VL & HIGHWAY 3 & 538 & 2-Hexanone & 5 & & U & & UG/L & 06/09/2009 & & HCJ-148-09 \\
\hline GWA04401VL & HIGHWAY 3 & 538 & Tetrachloroethylene & 1 & & U & & UG/L & 06/09/2009 & & HCJ-148-09 \\
\hline GWA04401VL & HIGHWAY 3 & 538 & Dibromochloromethane & 1 & & $\mathrm{U}$ & & UG/L & 06/09/2009 & & HCJ-148-09 \\
\hline GWA04401VL & HIGHWAY 3 & 538 & 1,2-Dibromoethane & 1 & & U & & UG/L & $06 / 09 / 2009$ & & HCJ-148-09 \\
\hline GWA04401VL & HIGHWAY 3 & 538 & Chlorobenzene & 1 & & U & & UG/L & $06 / 09 / 2009$ & & HCJ-148-09 \\
\hline GWA04401VL & HIGHWAY 3 & 538 & Ethylbenzene & 1 & & U & & UG/L & 06/09/2009 & & HCJ-148-09 \\
\hline GWA04401VL & HIGHWAY 3 & 538 & Styrene & 1 & & $\mathrm{u}$ & & UG/L & $06 / 09 / 2009$ & & HCJ-148-09 \\
\hline GWA04401VL & HIGHWAY 3 & 538 & Bromoform & 1 & & U & & UG/L & 06/09/2009 & & HCJ-148-09 \\
\hline GWA04401VL & HIGHWAY 3 & 538 & 1,1,2,2-Tetrachloroethane & 1 & & $u$ & & UG/L & 06/09/2009 & & HCJ-148-09 \\
\hline GWA04401VL & HIGHWAY 3 & 538 & Isopropylbenzene & 1 & & $\mathrm{u}$ & & UG/L & $06 / 09 / 2009$ & & HCJ-148-09 \\
\hline GWA04401VL & HIGHWAY 3 & 538 & 1,3-Dichlorobenzene & 1 & & $\mathrm{U}$ & & UG/L & $06 / 09 / 2009$ & & HCJ-148-09 \\
\hline GWA04401VL & HIGHWAY 3 & 538 & 1,4-Dichlorobenzene & 1 & & $\mathrm{U}$ & & UG/L & 06/09/2009 & & HCJ-148-09 \\
\hline GWA04401VL & HIGHWAY 3 & 538 & 1,2-Dibromo-3-chloropropane & 1 & & $u$ & & UG/L & $06 / 09 / 2009$ & & HCJ-148-09 \\
\hline GWA04401VL & HIGHWAY 3 & 538 & Trichlorotrifluoroethane & 5 & & $\mathrm{U}$ & & UG/L & 06/09/2009 & & HCJ-148-09 \\
\hline GWA04401VL & HIGHWAY 3 & 538 & Xylene (Total) & 1 & & $\mathrm{U}$ & & UG/L & 06/09/2009 & & HCJ-148-09 \\
\hline GWA04401VL & HIGHWAY 3 & 538 & 1,2,4-Trichlorobenzene & 1 & & $U$ & & UG/L & 06/09/2009 & & HCJ-148-09 \\
\hline GWA04401VL & HIGHWAY 3 & 538 & 1,2-Dichlorobenzene & 1 & & $\mathrm{u}$ & & UG/L & 06/09/2009 & & HCJ-148-09 \\
\hline GWA04401R8 & HIGHWAY 3 & 538 & Tritium & $-7.13 \mathrm{E}+01$ & $8.59 \mathrm{E}+01$ & & $\mathrm{U}$ & $\mathrm{PCl} / \mathrm{L}$ & $06 / 09 / 2009$ & $3.06 \mathrm{E}+02$ & BAM-119-09 \\
\hline GWA04401RH & HIGHWAY 3 & 538 & Gross Alpha & $1.92 \mathrm{E}+00$ & $6.75 \mathrm{E}-01$ & & $\mathrm{~J}$ & $\mathrm{PCI} / \mathrm{L}$ & 06/09/2009 & $1.28 \mathrm{E}+00$ & BAM-120-09 \\
\hline GWA04401RH & HIGHWAY 3 & 538 & Gross Beta & $1.62 \mathrm{E}+00$ & $5.62 \mathrm{E}-01$ & & $\mathrm{~J}$ & $\mathrm{PCl} / \mathrm{L}$ & 06/09/2009 & $1.52 \mathrm{E}+00$ & BAM-120-09 \\
\hline GWA04401RH & HIGHWAY 3 & 538 & Strontium-90 & 1.11E-01 & 1.03E-01 & & $\mathrm{U}$ & $\mathrm{PCl} / \mathrm{L}$ & 06/09/2009 & $3.51 \mathrm{E}-01$ & BAM-120-09 \\
\hline GWA04401RH & HIGHWAY 3 & 538 & Technetium-99 & $-2.98 \mathrm{E}+00$ & $1.60 \mathrm{E}+00$ & & $\mathrm{U}$ & $\mathrm{PCl} / \mathrm{L}$ & 06/09/2009 & $5.88 \mathrm{E}+00$ & BAM-120-09 \\
\hline GWA04401UX & HIGHWAY 3 & 538 & lodine-129 & 2.21E-02 & $2.22 \mathrm{E}-02$ & & $U$ & $\mathrm{PCl} / \mathrm{L}$ & 06/09/2009 & 8.12E-02 & BAM-118-09 \\
\hline GWA05301A1 & FIELD BLANK & NA & Alkalinity, Total as $\mathrm{CaCO} 3$ & 1.04 & & $\mathrm{~J}$ & $\mathrm{U}$ & $M G / L$ & $06 / 17 / 2009$ & & HCJ-156-09 \\
\hline GWA05301AN & FIELD BLANK & NA & Bromide & 0 & & U & & $M G / L$ & $06 / 17 / 2009$ & & HCJ-158-09 \\
\hline GWA05301AN & FIELD BLANK & NA & Chloride & 0 & & $\mathrm{U}$ & & $M G / L$ & $06 / 17 / 2009$ & & HCJ-158-09 \\
\hline
\end{tabular}


Table A-2. (continued).

\begin{tabular}{|c|c|c|c|c|c|c|c|c|c|c|c|}
\hline $\begin{array}{c}\text { Field Sample } \\
\text { Number }\end{array}$ & Location & Depth & Compound & $\begin{array}{c}\text { Sample } \\
\text { Result }\end{array}$ & $\begin{array}{c}\text { Sample } \\
\text { Error }\end{array}$ & $\begin{array}{c}\text { Result } \\
\text { Qualifier }\end{array}$ & $\begin{array}{c}\text { Validation } \\
\text { Flag }\end{array}$ & $\begin{array}{c}\text { Sample } \\
\text { Units }\end{array}$ & $\begin{array}{c}\text { Date Sample } \\
\text { Collected }\end{array}$ & MDA & L\&V Report Number \\
\hline GWA05301AN & FIELD BLANK & NA & Fluoride & 0 & & $U$ & & $M G / L$ & $06 / 17 / 2009$ & & HCJ-158-09 \\
\hline GWA05301AN & FIELD BLANK & NA & Sulfate & 0 & & U & & $M G / L$ & 06/17/2009 & & HCJ-158-09 \\
\hline GWA05301N2 & FIELD BLANK & NA & Nitrogen, Nitrate/Nitrite & 0.06 & & $\mathrm{~J}$ & $\mathrm{U}$ & $M G / L$ & $06 / 17 / 2009$ & & HCJ-154-09 \\
\hline GWA05302A1 & FIELD BLANK & NA & Alkalinity, Total as $\mathrm{CaCO} 3$ & 1.04 & & $\mathrm{~J}$ & $\mathrm{U}$ & $M G / L$ & 07/02/2009 & & HCJ-168-09 \\
\hline GWA05302AN & FIELD BLANK & NA & Bromide & 0 & & U & & $M G / L$ & 07/02/2009 & & HCJ-170-09 \\
\hline GWA05302AN & FIELD BLANK & NA & Chloride & 0 & & $u$ & & $M G / L$ & 07/02/2009 & & HCJ-170-09 \\
\hline GWA05302AN & FIELD BLANK & NA & Fluoride & 0 & & $\mathrm{U}$ & & $M G / L$ & 07/02/2009 & & HCJ-170-09 \\
\hline GWA05302AN & FIELD BLANK & NA & Sulfate & 0 & & U & & $M G / L$ & 07/02/2009 & & HCJ-170-09 \\
\hline GWA05303AN & FIELD BLANK & NA & Bromide & 0 & & U & & $M G / L$ & 07/13/2009 & & HCJ-170-09 \\
\hline GWA05303AN & FIELD BLANK & NA & Chloride & 0 & & $\mathrm{u}$ & & $M G / L$ & 07/13/2009 & & HCJ-170-09 \\
\hline GWA05303AN & FIELD BLANK & NA & Fluoride & 0 & & $\mathrm{U}$ & & $M G / L$ & 07/13/2009 & & HCJ-170-09 \\
\hline GWA05303AN & FIELD BLANK & NA & Sulfate & 0 & & U & & $M G / L$ & 07/13/2009 & & HCJ-170-09 \\
\hline GWA05302N2 & FIELD BLANK & NA & Nitrogen, Nitrate/Nitrite & 0.00331 & & U & & MG/L & 07/02/2009 & & HCJ-171-09 \\
\hline GWA05303N2 & FIELD BLANK & NA & Nitrogen, Nitrate/Nitrite & 0.00992 & & $\mathrm{u}$ & & $M G / L$ & 07/13/2009 & & HCJ-171-09 \\
\hline GWA05303A1 & FIELD BLANK & NA & Alkalinity, Total as $\mathrm{CaCO} 3$ & 0.521 & & U & & $M G / L$ & 07/13/2009 & & HCJ-169-09 \\
\hline GWA053012X & FIELD BLANK & NA & Aluminum & 15 & & U & & UG/L & 06/17/2009 & & HCJ-160-09 \\
\hline GWA053012X & FIELD BLANK & NA & Antimony & 1 & & $\mathrm{u}$ & & UG/L & $06 / 17 / 2009$ & & HCJ-160-09 \\
\hline GWA053012X & FIELD BLANK & NA & Arsenic & 1.6 & & $\mathrm{U}$ & & UG/L & 06/17/2009 & & HCJ-160-09 \\
\hline GWA053012X & FIELD BLANK & NA & Barium & 0.6 & & U & & UG/L & 06/17/2009 & & HCJ-160-09 \\
\hline GWA053012X & FIELD BLANK & NA & Beryllium & 0.1 & & U & & UG/L & 06/17/2009 & & HCJ-160-09 \\
\hline GWA053012X & FIELD BLANK & NA & Cadmium & 0.11 & & $\mathrm{u}$ & & UG/L & 06/17/2009 & & HCJ-160-09 \\
\hline GWA053012X & FIELD BLANK & NA & Calcium & 81 & & B & U & UG/L & 06/17/2009 & & HCJ-160-09 \\
\hline GWA053012X & FIELD BLANK & NA & Chromium & 2 & & U & & UG/L & 06/17/2009 & & HCJ-160-09 \\
\hline GWA053012X & FIELD BLANK & NA & Cobalt & 0.1 & & $U$ & & UG/L & 06/17/2009 & & HCJ-160-09 \\
\hline GWA053012X & FIELD BLANK & NA & Copper & 13.2 & & B & & UG/L & 06/17/2009 & & HCJ-160-09 \\
\hline GWA053012X & FIELD BLANK & NA & Iron & 33 & & U & & UG/L & 06/17/2009 & & HCJ-160-09 \\
\hline GWA053012X & FIELD BLANK & NA & Lead & 0.711 & & B & & UG/L & 06/17/2009 & & HCJ-160-09 \\
\hline GWA053012X & FIELD BLANK & NA & Magnesium & 5.2 & & U & & UG/L & $06 / 17 / 2009$ & & HCJ-160-09 \\
\hline GWA053012X & FIELD BLANK & NA & Manganese & 1 & & U & & UG/L & 06/17/2009 & & HCJ-160-09 \\
\hline GWA053012X & FIELD BLANK & NA & Mercury & 0.066 & & $U$ & & UG/L & 06/17/2009 & & HCJ-160-09 \\
\hline GWA053012X & FIELD BLANK & NA & Nickel & 0.5 & & $U$ & & UG/L & 06/17/2009 & & HCJ-160-09 \\
\hline GWA053012X & FIELD BLANK & NA & Potassium & 80 & & U & & UG/L & 06/17/2009 & & HCJ-160-09 \\
\hline GWA053012X & FIELD BLANK & NA & Selenium & 1 & & $U$ & & UG/L & 06/17/2009 & & HCJ-160-09 \\
\hline GWA053012X & FIELD BLANK & NA & Silver & 0.2 & & U & & UG/L & 06/17/2009 & & HCJ-160-09 \\
\hline GWA053012X & FIELD BLANK & NA & Sodium & 80 & & U & & UG/L & 06/17/2009 & & HCJ-160-09 \\
\hline GWA053012X & FIELD BLANK & NA & Strontium & 2 & & U & & UG/L & 06/17/2009 & & HCJ-160-09 \\
\hline GWA053012X & FIELD BLANK & NA & Thallium & 0.3 & & U & & UG/L & 06/17/2009 & & HCJ-160-09 \\
\hline GWA053012X & FIELD BLANK & NA & Uranium & 0.05 & & $u$ & & UG/L & 06/17/2009 & & HCJ-160-09 \\
\hline GWA053012X & FIELD BLANK & NA & Vanadium & 3 & & U & & UG/L & 06/17/2009 & & HCJ-160-09 \\
\hline GWA053012X & FIELD BLANK & NA & Zinc & 42.9 & & & & UG/L & 06/17/2009 & & HCJ-160-09 \\
\hline GWA053022X & FIELD BLANK & NA & Aluminum & 15 & & $U$ & & UG/L & 07/02/2009 & & HCJ-172-09 \\
\hline GWA053022X & FIELD BLANK & NA & Antimony & 1 & & U & & UG/L & 07/02/2009 & & HCJ-172-09 \\
\hline GWA053022X & FIELD BLANK & NA & Arsenic & 1.6 & & U & & UG/L & 07/02/2009 & & HCJ-172-09 \\
\hline GWA053022X & FIELD BLANK & NA & Barium & 0.6 & & $U$ & & UG/L & 07/02/2009 & & HCJ-172-09 \\
\hline
\end{tabular}


Table A-2. (continued).

\begin{tabular}{|c|c|c|c|c|c|c|c|c|c|c|c|}
\hline $\begin{array}{c}\text { Field Sample } \\
\text { Number }\end{array}$ & Location & Depth & Compound & $\begin{array}{c}\text { Sample } \\
\text { Result }\end{array}$ & $\begin{array}{l}\text { Sample } \\
\text { Error }\end{array}$ & $\begin{array}{c}\text { Result } \\
\text { Qualifier }\end{array}$ & $\begin{array}{l}\text { Validation } \\
\text { Flag }\end{array}$ & $\begin{array}{c}\text { Sample } \\
\text { Units }\end{array}$ & $\begin{array}{c}\text { Date Sample } \\
\text { Collected }\end{array}$ & MDA & L\&V Report Number \\
\hline GWA053022X & FIELD BLANK & NA & Beryllium & 0.1 & & $\mathrm{U}$ & & UG/L & $07 / 02 / 2009$ & & HCJ-172-09 \\
\hline GWA053022X & FIELD BLANK & NA & Cadmium & 0.11 & & U & & UG/L & $07 / 02 / 2009$ & & HCJ-172-09 \\
\hline GWA053022X & FIELD BLANK & NA & Calcium & 65 & & $\mathrm{U}$ & & UG/L & 07/02/2009 & & HCJ-172-09 \\
\hline GWA053022X & FIELD BLANK & NA & Chromium & 2 & & $\mathrm{U}$ & & UG/L & 07/02/2009 & & HCJ-172-09 \\
\hline GWA053022X & FIELD BLANK & NA & Cobalt & 0.1 & & $\mathrm{U}$ & & UG/L & 07/02/2009 & & HCJ-172-09 \\
\hline GWA053022X & FIELD BLANK & NA & Copper & 0.734 & & B & & UG/L & 07/02/2009 & & HCJ-172-09 \\
\hline GWA053022X & FIELD BLANK & NA & Iron & 33 & & U & & UG/L & 07/02/2009 & & HCJ-172-09 \\
\hline GWA053022X & FIELD BLANK & NA & Lead & 0.5 & & U & & UG/L & 07/02/2009 & & HCJ-172-09 \\
\hline GWA053022X & FIELD BLANK & NA & Magnesium & 5.2 & & $\mathrm{U}$ & & UG/L & $07 / 02 / 2009$ & & HCJ-172-09 \\
\hline GWA053022X & FIELD BLANK & NA & Manganese & 1 & & $\mathrm{U}$ & & UG/L & 07/02/2009 & & HCJ-172-09 \\
\hline GWA053022X & FIELD BLANK & NA & Mercury & 0.066 & & $\mathrm{U}$ & & UG/L & 07/02/2009 & & HCJ-172-09 \\
\hline GWA053022X & FIELD BLANK & NA & Nickel & 0.5 & & $\mathrm{U}$ & & UG/L & 07/02/2009 & & HCJ-172-09 \\
\hline GWA053022X & FIELD BLANK & NA & Potassium & 80 & & $U$ & & UG/L & $07 / 02 / 2009$ & & HCJ-172-09 \\
\hline GWA053022X & FIELD BLANK & NA & Selenium & 1 & & $\mathrm{U}$ & & UG/L & 07/02/2009 & & HCJ-172-09 \\
\hline GWA053022X & FIELD BLANK & NA & Silver & 0.2 & & U & & UG/L & 07/02/2009 & & HCJ-172-09 \\
\hline GWA053022X & FIELD BLANK & NA & Sodium & 80 & & UE & UJ & UG/L & $07 / 02 / 2009$ & & HCJ-172-09 \\
\hline GWA053022X & FIELD BLANK & NA & Strontium & 2 & & $U$ & & UG/L & 07/02/2009 & & HCJ-172-09 \\
\hline GWA053022X & FIELD BLANK & NA & Thallium & 0.3 & & $U$ & & UG/L & 07/02/2009 & & HCJ-172-09 \\
\hline GWA053022X & FIELD BLANK & NA & Uranium & 0.05 & & $\mathrm{U}$ & & UG/L & 07/02/2009 & & HCJ-172-09 \\
\hline GWA053022X & FIELD BLANK & NA & Vanadium & 3 & & $U$ & & UG/L & $07 / 02 / 2009$ & & HCJ-172-09 \\
\hline GWA053022X & FIELD BLANK & NA & Zinc & 3 & & $U$ & & UG/L & 07/02/2009 & & HCJ-172-09 \\
\hline GWA053032X & FIELD BLANK & NA & Aluminum & 15 & & $\mathrm{U}$ & & UG/L & 07/13/2009 & & HCJ-172-09 \\
\hline GWA053032X & FIELD BLANK & NA & Antimony & 1 & & $\mathrm{U}$ & & UG/L & $07 / 13 / 2009$ & & HCJ-172-09 \\
\hline GWA053032X & FIELD BLANK & NA & Arsenic & 2.48 & & $B$ & $U$ & UG/L & 07/13/2009 & & HCJ-172-09 \\
\hline GWA053032X & FIELD BLANK & NA & Barium & 0.6 & & $U$ & & UG/L & $07 / 13 / 2009$ & & HCJ-172-09 \\
\hline GWA053032X & FIELD BLANK & NA & Beryllium & 0.1 & & $\mathrm{U}$ & & UG/L & 07/13/2009 & & HCJ-172-09 \\
\hline GWA053032X & FIELD BLANK & NA & Cadmium & 0.11 & & $U$ & & UG/L & 07/13/2009 & & HCJ-172-09 \\
\hline GWA053032X & FIELD BLANK & NA & Calcium & 66.5 & & B & & UG/L & 07/13/2009 & & HCJ-172-09 \\
\hline GWA053032X & FIELD BLANK & NA & Chromium & 2 & & $U$ & & UG/L & $07 / 13 / 2009$ & & HCJ-172-09 \\
\hline GWA053032X & FIELD BLANK & NA & Cobalt & 0.1 & & $\mathrm{U}$ & & UG/L & 07/13/2009 & & HCJ-172-09 \\
\hline GWA053032X & FIELD BLANK & NA & Copper & 2.28 & & $B$ & & UG/L & 07/13/2009 & & HCJ-172-09 \\
\hline GWA053032X & FIELD BLANK & NA & Iron & 33 & & $U$ & & UG/L & $07 / 13 / 2009$ & & HCJ-172-09 \\
\hline GWA053032X & FIELD BLANK & NA & Lead & 0.5 & & $\mathrm{U}$ & & UG/L & $07 / 13 / 2009$ & & HCJ-172-09 \\
\hline GWA053032X & FIELD BLANK & NA & Magnesium & 5.2 & & $U$ & & UG/L & 07/13/2009 & & HCJ-172-09 \\
\hline GWA053032X & FIELD BLANK & NA & Manganese & 1 & & $U$ & & UG/L & 07/13/2009 & & HCJ-172-09 \\
\hline GWA053032X & FIELD BLANK & NA & Mercury & 0.066 & & U & & UG/L & 07/13/2009 & & HCJ-172-09 \\
\hline GWA053032X & FIELD BLANK & NA & Nickel & 0.5 & & $\mathrm{U}$ & & UG/L & 07/13/2009 & & HCJ-172-09 \\
\hline GWA053032X & FIELD BLANK & NA & Potassium & 80 & & $U$ & & UG/L & 07/13/2009 & & HCJ-172-09 \\
\hline GWA053032X & FIELD BLANK & NA & Selenium & 1 & & $\mathrm{U}$ & & UG/L & 07/13/2009 & & HCJ-172-09 \\
\hline GWA053032X & FIELD BLANK & NA & Silver & 0.2 & & $\mathrm{U}$ & & UG/L & 07/13/2009 & & HCJ-172-09 \\
\hline GWA053032X & FIELD BLANK & NA & Sodium & 80 & & UE & & UG/L & 07/13/2009 & & HCJ-172-09 \\
\hline GWA053032X & FIELD BLANK & NA & Strontium & 2 & & $U$ & & UG/L & 07/13/2009 & & HCJ-172-09 \\
\hline GWA053032X & FIELD BLANK & NA & Thallium & 0.3 & & $U$ & & UG/L & 07/13/2009 & & HCJ-172-09 \\
\hline GWA053032X & FIELD BLANK & NA & Uranium & 0.05 & & $U$ & & UG/L & 07/13/2009 & & HCJ-172-09 \\
\hline
\end{tabular}


Table A-2. (continued).

\begin{tabular}{|c|c|c|c|c|c|c|c|c|c|c|c|}
\hline $\begin{array}{c}\text { Field Sample } \\
\text { Number }\end{array}$ & Location & Depth & Compound & $\begin{array}{c}\text { Sample } \\
\text { Result }\end{array}$ & $\begin{array}{l}\text { Sample } \\
\text { Error }\end{array}$ & $\begin{array}{c}\text { Result } \\
\text { Qualifier }\end{array}$ & $\begin{array}{l}\text { Validation } \\
\text { Flag }\end{array}$ & $\begin{array}{c}\text { Sample } \\
\text { Units }\end{array}$ & $\begin{array}{c}\text { Date Sample } \\
\text { Collected }\end{array}$ & MDA & L\&V Report Number \\
\hline GWA053032X & FIELD BLANK & NA & Vanadium & 3 & & $\mathrm{U}$ & & UG/L & $07 / 13 / 2009$ & & HCJ-172-09 \\
\hline GWA053032X & FIELD BLANK & NA & Zinc & 3 & & $\mathrm{U}$ & & UG/L & 07/13/2009 & & HCJ-172-09 \\
\hline GWA05401VL & TRIP BLANK & NA & Dichlorodifluoromethane & 1 & & $\mathrm{U}$ & & UG/L & 06/02/2009 & & HCJ-148-09 \\
\hline GWA05401VL & TRIP BLANK & NA & Chloromethane & 1 & & $\mathrm{U}$ & & UG/L & 06/02/2009 & & HCJ-148-09 \\
\hline GWA05401VL & TRIP BLANK & NA & Vinyl Chloride & 1 & & $\mathrm{U}$ & & UG/L & 06/02/2009 & & HCJ-148-09 \\
\hline GWA05401VL & TRIP BLANK & NA & Bromomethane & 1 & & $\mathrm{U}$ & & UG/L & 06/02/2009 & & HCJ-148-09 \\
\hline GWA05401VL & TRIP BLANK & NA & Chloroethane & 1 & & $\mathrm{U}$ & & UG/L & 06/02/2009 & & HCJ-148-09 \\
\hline GWA05401VL & TRIP BLANK & NA & Trichlorofluoromethane & 1 & & $\mathrm{U}$ & & UG/L & 06/02/2009 & & HCJ-148-09 \\
\hline GWA05401VL & TRIP BLANK & NA & Acetone & 5 & & $\mathrm{U}$ & & UG/L & 06/02/2009 & & HCJ-148-09 \\
\hline GWA05401VL & TRIP BLANK & NA & 1,1-Dichloroethene & 1 & & $\mathrm{U}$ & & UG/L & 06/02/2009 & & HCJ-148-09 \\
\hline GWA05401VL & TRIP BLANK & NA & Methyl acetate & 5 & & $\mathrm{U}$ & & UG/L & 06/02/2009 & & HCJ-148-09 \\
\hline GWA05401VL & TRIP BLANK & NA & Methylene Chloride & 5 & & $\mathrm{U}$ & & UG/L & 06/02/2009 & & HCJ-148-09 \\
\hline GWA05401VL & TRIP BLANK & NA & Carbon disulfide & 5 & & $\mathrm{U}$ & & UG/L & 06/02/2009 & & HCJ-148-09 \\
\hline GWA05401VL & TRIP BLANK & NA & Tert-butyl methyl ether & 1 & & $\mathrm{U}$ & & UG/L & 06/02/2009 & & HCJ-148-09 \\
\hline GWA05401VL & TRIP BLANK & NA & trans-1,2-Dichloroethylene & 1 & & $\mathrm{U}$ & & UG/L & 06/02/2009 & & HCJ-148-09 \\
\hline GWA05401VL & TRIP BLANK & NA & 1,1-Dichloroethane & 1 & & $\mathrm{U}$ & & UG/L & 06/02/2009 & & HCJ-148-09 \\
\hline GWA05401VL & TRIP BLANK & NA & 2-Butanone & 5 & & $\mathrm{U}$ & & UG/L & 06/02/2009 & & HCJ-148-09 \\
\hline GWA05401VL & TRIP BLANK & NA & cis-1,2-Dichloroethylene & 1 & & $\mathrm{U}$ & & UG/L & 06/02/2009 & & HCJ-148-09 \\
\hline GWA05401VL & TRIP BLANK & NA & Chloroform & 2.96 & & & & UG/L & 06/02/2009 & & HCJ-148-09 \\
\hline GWA05401VL & TRIP BLANK & NA & 1,1,1-Trichloroethane & 1 & & $\mathrm{U}$ & & UG/L & 06/02/2009 & & HCJ-148-09 \\
\hline GWA05401VL & TRIP BLANK & NA & Cyclohexane & 1 & & $\mathrm{U}$ & & UG/L & 06/02/2009 & & HCJ-148-09 \\
\hline GWA05401VL & TRIP BLANK & NA & Carbon tetrachloride & 1 & & $\mathrm{U}$ & & UG/L & 06/02/2009 & & HCJ-148-09 \\
\hline GWA05401VL & TRIP BLANK & NA & 1,2-Dichloroethane & 1 & & $\mathrm{U}$ & & UG/L & 06/02/2009 & & HCJ-148-09 \\
\hline GWA05401VL & TRIP BLANK & NA & Benzene & 1 & & $\mathrm{U}$ & & UG/L & 06/02/2009 & & HCJ-148-09 \\
\hline GWA05401VL & TRIP BLANK & NA & Trichloroethylene & 1 & & $\mathrm{U}$ & & UG/L & 06/02/2009 & & HCJ-148-09 \\
\hline GWA05401VL & TRIP BLANK & NA & 1,2-Dichloropropane & 1 & & $\mathrm{U}$ & & UG/L & 06/02/2009 & & HCJ-148-09 \\
\hline GWA05401VL & TRIP BLANK & NA & Methyl cyclohexane & 1 & & $\mathrm{U}$ & & UG/L & 06/02/2009 & & HCJ-148-09 \\
\hline GWA05401VL & TRIP BLANK & NA & Bromodichloromethane & 1 & & $\mathrm{U}$ & & UG/L & 06/02/2009 & & HCJ-148-09 \\
\hline GWA05401VL & TRIP BLANK & NA & 4-Methyl-2-pentanone & 5 & & $\mathrm{U}$ & & UG/L & 06/02/2009 & & HCJ-148-09 \\
\hline GWA05401VL & TRIP BLANK & NA & cis-1,3-Dichloropropylene & 1 & & $\mathrm{U}$ & & UG/L & 06/02/2009 & & HCJ-148-09 \\
\hline GWA05401VL & TRIP BLANK & NA & Toluene & 1 & & $\mathrm{U}$ & & UG/L & 06/02/2009 & & HCJ-148-09 \\
\hline GWA05401VL & TRIP BLANK & NA & trans-1,3-Dichloropropylene & 1 & & $\mathrm{U}$ & & UG/L & 06/02/2009 & & HCJ-148-09 \\
\hline GWA05401VL & TRIP BLANK & NA & 1,1,2-Trichloroethane & 1 & & $\mathrm{U}$ & & UG/L & 06/02/2009 & & HCJ-148-09 \\
\hline GWA05401VL & TRIP BLANK & NA & 2-Hexanone & 5 & & $\mathrm{U}$ & & UG/L & 06/02/2009 & & HCJ-148-09 \\
\hline GWA05401VL & TRIP BLANK & NA & Tetrachloroethylene & 1 & & $\mathrm{U}$ & & UG/L & 06/02/2009 & & HCJ-148-09 \\
\hline GWA05401VL & TRIP BLANK & NA & Dibromochloromethane & 1 & & $\mathrm{U}$ & & UG/L & 06/02/2009 & & HCJ-148-09 \\
\hline GWA05401VL & TRIP BLANK & NA & 1,2-Dibromoethane & 1 & & $\mathrm{U}$ & & UG/L & 06/02/2009 & & HCJ-148-09 \\
\hline GWA05401VL & TRIP BLANK & NA & Chlorobenzene & 1 & & $\mathrm{U}$ & & UG/L & 06/02/2009 & & HCJ-148-09 \\
\hline GWA05401VL & TRIP BLANK & NA & Ethylbenzene & 1 & & $\mathrm{U}$ & & UG/L & 06/02/2009 & & HCJ-148-09 \\
\hline GWA05401VL & TRIP BLANK & NA & Styrene & 1 & & $\mathrm{U}$ & & UG/L & 06/02/2009 & & HCJ-148-09 \\
\hline GWA05401VL & TRIP BLANK & NA & Bromoform & 1 & & $\mathrm{U}$ & & UG/L & 06/02/2009 & & HCJ-148-09 \\
\hline GWA05401VL & TRIP BLANK & NA & 1,1,2,2-Tetrachloroethane & 1 & & $\mathrm{U}$ & & UG/L & 06/02/2009 & & HCJ-148-09 \\
\hline GWA05401VL & TRIP BLANK & NA & Isopropylbenzene & 1 & & U & & UG/L & 06/02/2009 & & HCJ-148-09 \\
\hline GWA05401VL & TRIP BLANK & NA & 1,3-Dichlorobenzene & 1 & & $\mathrm{U}$ & & UG/L & 06/02/2009 & & HCJ-148-09 \\
\hline
\end{tabular}


Table A-2. (continued).

\begin{tabular}{|c|c|c|c|c|c|c|c|c|c|c|c|}
\hline $\begin{array}{c}\text { Field Sample } \\
\text { Number }\end{array}$ & Location & Depth & Compound & $\begin{array}{c}\text { Sample } \\
\text { Result }\end{array}$ & $\begin{array}{c}\text { Sample } \\
\text { Error }\end{array}$ & $\begin{array}{c}\text { Result } \\
\text { Qualifier }\end{array}$ & $\begin{array}{c}\text { Validation } \\
\text { Flag }\end{array}$ & $\begin{array}{c}\text { Sample } \\
\text { Units }\end{array}$ & $\begin{array}{c}\text { Date Sample } \\
\text { Collected }\end{array}$ & MDA & L\&V Report Number \\
\hline GWA05401VL & TRIP BLANK & NA & 1,4-Dichlorobenzene & 1 & & $\mathrm{U}$ & & UG/L & $06 / 02 / 2009$ & & HCJ-148-09 \\
\hline GWA05401VL & TRIP BLANK & NA & 1,2-Dibromo-3-chloropropane & 1 & & $\mathrm{U}$ & & UG/L & 06/02/2009 & & HCJ-148-09 \\
\hline GWA05401VL & TRIP BLANK & NA & Trichlorotrifluoroethane & 5 & & $\mathrm{U}$ & & UG/L & 06/02/2009 & & HCJ-148-09 \\
\hline GWA05401VL & TRIP BLANK & NA & Xylene (Total) & 1 & & $\mathrm{U}$ & & UG/L & 06/02/2009 & & HCJ-148-09 \\
\hline GWA05401VL & TRIP BLANK & NA & 1,2,4-Trichlorobenzene & 1 & & $\mathrm{U}$ & & UG/L & 06/02/2009 & & HCJ-148-09 \\
\hline GWA05401VL & TRIP BLANK & NA & 1,2-Dichlorobenzene & 1 & & $\mathrm{U}$ & & UG/L & 06/02/2009 & & HCJ-148-09 \\
\hline GWA05402VL & TRIP BLANK & NA & Dichlorodifluoromethane & 1 & & $\mathrm{U}$ & & UG/L & 06/09/2009 & & HCJ-148-09 \\
\hline GWA05402VL & TRIP BLANK & NA & Chloromethane & 1 & & $\mathrm{U}$ & & UG/L & 06/09/2009 & & HCJ-148-09 \\
\hline GWA05402VL & TRIP BLANK & NA & Vinyl Chloride & 1 & & $\mathrm{U}$ & & UG/L & 06/09/2009 & & HCJ-148-09 \\
\hline GWA05402VL & TRIP BLANK & NA & Bromomethane & 1 & & $\mathrm{U}$ & & UG/L & 06/09/2009 & & HCJ-148-09 \\
\hline GWA05402VL & TRIP BLANK & NA & Chloroethane & 1 & & $\mathrm{U}$ & & UG/L & 06/09/2009 & & HCJ-148-09 \\
\hline GWA05402VL & TRIP BLANK & NA & Trichlorofluoromethane & 1 & & $\mathrm{U}$ & & UG/L & 06/09/2009 & & HCJ-148-09 \\
\hline GWA05402VL & TRIP BLANK & NA & Acetone & 5 & & $\mathrm{U}$ & & UG/L & 06/09/2009 & & HCJ-148-09 \\
\hline GWA05402VL & TRIP BLANK & NA & 1,1-Dichloroethene & 1 & & $\mathrm{U}$ & & UG/L & 06/09/2009 & & HCJ-148-09 \\
\hline GWA05402VL & TRIP BLANK & NA & Methyl acetate & 5 & & $\mathrm{U}$ & & UG/L & 06/09/2009 & & HCJ-148-09 \\
\hline GWA05402VL & TRIP BLANK & NA & Methylene Chloride & 5 & & $\mathrm{U}$ & & UG/L & 06/09/2009 & & HCJ-148-09 \\
\hline GWA05402VL & TRIP BLANK & NA & Carbon disulfide & 5 & & $\mathrm{U}$ & & UG/L & 06/09/2009 & & HCJ-148-09 \\
\hline GWA05402VL & TRIP BLANK & NA & Tert-butyl methyl ether & 1 & & $\mathrm{U}$ & & UG/L & 06/09/2009 & & HCJ-148-09 \\
\hline GWA05402VL & TRIP BLANK & NA & trans-1,2-Dichloroethylene & 1 & & $\mathrm{U}$ & & UG/L & 06/09/2009 & & HCJ-148-09 \\
\hline GWA05402VL & TRIP BLANK & NA & 1,1-Dichloroethane & 1 & & $\mathrm{U}$ & & UG/L & 06/09/2009 & & HCJ-148-09 \\
\hline GWA05402VL & TRIP BLANK & NA & 2-Butanone & 5 & & $\mathrm{U}$ & & UG/L & 06/09/2009 & & HCJ-148-09 \\
\hline GWA05402VL & TRIP BLANK & NA & cis-1,2-Dichloroethylene & 1 & & $\mathrm{U}$ & & UG/L & 06/09/2009 & & HCJ-148-09 \\
\hline GWA05402VL & TRIP BLANK & NA & Chloroform & 2.9 & & & $\mathrm{~J}$ & UG/L & 06/09/2009 & & HCJ-148-09 \\
\hline GWA05402VL & TRIP BLANK & NA & 1,1,1-Trichloroethane & 1 & & $U$ & & UG/L & 06/09/2009 & & HCJ-148-09 \\
\hline GWA05402VL & TRIP BLANK & NA & Cyclohexane & 1 & & $\mathrm{U}$ & & UG/L & 06/09/2009 & & HCJ-148-09 \\
\hline GWA05402VL & TRIP BLANK & NA & Carbon tetrachloride & 1 & & $\mathrm{U}$ & & UG/L & 06/09/2009 & & HCJ-148-09 \\
\hline GWA05402VL & TRIP BLANK & NA & 1,2-Dichloroethane & 1 & & $\mathrm{U}$ & & UG/L & 06/09/2009 & & HCJ-148-09 \\
\hline GWA05402VL & TRIP BLANK & NA & Benzene & 1 & & $\mathrm{U}$ & & UG/L & 06/09/2009 & & HCJ-148-09 \\
\hline GWA05402VL & TRIP BLANK & NA & Trichloroethylene & 1 & & $\mathrm{U}$ & & UG/L & 06/09/2009 & & HCJ-148-09 \\
\hline GWA05402VL & TRIP BLANK & NA & 1,2-Dichloropropane & 1 & & $\mathrm{U}$ & & UG/L & 06/09/2009 & & HCJ-148-09 \\
\hline GWA05402VL & TRIP BLANK & NA & Methyl cyclohexane & 1 & & U & & UG/L & 06/09/2009 & & HCJ-148-09 \\
\hline GWA05402VL & TRIP BLANK & NA & Bromodichloromethane & 1 & & $\mathrm{U}$ & & UG/L & 06/09/2009 & & HCJ-148-09 \\
\hline GWA05402VL & TRIP BLANK & NA & 4-Methyl-2-pentanone & 5 & & $\mathrm{U}$ & & UG/L & 06/09/2009 & & HCJ-148-09 \\
\hline GWA05402VL & TRIP BLANK & NA & cis-1,3-Dichloropropylene & 1 & & $\mathrm{U}$ & & UG/L & 06/09/2009 & & HCJ-148-09 \\
\hline GWA05402VL & TRIP BLANK & NA & Toluene & 1 & & $\mathrm{U}$ & & UG/L & 06/09/2009 & & HCJ-148-09 \\
\hline GWA05402VL & TRIP BLANK & NA & trans-1,3-Dichloropropylene & 1 & & $\mathrm{U}$ & & UG/L & 06/09/2009 & & HCJ-148-09 \\
\hline GWA05402VL & TRIP BLANK & NA & 1,1,2-Trichloroethane & 1 & & $\mathrm{U}$ & & UG/L & 06/09/2009 & & HCJ-148-09 \\
\hline GWA05402VL & TRIP BLANK & NA & 2-Hexanone & 5 & & $\mathrm{U}$ & & UG/L & 06/09/2009 & & HCJ-148-09 \\
\hline GWA05402VL & TRIP BLANK & NA & Tetrachloroethylene & 1 & & $\mathrm{U}$ & & UG/L & 06/09/2009 & & HCJ-148-09 \\
\hline GWA05402VL & TRIP BLANK & NA & Dibromochloromethane & 1 & & $\mathrm{U}$ & & UG/L & 06/09/2009 & & HCJ-148-09 \\
\hline GWA05402VL & TRIP BLANK & NA & 1,2-Dibromoethane & 1 & & $\mathrm{U}$ & & UG/L & 06/09/2009 & & HCJ-148-09 \\
\hline GWA05402VL & TRIP BLANK & NA & Chlorobenzene & 1 & & $\mathrm{U}$ & & UG/L & 06/09/2009 & & HCJ-148-09 \\
\hline GWA05402VL & TRIP BLANK & NA & Ethylbenzene & 1 & & U & & UG/L & 06/09/2009 & & HCJ-148-09 \\
\hline GWA05402VL & TRIP BLANK & NA & Styrene & 1 & & $\mathrm{U}$ & & UG/L & 06/09/2009 & & HCJ-148-09 \\
\hline
\end{tabular}


Table A-2. (continued).

\begin{tabular}{|c|c|c|c|c|c|c|c|c|c|c|c|}
\hline $\begin{array}{c}\text { Field Sample } \\
\text { Number }\end{array}$ & Location & Depth & Compound & $\begin{array}{l}\text { Sample } \\
\text { Result }\end{array}$ & $\begin{array}{c}\text { Sample } \\
\text { Error }\end{array}$ & $\begin{array}{c}\text { Result } \\
\text { Qualifier }\end{array}$ & $\begin{array}{c}\text { Validation } \\
\text { Flag }\end{array}$ & $\begin{array}{c}\text { Sample } \\
\text { Units }\end{array}$ & $\begin{array}{c}\text { Date Sample } \\
\text { Collected }\end{array}$ & MDA & L\&V Report Number \\
\hline GWA05402VL & TRIP BLANK & NA & Bromoform & 1 & & $U$ & & UG/L & $06 / 09 / 2009$ & & HCJ-148-09 \\
\hline GWA05402VL & TRIP BLANK & NA & 1,1,2,2-Tetrachloroethane & 1 & & U & & UG/L & 06/09/2009 & & HCJ-148-09 \\
\hline GWA05402VL & TRIP BLANK & NA & Isopropylbenzene & 1 & & $\mathrm{u}$ & & UG/L & 06/09/2009 & & HCJ-148-09 \\
\hline GWA05402VL & TRIP BLANK & NA & 1,3-Dichlorobenzene & 1 & & $\mathrm{U}$ & & UG/L & 06/09/2009 & & HCJ-148-09 \\
\hline GWA05402VL & TRIP BLANK & NA & 1,4-Dichlorobenzene & 1 & & U & & UG/L & $06 / 09 / 2009$ & & HCJ-148-09 \\
\hline GWA05402VL & TRIP BLANK & NA & 1,2-Dibromo-3-chloropropane & 1 & & U & & UG/L & $06 / 09 / 2009$ & & HCJ-148-09 \\
\hline GWA05402VL & TRIP BLANK & NA & Trichlorotrifluoroethane & 5 & & $\mathrm{U}$ & & UG/L & 06/09/2009 & & HCJ-148-09 \\
\hline GWA05402VL & TRIP BLANK & NA & Xylene (Total) & 1 & & U & & UG/L & 06/09/2009 & & HCJ-148-09 \\
\hline GWA05402VL & TRIP BLANK & NA & 1,2,4-Trichlorobenzene & 1 & & U & & UG/L & $06 / 09 / 2009$ & & HCJ-148-09 \\
\hline GWA05402VL & TRIP BLANK & NA & 1,2-Dichlorobenzene & 1 & & $\mathrm{u}$ & & UG/L & 06/09/2009 & & HCJ-148-09 \\
\hline GWA05301VL & FIELD BLANK & NA & Dichlorodifluoromethane & 1 & & $\mathrm{U}$ & & UG/L & $06 / 17 / 2009$ & & HCJ-153-09 \\
\hline GWA05301VL & FIELD BLANK & NA & Chloromethane & 1 & & U & & UG/L & $06 / 17 / 2009$ & & HCJ-153-09 \\
\hline GWA05301VL & FIELD BLANK & NA & Vinyl Chloride & 1 & & U & & UG/L & $06 / 17 / 2009$ & & HCJ-153-09 \\
\hline GWA05301VL & FIELD BLANK & NA & Bromomethane & 1 & & $\mathrm{u}$ & & UG/L & $06 / 17 / 2009$ & & HCJ-153-09 \\
\hline GWA05301VL & FIELD BLANK & NA & Chloroethane & 1 & & U & & UG/L & $06 / 17 / 2009$ & & HCJ-153-09 \\
\hline GWA05301VL & FIELD BLANK & NA & Trichlorofluoromethane & 1 & & U & & UG/L & $06 / 17 / 2009$ & & HCJ-153-09 \\
\hline GWA05301VL & FIELD BLANK & NA & Acetone & 5 & & $\mathrm{U}$ & & UG/L & $06 / 17 / 2009$ & & HCJ-153-09 \\
\hline GWA05301VL & FIELD BLANK & NA & 1,1-Dichloroethene & 1 & & $\mathrm{U}$ & & UG/L & $06 / 17 / 2009$ & & HCJ-153-09 \\
\hline GWA05301VL & FIELD BLANK & NA & Methyl acetate & 5 & & U & & UG/L & $06 / 17 / 2009$ & & HCJ-153-09 \\
\hline GWA05301VL & FIELD BLANK & NA & Methylene Chloride & 5 & & U & & UG/L & $06 / 17 / 2009$ & & HCJ-153-09 \\
\hline GWA05301VL & FIELD BLANK & NA & Carbon disulfide & 5 & & $\mathrm{U}$ & & UG/L & $06 / 17 / 2009$ & & HCJ-153-09 \\
\hline GWA05301VL & FIELD BLANK & NA & Tert-butyl methyl ether & 1 & & U & & UG/L & $06 / 17 / 2009$ & & HCJ-153-09 \\
\hline GWA05301VL & FIELD BLANK & NA & trans-1,2-Dichloroethylene & 1 & & $u$ & & UG/L & $06 / 17 / 2009$ & & HCJ-153-09 \\
\hline GWA05301VL & FIELD BLANK & NA & 1,1-Dichloroethane & 1 & & U & & UG/L & $06 / 17 / 2009$ & & HCJ-153-09 \\
\hline GWA05301VL & FIELD BLANK & NA & 2-Butanone & 5 & & $\mathrm{u}$ & & UG/L & $06 / 17 / 2009$ & & HCJ-153-09 \\
\hline GWA05301VL & FIELD BLANK & NA & cis-1,2-Dichloroethylene & 1 & & U & & UG/L & $06 / 17 / 2009$ & & HCJ-153-09 \\
\hline GWA05301VL & FIELD BLANK & NA & Chloroform & 0.368 & & $\mathrm{~J}$ & & UG/L & $06 / 17 / 2009$ & & HCJ-153-09 \\
\hline GWA05301VL & FIELD BLANK & NA & 1,1,1-Trichloroethane & 1 & & $\mathrm{u}$ & & UG/L & $06 / 17 / 2009$ & & HCJ-153-09 \\
\hline GWA05301VL & FIELD BLANK & NA & Cyclohexane & 1 & & $\mathrm{U}$ & & UG/L & $06 / 17 / 2009$ & & HCJ-153-09 \\
\hline GWA05301VL & FIELD BLANK & NA & Carbon tetrachloride & 1 & & U & & UG/L & $06 / 17 / 2009$ & & HCJ-153-09 \\
\hline GWA05301VL & FIELD BLANK & NA & 1,2-Dichloroethane & 1 & & $u$ & & UG/L & $06 / 17 / 2009$ & & HCJ-153-09 \\
\hline GWA05301VL & FIELD BLANK & NA & Benzene & 1 & & $\mathrm{U}$ & & UG/L & $06 / 17 / 2009$ & & HCJ-153-09 \\
\hline GWA05301VL & FIELD BLANK & NA & Trichloroethylene & 1 & & $\mathrm{U}$ & & UG/L & 06/17/2009 & & HCJ-153-09 \\
\hline GWA05301VL & FIELD BLANK & NA & 1,2-Dichloropropane & 1 & & $U$ & & UG/L & $06 / 17 / 2009$ & & HCJ-153-09 \\
\hline GWA05301VL & FIELD BLANK & NA & Methyl cyclohexane & 1 & & $\mathrm{u}$ & & UG/L & $06 / 17 / 2009$ & & HCJ-153-09 \\
\hline GWA05301VL & FIELD BLANK & NA & Bromodichloromethane & 1 & & U & & UG/L & $06 / 17 / 2009$ & & HCJ-153-09 \\
\hline GWA05301VL & FIELD BLANK & NA & 4-Methyl-2-pentanone & 5 & & U & & UG/L & $06 / 17 / 2009$ & & HCJ-153-09 \\
\hline GWA05301VL & FIELD BLANK & NA & cis-1,3-Dichloropropylene & 1 & & $u$ & & UG/L & $06 / 17 / 2009$ & & HCJ-153-09 \\
\hline GWA05301VL & FIELD BLANK & NA & Toluene & 1 & & $\mathrm{U}$ & & UG/L & $06 / 17 / 2009$ & & HCJ-153-09 \\
\hline GWA05301VL & FIELD BLANK & NA & trans-1,3-Dichloropropylene & 1 & & $\mathrm{U}$ & & UG/L & $06 / 17 / 2009$ & & HCJ-153-09 \\
\hline GWA05301VL & FIELD BLANK & NA & 1,1,2-Trichloroethane & 1 & & $U$ & & UG/L & 06/17/2009 & & HCJ-153-09 \\
\hline GWA05301VL & FIELD BLANK & NA & 2-Hexanone & 5 & & $\mathrm{U}$ & & UG/L & $06 / 17 / 2009$ & & HCJ-153-09 \\
\hline GWA05301VL & FIELD BLANK & NA & Tetrachloroethylene & 1 & & $\mathrm{U}$ & & UG/L & $06 / 17 / 2009$ & & HCJ-153-09 \\
\hline GWA05301VL & FIELD BLANK & NA & Dibromochloromethane & 1 & & $\mathrm{U}$ & & UG/L & $06 / 17 / 2009$ & & HCJ-153-09 \\
\hline
\end{tabular}


Table A-2. (continued).

\begin{tabular}{|c|c|c|c|c|c|c|c|c|c|c|c|}
\hline $\begin{array}{c}\text { Field Sample } \\
\text { Number }\end{array}$ & Location & Depth & Compound & $\begin{array}{c}\text { Sample } \\
\text { Result }\end{array}$ & $\begin{array}{l}\text { Sample } \\
\text { Error }\end{array}$ & $\begin{array}{c}\text { Result } \\
\text { Qualifier }\end{array}$ & $\begin{array}{l}\text { Validation } \\
\text { Flag }\end{array}$ & $\begin{array}{c}\text { Sample } \\
\text { Units }\end{array}$ & $\begin{array}{c}\text { Date Sample } \\
\text { Collected }\end{array}$ & MDA & L\&V Report Number \\
\hline GWA05301VL & FIELD BLANK & NA & 1,2-Dibromoethane & 1 & & $\mathrm{U}$ & & UG/L & $06 / 17 / 2009$ & & HCJ-153-09 \\
\hline GWA05301VL & FIELD BLANK & NA & Chlorobenzene & 1 & & $\mathrm{U}$ & & UG/L & 06/17/2009 & & HCJ-153-09 \\
\hline GWA05301VL & FIELD BLANK & NA & Ethylbenzene & 1 & & $\mathrm{U}$ & & UG/L & 06/17/2009 & & HCJ-153-09 \\
\hline GWA05301VL & FIELD BLANK & NA & Styrene & 1 & & $\mathrm{U}$ & & UG/L & 06/17/2009 & & HCJ-153-09 \\
\hline GWA05301VL & FIELD BLANK & NA & Bromoform & 1 & & $\mathrm{U}$ & & UG/L & 06/17/2009 & & HCJ-153-09 \\
\hline GWA05301VL & FIELD BLANK & NA & 1,1,2,2-Tetrachloroethane & 1 & & $\mathrm{U}$ & & UG/L & 06/17/2009 & & HCJ-153-09 \\
\hline GWA05301VL & FIELD BLANK & NA & Isopropylbenzene & 1 & & $\mathrm{U}$ & & UG/L & 06/17/2009 & & HCJ-153-09 \\
\hline GWA05301VL & FIELD BLANK & NA & 1,3-Dichlorobenzene & 1 & & $\mathrm{U}$ & & UG/L & 06/17/2009 & & HCJ-153-09 \\
\hline GWA05301VL & FIELD BLANK & NA & 1,4-Dichlorobenzene & 1 & & $\mathrm{U}$ & & UG/L & 06/17/2009 & & HCJ-153-09 \\
\hline GWA05301VL & FIELD BLANK & NA & 1,2-Dibromo-3-chloropropane & 1 & & $\mathrm{U}$ & & UG/L & 06/17/2009 & & HCJ-153-09 \\
\hline GWA05301VL & FIELD BLANK & NA & Trichlorotrifluoroethane & 5 & & $\mathrm{U}$ & & UG/L & $06 / 17 / 2009$ & & HCJ-153-09 \\
\hline GWA05301VL & FIELD BLANK & NA & Xylene (Total) & 1 & & $\mathrm{U}$ & & UG/L & 06/17/2009 & & HCJ-153-09 \\
\hline GWA05301VL & FIELD BLANK & NA & 1,2,4-Trichlorobenzene & 1 & & $\mathrm{U}$ & & UG/L & 06/17/2009 & & HCJ-153-09 \\
\hline GWA05301VL & FIELD BLANK & NA & 1,2-Dichlorobenzene & 1 & & $\mathrm{U}$ & & UG/L & 06/17/2009 & & HCJ-153-09 \\
\hline GWA05403VL & TRIP BLANK & NA & Dichlorodifluoromethane & 1 & & $\mathrm{U}$ & & UG/L & 06/15/2009 & & HCJ-153-09 \\
\hline GWA05403VL & TRIP BLANK & NA & Chloromethane & 1 & & $\mathrm{U}$ & & UG/L & 06/15/2009 & & HCJ-153-09 \\
\hline GWA05403VL & TRIP BLANK & NA & Vinyl Chloride & 1 & & $\mathrm{U}$ & & UG/L & 06/15/2009 & & HCJ-153-09 \\
\hline GWA05403VL & TRIP BLANK & NA & Bromomethane & 1 & & $\mathrm{U}$ & & UG/L & 06/15/2009 & & HCJ-153-09 \\
\hline GWA05403VL & TRIP BLANK & NA & Chloroethane & 1 & & $\mathrm{U}$ & & UG/L & 06/15/2009 & & HCJ-153-09 \\
\hline GWA05403VL & TRIP BLANK & NA & Trichlorofluoromethane & 1 & & $\mathrm{U}$ & & UG/L & 06/15/2009 & & HCJ-153-09 \\
\hline GWA05403VL & TRIP BLANK & NA & Acetone & 5 & & $\mathrm{U}$ & & UG/L & 06/15/2009 & & HCJ-153-09 \\
\hline GWA05403VL & TRIP BLANK & NA & 1,1-Dichloroethene & 1 & & $\mathrm{U}$ & & UG/L & 06/15/2009 & & HCJ-153-09 \\
\hline GWA05403VL & TRIP BLANK & NA & Methyl acetate & 5 & & $\mathrm{U}$ & & UG/L & $06 / 15 / 2009$ & & HCJ-153-09 \\
\hline GWA05403VL & TRIP BLANK & NA & Methylene Chloride & 5 & & $\mathrm{U}$ & & UG/L & 06/15/2009 & & HCJ-153-09 \\
\hline GWA05403VL & TRIP BLANK & NA & Carbon disulfide & 5 & & $\mathrm{U}$ & & UG/L & 06/15/2009 & & HCJ-153-09 \\
\hline GWA05403VL & TRIP BLANK & NA & Tert-butyl methyl ether & 1 & & $\mathrm{U}$ & & UG/L & 06/15/2009 & & HCJ-153-09 \\
\hline GWA05403VL & TRIP BLANK & NA & trans-1,2-Dichloroethylene & 1 & & U & & UG/L & 06/15/2009 & & HCJ-153-09 \\
\hline GWA05403VL & TRIP BLANK & NA & 1,1-Dichloroethane & 1 & & $\mathrm{U}$ & & UG/L & 06/15/2009 & & HCJ-153-09 \\
\hline GWA05403VL & TRIP BLANK & NA & 2-Butanone & 5 & & $\mathrm{U}$ & & UG/L & 06/15/2009 & & HCJ-153-09 \\
\hline GWA05403VL & TRIP BLANK & NA & cis-1,2-Dichloroethylene & 1 & & $\mathrm{U}$ & & UG/L & 06/15/2009 & & HCJ-153-09 \\
\hline GWA05403VL & TRIP BLANK & NA & Chloroform & 0.403 & & $\mathrm{~J}$ & & UG/L & 06/15/2009 & & HCJ-153-09 \\
\hline GWA05403VL & TRIP BLANK & NA & 1,1,1-Trichloroethane & 1 & & $\mathrm{U}$ & & UG/L & 06/15/2009 & & HCJ-153-09 \\
\hline GWA05403VL & TRIP BLANK & NA & Cyclohexane & 1 & & $\mathrm{U}$ & & UG/L & 06/15/2009 & & HCJ-153-09 \\
\hline GWA05403VL & TRIP BLANK & NA & Carbon tetrachloride & 1 & & $\mathrm{U}$ & & UG/L & 06/15/2009 & & HCJ-153-09 \\
\hline GWA05403VL & TRIP BLANK & NA & 1,2-Dichloroethane & 1 & & $\mathrm{U}$ & & UG/L & 06/15/2009 & & HCJ-153-09 \\
\hline GWA05403VL & TRIP BLANK & NA & Benzene & 1 & & $U$ & & UG/L & 06/15/2009 & & HCJ-153-09 \\
\hline GWA05403VL & TRIP BLANK & NA & Trichloroethylene & 1 & & $\mathrm{U}$ & & UG/L & 06/15/2009 & & HCJ-153-09 \\
\hline GWA05403VL & TRIP BLANK & NA & 1,2-Dichloropropane & 1 & & $\mathrm{U}$ & & UG/L & 06/15/2009 & & HCJ-153-09 \\
\hline GWA05403VL & TRIP BLANK & NA & Methyl cyclohexane & 1 & & $\mathrm{U}$ & & UG/L & $06 / 15 / 2009$ & & HCJ-153-09 \\
\hline GWA05403VL & TRIP BLANK & NA & Bromodichloromethane & 1 & & $\mathrm{U}$ & & UG/L & 06/15/2009 & & HCJ-153-09 \\
\hline GWA05403VL & TRIP BLANK & NA & 4-Methyl-2-pentanone & 5 & & $\mathrm{U}$ & & UG/L & 06/15/2009 & & HCJ-153-09 \\
\hline GWA05403VL & TRIP BLANK & NA & cis-1,3-Dichloropropylene & 1 & & $\mathrm{U}$ & & UG/L & 06/15/2009 & & HCJ-153-09 \\
\hline GWA05403VL & TRIP BLANK & NA & Toluene & 1 & & $\mathrm{U}$ & & UG/L & 06/15/2009 & & HCJ-153-09 \\
\hline GWA05403VL & TRIP BLANK & NA & trans-1,3-Dichloropropylene & 1 & & $\mathrm{U}$ & & UG/L & 06/15/2009 & & HCJ-153-09 \\
\hline
\end{tabular}


Table A-2. (continued).

\begin{tabular}{|c|c|c|c|c|c|c|c|c|c|c|c|}
\hline $\begin{array}{c}\text { Field Sample } \\
\text { Number }\end{array}$ & Location & Depth & Compound & $\begin{array}{l}\text { Sample } \\
\text { Result }\end{array}$ & $\begin{array}{c}\text { Sample } \\
\text { Error }\end{array}$ & $\begin{array}{c}\text { Result } \\
\text { Qualifier }\end{array}$ & $\begin{array}{c}\text { Validation } \\
\text { Flag }\end{array}$ & $\begin{array}{c}\text { Sample } \\
\text { Units }\end{array}$ & $\begin{array}{c}\text { Date Sample } \\
\text { Collected }\end{array}$ & MDA & L\&V Report Number \\
\hline GWA05403VL & TRIP BLANK & NA & 1,1,2-Trichloroethane & 1 & & $U$ & & UG/L & $06 / 15 / 2009$ & & HCJ-153-09 \\
\hline GWA05403VL & TRIP BLANK & NA & 2-Hexanone & 5 & & U & & UG/L & $06 / 15 / 2009$ & & HCJ-153-09 \\
\hline GWA05403VL & TRIP BLANK & NA & Tetrachloroethylene & 1 & & $\mathrm{U}$ & & UG/L & $06 / 15 / 2009$ & & HCJ-153-09 \\
\hline GWA05403VL & TRIP BLANK & NA & Dibromochloromethane & 1 & & $\mathrm{U}$ & & UG/L & $06 / 15 / 2009$ & & HCJ-153-09 \\
\hline GWA05403VL & TRIP BLANK & NA & 1,2-Dibromoethane & 1 & & U & & UG/L & $06 / 15 / 2009$ & & HCJ-153-09 \\
\hline GWA05403VL & TRIP BLANK & NA & Chlorobenzene & 1 & & U & & UG/L & $06 / 15 / 2009$ & & HCJ-153-09 \\
\hline GWA05403VL & TRIP BLANK & NA & Ethylbenzene & 1 & & $\mathrm{U}$ & & UG/L & $06 / 15 / 2009$ & & HCJ-153-09 \\
\hline GWA05403VL & TRIP BLANK & NA & Styrene & 1 & & U & & UG/L & $06 / 15 / 2009$ & & HCJ-153-09 \\
\hline GWA05403VL & TRIP BLANK & NA & Bromoform & 1 & & U & & UG/L & $06 / 15 / 2009$ & & HCJ-153-09 \\
\hline GWA05403VL & TRIP BLANK & NA & 1,1,2,2-Tetrachloroethane & 1 & & $\mathrm{U}$ & & UG/L & $06 / 15 / 2009$ & & HCJ-153-09 \\
\hline GWA05403VL & TRIP BLANK & NA & Isopropylbenzene & 1 & & $\mathrm{U}$ & & UG/L & $06 / 15 / 2009$ & & HCJ-153-09 \\
\hline GWA05403VL & TRIP BLANK & NA & 1,3-Dichlorobenzene & 1 & & U & & UG/L & $06 / 15 / 2009$ & & HCJ-153-09 \\
\hline GWA05403VL & TRIP BLANK & NA & 1,4-Dichlorobenzene & 1 & & U & & UG/L & $06 / 15 / 2009$ & & HCJ-153-09 \\
\hline GWA05403VL & TRIP BLANK & NA & 1,2-Dibromo-3-chloropropane & 1 & & $\mathrm{u}$ & & UG/L & $06 / 15 / 2009$ & & HCJ-153-09 \\
\hline GWA05403VL & TRIP BLANK & NA & Trichlorotrifluoroethane & 5 & & U & & UG/L & $06 / 15 / 2009$ & & HCJ-153-09 \\
\hline GWA05403VL & TRIP BLANK & NA & Xylene (Total) & 1 & & U & & UG/L & $06 / 15 / 2009$ & & HCJ-153-09 \\
\hline GWA05403VL & TRIP BLANK & NA & 1,2,4-Trichlorobenzene & 1 & & $\mathrm{u}$ & & UG/L & $06 / 15 / 2009$ & & HCJ-153-09 \\
\hline GWA05403VL & TRIP BLANK & NA & 1,2-Dichlorobenzene & 1 & & $\mathrm{u}$ & & UG/L & $06 / 15 / 2009$ & & HCJ-153-09 \\
\hline GWA05404VL & TRIP BLANK & NA & Dichlorodifluoromethane & 1 & & U & & UG/L & $06 / 22 / 2009$ & & HCJ-153-09 \\
\hline GWA05404VL & TRIP BLANK & NA & Chloromethane & 1 & & U & & UG/L & $06 / 22 / 2009$ & & HCJ-153-09 \\
\hline GWA05404VL & TRIP BLANK & NA & Vinyl Chloride & 1 & & $\mathrm{u}$ & & UG/L & $06 / 22 / 2009$ & & HCJ-153-09 \\
\hline GWA05404VL & TRIP BLANK & NA & Bromomethane & 1 & & U & & UG/L & $06 / 22 / 2009$ & & HCJ-153-09 \\
\hline GWA05404VL & TRIP BLANK & NA & Chloroethane & 1 & & U & & UG/L & $06 / 22 / 2009$ & & HCJ-153-09 \\
\hline GWA05404VL & TRIP BLANK & NA & Trichlorofluoromethane & 1 & & U & & UG/L & $06 / 22 / 2009$ & & HCJ-153-09 \\
\hline GWA05404VL & TRIP BLANK & NA & Acetone & 5 & & $\mathrm{U}$ & & UG/L & $06 / 22 / 2009$ & & HCJ-153-09 \\
\hline GWA05404VL & TRIP BLANK & NA & 1,1-Dichloroethene & 1 & & U & & UG/L & $06 / 22 / 2009$ & & HCJ-153-09 \\
\hline GWA05404VL & TRIP BLANK & NA & Methyl acetate & 5 & & $u$ & & UG/L & $06 / 22 / 2009$ & & HCJ-153-09 \\
\hline GWA05404VL & TRIP BLANK & NA & Methylene Chloride & 5 & & $\mathrm{u}$ & & UG/L & $06 / 22 / 2009$ & & HCJ-153-09 \\
\hline GWA05404VL & TRIP BLANK & NA & Carbon disulfide & 5 & & $\mathrm{U}$ & & UG/L & $06 / 22 / 2009$ & & HCJ-153-09 \\
\hline GWA05404VL & TRIP BLANK & NA & Tert-butyl methyl ether & 1 & & U & & UG/L & $06 / 22 / 2009$ & & HCJ-153-09 \\
\hline GWA05404VL & TRIP BLANK & NA & trans-1,2-Dichloroethylene & 1 & & $u$ & & UG/L & $06 / 22 / 2009$ & & HCJ-153-09 \\
\hline GWA05404VL & TRIP BLANK & NA & 1,1-Dichloroethane & 1 & & $\mathrm{u}$ & & UG/L & $06 / 22 / 2009$ & & HCJ-153-09 \\
\hline GWA05404VL & TRIP BLANK & NA & 2-Butanone & 5 & & U & & UG/L & $06 / 22 / 2009$ & & HCJ-153-09 \\
\hline GWA05404VL & TRIP BLANK & NA & cis-1,2-Dichloroethylene & 1 & & $U$ & & UG/L & $06 / 22 / 2009$ & & HCJ-153-09 \\
\hline GWA05404VL & TRIP BLANK & NA & Chloroform & 1 & & $\mathrm{u}$ & & UG/L & $06 / 22 / 2009$ & & HCJ-153-09 \\
\hline GWA05404VL & TRIP BLANK & NA & 1,1,1-Trichloroethane & 1 & & $\mathrm{U}$ & & UG/L & $06 / 22 / 2009$ & & HCJ-153-09 \\
\hline GWA05404VL & TRIP BLANK & NA & Cyclohexane & 1 & & $u$ & & UG/L & $06 / 22 / 2009$ & & HCJ-153-09 \\
\hline GWA05404VL & TRIP BLANK & NA & Carbon tetrachloride & 1 & & $u$ & & UG/L & $06 / 22 / 2009$ & & HCJ-153-09 \\
\hline GWA05404VL & TRIP BLANK & NA & 1,2-Dichloroethane & 1 & & $\mathrm{U}$ & & UG/L & $06 / 22 / 2009$ & & HCJ-153-09 \\
\hline GWA05404VL & TRIP BLANK & NA & Benzene & 1 & & $\mathrm{U}$ & & UG/L & $06 / 22 / 2009$ & & HCJ-153-09 \\
\hline GWA05404VL & TRIP BLANK & NA & Trichloroethylene & 1 & & $U$ & & UG/L & $06 / 22 / 2009$ & & HCJ-153-09 \\
\hline GWA05404VL & TRIP BLANK & NA & 1,2-Dichloropropane & 1 & & $\mathrm{U}$ & & UG/L & $06 / 22 / 2009$ & & HCJ-153-09 \\
\hline GWA05404VL & TRIP BLANK & NA & Methyl cyclohexane & 1 & & $\mathrm{U}$ & & UG/L & $06 / 22 / 2009$ & & HCJ-153-09 \\
\hline GWA05404VL & TRIP BLANK & NA & Bromodichloromethane & 1 & & U & & UG/L & $06 / 22 / 2009$ & & HCJ-153-09 \\
\hline
\end{tabular}


Table A-2. (continued).

\begin{tabular}{|c|c|c|c|c|c|c|c|c|c|c|c|}
\hline $\begin{array}{c}\text { Field Sample } \\
\text { Number }\end{array}$ & Location & Depth & Compound & $\begin{array}{c}\text { Sample } \\
\text { Result }\end{array}$ & $\begin{array}{c}\text { Sample } \\
\text { Error }\end{array}$ & $\begin{array}{c}\text { Result } \\
\text { Qualifier }\end{array}$ & $\begin{array}{c}\text { Validation } \\
\text { Flag }\end{array}$ & $\begin{array}{c}\text { Sample } \\
\text { Units }\end{array}$ & $\begin{array}{c}\text { Date Sample } \\
\text { Collected }\end{array}$ & MDA & L\&V Report Number \\
\hline GWA05404VL & TRIP BLANK & NA & 4-Methyl-2-pentanone & 5 & & $U$ & & UG/L & $06 / 22 / 2009$ & & HCJ-153-09 \\
\hline GWA05404VL & TRIP BLANK & NA & cis-1,3-Dichloropropylene & 1 & & U & & UG/L & $06 / 22 / 2009$ & & HCJ-153-09 \\
\hline GWA05404VL & TRIP BLANK & NA & Toluene & 1 & & $\mathrm{u}$ & & UG/L & $06 / 22 / 2009$ & & HCJ-153-09 \\
\hline GWA05404VL & TRIP BLANK & NA & trans-1,3-Dichloropropylene & 1 & & $\mathrm{U}$ & & UG/L & $06 / 22 / 2009$ & & HCJ-153-09 \\
\hline GWA05404VL & TRIP BLANK & NA & 1,1,2-Trichloroethane & 1 & & U & & UG/L & $06 / 22 / 2009$ & & HCJ-153-09 \\
\hline GWA05404VL & TRIP BLANK & NA & 2-Hexanone & 5 & & U & & UG/L & $06 / 22 / 2009$ & & HCJ-153-09 \\
\hline GWA05404VL & TRIP BLANK & NA & Tetrachloroethylene & 1 & & $\mathrm{U}$ & & UG/L & $06 / 22 / 2009$ & & HCJ-153-09 \\
\hline GWA05404VL & TRIP BLANK & NA & Dibromochloromethane & 1 & & U & & UG/L & $06 / 22 / 2009$ & & HCJ-153-09 \\
\hline GWA05404VL & TRIP BLANK & NA & 1,2-Dibromoethane & 1 & & U & & UG/L & $06 / 22 / 2009$ & & HCJ-153-09 \\
\hline GWA05404VL & TRIP BLANK & NA & Chlorobenzene & 1 & & $\mathrm{u}$ & & UG/L & $06 / 22 / 2009$ & & HCJ-153-09 \\
\hline GWA05404VL & TRIP BLANK & NA & Ethylbenzene & 1 & & $\mathrm{u}$ & & UG/L & $06 / 22 / 2009$ & & HCJ-153-09 \\
\hline GWA05404VL & TRIP BLANK & NA & Styrene & 1 & & U & & UG/L & $06 / 22 / 2009$ & & HCJ-153-09 \\
\hline GWA05404VL & TRIP BLANK & NA & Bromoform & 1 & & U & & UG/L & $06 / 22 / 2009$ & & HCJ-153-09 \\
\hline GWA05404VL & TRIP BLANK & NA & 1,1,2,2-Tetrachloroethane & 1 & & $\mathrm{u}$ & & UG/L & $06 / 22 / 2009$ & & HCJ-153-09 \\
\hline GWA05404VL & TRIP BLANK & NA & Isopropylbenzene & 1 & & U & & UG/L & $06 / 22 / 2009$ & & HCJ-153-09 \\
\hline GWA05404VL & TRIP BLANK & NA & 1,3-Dichlorobenzene & 1 & & U & & UG/L & $06 / 22 / 2009$ & & HCJ-153-09 \\
\hline GWA05404VL & TRIP BLANK & NA & 1,4-Dichlorobenzene & 1 & & $u$ & & UG/L & $06 / 22 / 2009$ & & HCJ-153-09 \\
\hline GWA05404VL & TRIP BLANK & NA & 1,2-Dibromo-3-chloropropane & 1 & & $\mathrm{u}$ & & UG/L & $06 / 22 / 2009$ & & HCJ-153-09 \\
\hline GWA05404VL & TRIP BLANK & NA & Trichlorotrifluoroethane & 5 & & U & & UG/L & $06 / 22 / 2009$ & & HCJ-153-09 \\
\hline GWA05404VL & TRIP BLANK & NA & Xylene (Total) & 1 & & U & & UG/L & $06 / 22 / 2009$ & & HCJ-153-09 \\
\hline GWA05404VL & TRIP BLANK & NA & 1,2,4-Trichlorobenzene & 1 & & $\mathrm{U}$ & & UG/L & $06 / 22 / 2009$ & & HCJ-153-09 \\
\hline GWA05404VL & TRIP BLANK & NA & 1,2-Dichlorobenzene & 1 & & U & & UG/L & $06 / 22 / 2009$ & & HCJ-153-09 \\
\hline GWA05404VL & TRIP BLANK & NA & Unknown & 5.4 & & $\mathrm{~J}$ & & UG/L & $06 / 22 / 2009$ & & \\
\hline GWA05404VL & TRIP BLANK & NA & Unknown Siloxane & 13.5 & & $\mathrm{~J}$ & & UG/L & $06 / 22 / 2009$ & & \\
\hline GWA05302VL & FIELD BLANK & NA & Dichlorodifluoromethane & 1 & & $\mathrm{U}$ & & UG/L & $07 / 02 / 2009$ & & HCJ-166-09 \\
\hline GWA05302VL & FIELD BLANK & NA & Chloromethane & 1 & & U & & UG/L & $07 / 02 / 2009$ & & HCJ-166-09 \\
\hline GWA05302VL & FIELD BLANK & NA & Vinyl Chloride & 1 & & $u$ & & UG/L & $07 / 02 / 2009$ & & HCJ-166-09 \\
\hline GWA05302VL & FIELD BLANK & NA & Bromomethane & 1 & & $\mathrm{u}$ & & UG/L & $07 / 02 / 2009$ & & HCJ-166-09 \\
\hline GWA05302VL & FIELD BLANK & NA & Chloroethane & 1 & & $\mathrm{U}$ & & UG/L & $07 / 02 / 2009$ & & HCJ-166-09 \\
\hline GWA05302VL & FIELD BLANK & NA & Trichlorofluoromethane & 1 & & U & & UG/L & $07 / 02 / 2009$ & & HCJ-166-09 \\
\hline GWA05302VL & FIELD BLANK & NA & Acetone & 5 & & $u$ & & UG/L & $07 / 02 / 2009$ & & HCJ-166-09 \\
\hline GWA05302VL & FIELD BLANK & NA & 1,1-Dichloroethene & 1 & & $\mathrm{u}$ & & UG/L & $07 / 02 / 2009$ & & HCJ-166-09 \\
\hline GWA05302VL & FIELD BLANK & NA & Methyl acetate & 5 & & $\mathrm{U}$ & & UG/L & $07 / 02 / 2009$ & & HCJ-166-09 \\
\hline GWA05302VL & FIELD BLANK & NA & Methylene Chloride & 5 & & $U$ & & UG/L & $07 / 02 / 2009$ & & HCJ-166-09 \\
\hline GWA05302VL & FIELD BLANK & NA & Carbon disulfide & 5 & & $\mathrm{u}$ & & UG/L & $07 / 02 / 2009$ & & HCJ-166-09 \\
\hline GWA05302VL & FIELD BLANK & NA & Tert-butyl methyl ether & 1 & & $\mathrm{U}$ & & UG/L & $07 / 02 / 2009$ & & HCJ-166-09 \\
\hline GWA05302VL & FIELD BLANK & NA & trans-1,2-Dichloroethylene & 1 & & U & & UG/L & $07 / 02 / 2009$ & & HCJ-166-09 \\
\hline GWA05302VL & FIELD BLANK & NA & 1,1-Dichloroethane & 1 & & $u$ & & UG/L & $07 / 02 / 2009$ & & HCJ-166-09 \\
\hline GWA05302VL & FIELD BLANK & NA & 2-Butanone & 5 & & $\mathrm{U}$ & & UG/L & $07 / 02 / 2009$ & & HCJ-166-09 \\
\hline GWA05302VL & FIELD BLANK & NA & cis-1,2-Dichloroethylene & 1 & & $\mathrm{U}$ & & UG/L & $07 / 02 / 2009$ & & HCJ-166-09 \\
\hline GWA05302VL & FIELD BLANK & NA & Chloroform & 2.8 & & & $\mathrm{~J}$ & UG/L & $07 / 02 / 2009$ & & HCJ-166-09 \\
\hline GWA05302VL & FIELD BLANK & NA & 1,1,1-Trichloroethane & 1 & & $\mathrm{U}$ & & UG/L & $07 / 02 / 2009$ & & HCJ-166-09 \\
\hline GWA05302VL & FIELD BLANK & NA & Cyclohexane & 1 & & $\mathrm{U}$ & & UG/L & $07 / 02 / 2009$ & & HCJ-166-09 \\
\hline GWA05302VL & FIELD BLANK & NA & Carbon tetrachloride & 1 & & $U$ & & UG/L & $07 / 02 / 2009$ & & HCJ-166-09 \\
\hline
\end{tabular}


Table A-2. (continued).

\begin{tabular}{|c|c|c|c|c|c|c|c|c|c|c|c|}
\hline $\begin{array}{c}\text { Field Sample } \\
\text { Number }\end{array}$ & Location & Depth & Compound & $\begin{array}{c}\text { Sample } \\
\text { Result }\end{array}$ & $\begin{array}{l}\text { Sample } \\
\text { Error }\end{array}$ & $\begin{array}{c}\text { Result } \\
\text { Qualifier }\end{array}$ & $\begin{array}{l}\text { Validation } \\
\text { Flag }\end{array}$ & $\begin{array}{c}\text { Sample } \\
\text { Units }\end{array}$ & $\begin{array}{c}\text { Date Sample } \\
\text { Collected }\end{array}$ & MDA & L\&V Report Number \\
\hline GWA05302VL & FIELD BLANK & NA & 1,2-Dichloroethane & 1 & & $\mathrm{U}$ & & UG/L & $07 / 02 / 2009$ & & HCJ-166-09 \\
\hline GWA05302VL & FIELD BLANK & NA & Benzene & 1 & & $\mathrm{U}$ & & UG/L & 07/02/2009 & & HCJ-166-09 \\
\hline GWA05302VL & FIELD BLANK & NA & Trichloroethylene & 1 & & $\mathrm{U}$ & & UG/L & 07/02/2009 & & HCJ-166-09 \\
\hline GWA05302VL & FIELD BLANK & NA & 1,2-Dichloropropane & 1 & & $\mathrm{U}$ & & UG/L & 07/02/2009 & & HCJ-166-09 \\
\hline GWA05302VL & FIELD BLANK & NA & Methyl cyclohexane & 1 & & $\mathrm{U}$ & & UG/L & 07/02/2009 & & HCJ-166-09 \\
\hline GWA05302VL & FIELD BLANK & NA & Bromodichloromethane & 1 & & $\mathrm{U}$ & & UG/L & 07/02/2009 & & HCJ-166-09 \\
\hline GWA05302VL & FIELD BLANK & NA & 4-Methyl-2-pentanone & 5 & & U & & UG/L & 07/02/2009 & & HCJ-166-09 \\
\hline GWA05302VL & FIELD BLANK & NA & cis-1,3-Dichloropropylene & 1 & & $\mathrm{U}$ & & UG/L & 07/02/2009 & & HCJ-166-09 \\
\hline GWA05302VL & FIELD BLANK & NA & Toluene & 1 & & $\mathrm{U}$ & & UG/L & 07/02/2009 & & HCJ-166-09 \\
\hline GWA05302VL & FIELD BLANK & NA & trans-1,3-Dichloropropylene & 1 & & $\mathrm{U}$ & & UG/L & 07/02/2009 & & HCJ-166-09 \\
\hline GWA05302VL & FIELD BLANK & NA & 1,1,2-Trichloroethane & 1 & & $\mathrm{U}$ & & UG/L & 07/02/2009 & & HCJ-166-09 \\
\hline GWA05302VL & FIELD BLANK & NA & 2-Hexanone & 5 & & $\mathrm{U}$ & & UG/L & 07/02/2009 & & HCJ-166-09 \\
\hline GWA05302VL & FIELD BLANK & NA & Tetrachloroethylene & 0.336 & & BJ & $\mathrm{J}$ & UG/L & $07 / 02 / 2009$ & & HCJ-166-09 \\
\hline GWA05302VL & FIELD BLANK & NA & Dibromochloromethane & 1 & & $\mathrm{U}$ & & UG/L & 07/02/2009 & & HCJ-166-09 \\
\hline GWA05302VL & FIELD BLANK & NA & 1,2-Dibromoethane & 1 & & $\mathrm{U}$ & & UG/L & 07/02/2009 & & HCJ-166-09 \\
\hline GWA05302VL & FIELD BLANK & NA & Chlorobenzene & 1 & & $\mathrm{U}$ & & UG/L & $07 / 02 / 2009$ & & HCJ-166-09 \\
\hline GWA05302VL & FIELD BLANK & NA & Ethylbenzene & 1 & & $\mathrm{U}$ & & UG/L & 07/02/2009 & & HCJ-166-09 \\
\hline GWA05302VL & FIELD BLANK & NA & Styrene & 1 & & $\mathrm{U}$ & & UG/L & 07/02/2009 & & HCJ-166-09 \\
\hline GWA05302VL & FIELD BLANK & NA & Bromoform & 1 & & $\mathrm{U}$ & & UG/L & 07/02/2009 & & HCJ-166-09 \\
\hline GWA05302VL & FIELD BLANK & NA & 1,1,2,2-Tetrachloroethane & 1 & & $\mathrm{U}$ & & UG/L & 07/02/2009 & & HCJ-166-09 \\
\hline GWA05302VL & FIELD BLANK & NA & Isopropylbenzene & 1 & & $\mathrm{U}$ & & UG/L & 07/02/2009 & & HCJ-166-09 \\
\hline GWA05302VL & FIELD BLANK & NA & 1,3-Dichlorobenzene & 1 & & $\mathrm{U}$ & & UG/L & 07/02/2009 & & HCJ-166-09 \\
\hline GWA05302VL & FIELD BLANK & NA & 1,4-Dichlorobenzene & 1 & & $\mathrm{U}$ & & UG/L & $07 / 02 / 2009$ & & HCJ-166-09 \\
\hline GWA05302VL & FIELD BLANK & NA & 1,2-Dibromo-3-chloropropane & 1 & & $\mathrm{U}$ & & UG/L & 07/02/2009 & & HCJ-166-09 \\
\hline GWA05302VL & FIELD BLANK & NA & Trichlorotrifluoroethane & 5 & & $\mathrm{U}$ & & UG/L & 07/02/2009 & & HCJ-166-09 \\
\hline GWA05302VL & FIELD BLANK & NA & Xylene (Total) & 1 & & $\mathrm{U}$ & & UG/L & 07/02/2009 & & HCJ-166-09 \\
\hline GWA05302VL & FIELD BLANK & NA & 1,2,4-Trichlorobenzene & 1 & & U & & UG/L & 07/02/2009 & & HCJ-166-09 \\
\hline GWA05302VL & FIELD BLANK & NA & 1,2-Dichlorobenzene & 1 & & $\mathrm{U}$ & & UG/L & 07/02/2009 & & HCJ-166-09 \\
\hline GWA05302VL & FIELD BLANK & NA & Unknown & 11.3 & & $\mathrm{~J}$ & & UG/L & 07/02/2009 & & \\
\hline GWA05405VL & TRIP BLANK & NA & Dichlorodifluoromethane & 1 & & $\mathrm{U}$ & & UG/L & 06/29/2009 & & HCJ-166-09 \\
\hline GWA05405VL & TRIP BLANK & NA & Chloromethane & 1 & & $U$ & & UG/L & 06/29/2009 & & HCJ-166-09 \\
\hline GWA05405VL & TRIP BLANK & NA & Vinyl Chloride & 1 & & $\mathrm{U}$ & & UG/L & 06/29/2009 & & HCJ-166-09 \\
\hline GWA05405VL & TRIP BLANK & NA & Bromomethane & 1 & & $\mathrm{U}$ & & UG/L & 06/29/2009 & & HCJ-166-09 \\
\hline GWA05405VL & TRIP BLANK & NA & Chloroethane & 1 & & $\mathrm{U}$ & & UG/L & 06/29/2009 & & HCJ-166-09 \\
\hline GWA05405VL & TRIP BLANK & NA & Trichlorofluoromethane & 1 & & $\mathrm{U}$ & & UG/L & 06/29/2009 & & HCJ-166-09 \\
\hline GWA05405VL & TRIP BLANK & NA & Acetone & 5 & & $\mathrm{U}$ & & UG/L & 06/29/2009 & & HCJ-166-09 \\
\hline GWA05405VL & TRIP BLANK & NA & 1,1-Dichloroethene & 1 & & $\mathrm{U}$ & & UG/L & 06/29/2009 & & HCJ-166-09 \\
\hline GWA05405VL & TRIP BLANK & NA & Methyl acetate & 5 & & $\mathrm{U}$ & & UG/L & 06/29/2009 & & HCJ-166-09 \\
\hline GWA05405VL & TRIP BLANK & NA & Methylene Chloride & 5 & & $\mathrm{U}$ & & UG/L & 06/29/2009 & & HCJ-166-09 \\
\hline GWA05405VL & TRIP BLANK & NA & Carbon disulfide & 5 & & $\mathrm{U}$ & & UG/L & 06/29/2009 & & HCJ-166-09 \\
\hline GWA05405VL & TRIP BLANK & NA & Tert-butyl methyl ether & 1 & & $\mathrm{U}$ & & UG/L & 06/29/2009 & & HCJ-166-09 \\
\hline GWA05405VL & TRIP BLANK & NA & trans-1,2-Dichloroethylene & 1 & & $\mathrm{U}$ & & UG/L & 06/29/2009 & & HCJ-166-09 \\
\hline GWA05405VL & TRIP BLANK & NA & 1,1-Dichloroethane & 1 & & U & & UG/L & 06/29/2009 & & HCJ-166-09 \\
\hline GWA05405VL & TRIP BLANK & NA & 2-Butanone & 5 & & $\mathrm{U}$ & & UG/L & 06/29/2009 & & HCJ-166-09 \\
\hline
\end{tabular}


Table A-2. (continued).

\begin{tabular}{|c|c|c|c|c|c|c|c|c|c|c|c|}
\hline $\begin{array}{c}\text { Field Sample } \\
\text { Number }\end{array}$ & Location & Depth & Compound & $\begin{array}{c}\text { Sample } \\
\text { Result }\end{array}$ & $\begin{array}{c}\text { Sample } \\
\text { Error }\end{array}$ & $\begin{array}{c}\text { Result } \\
\text { Qualifier }\end{array}$ & $\begin{array}{c}\text { Validation } \\
\text { Flag }\end{array}$ & $\begin{array}{c}\text { Sample } \\
\text { Units }\end{array}$ & $\begin{array}{c}\text { Date Sample } \\
\text { Collected }\end{array}$ & MDA & L\&V Report Number \\
\hline GWA05405VL & TRIP BLANK & NA & cis-1,2-Dichloroethylene & 1 & & $U$ & & UG/L & $06 / 29 / 2009$ & & HCJ-166-09 \\
\hline GWA05405VL & TRIP BLANK & NA & Chloroform & 1 & & U & & UG/L & $06 / 29 / 2009$ & & HCJ-166-09 \\
\hline GWA05405VL & TRIP BLANK & NA & 1,1,1-Trichloroethane & 1 & & $\mathrm{U}$ & & UG/L & $06 / 29 / 2009$ & & HCJ-166-09 \\
\hline GWA05405VL & TRIP BLANK & NA & Cyclohexane & 1 & & $\mathrm{U}$ & & UG/L & $06 / 29 / 2009$ & & HCJ-166-09 \\
\hline GWA05405VL & TRIP BLANK & NA & Carbon tetrachloride & 1 & & U & & UG/L & $06 / 29 / 2009$ & & HCJ-166-09 \\
\hline GWA05405VL & TRIP BLANK & NA & 1,2-Dichloroethane & 1 & & U & & UG/L & $06 / 29 / 2009$ & & HCJ-166-09 \\
\hline GWA05405VL & TRIP BLANK & NA & Benzene & 1 & & $\mathrm{U}$ & & UG/L & $06 / 29 / 2009$ & & HCJ-166-09 \\
\hline GWA05405VL & TRIP BLANK & NA & Trichloroethylene & 1 & & U & & UG/L & $06 / 29 / 2009$ & & HCJ-166-09 \\
\hline GWA05405VL & TRIP BLANK & NA & 1,2-Dichloropropane & 1 & & U & & UG/L & $06 / 29 / 2009$ & & HCJ-166-09 \\
\hline GWA05405VL & TRIP BLANK & NA & Methyl cyclohexane & 1 & & $\mathrm{U}$ & & UG/L & $06 / 29 / 2009$ & & HCJ-166-09 \\
\hline GWA05405VL & TRIP BLANK & NA & Bromodichloromethane & 1 & & $\mathrm{U}$ & & UG/L & $06 / 29 / 2009$ & & HCJ-166-09 \\
\hline GWA05405VL & TRIP BLANK & NA & 4-Methyl-2-pentanone & 5 & & U & & UG/L & $06 / 29 / 2009$ & & HCJ-166-09 \\
\hline GWA05405VL & TRIP BLANK & NA & cis-1,3-Dichloropropylene & 1 & & U & & UG/L & $06 / 29 / 2009$ & & HCJ-166-09 \\
\hline GWA05405VL & TRIP BLANK & NA & Toluene & 1 & & $\mathrm{u}$ & & UG/L & $06 / 29 / 2009$ & & HCJ-166-09 \\
\hline GWA05405VL & TRIP BLANK & NA & trans-1,3-Dichloropropylene & 1 & & U & & UG/L & $06 / 29 / 2009$ & & HCJ-166-09 \\
\hline GWA05405VL & TRIP BLANK & NA & 1,1,2-Trichloroethane & 1 & & U & & UG/L & $06 / 29 / 2009$ & & HCJ-166-09 \\
\hline GWA05405VL & TRIP BLANK & NA & 2-Hexanone & 5 & & $\mathrm{U}$ & & UG/L & $06 / 29 / 2009$ & & HCJ-166-09 \\
\hline GWA05405VL & TRIP BLANK & NA & Tetrachloroethylene & 0.349 & & BJ & $\mathrm{J}$ & UG/L & $06 / 29 / 2009$ & & HCJ-166-09 \\
\hline GWA05405VL & TRIP BLANK & NA & Dibromochloromethane & 1 & & $\mathrm{U}$ & & UG/L & $06 / 29 / 2009$ & & HCJ-166-09 \\
\hline GWA05405VL & TRIP BLANK & NA & 1,2-Dibromoethane & 1 & & U & & UG/L & $06 / 29 / 2009$ & & HCJ-166-09 \\
\hline GWA05405VL & TRIP BLANK & NA & Chlorobenzene & 1 & & $\mathrm{u}$ & & UG/L & $06 / 29 / 2009$ & & HCJ-166-09 \\
\hline GWA05405VL & TRIP BLANK & NA & Ethylbenzene & 1 & & U & & UG/L & $06 / 29 / 2009$ & & HCJ-166-09 \\
\hline GWA05405VL & TRIP BLANK & NA & Styrene & 1 & & U & & UG/L & $06 / 29 / 2009$ & & HCJ-166-09 \\
\hline GWA05405VL & TRIP BLANK & NA & Bromoform & 1 & & U & & UG/L & $06 / 29 / 2009$ & & HCJ-166-09 \\
\hline GWA05405VL & TRIP BLANK & NA & 1,1,2,2-Tetrachloroethane & 1 & & $\mathrm{u}$ & & UG/L & $06 / 29 / 2009$ & & HCJ-166-09 \\
\hline GWA05405VL & TRIP BLANK & NA & Isopropylbenzene & 1 & & U & & UG/L & $06 / 29 / 2009$ & & HCJ-166-09 \\
\hline GWA05405VL & TRIP BLANK & NA & 1,3-Dichlorobenzene & 1 & & $u$ & & UG/L & $06 / 29 / 2009$ & & HCJ-166-09 \\
\hline GWA05405VL & TRIP BLANK & NA & 1,4-Dichlorobenzene & 1 & & $\mathrm{u}$ & & UG/L & $06 / 29 / 2009$ & & HCJ-166-09 \\
\hline GWA05405VL & TRIP BLANK & NA & 1,2-Dibromo-3-chloropropane & 1 & & $\mathrm{U}$ & & UG/L & $06 / 29 / 2009$ & & HCJ-166-09 \\
\hline GWA05405VL & TRIP BLANK & NA & Trichlorotrifluoroethane & 5 & & $\mathrm{U}$ & & UG/L & $06 / 29 / 2009$ & & HCJ-166-09 \\
\hline GWA05405VL & TRIP BLANK & NA & Xylene (Total) & 1 & & $u$ & & UG/L & $06 / 29 / 2009$ & & HCJ-166-09 \\
\hline GWA05405VL & TRIP BLANK & NA & 1,2,4-Trichlorobenzene & 1 & & $\mathrm{u}$ & & UG/L & $06 / 29 / 2009$ & & HCJ-166-09 \\
\hline GWA05405VL & TRIP BLANK & NA & 1,2-Dichlorobenzene & 1 & & $\mathrm{U}$ & & UG/L & $06 / 29 / 2009$ & & HCJ-166-09 \\
\hline GWA05405VL & TRIP BLANK & NA & Unknown & 10.5 & & $\mathrm{~J}$ & & UG/L & $06 / 29 / 2009$ & & \\
\hline GWA05406VL & TRIP BLANK & NA & Dichlorodifluoromethane & 1 & & $\mathrm{u}$ & & UG/L & $07 / 07 / 2009$ & & HCJ-166-09 \\
\hline GWA05406VL & TRIP BLANK & NA & Chloromethane & 1 & & $\mathrm{U}$ & & UG/L & 07/07/2009 & & HCJ-166-09 \\
\hline GWA05406VL & TRIP BLANK & NA & Vinyl Chloride & 1 & & U & & UG/L & 07/07/2009 & & HCJ-166-09 \\
\hline GWA05406VL & TRIP BLANK & NA & Bromomethane & 1 & & $u$ & & UG/L & $07 / 07 / 2009$ & & HCJ-166-09 \\
\hline GWA05406VL & TRIP BLANK & NA & Chloroethane & 1 & & $\mathrm{U}$ & & UG/L & 07/07/2009 & & HCJ-166-09 \\
\hline GWA05406VL & TRIP BLANK & NA & Trichlorofluoromethane & 1 & & $\mathrm{U}$ & & UG/L & $07 / 07 / 2009$ & & HCJ-166-09 \\
\hline GWA05406VL & TRIP BLANK & NA & Acetone & 5 & & $U$ & & UG/L & 07/07/2009 & & HCJ-166-09 \\
\hline GWA05406VL & TRIP BLANK & NA & 1,1-Dichloroethene & 1 & & $\mathrm{U}$ & & UG/L & $07 / 07 / 2009$ & & HCJ-166-09 \\
\hline GWA05406VL & TRIP BLANK & NA & Methyl acetate & 5 & & $\mathrm{U}$ & & UG/L & 07/07/2009 & & HCJ-166-09 \\
\hline GWA05406VL & TRIP BLANK & NA & Methylene Chloride & 5 & & U & & UG/L & 07/07/2009 & & HCJ-166-09 \\
\hline
\end{tabular}


Table A-2. (continued).

\begin{tabular}{|c|c|c|c|c|c|c|c|c|c|c|c|}
\hline $\begin{array}{c}\text { Field Sample } \\
\text { Number }\end{array}$ & Location & Depth & Compound & $\begin{array}{c}\text { Sample } \\
\text { Result }\end{array}$ & $\begin{array}{c}\text { Sample } \\
\text { Error }\end{array}$ & $\begin{array}{c}\text { Result } \\
\text { Qualifier }\end{array}$ & $\begin{array}{c}\text { Validation } \\
\text { Flag }\end{array}$ & $\begin{array}{c}\text { Sample } \\
\text { Units }\end{array}$ & $\begin{array}{c}\text { Date Sample } \\
\text { Collected }\end{array}$ & MDA & L\&V Report Number \\
\hline GWA05406VL & TRIP BLANK & NA & Carbon disulfide & 5 & & $U$ & & UG/L & $07 / 07 / 2009$ & & HCJ-166-09 \\
\hline GWA05406VL & TRIP BLANK & NA & Tert-butyl methyl ether & 1 & & U & & UG/L & $07 / 07 / 2009$ & & HCJ-166-09 \\
\hline GWA05406VL & TRIP BLANK & NA & trans-1,2-Dichloroethylene & 1 & & $\mathrm{U}$ & & UG/L & $07 / 07 / 2009$ & & HCJ-166-09 \\
\hline GWA05406VL & TRIP BLANK & NA & 1,1-Dichloroethane & 1 & & $\mathrm{U}$ & & UG/L & 07/07/2009 & & HCJ-166-09 \\
\hline GWA05406VL & TRIP BLANK & NA & 2-Butanone & 5 & & U & & UG/L & $07 / 07 / 2009$ & & HCJ-166-09 \\
\hline GWA05406VL & TRIP BLANK & NA & cis-1,2-Dichloroethylene & 1 & & U & & UG/L & $07 / 07 / 2009$ & & HCJ-166-09 \\
\hline GWA05406VL & TRIP BLANK & NA & Chloroform & 3.18 & & & $\mathrm{~J}$ & UG/L & $07 / 07 / 2009$ & & HCJ-166-09 \\
\hline GWA05406VL & TRIP BLANK & NA & 1,1,1-Trichloroethane & 1 & & U & & UG/L & $07 / 07 / 2009$ & & HCJ-166-09 \\
\hline GWA05406VL & TRIP BLANK & NA & Cyclohexane & 1 & & U & & UG/L & $07 / 07 / 2009$ & & HCJ-166-09 \\
\hline GWA05406VL & TRIP BLANK & NA & Carbon tetrachloride & 1 & & $\mathrm{U}$ & & UG/L & 07/07/2009 & & HCJ-166-09 \\
\hline GWA05406VL & TRIP BLANK & NA & 1,2-Dichloroethane & 1 & & $\mathrm{U}$ & & UG/L & $07 / 07 / 2009$ & & HCJ-166-09 \\
\hline GWA05406VL & TRIP BLANK & NA & Benzene & 1 & & U & & UG/L & $07 / 07 / 2009$ & & HCJ-166-09 \\
\hline GWA05406VL & TRIP BLANK & NA & Trichloroethylene & 1 & & U & & UG/L & $07 / 07 / 2009$ & & HCJ-166-09 \\
\hline GWA05406VL & TRIP BLANK & NA & 1,2-Dichloropropane & 1 & & $\mathrm{u}$ & & UG/L & $07 / 07 / 2009$ & & HCJ-166-09 \\
\hline GWA05406VL & TRIP BLANK & NA & Methyl cyclohexane & 1 & & U & & UG/L & $07 / 07 / 2009$ & & HCJ-166-09 \\
\hline GWA05406VL & TRIP BLANK & NA & Bromodichloromethane & 1 & & U & & UG/L & $07 / 07 / 2009$ & & HCJ-166-09 \\
\hline GWA05406VL & TRIP BLANK & NA & 4-Methyl-2-pentanone & 5 & & $u$ & & UG/L & $07 / 07 / 2009$ & & HCJ-166-09 \\
\hline GWA05406VL & TRIP BLANK & NA & cis-1,3-Dichloropropylene & 1 & & $\mathrm{u}$ & & UG/L & $07 / 07 / 2009$ & & HCJ-166-09 \\
\hline GWA05406VL & TRIP BLANK & NA & Toluene & 1 & & U & & UG/L & $07 / 07 / 2009$ & & HCJ-166-09 \\
\hline GWA05406VL & TRIP BLANK & NA & trans-1,3-Dichloropropylene & 1 & & U & & UG/L & $07 / 07 / 2009$ & & HCJ-166-09 \\
\hline GWA05406VL & TRIP BLANK & NA & 1,1,2-Trichloroethane & 1 & & $\mathrm{u}$ & & UG/L & 07/07/2009 & & HCJ-166-09 \\
\hline GWA05406VL & TRIP BLANK & NA & 2-Hexanone & 5 & & U & & UG/L & $07 / 07 / 2009$ & & HCJ-166-09 \\
\hline GWA05406VL & TRIP BLANK & NA & Tetrachloroethylene & 0.671 & & BJ & $\mathrm{J}$ & UG/L & $07 / 07 / 2009$ & & HCJ-166-09 \\
\hline GWA05406VL & TRIP BLANK & NA & Dibromochloromethane & 1 & & U & & UG/L & $07 / 07 / 2009$ & & HCJ-166-09 \\
\hline GWA05406VL & TRIP BLANK & NA & 1,2-Dibromoethane & 1 & & $\mathrm{u}$ & & UG/L & $07 / 07 / 2009$ & & HCJ-166-09 \\
\hline GWA05406VL & TRIP BLANK & NA & Chlorobenzene & 1 & & U & & UG/L & $07 / 07 / 2009$ & & HCJ-166-09 \\
\hline GWA05406VL & TRIP BLANK & NA & Ethylbenzene & 1 & & $u$ & & UG/L & 07/07/2009 & & HCJ-166-09 \\
\hline GWA05406VL & TRIP BLANK & NA & Styrene & 1 & & $\mathrm{U}$ & & UG/L & 07/07/2009 & & HCJ-166-09 \\
\hline GWA05406VL & TRIP BLANK & NA & Bromoform & 1 & & U & & UG/L & $07 / 07 / 2009$ & & HCJ-166-09 \\
\hline GWA05406VL & TRIP BLANK & NA & 1,1,2,2-Tetrachloroethane & 1 & & $u$ & & UG/L & $07 / 07 / 2009$ & & HCJ-166-09 \\
\hline GWA05406VL & TRIP BLANK & NA & Isopropylbenzene & 1 & & $u$ & & UG/L & 07/07/2009 & & HCJ-166-09 \\
\hline GWA05406VL & TRIP BLANK & NA & 1,3-Dichlorobenzene & 1 & & $\mathrm{u}$ & & UG/L & $07 / 07 / 2009$ & & HCJ-166-09 \\
\hline GWA05406VL & TRIP BLANK & NA & 1,4-Dichlorobenzene & 1 & & U & & UG/L & $07 / 07 / 2009$ & & HCJ-166-09 \\
\hline GWA05406VL & TRIP BLANK & NA & 1,2-Dibromo-3-chloropropane & 1 & & $\mathrm{U}$ & & UG/L & $07 / 07 / 2009$ & & HCJ-166-09 \\
\hline GWA05406VL & TRIP BLANK & NA & Trichlorotrifluoroethane & 5 & & $\mathrm{u}$ & & UG/L & 07/07/2009 & & HCJ-166-09 \\
\hline GWA05406VL & TRIP BLANK & NA & Xylene (Total) & 1 & & $\mathrm{U}$ & & UG/L & 07/07/2009 & & HCJ-166-09 \\
\hline GWA05406VL & TRIP BLANK & NA & 1,2,4-Trichlorobenzene & 1 & & U & & UG/L & $07 / 07 / 2009$ & & HCJ-166-09 \\
\hline GWA05406VL & TRIP BLANK & NA & 1,2-Dichlorobenzene & 1 & & $u$ & & UG/L & $07 / 07 / 2009$ & & HCJ-166-09 \\
\hline GWA05303VL & FIELD BLANK & NA & Dichlorodifluoromethane & 1 & & $\mathrm{U}$ & & UG/L & 07/13/2009 & & HCJ-167-09 \\
\hline GWA05303VL & FIELD BLANK & NA & Chloromethane & 1 & & $\mathrm{U}$ & & UG/L & 07/13/2009 & & HCJ-167-09 \\
\hline GWA05303VL & FIELD BLANK & NA & Vinyl Chloride & 1 & & $U$ & & UG/L & $07 / 13 / 2009$ & & HCJ-167-09 \\
\hline GWA05303VL & FIELD BLANK & NA & Bromomethane & 1 & & $\mathrm{U}$ & & UG/L & $07 / 13 / 2009$ & & HCJ-167-09 \\
\hline GWA05303VL & FIELD BLANK & NA & Chloroethane & 1 & & $\mathrm{U}$ & & UG/L & $07 / 13 / 2009$ & & HCJ-167-09 \\
\hline GWA05303VL & FIELD BLANK & NA & Trichlorofluoromethane & 1 & & $\mathrm{U}$ & & UG/L & 07/13/2009 & & HCJ-167-09 \\
\hline
\end{tabular}


Table A-2. (continued).

\begin{tabular}{|c|c|c|c|c|c|c|c|c|c|c|c|}
\hline $\begin{array}{c}\text { Field Sample } \\
\text { Number }\end{array}$ & Location & Depth & Compound & $\begin{array}{c}\text { Sample } \\
\text { Result }\end{array}$ & $\begin{array}{c}\text { Sample } \\
\text { Error }\end{array}$ & $\begin{array}{c}\text { Result } \\
\text { Qualifier }\end{array}$ & $\begin{array}{c}\text { Validation } \\
\text { Flag }\end{array}$ & $\begin{array}{c}\text { Sample } \\
\text { Units }\end{array}$ & $\begin{array}{c}\text { Date Sample } \\
\text { Collected }\end{array}$ & MDA & L\&V Report Number \\
\hline GWA05303VL & FIELD BLANK & NA & Acetone & 5 & & $\mathrm{U}$ & & UG/L & $07 / 13 / 2009$ & & HCJ-167-09 \\
\hline GWA05303VL & FIELD BLANK & NA & 1,1-Dichloroethene & 1 & & U & & UG/L & $07 / 13 / 2009$ & & HCJ-167-09 \\
\hline GWA05303VL & FIELD BLANK & NA & Methyl acetate & 5 & & U & & UG/L & $07 / 13 / 2009$ & & HCJ-167-09 \\
\hline GWA05303VL & FIELD BLANK & NA & Methylene Chloride & 5 & & $U$ & & UG/L & $07 / 13 / 2009$ & & HCJ-167-09 \\
\hline GWA05303VL & FIELD BLANK & NA & Carbon disulfide & 5 & & $U$ & & UG/L & $07 / 13 / 2009$ & & HCJ-167-09 \\
\hline GWA05303VL & FIELD BLANK & NA & Tert-butyl methyl ether & 1 & & U & & UG/L & $07 / 13 / 2009$ & & HCJ-167-09 \\
\hline GWA05303VL & FIELD BLANK & NA & trans-1,2-Dichloroethylene & 1 & & U & & UG/L & 07/13/2009 & & HCJ-167-09 \\
\hline GWA05303VL & FIELD BLANK & NA & 1,1-Dichloroethane & 1 & & U & & UG/L & 07/13/2009 & & HCJ-167-09 \\
\hline GWA05303VL & FIELD BLANK & NA & 2-Butanone & 5 & & U & & UG/L & $07 / 13 / 2009$ & & HCJ-167-09 \\
\hline GWA05303VL & FIELD BLANK & NA & cis-1,2-Dichloroethylene & 1 & & U & & UG/L & $07 / 13 / 2009$ & & HCJ-167-09 \\
\hline GWA05303VL & FIELD BLANK & NA & Chloroform & 2.59 & & & J & UG/L & $07 / 13 / 2009$ & & HCJ-167-09 \\
\hline GWA05303VL & FIELD BLANK & NA & 1,1,1-Trichloroethane & 1 & & $U$ & & UG/L & 07/13/2009 & & HCJ-167-09 \\
\hline GWA05303VL & FIELD BLANK & NA & Cyclohexane & 1 & & U & & UG/L & $07 / 13 / 2009$ & & HCJ-167-09 \\
\hline GWA05303VL & FIELD BLANK & NA & Carbon tetrachloride & 1 & & $U$ & & UG/L & $07 / 13 / 2009$ & & HCJ-167-09 \\
\hline GWA05303VL & FIELD BLANK & NA & 1,2-Dichloroethane & 1 & & $U$ & & UG/L & 07/13/2009 & & HCJ-167-09 \\
\hline GWA05303VL & FIELD BLANK & NA & Benzene & 1 & & U & & UG/L & $07 / 13 / 2009$ & & HCJ-167-09 \\
\hline GWA05303VL & FIELD BLANK & NA & Trichloroethylene & 1 & & $U$ & & UG/L & $07 / 13 / 2009$ & & HCJ-167-09 \\
\hline GWA05303VL & FIELD BLANK & NA & 1,2-Dichloropropane & 1 & & $U$ & & UG/L & $07 / 13 / 2009$ & & HCJ-167-09 \\
\hline GWA05303VL & FIELD BLANK & NA & Methyl cyclohexane & 1 & & $U$ & & UG/L & $07 / 13 / 2009$ & & HCJ-167-09 \\
\hline GWA05303VL & FIELD BLANK & NA & Bromodichloromethane & 1 & & $U$ & & UG/L & $07 / 13 / 2009$ & & HCJ-167-09 \\
\hline GWA05303VL & FIELD BLANK & NA & 4-Methyl-2-pentanone & 5 & & U & & UG/L & $07 / 13 / 2009$ & & HCJ-167-09 \\
\hline GWA05303VL & FIELD BLANK & NA & cis-1,3-Dichloropropylene & 1 & & $\mathrm{U}$ & & UG/L & $07 / 13 / 2009$ & & HCJ-167-09 \\
\hline GWA05303VL & FIELD BLANK & NA & Toluene & 1 & & $\mathrm{U}$ & & UG/L & $07 / 13 / 2009$ & & HCJ-167-09 \\
\hline GWA05303VL & FIELD BLANK & NA & trans-1,3-Dichloropropylene & 1 & & $U$ & & UG/L & 07/13/2009 & & HCJ-167-09 \\
\hline GWA05303VL & FIELD BLANK & NA & 1,1,2-Trichloroethane & 1 & & U & & UG/L & 07/13/2009 & & HCJ-167-09 \\
\hline GWA05303VL & FIELD BLANK & NA & 2-Hexanone & 5 & & $U$ & & UG/L & $07 / 13 / 2009$ & & HCJ-167-09 \\
\hline GWA05303VL & FIELD BLANK & NA & Tetrachloroethylene & 1 & & U & & UG/L & 07/13/2009 & & HCJ-167-09 \\
\hline GWA05303VL & FIELD BLANK & NA & Dibromochloromethane & 1 & & U & & UG/L & $07 / 13 / 2009$ & & HCJ-167-09 \\
\hline GWA05303VL & FIELD BLANK & NA & 1,2-Dibromoethane & 1 & & $\mathrm{U}$ & & UG/L & $07 / 13 / 2009$ & & HCJ-167-09 \\
\hline GWA05303VL & FIELD BLANK & NA & Chlorobenzene & 1 & & $\mathrm{U}$ & & UG/L & $07 / 13 / 2009$ & & HCJ-167-09 \\
\hline GWA05303VL & FIELD BLANK & NA & Ethylbenzene & 1 & & U & & UG/L & $07 / 13 / 2009$ & & HCJ-167-09 \\
\hline GWA05303VL & FIELD BLANK & NA & Styrene & 1 & & 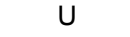 & & UG/L & $07 / 13 / 2009$ & & HCJ-167-09 \\
\hline GWA05303VL & FIELD BLANK & NA & Bromoform & 1 & & $U$ & & UG/L & $07 / 13 / 2009$ & & HCJ-167-09 \\
\hline GWA05303VL & FIELD BLANK & NA & 1,1,2,2-Tetrachloroethane & 1 & & $\mathrm{U}$ & & UG/L & $07 / 13 / 2009$ & & HCJ-167-09 \\
\hline GWA05303VL & FIELD BLANK & NA & Isopropylbenzene & 1 & & $u$ & & UG/L & $07 / 13 / 2009$ & & HCJ-167-09 \\
\hline GWA05303VL & FIELD BLANK & NA & 1,3-Dichlorobenzene & 1 & & $\mathrm{U}$ & & UG/L & $07 / 13 / 2009$ & & HCJ-167-09 \\
\hline GWA05303VL & FIELD BLANK & NA & 1,4-Dichlorobenzene & 1 & & $\mathrm{U}$ & & UG/L & $07 / 13 / 2009$ & & HCJ-167-09 \\
\hline GWA05303VL & FIELD BLANK & NA & 1,2-Dibromo-3-chloropropane & 1 & & U & & UG/L & $07 / 13 / 2009$ & & HCJ-167-09 \\
\hline GWA05303VL & FIELD BLANK & NA & Trichlorotrifluoroethane & 5 & & $u$ & & UG/L & $07 / 13 / 2009$ & & HCJ-167-09 \\
\hline GWA05303VL & FIELD BLANK & NA & Xylene (Total) & 1 & & $U$ & & UG/L & $07 / 13 / 2009$ & & HCJ-167-09 \\
\hline GWA05303VL & FIELD BLANK & NA & 1,2,4-Trichlorobenzene & 1 & & U & & UG/L & $07 / 13 / 2009$ & & HCJ-167-09 \\
\hline GWA05303VL & FIELD BLANK & NA & 1,2-Dichlorobenzene & 1 & & $u$ & & UG/L & $07 / 13 / 2009$ & & HCJ-167-09 \\
\hline GWA05303VL & FIELD BLANK & NA & unknown & 13.8 & & $\mathrm{~J}$ & & UG/L & $07 / 13 / 2009$ & & \\
\hline GWA05303VL & FIELD BLANK & NA & unknown siloxane & 21.8 & & $\mathrm{~J}$ & & UG/L & $07 / 13 / 2009$ & & \\
\hline
\end{tabular}


Table A-2. (continued).

\begin{tabular}{|c|c|c|c|c|c|c|c|c|c|c|c|}
\hline $\begin{array}{c}\text { Field Sample } \\
\text { Number }\end{array}$ & Location & Depth & Compound & $\begin{array}{c}\text { Sample } \\
\text { Result }\end{array}$ & $\begin{array}{c}\text { Sample } \\
\text { Error }\end{array}$ & $\begin{array}{c}\text { Result } \\
\text { Qualifier }\end{array}$ & $\begin{array}{c}\text { Validation } \\
\text { Flag }\end{array}$ & $\begin{array}{c}\text { Sample } \\
\text { Units }\end{array}$ & $\begin{array}{c}\text { Date Sample } \\
\text { Collected }\end{array}$ & MDA & L\&V Report Number \\
\hline GWA05407VL & TRIP BLANK & NA & Dichlorodifluoromethane & 1 & & $\mathrm{U}$ & & UG/L & $07 / 13 / 2009$ & & HCJ-167-09 \\
\hline GWA05407VL & TRIP BLANK & NA & Chloromethane & 1 & & $\mathrm{U}$ & & UG/L & 07/13/2009 & & HCJ-167-09 \\
\hline GWA05407VL & TRIP BLANK & NA & Vinyl Chloride & 1 & & $\mathrm{U}$ & & UG/L & 07/13/2009 & & HCJ-167-09 \\
\hline GWA05407VL & TRIP BLANK & NA & Bromomethane & 1 & & $\mathrm{U}$ & & UG/L & 07/13/2009 & & HCJ-167-09 \\
\hline GWA05407VL & TRIP BLANK & NA & Chloroethane & 1 & & $\mathrm{U}$ & & UG/L & 07/13/2009 & & HCJ-167-09 \\
\hline GWA05407VL & TRIP BLANK & NA & Trichlorofluoromethane & 1 & & U & & UG/L & 07/13/2009 & & HCJ-167-09 \\
\hline GWA05407VL & TRIP BLANK & NA & Acetone & 1.78 & & $\mathrm{~J}$ & $\mathrm{~J}$ & UG/L & 07/13/2009 & & HCJ-167-09 \\
\hline GWA05407VL & TRIP BLANK & NA & 1,1-Dichloroethene & 1 & & U & & UG/L & 07/13/2009 & & HCJ-167-09 \\
\hline GWA05407VL & TRIP BLANK & NA & Methyl acetate & 5 & & $\mathrm{U}$ & & UG/L & 07/13/2009 & & HCJ-167-09 \\
\hline GWA05407VL & TRIP BLANK & NA & Methylene Chloride & 5 & & $\mathrm{U}$ & & UG/L & 07/13/2009 & & HCJ-167-09 \\
\hline GWA05407VL & TRIP BLANK & NA & Carbon disulfide & 5 & & $\mathrm{U}$ & & UG/L & 07/13/2009 & & HCJ-167-09 \\
\hline GWA05407VL & TRIP BLANK & NA & Tert-butyl methyl ether & 1 & & $\mathrm{U}$ & & UG/L & 07/13/2009 & & HCJ-167-09 \\
\hline GWA05407VL & TRIP BLANK & NA & trans-1,2-Dichloroethylene & 1 & & $\mathrm{U}$ & & UG/L & 07/13/2009 & & HCJ-167-09 \\
\hline GWA05407VL & TRIP BLANK & NA & 1,1-Dichloroethane & 1 & & $\mathrm{U}$ & & UG/L & 07/13/2009 & & HCJ-167-09 \\
\hline GWA05407VL & TRIP BLANK & NA & 2-Butanone & 5 & & $\mathrm{U}$ & & UG/L & 07/13/2009 & & HCJ-167-09 \\
\hline GWA05407VL & TRIP BLANK & NA & cis-1,2-Dichloroethylene & 1 & & $\mathrm{U}$ & & UG/L & 07/13/2009 & & HCJ-167-09 \\
\hline GWA05407VL & TRIP BLANK & NA & Chloroform & 2.72 & & & $\mathrm{~J}$ & UG/L & 07/13/2009 & & HCJ-167-09 \\
\hline GWA05407VL & TRIP BLANK & NA & 1,1,1-Trichloroethane & 1 & & $\mathrm{U}$ & & UG/L & 07/13/2009 & & HCJ-167-09 \\
\hline GWA05407VL & TRIP BLANK & NA & Cyclohexane & 1 & & $\mathrm{U}$ & & UG/L & 07/13/2009 & & HCJ-167-09 \\
\hline GWA05407VL & TRIP BLANK & NA & Carbon tetrachloride & 1 & & U & & UG/L & 07/13/2009 & & HCJ-167-09 \\
\hline GWA05407VL & TRIP BLANK & NA & 1,2-Dichloroethane & 1 & & $\mathrm{U}$ & & UG/L & 07/13/2009 & & HCJ-167-09 \\
\hline GWA05407VL & TRIP BLANK & NA & Benzene & 1 & & $\mathrm{U}$ & & UG/L & 07/13/2009 & & HCJ-167-09 \\
\hline GWA05407VL & TRIP BLANK & NA & Trichloroethylene & 1 & & $\mathrm{U}$ & & UG/L & $07 / 13 / 2009$ & & HCJ-167-09 \\
\hline GWA05407VL & TRIP BLANK & NA & 1,2-Dichloropropane & 1 & & $\mathrm{U}$ & & UG/L & 07/13/2009 & & HCJ-167-09 \\
\hline GWA05407VL & TRIP BLANK & NA & Methyl cyclohexane & 1 & & $\mathrm{U}$ & & UG/L & 07/13/2009 & & HCJ-167-09 \\
\hline GWA05407VL & TRIP BLANK & NA & Bromodichloromethane & 1 & & $\mathrm{U}$ & & UG/L & 07/13/2009 & & HCJ-167-09 \\
\hline GWA05407VL & TRIP BLANK & NA & 4-Methyl-2-pentanone & 5 & & U & & UG/L & 07/13/2009 & & HCJ-167-09 \\
\hline GWA05407VL & TRIP BLANK & NA & cis-1,3-Dichloropropylene & 1 & & $\mathrm{U}$ & & UG/L & 07/13/2009 & & HCJ-167-09 \\
\hline GWA05407VL & TRIP BLANK & NA & Toluene & 1 & & $\mathrm{U}$ & & UG/L & 07/13/2009 & & HCJ-167-09 \\
\hline GWA05407VL & TRIP BLANK & NA & trans-1,3-Dichloropropylene & 1 & & $\mathrm{U}$ & & UG/L & $07 / 13 / 2009$ & & HCJ-167-09 \\
\hline GWA05407VL & TRIP BLANK & NA & 1,1,2-Trichloroethane & 1 & & $U$ & & UG/L & 07/13/2009 & & HCJ-167-09 \\
\hline GWA05407VL & TRIP BLANK & NA & 2-Hexanone & 5 & & $\mathrm{U}$ & & UG/L & 07/13/2009 & & HCJ-167-09 \\
\hline GWA05407VL & TRIP BLANK & NA & Tetrachloroethylene & 1 & & $\mathrm{U}$ & & UG/L & 07/13/2009 & & HCJ-167-09 \\
\hline GWA05407VL & TRIP BLANK & NA & Dibromochloromethane & 1 & & $\mathrm{U}$ & & UG/L & 07/13/2009 & & HCJ-167-09 \\
\hline GWA05407VL & TRIP BLANK & NA & 1,2-Dibromoethane & 1 & & $\mathrm{U}$ & & UG/L & 07/13/2009 & & HCJ-167-09 \\
\hline GWA05407VL & TRIP BLANK & NA & Chlorobenzene & 1 & & $U$ & & UG/L & 07/13/2009 & & HCJ-167-09 \\
\hline GWA05407VL & TRIP BLANK & NA & Ethylbenzene & 1 & & $\mathrm{U}$ & & UG/L & 07/13/2009 & & HCJ-167-09 \\
\hline GWA05407VL & TRIP BLANK & NA & Styrene & 1 & & $\mathrm{U}$ & & UG/L & 07/13/2009 & & HCJ-167-09 \\
\hline GWA05407VL & TRIP BLANK & NA & Bromoform & 1 & & $\mathrm{U}$ & & UG/L & 07/13/2009 & & HCJ-167-09 \\
\hline GWA05407VL & TRIP BLANK & NA & 1,1,2,2-Tetrachloroethane & 1 & & $\mathrm{U}$ & & UG/L & 07/13/2009 & & HCJ-167-09 \\
\hline GWA05407VL & TRIP BLANK & NA & Isopropylbenzene & 1 & & $\mathrm{U}$ & & UG/L & 07/13/2009 & & HCJ-167-09 \\
\hline GWA05407VL & TRIP BLANK & NA & 1,3-Dichlorobenzene & 1 & & $\mathrm{U}$ & & UG/L & 07/13/2009 & & HCJ-167-09 \\
\hline GWA05407VL & TRIP BLANK & NA & 1,4-Dichlorobenzene & 1 & & $\mathrm{U}$ & & UG/L & 07/13/2009 & & HCJ-167-09 \\
\hline GWA05407VL & TRIP BLANK & NA & 1,2-Dibromo-3-chloropropane & 1 & & $\mathrm{U}$ & & UG/L & 07/13/2009 & & HCJ-167-09 \\
\hline
\end{tabular}


Table A-2. (continued).

\begin{tabular}{|c|c|c|c|c|c|c|c|c|c|c|c|}
\hline $\begin{array}{c}\text { Field Sample } \\
\text { Number }\end{array}$ & Location & Depth & Compound & $\begin{array}{c}\text { Sample } \\
\text { Result }\end{array}$ & $\begin{array}{l}\text { Sample } \\
\text { Error }\end{array}$ & $\begin{array}{c}\text { Result } \\
\text { Qualifier }\end{array}$ & $\begin{array}{c}\text { Validation } \\
\text { Flag }\end{array}$ & $\begin{array}{c}\text { Sample } \\
\text { Units }\end{array}$ & $\begin{array}{c}\text { Date Sample } \\
\text { Collected }\end{array}$ & MDA & L\&V Report Number \\
\hline GWA05407VL & TRIP BLANK & NA & Trichlorotrifluoroethane & 5 & & $U$ & & UG/L & $07 / 13 / 2009$ & & HCJ-167-09 \\
\hline GWA05407VL & TRIP BLANK & NA & Xylene (Total) & 1 & & U & & UG/L & 07/13/2009 & & HCJ-167-09 \\
\hline GWA05407VL & TRIP BLANK & NA & 1,2,4-Trichlorobenzene & 1 & & $\mathrm{U}$ & & UG/L & 07/13/2009 & & HCJ-167-09 \\
\hline GWA05407VL & TRIP BLANK & NA & 1,2-Dichlorobenzene & 1 & & $\mathrm{U}$ & & UG/L & 07/13/2009 & & HCJ-167-09 \\
\hline GWA05407VL & TRIP BLANK & NA & unknown & 14.4 & & $\mathrm{~J}$ & & UG/L & 07/13/2009 & & \\
\hline GWA05407VL & TRIP BLANK & NA & unknown siloxane & 25.3 & & $\mathrm{~J}$ & & UG/L & 07/13/2009 & & \\
\hline GWA05302R8 & FIELD BLANK & NA & Tritium & $2.33 \mathrm{E}+01$ & $8.30 E+01$ & & $\mathrm{U}$ & $\mathrm{PCI} / \mathrm{L}$ & 07/02/2009 & $2.87 \mathrm{E}+02$ & BAM-116-09 \\
\hline GWA05303R8 & FIELD BLANK & NA & Tritium & $-3.87 \mathrm{E}+01$ & $8.07 \mathrm{E}+01$ & & U & $\mathrm{PCI} / \mathrm{L}$ & 07/13/2009 & $2.86 \mathrm{E}+02$ & BAM-116-09 \\
\hline GWA05302RH & FIELD BLANK & NA & Gross Alpha & 2.77E-01 & $2.47 \mathrm{E}-01$ & & U & $\mathrm{PCI} / \mathrm{L}$ & 07/02/2009 & 8.92E-01 & BAM-115-09 \\
\hline GWA05302RH & FIELD BLANK & NA & Gross Beta & $2.65 \mathrm{E}-01$ & 3.32E-01 & & $\mathrm{U}$ & $\mathrm{PCl} / \mathrm{L}$ & 07/02/2009 & $1.16 \mathrm{E}+00$ & BAM-115-09 \\
\hline GWA05303RH & FIELD BLANK & NA & Gross Alpha & $-4.90 \mathrm{E}-02$ & $2.58 \mathrm{E}-01$ & & $\mathrm{U}$ & $\mathrm{PCl} / \mathrm{L}$ & 07/13/2009 & $1.13 E+00$ & BAM-115-09 \\
\hline GWA05303RH & FIELD BLANK & NA & Gross Beta & $1.04 \mathrm{E}+00$ & $6.01 \mathrm{E}-01$ & & $U$ & $\mathrm{PCI} / \mathrm{L}$ & 07/13/2009 & $1.95 \mathrm{E}+00$ & BAM-115-09 \\
\hline GWA05302RH & FIELD BLANK & NA & Strontium-90 & $1.28 \mathrm{E}-01$ & 8.10E-02 & & U & $\mathrm{PCl} / \mathrm{L}$ & $07 / 02 / 2009$ & 2.67E-01 & BAM-115-09 \\
\hline GWA05303RH & FIELD BLANK & NA & Strontium-90 & $2.06 \mathrm{E}-02$ & $1.28 \mathrm{E}-01$ & & U & $\mathrm{PCl} / \mathrm{L}$ & 07/13/2009 & $4.55 \mathrm{E}-01$ & BAM-115-09 \\
\hline GWA05302RH & FIELD BLANK & NA & Technetium-99 & $-2.25 E+00$ & $2.33 E+00$ & & $U$ & $\mathrm{PCl} / \mathrm{L}$ & 07/02/2009 & $8.05 \mathrm{E}+00$ & BAM-115-09 \\
\hline GWA05303RH & FIELD BLANK & NA & Technetium-99 & $0.00 \mathrm{E}+00$ & $2.37 \mathrm{E}+00$ & & $U$ & $\mathrm{PCl} / \mathrm{L}$ & 07/13/2009 & $8.07 \mathrm{E}+00$ & BAM-115-09 \\
\hline GWA05302UX & FIELD BLANK & NA & lodine-129 & $-8.17 \mathrm{E}-03$ & $2.72 \mathrm{E}-02$ & & $\mathrm{U}$ & $\mathrm{PCl} / \mathrm{L}$ & $07 / 02 / 2009$ & $8.80 \mathrm{E}-02$ & BAM-117-09 \\
\hline GWA05303UX & FIELD BLANK & NA & lodine-129 & $-4.36 \mathrm{E}-02$ & $1.45 \mathrm{E}-02$ & & U & $\mathrm{PCl} / \mathrm{L}$ & $07 / 13 / 2009$ & $3.26 \mathrm{E}-02$ & BAM-117-09 \\
\hline GWA05301R8 & FIELD BLANK & NA & Tritium & $-4.95 E+01$ & $1.05 E+02$ & & $U$ & $\mathrm{PCl} / \mathrm{L}$ & 06/17/2009 & $3.86 \mathrm{E}+02$ & BAM-119-09 \\
\hline GWA05301RH & FIELD BLANK & NA & Gross Alpha & $1.26 \mathrm{E}+00$ & 4.90E-01 & & UJ & $\mathrm{PCl} / \mathrm{L}$ & $06 / 17 / 2009$ & $1.40 \mathrm{E}+00$ & BAM-120-09 \\
\hline GWA05301RH & FIELD BLANK & NA & Gross Beta & $2.77 \mathrm{E}+00$ & $6.07 \mathrm{E}-01$ & & & $\mathrm{PCl} / \mathrm{L}$ & 06/17/2009 & $1.59 \mathrm{E}+00$ & BAM-120-09 \\
\hline GWA05301RH & FIELD BLANK & NA & Strontium-90 & $9.75 \mathrm{E}-02$ & $1.05 \mathrm{E}-01$ & & U & $\mathrm{PCl} / \mathrm{L}$ & 06/17/2009 & 3.57E-01 & BAM-120-09 \\
\hline GWA05301RH & FIELD BLANK & NA & Technetium-99 & $-1.76 \mathrm{E}+00$ & $1.63 E+00$ & & $U$ & $\mathrm{PCl} / \mathrm{L}$ & 06/17/2009 & $5.85 \mathrm{E}+00$ & BAM-120-09 \\
\hline GWA05301UX & FIELD BLANK & NA & lodine-129 & $-3.18 \mathrm{E}-02$ & 3.08E-02 & & $\mathrm{U}$ & $\mathrm{PCl} / \mathrm{L}$ & $06 / 17 / 2009$ & $9.23 \mathrm{E}-02$ & BAM-118-09 \\
\hline GWA030012X & USGS-001 & 588 & Aluminum & 15 & & U & & UG/L & 06/03/2009 & & HCJ-161-09 \\
\hline GWA030012X & USGS-001 & 588 & Antimony & 1 & & U & & UG/L & 06/03/2009 & & HCJ-161-09 \\
\hline GWA030012X & USGS-001 & 588 & Arsenic & 1.6 & & $u$ & & UG/L & 06/03/2009 & & HCJ-161-09 \\
\hline GWA030012X & USGS-001 & 588 & Barium & 24.1 & & B & & UG/L & $06 / 03 / 2009$ & & HCJ-161-09 \\
\hline GWA030012X & USGS-001 & 588 & Beryllium & 0.1 & & U & & UG/L & $06 / 03 / 2009$ & & HCJ-161-09 \\
\hline GWA030012X & USGS-001 & 588 & Cadmium & 0.11 & & U & & UG/L & 06/03/2009 & & HCJ-161-09 \\
\hline GWA030012X & USGS-001 & 588 & Calcium & 27800 & & & & UG/L & $06 / 03 / 2009$ & & HCJ-161-09 \\
\hline GWA030012X & USGS-001 & 588 & Chromium & 2.34 & & B & & UG/L & 06/03/2009 & & HCJ-161-09 \\
\hline GWA030012X & USGS-001 & 588 & Cobalt & 3.78 & & B & & UG/L & 06/03/2009 & & HCJ-161-09 \\
\hline GWA030012X & USGS-001 & 588 & Copper & 0.811 & & B & & UG/L & 06/03/2009 & & HCJ-161-09 \\
\hline GWA030012X & USGS-001 & 588 & Iron & 176 & & & & UG/L & 06/03/2009 & & HCJ-161-09 \\
\hline GWA030012X & USGS-001 & 588 & Lead & 0.5 & & U & & UG/L & 06/03/2009 & & HCJ-161-09 \\
\hline GWA030012X & USGS-001 & 588 & Magnesium & 11000 & & & & UG/L & 06/03/2009 & & HCJ-161-09 \\
\hline GWA030012X & USGS-001 & 588 & Manganese & 11.4 & & B & & UG/L & 06/03/2009 & & HCJ-161-09 \\
\hline GWA030012X & USGS-001 & 588 & Mercury & 0.067 & & U & & UG/L & 06/03/2009 & & HCJ-161-09 \\
\hline GWA030012X & USGS-001 & 588 & Nickel & 1.82 & & B & & UG/L & 06/03/2009 & & HCJ-161-09 \\
\hline GWA030012X & USGS-001 & 588 & Potassium & 2730 & & B & & UG/L & 06/03/2009 & & HCJ-161-09 \\
\hline GWA030012X & USGS-001 & 588 & Selenium & 1 & & U & & UG/L & 06/03/2009 & & HCJ-161-09 \\
\hline GWA030012X & USGS-001 & 588 & Silver & 0.2 & & U & & UG/L & 06/03/2009 & & HCJ-161-09 \\
\hline GWA030012X & USGS-001 & 588 & Sodium & 13300 & & & & UG/L & $06 / 03 / 2009$ & & HCJ-161-09 \\
\hline
\end{tabular}


Table A-2. (continued).

\begin{tabular}{|c|c|c|c|c|c|c|c|c|c|c|c|}
\hline $\begin{array}{c}\text { Field Sample } \\
\text { Number }\end{array}$ & Location & Depth & Compound & $\begin{array}{l}\text { Sample } \\
\text { Result }\end{array}$ & $\begin{array}{c}\text { Sample } \\
\text { Error }\end{array}$ & $\begin{array}{c}\text { Result } \\
\text { Qualifier }\end{array}$ & $\begin{array}{c}\text { Validation } \\
\text { Flag }\end{array}$ & $\begin{array}{c}\text { Sample } \\
\text { Units }\end{array}$ & $\begin{array}{c}\text { Date Sample } \\
\text { Collected }\end{array}$ & MDA & L\&V Report Number \\
\hline GWA030012X & USGS-001 & 588 & Strontium & 117 & & & & UG/L & $06 / 03 / 2009$ & & HCJ-161-09 \\
\hline GWA030012X & USGS-001 & 588 & Thallium & 0.3 & & U & & UG/L & $06 / 03 / 2009$ & & HCJ-161-09 \\
\hline GWA030012X & USGS-001 & 588 & Uranium & 2.05 & & B & & UG/L & 06/03/2009 & & HCJ-161-09 \\
\hline GWA030012X & USGS-001 & 588 & Vanadium & 3 & & $\mathrm{U}$ & & UG/L & $06 / 03 / 2009$ & & HCJ-161-09 \\
\hline GWA030012X & USGS-001 & 588 & Zinc & 3.26 & & B & & UG/L & $06 / 03 / 2009$ & & HCJ-161-09 \\
\hline GWA03001A1 & USGS-001 & 588 & Alkalinity, Total as $\mathrm{CaCO} 3$ & 125 & & & & $M G / L$ & 06/03/2009 & & HCJ-157-09 \\
\hline GWA03001AN & USGS-001 & 588 & Bromide & 0 & & $\mathrm{U}$ & & $M G / L$ & $06 / 03 / 2009$ & & HCJ-159-09 \\
\hline GWA03001AN & USGS-001 & 588 & Chloride & 12.8 & & & & $M G / L$ & $06 / 03 / 2009$ & & HCJ-159-09 \\
\hline GWA03001AN & USGS-001 & 588 & Fluoride & 0.707 & & & & $M G / L$ & $06 / 03 / 2009$ & & HCJ-159-09 \\
\hline GWA03001AN & USGS-001 & 588 & Sulfate & 13 & & & & $M G / L$ & 06/03/2009 & & HCJ-159-09 \\
\hline GWA03001N2 & USGS-001 & 588 & Nitrogen, Nitrate/Nitrite & 1.16 & & & & $M G / L$ & $06 / 03 / 2009$ & & HCJ-155-09 \\
\hline GWA03001VL & USGS-001 & 588 & Dichlorodifluoromethane & 1 & & $U$ & & UG/L & $06 / 03 / 2009$ & & HCJ-148-09 \\
\hline GWA03001VL & USGS-001 & 588 & Chloromethane & 1 & & $u$ & & UG/L & $06 / 03 / 2009$ & & HCJ-148-09 \\
\hline GWA03001VL & USGS-001 & 588 & Vinyl Chloride & 1 & & $\mathrm{U}$ & & UG/L & $06 / 03 / 2009$ & & HCJ-148-09 \\
\hline GWA03001VL & USGS-001 & 588 & Bromomethane & 1 & & U & & UG/L & $06 / 03 / 2009$ & & HCJ-148-09 \\
\hline GWA03001VL & USGS-001 & 588 & Chloroethane & 1 & & U & & UG/L & $06 / 03 / 2009$ & & HCJ-148-09 \\
\hline GWA03001VL & USGS-001 & 588 & Trichlorofluoromethane & 1 & & $\mathrm{U}$ & & UG/L & 06/03/2009 & & HCJ-148-09 \\
\hline GWA03001VL & USGS-001 & 588 & Acetone & 5 & & $\mathrm{U}$ & & UG/L & $06 / 03 / 2009$ & & HCJ-148-09 \\
\hline GWA03001VL & USGS-001 & 588 & 1,1-Dichloroethene & 1 & & U & & UG/L & $06 / 03 / 2009$ & & HCJ-148-09 \\
\hline GWA03001VL & USGS-001 & 588 & Methyl acetate & 5 & & $u$ & & UG/L & $06 / 03 / 2009$ & & HCJ-148-09 \\
\hline GWA03001VL & USGS-001 & 588 & Methylene Chloride & 5 & & $\mathrm{U}$ & & UG/L & 06/03/2009 & & HCJ-148-09 \\
\hline GWA03001VL & USGS-001 & 588 & Carbon disulfide & 5 & & U & & UG/L & $06 / 03 / 2009$ & & HCJ-148-09 \\
\hline GWA03001VL & USGS-001 & 588 & Tert-butyl methyl ether & 1 & & U & & UG/L & $06 / 03 / 2009$ & & HCJ-148-09 \\
\hline GWA03001VL & USGS-001 & 588 & trans-1,2-Dichloroethylene & 1 & & $U$ & & UG/L & $06 / 03 / 2009$ & & HCJ-148-09 \\
\hline GWA03001VL & USGS-001 & 588 & 1,1-Dichloroethane & 1 & & $\mathrm{U}$ & & UG/L & $06 / 03 / 2009$ & & HCJ-148-09 \\
\hline GWA03001VL & USGS-001 & 588 & 2-Butanone & 5 & & U & & UG/L & $06 / 03 / 2009$ & & HCJ-148-09 \\
\hline GWA03001VL & USGS-001 & 588 & cis-1,2-Dichloroethylene & 1 & & $\mathrm{U}$ & & UG/L & 06/03/2009 & & HCJ-148-09 \\
\hline GWA03001VL & USGS-001 & 588 & Chloroform & 1 & & $\mathrm{U}$ & & UG/L & 06/03/2009 & & HCJ-148-09 \\
\hline GWA03001VL & USGS-001 & 588 & 1,1,1-Trichloroethane & 1 & & U & & UG/L & $06 / 03 / 2009$ & & HCJ-148-09 \\
\hline GWA03001VL & USGS-001 & 588 & Cyclohexane & 1 & & U & & UG/L & $06 / 03 / 2009$ & & HCJ-148-09 \\
\hline GWA03001VL & USGS-001 & 588 & Carbon tetrachloride & 1 & & $u$ & & UG/L & $06 / 03 / 2009$ & & HCJ-148-09 \\
\hline GWA03001VL & USGS-001 & 588 & 1,2-Dichloroethane & 1 & & $\mathrm{u}$ & & UG/L & $06 / 03 / 2009$ & & HCJ-148-09 \\
\hline GWA03001VL & USGS-001 & 588 & Benzene & 1 & & U & & UG/L & $06 / 03 / 2009$ & & HCJ-148-09 \\
\hline GWA03001VL & USGS-001 & 588 & Trichloroethylene & 1 & & $u$ & & UG/L & $06 / 03 / 2009$ & & HCJ-148-09 \\
\hline GWA03001VL & USGS-001 & 588 & 1,2-Dichloropropane & 1 & & $\mathrm{u}$ & & UG/L & 06/03/2009 & & HCJ-148-09 \\
\hline GWA03001VL & USGS-001 & 588 & Methyl cyclohexane & 1 & & U & & UG/L & $06 / 03 / 2009$ & & HCJ-148-09 \\
\hline GWA03001VL & USGS-001 & 588 & Bromodichloromethane & 1 & & U & & UG/L & $06 / 03 / 2009$ & & HCJ-148-09 \\
\hline GWA03001VL & USGS-001 & 588 & 4-Methyl-2-pentanone & 5 & & $u$ & & UG/L & $06 / 03 / 2009$ & & HCJ-148-09 \\
\hline GWA03001VL & USGS-001 & 588 & cis-1,3-Dichloropropylene & 1 & & $\mathrm{U}$ & & UG/L & $06 / 03 / 2009$ & & HCJ-148-09 \\
\hline GWA03001VL & USGS-001 & 588 & Toluene & 1 & & $\mathrm{U}$ & & UG/L & $06 / 03 / 2009$ & & HCJ-148-09 \\
\hline GWA03001VL & USGS-001 & 588 & trans-1,3-Dichloropropylene & 1 & & $\mathrm{U}$ & & UG/L & $06 / 03 / 2009$ & & HCJ-148-09 \\
\hline GWA03001VL & USGS-001 & 588 & 1,1,2-Trichloroethane & 1 & & $\mathrm{U}$ & & UG/L & $06 / 03 / 2009$ & & HCJ-148-09 \\
\hline GWA03001VL & USGS-001 & 588 & 2-Hexanone & 5 & & $u$ & & UG/L & $06 / 03 / 2009$ & & HCJ-148-09 \\
\hline GWA03001VL & USGS-001 & 588 & Tetrachloroethylene & 1 & & $\mathrm{U}$ & & UG/L & 06/03/2009 & & HCJ-148-09 \\
\hline
\end{tabular}


Table A-2. (continued).

\begin{tabular}{|c|c|c|c|c|c|c|c|c|c|c|c|}
\hline $\begin{array}{c}\text { Field Sample } \\
\text { Number }\end{array}$ & Location & Depth & Compound & $\begin{array}{c}\text { Sample } \\
\text { Result }\end{array}$ & $\begin{array}{l}\text { Sample } \\
\text { Error }\end{array}$ & $\begin{array}{c}\text { Result } \\
\text { Qualifier }\end{array}$ & $\begin{array}{c}\text { Validation } \\
\text { Flag }\end{array}$ & $\begin{array}{c}\text { Sample } \\
\text { Units }\end{array}$ & $\begin{array}{c}\text { Date Sample } \\
\text { Collected }\end{array}$ & MDA & L\&V Report Number \\
\hline GWA03001VL & USGS-001 & 588 & Dibromochloromethane & 1 & & $U$ & & UG/L & $06 / 03 / 2009$ & & HCJ-148-09 \\
\hline GWA03001VL & USGS-001 & 588 & 1,2-Dibromoethane & 1 & & U & & UG/L & $06 / 03 / 2009$ & & HCJ-148-09 \\
\hline GWA03001VL & USGS-001 & 588 & Chlorobenzene & 1 & & $\mathrm{U}$ & & UG/L & 06/03/2009 & & HCJ-148-09 \\
\hline GWA03001VL & USGS-001 & 588 & Ethylbenzene & 1 & & $\mathrm{U}$ & & UG/L & $06 / 03 / 2009$ & & HCJ-148-09 \\
\hline GWA03001VL & USGS-001 & 588 & Styrene & 1 & & U & & UG/L & $06 / 03 / 2009$ & & HCJ-148-09 \\
\hline GWA03001VL & USGS-001 & 588 & Bromoform & 1 & & $\mathrm{U}$ & & UG/L & 06/03/2009 & & HCJ-148-09 \\
\hline GWA03001VL & USGS-001 & 588 & 1,1,2,2-Tetrachloroethane & 1 & & $\mathrm{U}$ & & UG/L & $06 / 03 / 2009$ & & HCJ-148-09 \\
\hline GWA03001VL & USGS-001 & 588 & Isopropylbenzene & 1 & & U & & UG/L & $06 / 03 / 2009$ & & HCJ-148-09 \\
\hline GWA03001VL & USGS-001 & 588 & 1,3-Dichlorobenzene & 1 & & U & & UG/L & $06 / 03 / 2009$ & & HCJ-148-09 \\
\hline GWA03001VL & USGS-001 & 588 & 1,4-Dichlorobenzene & 1 & & $\mathrm{U}$ & & UG/L & $06 / 03 / 2009$ & & HCJ-148-09 \\
\hline GWA03001VL & USGS-001 & 588 & 1,2-Dibromo-3-chloropropane & 1 & & $\mathrm{U}$ & & UG/L & $06 / 03 / 2009$ & & HCJ-148-09 \\
\hline GWA03001VL & USGS-001 & 588 & Trichlorotrifluoroethane & 5 & & U & & UG/L & $06 / 03 / 2009$ & & HCJ-148-09 \\
\hline GWA03001VL & USGS-001 & 588 & Xylene (Total) & 1 & & U & & UG/L & $06 / 03 / 2009$ & & HCJ-148-09 \\
\hline GWA03001VL & USGS-001 & 588 & 1,2,4-Trichlorobenzene & 1 & & $\mathrm{u}$ & & UG/L & $06 / 03 / 2009$ & & HCJ-148-09 \\
\hline GWA03001VL & USGS-001 & 588 & 1,2-Dichlorobenzene & 1 & & U & & UG/L & $06 / 03 / 2009$ & & HCJ-148-09 \\
\hline GWA03001R8 & USGS-001 & 588 & Tritium & $-6.04 \mathrm{E}+01$ & $8.61 \mathrm{E}+01$ & & $U$ & $\mathrm{PCl} / \mathrm{L}$ & $06 / 03 / 2009$ & $3.06 \mathrm{E}+02$ & BAM-119-09 \\
\hline GWA03001RH & USGS-001 & 588 & Gross Alpha & $1.30 \mathrm{E}+00$ & 5.76E-01 & & UJ & $\mathrm{PCl} / \mathrm{L}$ & 06/03/2009 & $1.44 \mathrm{E}+00$ & BAM-120-09 \\
\hline GWA03001RH & USGS-001 & 588 & Gross Beta & $4.01 \mathrm{E}+00$ & 7.73E-01 & & & $\mathrm{PCl} / \mathrm{L}$ & $06 / 03 / 2009$ & $1.48 \mathrm{E}+00$ & BAM-120-09 \\
\hline GWA03001RH & USGS-001 & 588 & Strontium-90 & $1.98 \mathrm{E}-01$ & 9.95E-02 & & $\mathrm{U}$ & $\mathrm{PCl} / \mathrm{L}$ & 06/03/2009 & $3.18 \mathrm{E}-01$ & BAM-120-09 \\
\hline GWA03001RH & USGS-001 & 588 & Technetium-99 & $-2.50 \mathrm{E}-01$ & $1.67 \mathrm{E}+00$ & & U & $\mathrm{PCl} / \mathrm{L}$ & $06 / 03 / 2009$ & $5.84 \mathrm{E}+00$ & BAM-120-09 \\
\hline GWA03001UX & USGS-001 & 588 & lodine-129 & $-2.12 \mathrm{E}-02$ & 4.04E-02 & & $\mathrm{U}$ & $\mathrm{PCl} / \mathrm{L}$ & 06/03/2009 & 1.31E-01 & BAM-118-09 \\
\hline GWA04501A1 & USGS-002 & 659 & Alkalinity, Total as $\mathrm{CaCO} 3$ & 134 & & & & $M G / L$ & $06 / 22 / 2009$ & & HCJ-156-09 \\
\hline GWA04501AN & USGS-002 & 659 & Bromide & 0 & & U & & $M G / L$ & $06 / 22 / 2009$ & & HCJ-158-09 \\
\hline GWA04501AN & USGS-002 & 659 & Chloride & 16.6 & & & & $M G / L$ & $06 / 22 / 2009$ & & HCJ-158-09 \\
\hline GWA04501AN & USGS-002 & 659 & Fluoride & 0.666 & & & & $M G / L$ & $06 / 22 / 2009$ & & HCJ-158-09 \\
\hline GWA04501AN & USGS-002 & 659 & Sulfate & 15.2 & & & & $M G / L$ & $06 / 22 / 2009$ & & HCJ-158-09 \\
\hline GWA04501N2 & USGS-002 & 659 & Nitrogen, Nitrate/Nitrite & 1.77 & & & & MG/L & $06 / 22 / 2009$ & & HCJ-154-09 \\
\hline GWA045012X & USGS-002 & 659 & Aluminum & 15 & & $\mathrm{U}$ & & UG/L & $06 / 22 / 2009$ & & HCJ-160-09 \\
\hline GWA045012X & USGS-002 & 659 & Antimony & 1 & & U & & UG/L & $06 / 22 / 2009$ & & HCJ-160-09 \\
\hline GWA045012X & USGS-002 & 659 & Arsenic & 4.82 & & B & $\mathrm{U}$ & UG/L & $06 / 22 / 2009$ & & HCJ-160-09 \\
\hline GWA045012X & USGS-002 & 659 & Barium & 35.2 & & B & & UG/L & $06 / 22 / 2009$ & & HCJ-160-09 \\
\hline GWA045012X & USGS-002 & 659 & Beryllium & 0.1 & & $\mathrm{U}$ & & UG/L & $06 / 22 / 2009$ & & HCJ-160-09 \\
\hline GWA045012X & USGS-002 & 659 & Cadmium & 0.11 & & $\mathrm{U}$ & & UG/L & $06 / 22 / 2009$ & & HCJ-160-09 \\
\hline GWA045012X & USGS-002 & 659 & Calcium & 35200 & & & & UG/L & $06 / 22 / 2009$ & & HCJ-160-09 \\
\hline GWA045012X & USGS-002 & 659 & Chromium & 2 & & $\mathrm{u}$ & & UG/L & $06 / 22 / 2009$ & & HCJ-160-09 \\
\hline GWA045012X & USGS-002 & 659 & Cobalt & 0.1 & & U & & UG/L & $06 / 22 / 2009$ & & HCJ-160-09 \\
\hline GWA045012X & USGS-002 & 659 & Copper & 0.973 & & B & U & UG/L & $06 / 22 / 2009$ & & HCJ-160-09 \\
\hline GWA045012X & USGS-002 & 659 & Iron & 117 & & & & UG/L & $06 / 22 / 2009$ & & HCJ-160-09 \\
\hline GWA045012X & USGS-002 & 659 & Lead & 0.5 & & $\mathrm{U}$ & & UG/L & $06 / 22 / 2009$ & & HCJ-160-09 \\
\hline GWA045012X & USGS-002 & 659 & Magnesium & 10900 & & & & UG/L & $06 / 22 / 2009$ & & HCJ-160-09 \\
\hline GWA045012X & USGS-002 & 659 & Manganese & 1.56 & & B & & UG/L & $06 / 22 / 2009$ & & HCJ-160-09 \\
\hline GWA045012X & USGS-002 & 659 & Mercury & 0.066 & & U & & UG/L & $06 / 22 / 2009$ & & HCJ-160-09 \\
\hline GWA045012X & USGS-002 & 659 & Nickel & 0.863 & & B & & UG/L & $06 / 22 / 2009$ & & HCJ-160-09 \\
\hline GWA045012X & USGS-002 & 659 & Potassium & 3130 & & B & & UG/L & $06 / 22 / 2009$ & & HCJ-160-09 \\
\hline
\end{tabular}


Table A-2. (continued).

\begin{tabular}{|c|c|c|c|c|c|c|c|c|c|c|c|}
\hline $\begin{array}{c}\text { Field Sample } \\
\text { Number }\end{array}$ & Location & Depth & Compound & $\begin{array}{c}\text { Sample } \\
\text { Result }\end{array}$ & $\begin{array}{c}\text { Sample } \\
\text { Error }\end{array}$ & $\begin{array}{c}\text { Result } \\
\text { Qualifier }\end{array}$ & $\begin{array}{c}\text { Validation } \\
\text { Flag }\end{array}$ & $\begin{array}{c}\text { Sample } \\
\text { Units }\end{array}$ & $\begin{array}{c}\text { Date Sample } \\
\text { Collected }\end{array}$ & MDA & L\&V Report Number \\
\hline GWA045012X & USGS-002 & 659 & Selenium & 1 & & $U$ & & UG/L & $06 / 22 / 2009$ & & HCJ-160-09 \\
\hline GWA045012X & USGS-002 & 659 & Silver & 0.2 & & U & & UG/L & 06/22/2009 & & HCJ-160-09 \\
\hline GWA045012X & USGS-002 & 659 & Sodium & 15400 & & & & UG/L & 06/22/2009 & & HCJ-160-09 \\
\hline GWA045012X & USGS-002 & 659 & Strontium & 142 & & & & UG/L & 06/22/2009 & & HCJ-160-09 \\
\hline GWA045012X & USGS-002 & 659 & Thallium & 0.3 & & U & & UG/L & 06/22/2009 & & HCJ-160-09 \\
\hline GWA045012X & USGS-002 & 659 & Uranium & 1.93 & & B & & UG/L & 06/22/2009 & & HCJ-160-09 \\
\hline GWA045012X & USGS-002 & 659 & Vanadium & 3 & & $\mathrm{U}$ & & UG/L & 06/22/2009 & & HCJ-160-09 \\
\hline GWA045012X & USGS-002 & 659 & Zinc & 3 & & U & & UG/L & 06/22/2009 & & HCJ-160-09 \\
\hline GWA04501VL & USGS-002 & 659 & Dichlorodifluoromethane & 1 & & U & & UG/L & 06/22/2009 & & HCJ-153-09 \\
\hline GWA04501VL & USGS-002 & 659 & Chloromethane & 1 & & $\mathrm{U}$ & & UG/L & 06/22/2009 & & HCJ-153-09 \\
\hline GWA04501VL & USGS-002 & 659 & Vinyl Chloride & 1 & & $\mathrm{U}$ & & UG/L & 06/22/2009 & & HCJ-153-09 \\
\hline GWA04501VL & USGS-002 & 659 & Bromomethane & 1 & & $U$ & & UG/L & 06/22/2009 & & HCJ-153-09 \\
\hline GWA04501VL & USGS-002 & 659 & Chloroethane & 1 & & $u$ & & UG/L & 06/22/2009 & & HCJ-153-09 \\
\hline GWA04501VL & USGS-002 & 659 & Trichlorofluoromethane & 1 & & $\mathrm{U}$ & & UG/L & 06/22/2009 & & HCJ-153-09 \\
\hline GWA04501VL & USGS-002 & 659 & Acetone & 5 & & U & & UG/L & 06/22/2009 & & HCJ-153-09 \\
\hline GWA04501VL & USGS-002 & 659 & 1,1-Dichloroethene & 1 & & U & & UG/L & 06/22/2009 & & HCJ-153-09 \\
\hline GWA04501VL & USGS-002 & 659 & Methyl acetate & 5 & & $\mathrm{U}$ & & UG/L & $06 / 22 / 2009$ & & HCJ-153-09 \\
\hline GWA04501VL & USGS-002 & 659 & Methylene Chloride & 5 & & $\mathrm{U}$ & & UG/L & 06/22/2009 & & HCJ-153-09 \\
\hline GWA04501VL & USGS-002 & 659 & Carbon disulfide & 5 & & U & & UG/L & 06/22/2009 & & HCJ-153-09 \\
\hline GWA04501VL & USGS-002 & 659 & Tert-butyl methyl ether & 1 & & $\mathrm{U}$ & & UG/L & 06/22/2009 & & HCJ-153-09 \\
\hline GWA04501VL & USGS-002 & 659 & trans-1,2-Dichloroethylene & 1 & & $\mathrm{U}$ & & UG/L & 06/22/2009 & & HCJ-153-09 \\
\hline GWA04501VL & USGS-002 & 659 & 1,1-Dichloroethane & 1 & & $\mathrm{U}$ & & UG/L & $06 / 22 / 2009$ & & HCJ-153-09 \\
\hline GWA04501VL & USGS-002 & 659 & 2-Butanone & 5 & & U & & UG/L & 06/22/2009 & & HCJ-153-09 \\
\hline GWA04501VL & USGS-002 & 659 & cis-1,2-Dichloroethylene & 1 & & $u$ & & UG/L & $06 / 22 / 2009$ & & HCJ-153-09 \\
\hline GWA04501VL & USGS-002 & 659 & Chloroform & 1 & & $\mathrm{U}$ & & UG/L & 06/22/2009 & & HCJ-153-09 \\
\hline GWA04501VL & USGS-002 & 659 & 1,1,1-Trichloroethane & 1 & & U & & UG/L & 06/22/2009 & & HCJ-153-09 \\
\hline GWA04501VL & USGS-002 & 659 & Cyclohexane & 1 & & $\mathrm{U}$ & & UG/L & $06 / 22 / 2009$ & & HCJ-153-09 \\
\hline GWA04501VL & USGS-002 & 659 & Carbon tetrachloride & 1 & & $\mathrm{u}$ & & UG/L & $06 / 22 / 2009$ & & HCJ-153-09 \\
\hline GWA04501VL & USGS-002 & 659 & 1,2-Dichloroethane & 1 & & $\mathrm{U}$ & & UG/L & 06/22/2009 & & HCJ-153-09 \\
\hline GWA04501VL & USGS-002 & 659 & Benzene & 1 & & U & & UG/L & 06/22/2009 & & HCJ-153-09 \\
\hline GWA04501VL & USGS-002 & 659 & Trichloroethylene & 1 & & $\mathrm{U}$ & & UG/L & $06 / 22 / 2009$ & & HCJ-153-09 \\
\hline GWA04501VL & USGS-002 & 659 & 1,2-Dichloropropane & 1 & & $\mathrm{u}$ & & UG/L & 06/22/2009 & & HCJ-153-09 \\
\hline GWA04501VL & USGS-002 & 659 & Methyl cyclohexane & 1 & & $\mathrm{U}$ & & UG/L & 06/22/2009 & & HCJ-153-09 \\
\hline GWA04501VL & USGS-002 & 659 & Bromodichloromethane & 1 & & $U$ & & UG/L & 06/22/2009 & & HCJ-153-09 \\
\hline GWA04501VL & USGS-002 & 659 & 4-Methyl-2-pentanone & 5 & & $\mathrm{u}$ & & UG/L & $06 / 22 / 2009$ & & HCJ-153-09 \\
\hline GWA04501VL & USGS-002 & 659 & cis-1,3-Dichloropropylene & 1 & & $\mathrm{U}$ & & UG/L & 06/22/2009 & & HCJ-153-09 \\
\hline GWA04501VL & USGS-002 & 659 & Toluene & 1 & & U & & UG/L & 06/22/2009 & & HCJ-153-09 \\
\hline GWA04501VL & USGS-002 & 659 & trans-1,3-Dichloropropylene & 1 & & $u$ & & UG/L & 06/22/2009 & & HCJ-153-09 \\
\hline GWA04501VL & USGS-002 & 659 & 1,1,2-Trichloroethane & 1 & & $\mathrm{U}$ & & UG/L & 06/22/2009 & & HCJ-153-09 \\
\hline GWA04501VL & USGS-002 & 659 & 2-Hexanone & 5 & & $\mathrm{U}$ & & UG/L & 06/22/2009 & & HCJ-153-09 \\
\hline GWA04501VL & USGS-002 & 659 & Tetrachloroethylene & 1 & & $\mathrm{U}$ & & UG/L & $06 / 22 / 2009$ & & HCJ-153-09 \\
\hline GWA04501VL & USGS-002 & 659 & Dibromochloromethane & 1 & & $\mathrm{U}$ & & UG/L & $06 / 22 / 2009$ & & HCJ-153-09 \\
\hline GWA04501VL & USGS-002 & 659 & 1,2-Dibromoethane & 1 & & $u$ & & UG/L & 06/22/2009 & & HCJ-153-09 \\
\hline GWA04501VL & USGS-002 & 659 & Chlorobenzene & 1 & & U & & UG/L & 06/22/2009 & & HCJ-153-09 \\
\hline
\end{tabular}


Table A-2. (continued).

\begin{tabular}{|c|c|c|c|c|c|c|c|c|c|c|c|}
\hline $\begin{array}{c}\text { Field Sample } \\
\text { Number }\end{array}$ & Location & Depth & Compound & $\begin{array}{c}\text { Sample } \\
\text { Result }\end{array}$ & $\begin{array}{l}\text { Sample } \\
\text { Error }\end{array}$ & $\begin{array}{c}\text { Result } \\
\text { Qualifier }\end{array}$ & $\begin{array}{c}\text { Validation } \\
\text { Flag }\end{array}$ & $\begin{array}{c}\text { Sample } \\
\text { Units }\end{array}$ & $\begin{array}{c}\text { Date Sample } \\
\text { Collected }\end{array}$ & MDA & L\&V Report Number \\
\hline GWA04501VL & USGS-002 & 659 & Ethylbenzene & 1 & & $U$ & & UG/L & $06 / 22 / 2009$ & & HCJ-153-09 \\
\hline GWA04501VL & USGS-002 & 659 & Styrene & 1 & & U & & UG/L & $06 / 22 / 2009$ & & HCJ-153-09 \\
\hline GWA04501VL & USGS-002 & 659 & Bromoform & 1 & & $\mathrm{u}$ & & UG/L & $06 / 22 / 2009$ & & HCJ-153-09 \\
\hline GWA04501VL & USGS-002 & 659 & 1,1,2,2-Tetrachloroethane & 1 & & $\mathrm{U}$ & & UG/L & $06 / 22 / 2009$ & & HCJ-153-09 \\
\hline GWA04501VL & USGS-002 & 659 & Isopropylbenzene & 1 & & U & & UG/L & $06 / 22 / 2009$ & & HCJ-153-09 \\
\hline GWA04501VL & USGS-002 & 659 & 1,3-Dichlorobenzene & 1 & & U & & UG/L & $06 / 22 / 2009$ & & HCJ-153-09 \\
\hline GWA04501VL & USGS-002 & 659 & 1,4-Dichlorobenzene & 1 & & $\mathrm{U}$ & & UG/L & $06 / 22 / 2009$ & & HCJ-153-09 \\
\hline GWA04501VL & USGS-002 & 659 & 1,2-Dibromo-3-chloropropane & 1 & & U & & UG/L & $06 / 22 / 2009$ & & HCJ-153-09 \\
\hline GWA04501VL & USGS-002 & 659 & Trichlorotrifluoroethane & 5 & & U & & UG/L & $06 / 22 / 2009$ & & HCJ-153-09 \\
\hline GWA04501VL & USGS-002 & 659 & Xylene (Total) & 1 & & $\mathrm{u}$ & & UG/L & $06 / 22 / 2009$ & & HCJ-153-09 \\
\hline GWA04501VL & USGS-002 & 659 & 1,2,4-Trichlorobenzene & 1 & & $\mathrm{U}$ & & UG/L & $06 / 22 / 2009$ & & HCJ-153-09 \\
\hline GWA04501VL & USGS-002 & 659 & 1,2-Dichlorobenzene & 1 & & $U$ & & UG/L & $06 / 22 / 2009$ & & HCJ-153-09 \\
\hline GWA04501VL & USGS-002 & 659 & Unknown Siloxane & 5.5 & & $\mathrm{~J}$ & & UG/L & $06 / 22 / 2009$ & & \\
\hline GWA04501R8 & USGS-002 & 659 & Tritium & $-6.38 \mathrm{E}+00$ & $1.06 \mathrm{E}+02$ & & $\mathrm{U}$ & $\mathrm{PCl} / \mathrm{L}$ & $06 / 22 / 2009$ & $3.82 \mathrm{E}+02$ & BAM-119-09 \\
\hline GWA04501RH & USGS-002 & 659 & Gross Alpha & $1.36 \mathrm{E}+00$ & $6.76 \mathrm{E}-01$ & & UJ & $\mathrm{PCl} / \mathrm{L}$ & $06 / 22 / 2009$ & $1.84 \mathrm{E}+00$ & BAM-120-09 \\
\hline GWA04501RH & USGS-002 & 659 & Gross Beta & $3.95 \mathrm{E}+00$ & $1.09 \mathrm{E}+00$ & & & $\mathrm{PCI} / \mathrm{L}$ & $06 / 22 / 2009$ & $3.08 \mathrm{E}+00$ & BAM-120-09 \\
\hline GWA04501RH & USGS-002 & 659 & Strontium-90 & 1.93E-02 & 1.29E-01 & & $\mathrm{U}$ & $\mathrm{PCl} / \mathrm{L}$ & $06 / 22 / 2009$ & 4.55E-01 & BAM-120-09 \\
\hline GWA04501RH & USGS-002 & 659 & Technetium-99 & $-1.97 E+00$ & $1.59 \mathrm{E}+00$ & & $\mathrm{U}$ & $\mathrm{PCl} / \mathrm{L}$ & $06 / 22 / 2009$ & $5.76 \mathrm{E}+00$ & BAM-120-09 \\
\hline GWA04501UX & USGS-002 & 659 & lodine-129 & $-2.85 \mathrm{E}-02$ & $2.73 \mathrm{E}-02$ & & $\mathrm{U}$ & $\mathrm{PCl} / \mathrm{L}$ & $06 / 22 / 2009$ & 8.79E-02 & BAM-118-09 \\
\hline GWA03101A1 & USGS-009 & 607 & Alkalinity, Total as $\mathrm{CaCO} 3$ & 135 & & & & $M G / L$ & $06 / 17 / 2009$ & & HCJ-156-09 \\
\hline GWA03101AN & USGS-009 & 607 & Bromide & 0 & & $\mathrm{U}$ & & $M G / L$ & $06 / 17 / 2009$ & & HCJ-158-09 \\
\hline GWA03101AN & USGS-009 & 607 & Chloride & 15.4 & & & & $M G / L$ & $06 / 17 / 2009$ & & HCJ-158-09 \\
\hline GWA03101AN & USGS-009 & 607 & Fluoride & 0.229 & & $\mathrm{~J}$ & & $M G / L$ & $06 / 17 / 2009$ & & HCJ-158-09 \\
\hline GWA03101AN & USGS-009 & 607 & Sulfate & 24.5 & & & & $M G / L$ & $06 / 17 / 2009$ & & HCJ-158-09 \\
\hline GWA03101N2 & USGS-009 & 607 & Nitrogen, Nitrate/Nitrite & 0.705 & & & & $M G / L$ & $06 / 17 / 2009$ & & HCJ-154-09 \\
\hline GWA031012X & USGS-009 & 607 & Aluminum & 15 & & U & & UG/L & $06 / 17 / 2009$ & & HCJ-160-09 \\
\hline GWA031012X & USGS-009 & 607 & Antimony & 1 & & $u$ & & UG/L & $06 / 17 / 2009$ & & HCJ-160-09 \\
\hline GWA031012X & USGS-009 & 607 & Arsenic & 1.6 & & $\mathrm{U}$ & & UG/L & $06 / 17 / 2009$ & & HCJ-160-09 \\
\hline GWA031012X & USGS-009 & 607 & Barium & 36.1 & & B & & UG/L & $06 / 17 / 2009$ & & HCJ-160-09 \\
\hline GWA031012X & USGS-009 & 607 & Beryllium & 0.1 & & U & & UG/L & $06 / 17 / 2009$ & & HCJ-160-09 \\
\hline GWA031012X & USGS-009 & 607 & Cadmium & 0.11 & & $u$ & & UG/L & $06 / 17 / 2009$ & & HCJ-160-09 \\
\hline GWA031012X & USGS-009 & 607 & Calcium & 38400 & & & & UG/L & $06 / 17 / 2009$ & & HCJ-160-09 \\
\hline GWA031012X & USGS-009 & 607 & Chromium & 3.46 & & B & & UG/L & $06 / 17 / 2009$ & & HCJ-160-09 \\
\hline GWA031012X & USGS-009 & 607 & Cobalt & 0.1 & & U & & UG/L & $06 / 17 / 2009$ & & HCJ-160-09 \\
\hline GWA031012X & USGS-009 & 607 & Copper & 0.557 & & B & $\mathrm{u}$ & UG/L & $06 / 17 / 2009$ & & HCJ-160-09 \\
\hline GWA031012X & USGS-009 & 607 & Iron & 157 & & & & UG/L & $06 / 17 / 2009$ & & HCJ-160-09 \\
\hline GWA031012X & USGS-009 & 607 & Lead & 0.5 & & U & & UG/L & $06 / 17 / 2009$ & & HCJ-160-09 \\
\hline GWA031012X & USGS-009 & 607 & Magnesium & 13300 & & & & UG/L & $06 / 17 / 2009$ & & HCJ-160-09 \\
\hline GWA031012X & USGS-009 & 607 & Manganese & 6.15 & & B & & UG/L & $06 / 17 / 2009$ & & HCJ-160-09 \\
\hline GWA031012X & USGS-009 & 607 & Mercury & 0.066 & & $U$ & & UG/L & $06 / 17 / 2009$ & & HCJ-160-09 \\
\hline GWA031012X & USGS-009 & 607 & Nickel & 0.81 & & B & & UG/L & $06 / 17 / 2009$ & & HCJ-160-09 \\
\hline GWA031012X & USGS-009 & 607 & Potassium & 2840 & & B & & UG/L & $06 / 17 / 2009$ & & HCJ-160-09 \\
\hline GWA031012X & USGS-009 & 607 & Selenium & 1.16 & & B & & UG/L & $06 / 17 / 2009$ & & HCJ-160-09 \\
\hline GWA031012X & USGS-009 & 607 & Silver & 0.2 & & $U$ & & UG/L & $06 / 17 / 2009$ & & HCJ-160-09 \\
\hline
\end{tabular}


Table A-2. (continued).

\begin{tabular}{|c|c|c|c|c|c|c|c|c|c|c|c|}
\hline $\begin{array}{c}\text { Field Sample } \\
\text { Number }\end{array}$ & Location & Depth & Compound & $\begin{array}{c}\text { Sample } \\
\text { Result }\end{array}$ & $\begin{array}{l}\text { Sample } \\
\text { Error }\end{array}$ & $\begin{array}{c}\text { Result } \\
\text { Qualifier }\end{array}$ & $\begin{array}{c}\text { Validation } \\
\text { Flag }\end{array}$ & $\begin{array}{c}\text { Sample } \\
\text { Units }\end{array}$ & $\begin{array}{c}\text { Date Sample } \\
\text { Collected }\end{array}$ & MDA & L\&V Report Number \\
\hline GWA031012X & USGS-009 & 607 & Sodium & 9910 & & & & UG/L & $06 / 17 / 2009$ & & HCJ-160-09 \\
\hline GWA031012X & USGS-009 & 607 & Strontium & 203 & & & & UG/L & 06/17/2009 & & HCJ-160-09 \\
\hline GWA031012X & USGS-009 & 607 & Thallium & 0.3 & & $\mathrm{U}$ & & UG/L & 06/17/2009 & & HCJ-160-09 \\
\hline GWA031012X & USGS-009 & 607 & Uranium & 1.94 & & B & & UG/L & 06/17/2009 & & HCJ-160-09 \\
\hline GWA031012X & USGS-009 & 607 & Vanadium & 3 & & $\mathrm{U}$ & & UG/L & 06/17/2009 & & HCJ-160-09 \\
\hline GWA031012X & USGS-009 & 607 & Zinc & 3 & & $\mathrm{U}$ & & UG/L & 06/17/2009 & & HCJ-160-09 \\
\hline GWA03101VL & USGS-009 & 607 & Dichlorodifluoromethane & 1 & & U & & UG/L & 06/17/2009 & & HCJ-153-09 \\
\hline GWA03101VL & USGS-009 & 607 & Chloromethane & 1 & & U & & UG/L & 06/17/2009 & & HCJ-153-09 \\
\hline GWA03101VL & USGS-009 & 607 & Vinyl Chloride & 1 & & $\mathrm{U}$ & & UG/L & 06/17/2009 & & HCJ-153-09 \\
\hline GWA03101VL & USGS-009 & 607 & Bromomethane & 1 & & $\mathrm{U}$ & & UG/L & 06/17/2009 & & HCJ-153-09 \\
\hline GWA03101VL & USGS-009 & 607 & Chloroethane & 1 & & $\mathrm{U}$ & & UG/L & 06/17/2009 & & HCJ-153-09 \\
\hline GWA03101VL & USGS-009 & 607 & Trichlorofluoromethane & 1 & & $\mathrm{U}$ & & UG/L & 06/17/2009 & & HCJ-153-09 \\
\hline GWA03101VL & USGS-009 & 607 & Acetone & 5 & & $\mathrm{U}$ & & UG/L & 06/17/2009 & & HCJ-153-09 \\
\hline GWA03101VL & USGS-009 & 607 & 1,1-Dichloroethene & 1 & & $\mathrm{U}$ & & UG/L & 06/17/2009 & & HCJ-153-09 \\
\hline GWA03101VL & USGS-009 & 607 & Methyl acetate & 5 & & $\mathrm{U}$ & & UG/L & 06/17/2009 & & HCJ-153-09 \\
\hline GWA03101VL & USGS-009 & 607 & Methylene Chloride & 5 & & $\mathrm{U}$ & & UG/L & 06/17/2009 & & HCJ-153-09 \\
\hline GWA03101VL & USGS-009 & 607 & Carbon disulfide & 5 & & $\mathrm{U}$ & & UG/L & 06/17/2009 & & HCJ-153-09 \\
\hline GWA03101VL & USGS-009 & 607 & Tert-butyl methyl ether & 1 & & $U$ & & UG/L & 06/17/2009 & & HCJ-153-09 \\
\hline GWA03101VL & USGS-009 & 607 & trans-1,2-Dichloroethylene & 1 & & $\mathrm{U}$ & & UG/L & 06/17/2009 & & HCJ-153-09 \\
\hline GWA03101VL & USGS-009 & 607 & 1,1-Dichloroethane & 1 & & U & & UG/L & 06/17/2009 & & HCJ-153-09 \\
\hline GWA03101VL & USGS-009 & 607 & 2-Butanone & 5 & & $\mathrm{U}$ & & UG/L & 06/17/2009 & & HCJ-153-09 \\
\hline GWA03101VL & USGS-009 & 607 & cis-1,2-Dichloroethylene & 1 & & $\mathrm{U}$ & & UG/L & 06/17/2009 & & HCJ-153-09 \\
\hline GWA03101VL & USGS-009 & 607 & Chloroform & 1 & & $\mathrm{U}$ & & UG/L & 06/17/2009 & & HCJ-153-09 \\
\hline GWA03101VL & USGS-009 & 607 & 1,1,1-Trichloroethane & 1 & & $\mathrm{U}$ & & UG/L & 06/17/2009 & & HCJ-153-09 \\
\hline GWA03101VL & USGS-009 & 607 & Cyclohexane & 1 & & $\mathrm{U}$ & & UG/L & 06/17/2009 & & HCJ-153-09 \\
\hline GWA03101VL & USGS-009 & 607 & Carbon tetrachloride & 1 & & $\mathrm{U}$ & & UG/L & 06/17/2009 & & HCJ-153-09 \\
\hline GWA03101VL & USGS-009 & 607 & 1,2-Dichloroethane & 1 & & $\mathrm{U}$ & & UG/L & 06/17/2009 & & HCJ-153-09 \\
\hline GWA03101VL & USGS-009 & 607 & Benzene & 1 & & $\mathrm{U}$ & & UG/L & 06/17/2009 & & HCJ-153-09 \\
\hline GWA03101VL & USGS-009 & 607 & Trichloroethylene & 1 & & $\mathrm{U}$ & & UG/L & 06/17/2009 & & HCJ-153-09 \\
\hline GWA03101VL & USGS-009 & 607 & 1,2-Dichloropropane & 1 & & $\mathrm{U}$ & & UG/L & 06/17/2009 & & HCJ-153-09 \\
\hline GWA03101VL & USGS-009 & 607 & Methyl cyclohexane & 1 & & $\mathrm{U}$ & & UG/L & 06/17/2009 & & HCJ-153-09 \\
\hline GWA03101VL & USGS-009 & 607 & Bromodichloromethane & 1 & & $\mathrm{U}$ & & UG/L & 06/17/2009 & & HCJ-153-09 \\
\hline GWA03101VL & USGS-009 & 607 & 4-Methyl-2-pentanone & 5 & & $\mathrm{U}$ & & UG/L & 06/17/2009 & & HCJ-153-09 \\
\hline GWA03101VL & USGS-009 & 607 & cis-1,3-Dichloropropylene & 1 & & $U$ & & UG/L & 06/17/2009 & & HCJ-153-09 \\
\hline GWA03101VL & USGS-009 & 607 & Toluene & 1 & & $\mathrm{U}$ & & UG/L & 06/17/2009 & & HCJ-153-09 \\
\hline GWA03101VL & USGS-009 & 607 & trans-1,3-Dichloropropylene & 1 & & $\mathrm{U}$ & & UG/L & 06/17/2009 & & HCJ-153-09 \\
\hline GWA03101VL & USGS-009 & 607 & 1,1,2-Trichloroethane & 1 & & $\mathrm{U}$ & & UG/L & 06/17/2009 & & HCJ-153-09 \\
\hline GWA03101VL & USGS-009 & 607 & 2-Hexanone & 5 & & $U$ & & UG/L & 06/17/2009 & & HCJ-153-09 \\
\hline GWA03101VL & USGS-009 & 607 & Tetrachloroethylene & 1 & & $\mathrm{U}$ & & UG/L & 06/17/2009 & & HCJ-153-09 \\
\hline GWA03101VL & USGS-009 & 607 & Dibromochloromethane & 1 & & $\mathrm{U}$ & & UG/L & 06/17/2009 & & HCJ-153-09 \\
\hline GWA03101VL & USGS-009 & 607 & 1,2-Dibromoethane & 1 & & $\mathrm{U}$ & & UG/L & 06/17/2009 & & HCJ-153-09 \\
\hline GWA03101VL & USGS-009 & 607 & Chlorobenzene & 1 & & $\mathrm{U}$ & & UG/L & 06/17/2009 & & HCJ-153-09 \\
\hline GWA03101VL & USGS-009 & 607 & Ethylbenzene & 1 & & $\mathrm{U}$ & & UG/L & 06/17/2009 & & HCJ-153-09 \\
\hline GWA03101VL & USGS-009 & 607 & Styrene & 1 & & $\mathrm{U}$ & & UG/L & 06/17/2009 & & HCJ-153-09 \\
\hline
\end{tabular}


Table A-2. (continued).

\begin{tabular}{|c|c|c|c|c|c|c|c|c|c|c|c|}
\hline $\begin{array}{c}\text { Field Sample } \\
\text { Number }\end{array}$ & Location & Depth & Compound & $\begin{array}{l}\text { Sample } \\
\text { Result }\end{array}$ & $\begin{array}{l}\text { Sample } \\
\text { Error }\end{array}$ & $\begin{array}{c}\text { Result } \\
\text { Qualifier }\end{array}$ & $\begin{array}{c}\text { Validation } \\
\text { Flag }\end{array}$ & $\begin{array}{c}\text { Sample } \\
\text { Units }\end{array}$ & $\begin{array}{c}\text { Date Sample } \\
\text { Collected }\end{array}$ & MDA & L\&V Report Number \\
\hline GWA03101VL & USGS-009 & 607 & Bromoform & 1 & & $\bar{U}$ & & UG/L & $06 / 17 / 2009$ & & HCJ-153-09 \\
\hline GWA03101VL & USGS-009 & 607 & 1,1,2,2-Tetrachloroethane & 1 & & $\mathrm{U}$ & & UG/L & 06/17/2009 & & HCJ-153-09 \\
\hline GWA03101VL & USGS-009 & 607 & Isopropylbenzene & 1 & & $\mathrm{U}$ & & UG/L & 06/17/2009 & & HCJ-153-09 \\
\hline GWA03101VL & USGS-009 & 607 & 1,3-Dichlorobenzene & 1 & & $\mathrm{U}$ & & UG/L & 06/17/2009 & & HCJ-153-09 \\
\hline GWA03101VL & USGS-009 & 607 & 1,4-Dichlorobenzene & 1 & & $\mathrm{U}$ & & UG/L & 06/17/2009 & & HCJ-153-09 \\
\hline GWA03101VL & USGS-009 & 607 & 1,2-Dibromo-3-chloropropane & 1 & & $\mathrm{U}$ & & UG/L & 06/17/2009 & & HCJ-153-09 \\
\hline GWA03101VL & USGS-009 & 607 & Trichlorotrifluoroethane & 5 & & U & & UG/L & 06/17/2009 & & HCJ-153-09 \\
\hline GWA03101VL & USGS-009 & 607 & Xylene (Total) & 1 & & $\mathrm{U}$ & & UG/L & 06/17/2009 & & HCJ-153-09 \\
\hline GWA03101VL & USGS-009 & 607 & 1,2,4-Trichlorobenzene & 1 & & $\mathrm{U}$ & & UG/L & 06/17/2009 & & HCJ-153-09 \\
\hline GWA03101VL & USGS-009 & 607 & 1,2-Dichlorobenzene & 1 & & $\mathrm{U}$ & & UG/L & 06/17/2009 & & HCJ-153-09 \\
\hline GWA03101R8 & USGS-009 & 607 & Tritium & $4.73 E+01$ & $8.94 \mathrm{E}+01$ & & $\mathrm{U}$ & $\mathrm{PCl} / \mathrm{L}$ & 06/17/2009 & $3.05 E+02$ & BAM-119-09 \\
\hline GWA03101RH & USGS-009 & 607 & Gross Alpha & $2.40 \mathrm{E}+00$ & $9.11 \mathrm{E}-01$ & & $\mathrm{~J}$ & $\mathrm{PCl} / \mathrm{L}$ & 06/17/2009 & $2.31 \mathrm{E}+00$ & BAM-120-09 \\
\hline GWA03101RH & USGS-009 & 607 & Gross Beta & $6.42 \mathrm{E}+00$ & $1.21 \mathrm{E}+00$ & & & $\mathrm{PCI} / \mathrm{L}$ & 06/17/2009 & $2.81 \mathrm{E}+00$ & BAM-120-09 \\
\hline GWA03101RH & USGS-009 & 607 & Strontium-90 & 3.12E-01 & $1.30 \mathrm{E}-01$ & & UJ & $\mathrm{PCl} / \mathrm{L}$ & 06/17/2009 & 4.10E-01 & BAM-120-09 \\
\hline GWA03101RH & USGS-009 & 607 & Technetium-99 & $-3.90 \mathrm{E}+00$ & $1.59 \mathrm{E}+00$ & & $\mathrm{U}$ & $\mathrm{PCl} / \mathrm{L}$ & 06/17/2009 & $5.95 \mathrm{E}+00$ & BAM-120-09 \\
\hline GWA03101UX & USGS-009 & 607 & lodine-129 & $-4.88 \mathrm{E}-02$ & $3.50 \mathrm{E}-02$ & & $\mathrm{U}$ & $\mathrm{PCl} / \mathrm{L}$ & 06/17/2009 & $1.01 \mathrm{E}-01$ & BAM-118-09 \\
\hline GWA03201A1 & USGS-086 & 649 & Alkalinity, Total as $\mathrm{CaCO} 3$ & 110 & & & & MG/L & 06/16/2009 & & HCJ-156-09 \\
\hline GWA03201AN & USGS-086 & 649 & Bromide & 0 & & U & & MG/L & 06/16/2009 & & HCJ-158-09 \\
\hline GWA03201AN & USGS-086 & 649 & Chloride & 17.2 & & & & MG/L & 06/16/2009 & & HCJ-158-09 \\
\hline GWA03201AN & USGS-086 & 649 & Fluoride & 0.21 & & $\mathrm{~J}$ & & MG/L & 06/16/2009 & & HCJ-158-09 \\
\hline GWA03201AN & USGS-086 & 649 & Sulfate & 23.2 & & & & MG/L & 06/16/2009 & & HCJ-158-09 \\
\hline GWA03201N2 & USGS-086 & 649 & Nitrogen, Nitrate/Nitrite & 1.35 & & & & MG/L & 06/16/2009 & & HCJ-154-09 \\
\hline GWA032012X & USGS-086 & 649 & Aluminum & 15 & & $\mathrm{U}$ & & UG/L & 06/16/2009 & & HCJ-160-09 \\
\hline GWA032012X & USGS-086 & 649 & Antimony & 1 & & $\mathrm{U}$ & & UG/L & 06/16/2009 & & HCJ-160-09 \\
\hline GWA032012X & USGS-086 & 649 & Arsenic & 1.61 & & B & $U$ & UG/L & 06/16/2009 & & HCJ-160-09 \\
\hline GWA032012X & USGS-086 & 649 & Barium & 17.4 & & B & & UG/L & 06/16/2009 & & HCJ-160-09 \\
\hline GWA032012X & USGS-086 & 649 & Beryllium & 0.1 & & $U$ & & UG/L & 06/16/2009 & & HCJ-160-09 \\
\hline GWA032012X & USGS-086 & 649 & Cadmium & 0.11 & & U & & UG/L & 06/16/2009 & & HCJ-160-09 \\
\hline GWA032012X & USGS-086 & 649 & Calcium & 36700 & & & & UG/L & 06/16/2009 & & HCJ-160-09 \\
\hline GWA032012X & USGS-086 & 649 & Chromium & 11.5 & & & & UG/L & 06/16/2009 & & HCJ-160-09 \\
\hline GWA032012X & USGS-086 & 649 & Cobalt & 0.1 & & $\mathrm{U}$ & & UG/L & 06/16/2009 & & HCJ-160-09 \\
\hline GWA032012X & USGS-086 & 649 & Copper & 0.492 & & B & $U$ & UG/L & 06/16/2009 & & HCJ-160-09 \\
\hline GWA032012X & USGS-086 & 649 & Iron & 89.9 & & B & & UG/L & 06/16/2009 & & HCJ-160-09 \\
\hline GWA032012X & USGS-086 & 649 & Lead & 0.5 & & $U$ & & UG/L & 06/16/2009 & & HCJ-160-09 \\
\hline GWA032012X & USGS-086 & 649 & Magnesium & 9430 & & & & UG/L & 06/16/2009 & & HCJ-160-09 \\
\hline GWA032012X & USGS-086 & 649 & Manganese & 1 & & U & & UG/L & 06/16/2009 & & HCJ-160-09 \\
\hline GWA032012X & USGS-086 & 649 & Mercury & 0.066 & & $\mathrm{U}$ & & UG/L & 06/16/2009 & & HCJ-160-09 \\
\hline GWA032012X & USGS-086 & 649 & Nickel & 0.671 & & B & & UG/L & 06/16/2009 & & HCJ-160-09 \\
\hline GWA032012X & USGS-086 & 649 & Potassium & 2800 & & B & & UG/L & 06/16/2009 & & HCJ-160-09 \\
\hline GWA032012X & USGS-086 & 649 & Selenium & 1.55 & & B & & UG/L & 06/16/2009 & & HCJ-160-09 \\
\hline GWA032012X & USGS-086 & 649 & Silver & 0.2 & & $U$ & & UG/L & 06/16/2009 & & HCJ-160-09 \\
\hline GWA032012X & USGS-086 & 649 & Sodium & 10400 & & & & UG/L & 06/16/2009 & & HCJ-160-09 \\
\hline GWA032012X & USGS-086 & 649 & Strontium & 153 & & & & UG/L & 06/16/2009 & & HCJ-160-09 \\
\hline GWA032012X & USGS-086 & 649 & Thallium & 0.3 & & U & & UG/L & 06/16/2009 & & HCJ-160-09 \\
\hline
\end{tabular}


Table A-2. (continued).

\begin{tabular}{|c|c|c|c|c|c|c|c|c|c|c|c|}
\hline $\begin{array}{c}\text { Field Sample } \\
\text { Number }\end{array}$ & Location & Depth & Compound & $\begin{array}{c}\text { Sample } \\
\text { Result }\end{array}$ & $\begin{array}{c}\text { Sample } \\
\text { Error }\end{array}$ & $\begin{array}{c}\text { Result } \\
\text { Qualifier }\end{array}$ & $\begin{array}{c}\text { Validation } \\
\text { Flag }\end{array}$ & $\begin{array}{c}\text { Sample } \\
\text { Units }\end{array}$ & $\begin{array}{c}\text { Date Sample } \\
\text { Collected }\end{array}$ & MDA & L\&V Report Number \\
\hline GWA032012X & USGS-086 & 649 & Uranium & 1.33 & & $\mathrm{~B}$ & & UG/L & $06 / 16 / 2009$ & & HCJ-160-09 \\
\hline GWA032012X & USGS-086 & 649 & Vanadium & 3 & & U & & UG/L & 06/16/2009 & & HCJ-160-09 \\
\hline GWA032012X & USGS-086 & 649 & Zinc & 3 & & $\mathrm{u}$ & & UG/L & 06/16/2009 & & HCJ-160-09 \\
\hline GWA03201VL & USGS-086 & 649 & Dichlorodifluoromethane & 1 & & $\mathrm{U}$ & & UG/L & 06/16/2009 & & HCJ-153-09 \\
\hline GWA03201VL & USGS-086 & 649 & Chloromethane & 1 & & U & & UG/L & 06/16/2009 & & HCJ-153-09 \\
\hline GWA03201VL & USGS-086 & 649 & Vinyl Chloride & 1 & & $\mathrm{U}$ & & UG/L & 06/16/2009 & & HCJ-153-09 \\
\hline GWA03201VL & USGS-086 & 649 & Bromomethane & 1 & & $\mathrm{U}$ & & UG/L & 06/16/2009 & & HCJ-153-09 \\
\hline GWA03201VL & USGS-086 & 649 & Chloroethane & 1 & & U & & UG/L & 06/16/2009 & & HCJ-153-09 \\
\hline GWA03201VL & USGS-086 & 649 & Trichlorofluoromethane & 1 & & U & & UG/L & 06/16/2009 & & HCJ-153-09 \\
\hline GWA03201VL & USGS-086 & 649 & Acetone & 5 & & $\mathrm{U}$ & & UG/L & 06/16/2009 & & HCJ-153-09 \\
\hline GWA03201VL & USGS-086 & 649 & 1,1-Dichloroethene & 1 & & $\mathrm{U}$ & & UG/L & 06/16/2009 & & HCJ-153-09 \\
\hline GWA03201VL & USGS-086 & 649 & Methyl acetate & 5 & & U & & UG/L & 06/16/2009 & & HCJ-153-09 \\
\hline GWA03201VL & USGS-086 & 649 & Methylene Chloride & 5 & & $u$ & & UG/L & 06/16/2009 & & HCJ-153-09 \\
\hline GWA03201VL & USGS-086 & 649 & Carbon disulfide & 5 & & $\mathrm{u}$ & & UG/L & 06/16/2009 & & HCJ-153-09 \\
\hline GWA03201VL & USGS-086 & 649 & Tert-butyl methyl ether & 1 & & U & & UG/L & 06/16/2009 & & HCJ-153-09 \\
\hline GWA03201VL & USGS-086 & 649 & trans-1,2-Dichloroethylene & 1 & & U & & UG/L & 06/16/2009 & & HCJ-153-09 \\
\hline GWA03201VL & USGS-086 & 649 & 1,1-Dichloroethane & 1 & & $\mathrm{u}$ & & UG/L & 06/16/2009 & & HCJ-153-09 \\
\hline GWA03201VL & USGS-086 & 649 & 2-Butanone & 5 & & $\mathrm{u}$ & & UG/L & 06/16/2009 & & HCJ-153-09 \\
\hline GWA03201VL & USGS-086 & 649 & cis-1,2-Dichloroethylene & 1 & & U & & UG/L & 06/16/2009 & & HCJ-153-09 \\
\hline GWA03201VL & USGS-086 & 649 & Chloroform & 1 & & $\mathrm{U}$ & & UG/L & 06/16/2009 & & HCJ-153-09 \\
\hline GWA03201VL & USGS-086 & 649 & 1,1,1-Trichloroethane & 1 & & $\mathrm{u}$ & & UG/L & 06/16/2009 & & HCJ-153-09 \\
\hline GWA03201VL & USGS-086 & 649 & Cyclohexane & 1 & & U & & UG/L & 06/16/2009 & & HCJ-153-09 \\
\hline GWA03201VL & USGS-086 & 649 & Carbon tetrachloride & 1 & & U & & UG/L & 06/16/2009 & & HCJ-153-09 \\
\hline GWA03201VL & USGS-086 & 649 & 1,2-Dichloroethane & 1 & & U & & UG/L & 06/16/2009 & & HCJ-153-09 \\
\hline GWA03201VL & USGS-086 & 649 & Benzene & 1 & & $\mathrm{u}$ & & UG/L & $06 / 16 / 2009$ & & HCJ-153-09 \\
\hline GWA03201VL & USGS-086 & 649 & Trichloroethylene & 1 & & U & & UG/L & 06/16/2009 & & HCJ-153-09 \\
\hline GWA03201VL & USGS-086 & 649 & 1,2-Dichloropropane & 1 & & $\mathrm{U}$ & & UG/L & $06 / 16 / 2009$ & & HCJ-153-09 \\
\hline GWA03201VL & USGS-086 & 649 & Methyl cyclohexane & 1 & & $\mathrm{u}$ & & UG/L & 06/16/2009 & & HCJ-153-09 \\
\hline GWA03201VL & USGS-086 & 649 & Bromodichloromethane & 1 & & $\mathrm{U}$ & & UG/L & 06/16/2009 & & HCJ-153-09 \\
\hline GWA03201VL & USGS-086 & 649 & 4-Methyl-2-pentanone & 5 & & $\mathrm{U}$ & & UG/L & 06/16/2009 & & HCJ-153-09 \\
\hline GWA03201VL & USGS-086 & 649 & cis-1,3-Dichloropropylene & 1 & & $u$ & & UG/L & 06/16/2009 & & HCJ-153-09 \\
\hline GWA03201VL & USGS-086 & 649 & Toluene & 1 & & $\mathrm{u}$ & & UG/L & 06/16/2009 & & HCJ-153-09 \\
\hline GWA03201VL & USGS-086 & 649 & trans-1,3-Dichloropropylene & 1 & & $\mathrm{U}$ & & UG/L & 06/16/2009 & & HCJ-153-09 \\
\hline GWA03201VL & USGS-086 & 649 & 1,1,2-Trichloroethane & 1 & & $U$ & & UG/L & 06/16/2009 & & HCJ-153-09 \\
\hline GWA03201VL & USGS-086 & 649 & 2-Hexanone & 5 & & $\mathrm{u}$ & & UG/L & $06 / 16 / 2009$ & & HCJ-153-09 \\
\hline GWA03201VL & USGS-086 & 649 & Tetrachloroethylene & 1 & & $\mathrm{U}$ & & UG/L & 06/16/2009 & & HCJ-153-09 \\
\hline GWA03201VL & USGS-086 & 649 & Dibromochloromethane & 1 & & U & & UG/L & 06/16/2009 & & HCJ-153-09 \\
\hline GWA03201VL & USGS-086 & 649 & 1,2-Dibromoethane & 1 & & $u$ & & UG/L & 06/16/2009 & & HCJ-153-09 \\
\hline GWA03201VL & USGS-086 & 649 & Chlorobenzene & 1 & & $\mathrm{U}$ & & UG/L & 06/16/2009 & & HCJ-153-09 \\
\hline GWA03201VL & USGS-086 & 649 & Ethylbenzene & 1 & & $\mathrm{U}$ & & UG/L & 06/16/2009 & & HCJ-153-09 \\
\hline GWA03201VL & USGS-086 & 649 & Styrene & 1 & & $\mathrm{U}$ & & UG/L & $06 / 16 / 2009$ & & HCJ-153-09 \\
\hline GWA03201VL & USGS-086 & 649 & Bromoform & 1 & & $\mathrm{U}$ & & UG/L & 06/16/2009 & & HCJ-153-09 \\
\hline GWA03201VL & USGS-086 & 649 & 1,1,2,2-Tetrachloroethane & 1 & & $u$ & & UG/L & 06/16/2009 & & HCJ-153-09 \\
\hline GWA03201VL & USGS-086 & 649 & Isopropylbenzene & 1 & & U & & UG/L & 06/16/2009 & & HCJ-153-09 \\
\hline
\end{tabular}


Table A-2. (continued).

\begin{tabular}{|c|c|c|c|c|c|c|c|c|c|c|c|}
\hline $\begin{array}{c}\text { Field Sample } \\
\text { Number }\end{array}$ & Location & Depth & Compound & $\begin{array}{c}\text { Sample } \\
\text { Result }\end{array}$ & $\begin{array}{l}\text { Sample } \\
\text { Error }\end{array}$ & $\begin{array}{c}\text { Result } \\
\text { Qualifier }\end{array}$ & $\begin{array}{c}\text { Validation } \\
\text { Flag }\end{array}$ & $\begin{array}{c}\text { Sample } \\
\text { Units }\end{array}$ & $\begin{array}{c}\text { Date Sample } \\
\text { Collected }\end{array}$ & MDA & L\&V Report Number \\
\hline GWA03201VL & USGS-086 & 649 & 1,3-Dichlorobenzene & 1 & & $U$ & & UG/L & $06 / 16 / 2009$ & & HCJ-153-09 \\
\hline GWA03201VL & USGS-086 & 649 & 1,4-Dichlorobenzene & 1 & & U & & UG/L & $06 / 16 / 2009$ & & HCJ-153-09 \\
\hline GWA03201VL & USGS-086 & 649 & 1,2-Dibromo-3-chloropropane & 1 & & $\mathrm{u}$ & & UG/L & $06 / 16 / 2009$ & & HCJ-153-09 \\
\hline GWA03201VL & USGS-086 & 649 & Trichlorotrifluoroethane & 5 & & $\mathrm{U}$ & & UG/L & $06 / 16 / 2009$ & & HCJ-153-09 \\
\hline GWA03201VL & USGS-086 & 649 & Xylene (Total) & 1 & & U & & UG/L & $06 / 16 / 2009$ & & HCJ-153-09 \\
\hline GWA03201VL & USGS-086 & 649 & 1,2,4-Trichlorobenzene & 1 & & U & & UG/L & $06 / 16 / 2009$ & & HCJ-153-09 \\
\hline GWA03201VL & USGS-086 & 649 & 1,2-Dichlorobenzene & 1 & & $\mathrm{U}$ & & UG/L & $06 / 16 / 2009$ & & HCJ-153-09 \\
\hline GWA03201R8 & USGS-086 & 649 & Tritium & $-1.14 \mathrm{E}+02$ & $8.42 \mathrm{E}+01$ & & U & $\mathrm{PCI} / \mathrm{L}$ & $06 / 16 / 2009$ & $3.05 \mathrm{E}+02$ & BAM-119-09 \\
\hline GWA03201RH & USGS-086 & 649 & Gross Alpha & $4.22 \mathrm{E}+00$ & $8.49 \mathrm{E}-01$ & & & $\mathrm{PCl} / \mathrm{L}$ & $06 / 16 / 2009$ & $1.68 \mathrm{E}+00$ & BAM-120-09 \\
\hline GWA03201RH & USGS-086 & 649 & Gross Beta & $6.30 \mathrm{E}+00$ & 8.82E-01 & & & $\mathrm{PCl} / \mathrm{L}$ & $06 / 16 / 2009$ & $1.76 \mathrm{E}+00$ & BAM-120-09 \\
\hline GWA03201RH & USGS-086 & 649 & Strontium-90 & $2.08 \mathrm{E}-01$ & $9.89 \mathrm{E}-02$ & & UJ & $\mathrm{PCl} / \mathrm{L}$ & $06 / 16 / 2009$ & $3.15 \mathrm{E}-01$ & BAM-120-09 \\
\hline GWA03201RH & USGS-086 & 649 & Technetium-99 & $-7.86 \mathrm{E}-01$ & $1.64 \mathrm{E}+00$ & & $\mathrm{U}$ & $\mathrm{PCI} / \mathrm{L}$ & $06 / 16 / 2009$ & $5.77 \mathrm{E}+00$ & BAM-120-09 \\
\hline GWA03201UX & USGS-086 & 649 & lodine-129 & $-1.77 \mathrm{E}-02$ & $2.66 \mathrm{E}-02$ & & U & $\mathrm{PCI} / \mathrm{L}$ & $06 / 16 / 2009$ & 7.75E-02 & BAM-118-09 \\
\hline GWA04601A1 & USGS-100 & 686 & Alkalinity, Total as $\mathrm{CaCO} 3$ & 134 & & & & $M G / L$ & $06 / 23 / 2009$ & & HCJ-156-09 \\
\hline GWA04601AN & USGS-100 & 686 & Bromide & 0 & & U & & $M G / L$ & $06 / 23 / 2009$ & & HCJ-158-09 \\
\hline GWA04601AN & USGS-100 & 686 & Chloride & 16.3 & & & & $M G / L$ & $06 / 23 / 2009$ & & HCJ-158-09 \\
\hline GWA04601AN & USGS-100 & 686 & Fluoride & 0.717 & & & & $M G / L$ & $06 / 23 / 2009$ & & HCJ-158-09 \\
\hline GWA04601AN & USGS-100 & 686 & Sulfate & 17.1 & & & & $M G / L$ & $06 / 23 / 2009$ & & HCJ-158-09 \\
\hline GWA04601N2 & USGS-100 & 686 & Nitrogen, Nitrate/Nitrite & 1.95 & & & & $M G / L$ & $06 / 23 / 2009$ & & HCJ-154-09 \\
\hline GWA046012X & USGS-100 & 686 & Aluminum & 15 & & U & & UG/L & $06 / 23 / 2009$ & & HCJ-160-09 \\
\hline GWA046012X & USGS-100 & 686 & Antimony & 1 & & $\mathrm{U}$ & & UG/L & $06 / 23 / 2009$ & & HCJ-160-09 \\
\hline GWA046012X & USGS-100 & 686 & Arsenic & 3.85 & & B & U & UG/L & $06 / 23 / 2009$ & & HCJ-160-09 \\
\hline GWA046012X & USGS-100 & 686 & Barium & 37.2 & & B & & UG/L & $06 / 23 / 2009$ & & HCJ-160-09 \\
\hline GWA046012X & USGS-100 & 686 & Beryllium & 0.1 & & $U$ & & UG/L & $06 / 23 / 2009$ & & HCJ-160-09 \\
\hline GWA046012X & USGS-100 & 686 & Cadmium & 0.525 & & B & & UG/L & $06 / 23 / 2009$ & & HCJ-160-09 \\
\hline GWA046012X & USGS-100 & 686 & Calcium & 36300 & & & & UG/L & $06 / 23 / 2009$ & & HCJ-160-09 \\
\hline GWA046012X & USGS-100 & 686 & Chromium & 2 & & $u$ & & UG/L & $06 / 23 / 2009$ & & HCJ-160-09 \\
\hline GWA046012X & USGS-100 & 686 & Cobalt & 0.1 & & U & & UG/L & $06 / 23 / 2009$ & & HCJ-160-09 \\
\hline GWA046012X & USGS-100 & 686 & Copper & 0.846 & & B & $U$ & UG/L & $06 / 23 / 2009$ & & HCJ-160-09 \\
\hline GWA046012X & USGS-100 & 686 & Iron & 93.8 & & B & & UG/L & $06 / 23 / 2009$ & & HCJ-160-09 \\
\hline GWA046012X & USGS-100 & 686 & Lead & 14.1 & & & & UG/L & $06 / 23 / 2009$ & & HCJ-160-09 \\
\hline GWA046012X & USGS-100 & 686 & Magnesium & 10400 & & & & UG/L & $06 / 23 / 2009$ & & HCJ-160-09 \\
\hline GWA046012X & USGS-100 & 686 & Manganese & 1 & & U & & UG/L & $06 / 23 / 2009$ & & HCJ-160-09 \\
\hline GWA046012X & USGS-100 & 686 & Mercury & 0.066 & & U & & UG/L & $06 / 23 / 2009$ & & HCJ-160-09 \\
\hline GWA046012X & USGS-100 & 686 & Nickel & 1.05 & & B & & UG/L & $06 / 23 / 2009$ & & HCJ-160-09 \\
\hline GWA046012X & USGS-100 & 686 & Potassium & 3190 & & B & & UG/L & $06 / 23 / 2009$ & & HCJ-160-09 \\
\hline GWA046012X & USGS-100 & 686 & Selenium & 1 & & U & & UG/L & $06 / 23 / 2009$ & & HCJ-160-09 \\
\hline GWA046012X & USGS-100 & 686 & Silver & 0.2 & & $u$ & & UG/L & $06 / 23 / 2009$ & & HCJ-160-09 \\
\hline GWA046012X & USGS-100 & 686 & Sodium & 14300 & & & & UG/L & $06 / 23 / 2009$ & & HCJ-160-09 \\
\hline GWA046012X & USGS-100 & 686 & Strontium & 139 & & & & UG/L & $06 / 23 / 2009$ & & HCJ-160-09 \\
\hline GWA046012X & USGS-100 & 686 & Thallium & 0.3 & & $u$ & & UG/L & $06 / 23 / 2009$ & & HCJ-160-09 \\
\hline GWA046012X & USGS-100 & 686 & Uranium & 1.41 & & B & & UG/L & $06 / 23 / 2009$ & & HCJ-160-09 \\
\hline GWA046012X & USGS-100 & 686 & Vanadium & 3 & & U & & UG/L & $06 / 23 / 2009$ & & HCJ-160-09 \\
\hline GWA046012X & USGS-100 & 686 & Zinc & 189 & & & & UG/L & $06 / 23 / 2009$ & & HCJ-160-09 \\
\hline
\end{tabular}


Table A-2. (continued).

\begin{tabular}{|c|c|c|c|c|c|c|c|c|c|c|c|}
\hline $\begin{array}{c}\text { Field Sample } \\
\text { Number }\end{array}$ & Location & Depth & Compound & $\begin{array}{c}\text { Sample } \\
\text { Result }\end{array}$ & $\begin{array}{c}\text { Sample } \\
\text { Error }\end{array}$ & $\begin{array}{c}\text { Result } \\
\text { Qualifier } \\
\end{array}$ & $\begin{array}{c}\text { Validation } \\
\text { Flag }\end{array}$ & $\begin{array}{c}\text { Sample } \\
\text { Units }\end{array}$ & $\begin{array}{c}\text { Date Sample } \\
\text { Collected }\end{array}$ & MDA & L\&V Report Number \\
\hline GWA04601VL & USGS-100 & 686 & Dichlorodifluoromethane & 1 & & $U$ & & UG/L & $06 / 23 / 2009$ & & HCJ-153-09 \\
\hline GWA04601VL & USGS-100 & 686 & Chloromethane & 1 & & U & & UG/L & $06 / 23 / 2009$ & & HCJ-153-09 \\
\hline GWA04601VL & USGS-100 & 686 & Vinyl Chloride & 1 & & $\mathrm{u}$ & & UG/L & $06 / 23 / 2009$ & & HCJ-153-09 \\
\hline GWA04601VL & USGS-100 & 686 & Bromomethane & 1 & & $\mathrm{U}$ & & UG/L & $06 / 23 / 2009$ & & HCJ-153-09 \\
\hline GWA04601VL & USGS-100 & 686 & Chloroethane & 1 & & U & & UG/L & $06 / 23 / 2009$ & & HCJ-153-09 \\
\hline GWA04601VL & USGS-100 & 686 & Trichlorofluoromethane & 1 & & $\mathrm{U}$ & & UG/L & $06 / 23 / 2009$ & & HCJ-153-09 \\
\hline GWA04601VL & USGS-100 & 686 & Acetone & 5 & & $\mathrm{U}$ & & UG/L & $06 / 23 / 2009$ & & HCJ-153-09 \\
\hline GWA04601VL & USGS-100 & 686 & 1,1-Dichloroethene & 1 & & U & & UG/L & $06 / 23 / 2009$ & & HCJ-153-09 \\
\hline GWA04601VL & USGS-100 & 686 & Methyl acetate & 5 & & U & & UG/L & $06 / 23 / 2009$ & & HCJ-153-09 \\
\hline GWA04601VL & USGS-100 & 686 & Methylene Chloride & 5 & & $\mathrm{U}$ & & UG/L & $06 / 23 / 2009$ & & HCJ-153-09 \\
\hline GWA04601VL & USGS-100 & 686 & Carbon disulfide & 5 & & $\mathrm{U}$ & & UG/L & $06 / 23 / 2009$ & & HCJ-153-09 \\
\hline GWA04601VL & USGS-100 & 686 & Tert-butyl methyl ether & 1 & & $U$ & & UG/L & $06 / 23 / 2009$ & & HCJ-153-09 \\
\hline GWA04601VL & USGS-100 & 686 & trans-1,2-Dichloroethylene & 1 & & $\mathrm{U}$ & & UG/L & $06 / 23 / 2009$ & & HCJ-153-09 \\
\hline GWA04601VL & USGS-100 & 686 & 1,1-Dichloroethane & 1 & & $\mathrm{u}$ & & UG/L & $06 / 23 / 2009$ & & HCJ-153-09 \\
\hline GWA04601VL & USGS-100 & 686 & 2-Butanone & 5 & & U & & UG/L & $06 / 23 / 2009$ & & HCJ-153-09 \\
\hline GWA04601VL & USGS-100 & 686 & cis-1,2-Dichloroethylene & 1 & & U & & UG/L & $06 / 23 / 2009$ & & HCJ-153-09 \\
\hline GWA04601VL & USGS-100 & 686 & Chloroform & 1 & & $\mathrm{U}$ & & UG/L & $06 / 23 / 2009$ & & HCJ-153-09 \\
\hline GWA04601VL & USGS-100 & 686 & 1,1,1-Trichloroethane & 1 & & $\mathrm{U}$ & & UG/L & $06 / 23 / 2009$ & & HCJ-153-09 \\
\hline GWA04601VL & USGS-100 & 686 & Cyclohexane & 1 & & U & & UG/L & $06 / 23 / 2009$ & & HCJ-153-09 \\
\hline GWA04601VL & USGS-100 & 686 & Carbon tetrachloride & 1 & & $u$ & & UG/L & $06 / 23 / 2009$ & & HCJ-153-09 \\
\hline GWA04601VL & USGS-100 & 686 & 1,2-Dichloroethane & 1 & & $\mathrm{U}$ & & UG/L & $06 / 23 / 2009$ & & HCJ-153-09 \\
\hline GWA04601VL & USGS-100 & 686 & Benzene & 1 & & U & & UG/L & $06 / 23 / 2009$ & & HCJ-153-09 \\
\hline GWA04601VL & USGS-100 & 686 & Trichloroethylene & 1 & & U & & UG/L & $06 / 23 / 2009$ & & HCJ-153-09 \\
\hline GWA04601VL & USGS-100 & 686 & 1,2-Dichloropropane & 1 & & U & & UG/L & $06 / 23 / 2009$ & & HCJ-153-09 \\
\hline GWA04601VL & USGS-100 & 686 & Methyl cyclohexane & 1 & & $\mathrm{U}$ & & UG/L & $06 / 23 / 2009$ & & HCJ-153-09 \\
\hline GWA04601VL & USGS-100 & 686 & Bromodichloromethane & 1 & & U & & UG/L & $06 / 23 / 2009$ & & HCJ-153-09 \\
\hline GWA04601VL & USGS-100 & 686 & 4-Methyl-2-pentanone & 5 & & $\mathrm{U}$ & & UG/L & $06 / 23 / 2009$ & & HCJ-153-09 \\
\hline GWA04601VL & USGS-100 & 686 & cis-1,3-Dichloropropylene & 1 & & $\mathrm{u}$ & & UG/L & $06 / 23 / 2009$ & & HCJ-153-09 \\
\hline GWA04601VL & USGS-100 & 686 & Toluene & 1 & & U & & UG/L & $06 / 23 / 2009$ & & HCJ-153-09 \\
\hline GWA04601VL & USGS-100 & 686 & trans-1,3-Dichloropropylene & 1 & & U & & UG/L & $06 / 23 / 2009$ & & HCJ-153-09 \\
\hline GWA04601VL & USGS-100 & 686 & 1,1,2-Trichloroethane & 1 & & $u$ & & UG/L & $06 / 23 / 2009$ & & HCJ-153-09 \\
\hline GWA04601VL & USGS-100 & 686 & 2-Hexanone & 5 & & $\mathrm{u}$ & & UG/L & $06 / 23 / 2009$ & & HCJ-153-09 \\
\hline GWA04601VL & USGS-100 & 686 & Tetrachloroethylene & 1 & & $\mathrm{U}$ & & UG/L & $06 / 23 / 2009$ & & HCJ-153-09 \\
\hline GWA04601VL & USGS-100 & 686 & Dibromochloromethane & 1 & & $U$ & & UG/L & $06 / 23 / 2009$ & & HCJ-153-09 \\
\hline GWA04601VL & USGS-100 & 686 & 1,2-Dibromoethane & 1 & & $\mathrm{u}$ & & UG/L & $06 / 23 / 2009$ & & HCJ-153-09 \\
\hline GWA04601VL & USGS-100 & 686 & Chlorobenzene & 1 & & $\mathrm{U}$ & & UG/L & $06 / 23 / 2009$ & & HCJ-153-09 \\
\hline GWA04601VL & USGS-100 & 686 & Ethylbenzene & 1 & & U & & UG/L & $06 / 23 / 2009$ & & HCJ-153-09 \\
\hline GWA04601VL & USGS-100 & 686 & Styrene & 1 & & $U$ & & UG/L & $06 / 23 / 2009$ & & HCJ-153-09 \\
\hline GWA04601VL & USGS-100 & 686 & Bromoform & 1 & & $\mathrm{U}$ & & UG/L & $06 / 23 / 2009$ & & HCJ-153-09 \\
\hline GWA04601VL & USGS-100 & 686 & 1,1,2,2-Tetrachloroethane & 1 & & $\mathrm{U}$ & & UG/L & $06 / 23 / 2009$ & & HCJ-153-09 \\
\hline GWA04601VL & USGS-100 & 686 & Isopropylbenzene & 1 & & $\mathrm{U}$ & & UG/L & $06 / 23 / 2009$ & & HCJ-153-09 \\
\hline GWA04601VL & USGS-100 & 686 & 1,3-Dichlorobenzene & 1 & & $\mathrm{U}$ & & UG/L & $06 / 23 / 2009$ & & HCJ-153-09 \\
\hline GWA04601VL & USGS-100 & 686 & 1,4-Dichlorobenzene & 1 & & $u$ & & UG/L & $06 / 23 / 2009$ & & HCJ-153-09 \\
\hline GWA04601VL & USGS-100 & 686 & 1,2-Dibromo-3-chloropropane & 1 & & $\mathrm{U}$ & & UG/L & $06 / 23 / 2009$ & & HCJ-153-09 \\
\hline
\end{tabular}


Table A-2 (continued).

\begin{tabular}{|c|c|c|c|c|c|c|c|c|c|c|c|}
\hline $\begin{array}{c}\text { Field Sample } \\
\text { Number }\end{array}$ & Location & Depth & Compound & $\begin{array}{c}\text { Sample } \\
\text { Result }\end{array}$ & $\begin{array}{l}\text { Sample } \\
\text { Error }\end{array}$ & $\begin{array}{c}\text { Result } \\
\text { Qualifier }\end{array}$ & $\begin{array}{c}\text { Validation } \\
\text { Flag }\end{array}$ & $\begin{array}{c}\text { Sample } \\
\text { Units }\end{array}$ & $\begin{array}{c}\text { Date Sample } \\
\text { Collected }\end{array}$ & MDA & L\&V Report Number \\
\hline GWA04601VL & USGS-100 & 686 & Trichlorotrifluoroethane & 5 & & $U$ & & UG/L & $06 / 23 / 2009$ & & HCJ-153-09 \\
\hline GWA04601VL & USGS-100 & 686 & Xylene (Total) & 1 & & U & & UG/L & $06 / 23 / 2009$ & & HCJ-153-09 \\
\hline GWA04601VL & USGS-100 & 686 & 1,2,4-Trichlorobenzene & 1 & & $\mathrm{U}$ & & UG/L & $06 / 23 / 2009$ & & HCJ-153-09 \\
\hline GWA04601VL & USGS-100 & 686 & 1,2-Dichlorobenzene & 1 & & $\mathrm{U}$ & & UG/L & $06 / 23 / 2009$ & & HCJ-153-09 \\
\hline GWA04601R8 & USGS-100 & 686 & Tritium & $2.58 \mathrm{E}+01$ & $1.09 \mathrm{E}+02$ & & U & $\mathrm{PCI} / \mathrm{L}$ & $06 / 23 / 2009$ & $3.85 \mathrm{E}+02$ & BAM-119-09 \\
\hline GWA04601RH & USGS-100 & 686 & Gross Alpha & $2.04 \mathrm{E}+00$ & 7.04E-01 & & $\mathrm{J}$ & $\mathrm{PCI} / \mathrm{L}$ & $06 / 23 / 2009$ & $1.44 \mathrm{E}+00$ & BAM-120-09 \\
\hline GWA04601RH & USGS-100 & 686 & Gross Beta & $2.57 \mathrm{E}+00$ & $6.48 \mathrm{E}-01$ & & & $\mathrm{PCl} / \mathrm{L}$ & $06 / 23 / 2009$ & $1.51 \mathrm{E}+00$ & BAM-120-09 \\
\hline GWA04601RH & USGS-100 & 686 & Strontium-90 & $5.90 \mathrm{E}-02$ & 1.15E-01 & & U & $\mathrm{PCl} / \mathrm{L}$ & $06 / 23 / 2009$ & 4.00E-01 & BAM-120-09 \\
\hline GWA04601RH & USGS-100 & 686 & Technetium-99 & $-2.01 \mathrm{E}+00$ & $1.63 E+00$ & & U & $\mathrm{PCI} / \mathrm{L}$ & $06 / 23 / 2009$ & $5.87 \mathrm{E}+00$ & BAM-120-09 \\
\hline GWA04601UX & USGS-100 & 686 & lodine-129 & $-2.10 \mathrm{E}-02$ & 1.93E-02 & & U & $\mathrm{PCl} / \mathrm{L}$ & $06 / 23 / 2009$ & 5.66E-02 & BAM-118-09 \\
\hline GWA033012X & USGS-101 & 771 & Aluminum & 15 & & $\mathrm{U}$ & & UG/L & $06 / 03 / 2009$ & & HCJ-161-09 \\
\hline GWA033012X & USGS-101 & 771 & Antimony & 1 & & $U$ & & UG/L & $06 / 03 / 2009$ & & HCJ-161-09 \\
\hline GWA033012X & USGS-101 & 771 & Arsenic & 1.6 & & U & & UG/L & 06/03/2009 & & HCJ-161-09 \\
\hline GWA033012X & USGS-101 & 771 & Barium & 17.7 & & B & & UG/L & $06 / 03 / 2009$ & & HCJ-161-09 \\
\hline GWA033012X & USGS-101 & 771 & Beryllium & 0.1 & & U & & UG/L & $06 / 03 / 2009$ & & HCJ-161-09 \\
\hline GWA033012X & USGS-101 & 771 & Cadmium & 0.11 & & U & & UG/L & 06/03/2009 & & HCJ-161-09 \\
\hline GWA033012X & USGS-101 & 771 & Calcium & 26800 & & & & UG/L & 06/03/2009 & & HCJ-161-09 \\
\hline GWA033012X & USGS-101 & 771 & Chromium & 2 & & U & & UG/L & $06 / 03 / 2009$ & & HCJ-161-09 \\
\hline GWA033012X & USGS-101 & 771 & Cobalt & 3.55 & & B & & UG/L & $06 / 03 / 2009$ & & HCJ-161-09 \\
\hline GWA033012X & USGS-101 & 771 & Copper & 0.6 & & B & & UG/L & 06/03/2009 & & HCJ-161-09 \\
\hline GWA033012X & USGS-101 & 771 & Iron & 140 & & & & UG/L & 06/03/2009 & & HCJ-161-09 \\
\hline GWA033012X & USGS-101 & 771 & Lead & 0.5 & & U & & UG/L & 06/03/2009 & & HCJ-161-09 \\
\hline GWA033012X & USGS-101 & 771 & Magnesium & 8930 & & & & UG/L & $06 / 03 / 2009$ & & HCJ-161-09 \\
\hline GWA033012X & USGS-101 & 771 & Manganese & 6.82 & & B & & UG/L & 06/03/2009 & & HCJ-161-09 \\
\hline GWA033012X & USGS-101 & 771 & Mercury & 0.067 & & U & & UG/L & $06 / 03 / 2009$ & & HCJ-161-09 \\
\hline GWA033012X & USGS-101 & 771 & Nickel & 1.1 & & B & & UG/L & $06 / 03 / 2009$ & & HCJ-161-09 \\
\hline GWA033012X & USGS-101 & 771 & Potassium & 2600 & & B & & UG/L & 06/03/2009 & & HCJ-161-09 \\
\hline GWA033012X & USGS-101 & 771 & Selenium & 1 & & U & & UG/L & 06/03/2009 & & HCJ-161-09 \\
\hline GWA033012X & USGS-101 & 771 & Silver & 0.2 & & U & & UG/L & $06 / 03 / 2009$ & & HCJ-161-09 \\
\hline GWA033012X & USGS-101 & 771 & Sodium & 14100 & & & & UG/L & $06 / 03 / 2009$ & & HCJ-161-09 \\
\hline GWA033012X & USGS-101 & 771 & Strontium & 91.9 & & & & UG/L & 06/03/2009 & & HCJ-161-09 \\
\hline GWA033012X & USGS-101 & 771 & Thallium & 0.3 & & U & & UG/L & $06 / 03 / 2009$ & & HCJ-161-09 \\
\hline GWA033012X & USGS-101 & 771 & Uranium & 1.79 & & B & & UG/L & $06 / 03 / 2009$ & & HCJ-161-09 \\
\hline GWA033012X & USGS-101 & 771 & Vanadium & 3.05 & & B & & UG/L & $06 / 03 / 2009$ & & HCJ-161-09 \\
\hline GWA033012X & USGS-101 & 771 & Zinc & 5.44 & & B & & UG/L & 06/03/2009 & & HCJ-161-09 \\
\hline GWA03301A1 & USGS-101 & 771 & Alkalinity, Total as $\mathrm{CaCO} 3$ & 116 & & & & $M G / L$ & $06 / 03 / 2009$ & & HCJ-157-09 \\
\hline GWA03301AN & USGS-101 & 771 & Bromide & 0 & & U & & $M G / L$ & $06 / 03 / 2009$ & & HCJ-159-09 \\
\hline GWA03301AN & USGS-101 & 771 & Chloride & 9.11 & & & & $M G / L$ & $06 / 03 / 2009$ & & HCJ-159-09 \\
\hline GWA03301AN & USGS-101 & 771 & Fluoride & 0.896 & & & & $M G / L$ & $06 / 03 / 2009$ & & HCJ-159-09 \\
\hline GWA03301AN & USGS-101 & 771 & Sulfate & 10.3 & & & & $M G / L$ & $06 / 03 / 2009$ & & HCJ-159-09 \\
\hline GWA03301N2 & USGS-101 & 771 & Nitrogen, Nitrate/Nitrite & 1.18 & & & & $M G / L$ & $06 / 03 / 2009$ & & HCJ-155-09 \\
\hline GWA03301VL & USGS-101 & 771 & Dichlorodifluoromethane & 1 & & $\mathrm{u}$ & & UG/L & $06 / 03 / 2009$ & & HCJ-148-09 \\
\hline GWA03301VL & USGS-101 & 771 & Chloromethane & 1 & & $U$ & & UG/L & $06 / 03 / 2009$ & & HCJ-148-09 \\
\hline GWA03301VL & USGS-101 & 771 & Vinyl Chloride & 1 & & U & & UG/L & $06 / 03 / 2009$ & & HCJ-148-09 \\
\hline
\end{tabular}


Table A-2. (continued).

\begin{tabular}{|c|c|c|c|c|c|c|c|c|c|c|c|}
\hline $\begin{array}{c}\text { Field Sample } \\
\text { Number }\end{array}$ & Location & Depth & Compound & $\begin{array}{c}\text { Sample } \\
\text { Result }\end{array}$ & $\begin{array}{c}\text { Sample } \\
\text { Error }\end{array}$ & $\begin{array}{c}\text { Result } \\
\text { Qualifier } \\
\end{array}$ & $\begin{array}{c}\text { Validation } \\
\text { Flag }\end{array}$ & $\begin{array}{c}\text { Sample } \\
\text { Units }\end{array}$ & $\begin{array}{c}\text { Date Sample } \\
\text { Collected }\end{array}$ & MDA & L\&V Report Number \\
\hline GWA03301VL & USGS-101 & 771 & Bromomethane & 1 & & $U$ & & UG/L & $06 / 03 / 2009$ & & HCJ-148-09 \\
\hline GWA03301VL & USGS-101 & 771 & Chloroethane & 1 & & U & & UG/L & $06 / 03 / 2009$ & & HCJ-148-09 \\
\hline GWA03301VL & USGS-101 & 771 & Trichlorofluoromethane & 1 & & $\mathrm{U}$ & & UG/L & 06/03/2009 & & HCJ-148-09 \\
\hline GWA03301VL & USGS-101 & 771 & Acetone & 5 & & $\mathrm{U}$ & & UG/L & $06 / 03 / 2009$ & & HCJ-148-09 \\
\hline GWA03301VL & USGS-101 & 771 & 1,1-Dichloroethene & 1 & & U & & UG/L & $06 / 03 / 2009$ & & HCJ-148-09 \\
\hline GWA03301VL & USGS-101 & 771 & Methyl acetate & 5 & & $\mathrm{U}$ & & UG/L & 06/03/2009 & & HCJ-148-09 \\
\hline GWA03301VL & USGS-101 & 771 & Methylene Chloride & 5 & & $\mathrm{U}$ & & UG/L & $06 / 03 / 2009$ & & HCJ-148-09 \\
\hline GWA03301VL & USGS-101 & 771 & Carbon disulfide & 5 & & U & & UG/L & $06 / 03 / 2009$ & & HCJ-148-09 \\
\hline GWA03301VL & USGS-101 & 771 & Tert-butyl methyl ether & 1 & & U & & UG/L & $06 / 03 / 2009$ & & HCJ-148-09 \\
\hline GWA03301VL & USGS-101 & 771 & trans-1,2-Dichloroethylene & 1 & & $\mathrm{U}$ & & UG/L & 06/03/2009 & & HCJ-148-09 \\
\hline GWA03301VL & USGS-101 & 771 & 1,1-Dichloroethane & 1 & & $\mathrm{U}$ & & UG/L & $06 / 03 / 2009$ & & HCJ-148-09 \\
\hline GWA03301VL & USGS-101 & 771 & 2-Butanone & 5 & & $U$ & & UG/L & $06 / 03 / 2009$ & & HCJ-148-09 \\
\hline GWA03301VL & USGS-101 & 771 & cis-1,2-Dichloroethylene & 1 & & U & & UG/L & $06 / 03 / 2009$ & & HCJ-148-09 \\
\hline GWA03301VL & USGS-101 & 771 & Chloroform & 1 & & $\mathrm{U}$ & & UG/L & $06 / 03 / 2009$ & & HCJ-148-09 \\
\hline GWA03301VL & USGS-101 & 771 & 1,1,1-Trichloroethane & 1 & & U & & UG/L & $06 / 03 / 2009$ & & HCJ-148-09 \\
\hline GWA03301VL & USGS-101 & 771 & Cyclohexane & 1 & & U & & UG/L & $06 / 03 / 2009$ & & HCJ-148-09 \\
\hline GWA03301VL & USGS-101 & 771 & Carbon tetrachloride & 1 & & $u$ & & UG/L & $06 / 03 / 2009$ & & HCJ-148-09 \\
\hline GWA03301VL & USGS-101 & 771 & 1,2-Dichloroethane & 1 & & $\mathrm{U}$ & & UG/L & $06 / 03 / 2009$ & & HCJ-148-09 \\
\hline GWA03301VL & USGS-101 & 771 & Benzene & 1 & & U & & UG/L & $06 / 03 / 2009$ & & HCJ-148-09 \\
\hline GWA03301VL & USGS-101 & 771 & Trichloroethylene & 1 & & $u$ & & UG/L & $06 / 03 / 2009$ & & HCJ-148-09 \\
\hline GWA03301VL & USGS-101 & 771 & 1,2-Dichloropropane & 1 & & $\mathrm{U}$ & & UG/L & 06/03/2009 & & HCJ-148-09 \\
\hline GWA03301VL & USGS-101 & 771 & Methyl cyclohexane & 1 & & U & & UG/L & $06 / 03 / 2009$ & & HCJ-148-09 \\
\hline GWA03301VL & USGS-101 & 771 & Bromodichloromethane & 1 & & U & & UG/L & $06 / 03 / 2009$ & & HCJ-148-09 \\
\hline GWA03301VL & USGS-101 & 771 & 4-Methyl-2-pentanone & 5 & & U & & UG/L & $06 / 03 / 2009$ & & HCJ-148-09 \\
\hline GWA03301VL & USGS-101 & 771 & cis-1,3-Dichloropropylene & 1 & & $\mathrm{U}$ & & UG/L & $06 / 03 / 2009$ & & HCJ-148-09 \\
\hline GWA03301VL & USGS-101 & 771 & Toluene & 1 & & U & & UG/L & $06 / 03 / 2009$ & & HCJ-148-09 \\
\hline GWA03301VL & USGS-101 & 771 & trans-1,3-Dichloropropylene & 1 & & $\mathrm{U}$ & & UG/L & $06 / 03 / 2009$ & & HCJ-148-09 \\
\hline GWA03301VL & USGS-101 & 771 & 1,1,2-Trichloroethane & 1 & & $\mathrm{u}$ & & UG/L & $06 / 03 / 2009$ & & HCJ-148-09 \\
\hline GWA03301VL & USGS-101 & 771 & 2-Hexanone & 5 & & U & & UG/L & $06 / 03 / 2009$ & & HCJ-148-09 \\
\hline GWA03301VL & USGS-101 & 771 & Tetrachloroethylene & 1 & & U & & UG/L & $06 / 03 / 2009$ & & HCJ-148-09 \\
\hline GWA03301VL & USGS-101 & 771 & Dibromochloromethane & 1 & & $u$ & & UG/L & $06 / 03 / 2009$ & & HCJ-148-09 \\
\hline GWA03301VL & USGS-101 & 771 & 1,2-Dibromoethane & 1 & & $\mathrm{u}$ & & UG/L & $06 / 03 / 2009$ & & HCJ-148-09 \\
\hline GWA03301VL & USGS-101 & 771 & Chlorobenzene & 1 & & $\mathrm{U}$ & & UG/L & $06 / 03 / 2009$ & & HCJ-148-09 \\
\hline GWA03301VL & USGS-101 & 771 & Ethylbenzene & 1 & & $U$ & & UG/L & $06 / 03 / 2009$ & & HCJ-148-09 \\
\hline GWA03301VL & USGS-101 & 771 & Styrene & 1 & & $\mathrm{u}$ & & UG/L & $06 / 03 / 2009$ & & HCJ-148-09 \\
\hline GWA03301VL & USGS-101 & 771 & Bromoform & 1 & & U & & UG/L & $06 / 03 / 2009$ & & HCJ-148-09 \\
\hline GWA03301VL & USGS-101 & 771 & 1,1,2,2-Tetrachloroethane & 1 & & $\mathrm{U}$ & & UG/L & $06 / 03 / 2009$ & & HCJ-148-09 \\
\hline GWA03301VL & USGS-101 & 771 & Isopropylbenzene & 1 & & $u$ & & UG/L & $06 / 03 / 2009$ & & HCJ-148-09 \\
\hline GWA03301VL & USGS-101 & 771 & 1,3-Dichlorobenzene & 1 & & $\mathrm{U}$ & & UG/L & $06 / 03 / 2009$ & & HCJ-148-09 \\
\hline GWA03301VL & USGS-101 & 771 & 1,4-Dichlorobenzene & 1 & & $\mathrm{U}$ & & UG/L & $06 / 03 / 2009$ & & HCJ-148-09 \\
\hline GWA03301VL & USGS-101 & 771 & 1,2-Dibromo-3-chloropropane & 1 & & $\mathrm{U}$ & & UG/L & $06 / 03 / 2009$ & & HCJ-148-09 \\
\hline GWA03301VL & USGS-101 & 771 & Trichlorotrifluoroethane & 5 & & $\mathrm{U}$ & & UG/L & $06 / 03 / 2009$ & & HCJ-148-09 \\
\hline GWA03301VL & USGS-101 & 771 & Xylene (Total) & 1 & & $u$ & & UG/L & $06 / 03 / 2009$ & & HCJ-148-09 \\
\hline GWA03301VL & USGS-101 & 771 & 1,2,4-Trichlorobenzene & 1 & & $\mathrm{U}$ & & UG/L & 06/03/2009 & & HCJ-148-09 \\
\hline
\end{tabular}


Table A-2. (continued).

\begin{tabular}{|c|c|c|c|c|c|c|c|c|c|c|c|}
\hline $\begin{array}{c}\text { Field Sample } \\
\text { Number }\end{array}$ & Location & Depth & Compound & $\begin{array}{l}\text { Sample } \\
\text { Result }\end{array}$ & $\begin{array}{c}\text { Sample } \\
\text { Error }\end{array}$ & $\begin{array}{c}\text { Result } \\
\text { Qualifier }\end{array}$ & $\begin{array}{c}\text { Validation } \\
\text { Flag }\end{array}$ & $\begin{array}{c}\text { Sample } \\
\text { Units }\end{array}$ & $\begin{array}{c}\text { Date Sample } \\
\text { Collected }\end{array}$ & MDA & L\&V Report Number \\
\hline GWA03301VL & USGS-101 & 771 & 1,2-Dichlorobenzene & 1 & & $\mathrm{U}$ & & UG/L & $06 / 03 / 2009$ & & HCJ-148-09 \\
\hline GWA03301R8 & USGS-101 & 771 & Tritium & $-6.45 E+01$ & $8.57 \mathrm{E}+01$ & & $u$ & $\mathrm{PCl} / \mathrm{L}$ & $06 / 03 / 2009$ & $3.05 \mathrm{E}+02$ & BAM-119-09 \\
\hline GWA03301RH & USGS-101 & 771 & Gross Alpha & 7.31E-01 & 4.18E-01 & & $u$ & $\mathrm{PCl} / \mathrm{L}$ & $06 / 03 / 2009$ & $1.17 \mathrm{E}+00$ & BAM-120-09 \\
\hline GWA03301RH & USGS-101 & 771 & Gross Beta & $6.25 \mathrm{E}+00$ & $1.17 \mathrm{E}+00$ & & & $\mathrm{PCl} / \mathrm{L}$ & $06 / 03 / 2009$ & $2.75 \mathrm{E}+00$ & BAM-120-09 \\
\hline GWA03301RH & USGS-101 & 771 & Strontium-90 & $-4.67 \mathrm{E}-02$ & $9.46 \mathrm{E}-02$ & & U & $\mathrm{PCl} / \mathrm{L}$ & $06 / 03 / 2009$ & 3.51E-01 & BAM-120-09 \\
\hline GWA03301RH & USGS-101 & 771 & Technetium-99 & 3.83E-01 & $1.66 \mathrm{E}+00$ & & U & $\mathrm{PCl} / \mathrm{L}$ & $06 / 03 / 2009$ & $5.74 \mathrm{E}+00$ & BAM-120-09 \\
\hline GWA03301UX & USGS-101 & 771 & lodine-129 & $5.27 \mathrm{E}-03$ & $2.46 \mathrm{E}-02$ & & $u$ & $\mathrm{PCl} / \mathrm{L}$ & 06/03/2009 & 8.33E-02 & BAM-118-09 \\
\hline GWA03401A1 & USGS-103 & 681.9 & Alkalinity, Total as $\mathrm{CaCO} 3$ & 104 & & & & MG/L & 07/01/2009 & & HCJ-168-09 \\
\hline GWA03501A1 & USGS-103 & 805.2 & Alkalinity, Total as $\mathrm{CaCO} 3$ & 126 & & & & $M G / L$ & $07 / 01 / 2009$ & & HCJ-168-09 \\
\hline GWA03601A1 & USGS-103 & 913.8 & Alkalinity, Total as $\mathrm{CaCO} 3$ & 123 & & & & $M G / L$ & $07 / 02 / 2009$ & & HCJ-168-09 \\
\hline GWA03701A1 & USGS-103 & 999.4 & Alkalinity, Total as $\mathrm{CaCO} 3$ & 131 & & & & $M G / L$ & $07 / 07 / 2009$ & & HCJ-168-09 \\
\hline GWA03801A1 & USGS-103 & 1095.1 & Alkalinity, Total as $\mathrm{CaCO} 3$ & 145 & & & & $M G / L$ & 07/08/2009 & & HCJ-168-09 \\
\hline GWA03901A1 & USGS-103 & 1220.2 & Alkalinity, Total as $\mathrm{CaCO} 3$ & 138 & & & & $M G / L$ & $07 / 09 / 2009$ & & HCJ-168-09 \\
\hline GWA03401AN & USGS-103 & 681.9 & Bromide & 0 & & $u$ & & MG/L & 07/01/2009 & & HCJ-170-09 \\
\hline GWA03401AN & USGS-103 & 681.9 & Chloride & 19.2 & & & $\mathrm{~J}$ & $M G / L$ & 07/01/2009 & & HCJ-170-09 \\
\hline GWA03401AN & USGS-103 & 681.9 & Fluoride & 0.386 & & $\mathrm{~J}$ & & $M G / L$ & $07 / 01 / 2009$ & & HCJ-170-09 \\
\hline GWA03401AN & USGS-103 & 681.9 & Sulfate & 18 & & & & $M G / L$ & $07 / 01 / 2009$ & & HCJ-170-09 \\
\hline GWA03501AN & USGS-103 & 805.2 & Bromide & 0 & & $u$ & & MG/L & 07/01/2009 & & HCJ-170-09 \\
\hline GWA03501AN & USGS-103 & 805.2 & Chloride & 13.7 & & & $\mathrm{~J}$ & MG/L & $07 / 01 / 2009$ & & HCJ-170-09 \\
\hline GWA03501AN & USGS-103 & 805.2 & Fluoride & 0.296 & & $\mathrm{~J}$ & & $M G / L$ & $07 / 01 / 2009$ & & HCJ-170-09 \\
\hline GWA03501AN & USGS-103 & 805.2 & Sulfate & 21.8 & & & & MG/L & $07 / 01 / 2009$ & & HCJ-170-09 \\
\hline GWA03601AN & USGS-103 & 913.8 & Bromide & 0 & & U & & $M G / L$ & $07 / 02 / 2009$ & & HCJ-170-09 \\
\hline GWA03601AN & USGS-103 & 913.8 & Chloride & 9.38 & & & $\mathrm{~J}$ & $M G / L$ & $07 / 02 / 2009$ & & HCJ-170-09 \\
\hline GWA03601AN & USGS-103 & 913.8 & Fluoride & 0.196 & & $\mathrm{~J}$ & & MG/L & $07 / 02 / 2009$ & & HCJ-170-09 \\
\hline GWA03601AN & USGS-103 & 913.8 & Sulfate & 18 & & & & MG/L & $07 / 02 / 2009$ & & HCJ-170-09 \\
\hline GWA03701AN & USGS-103 & 999.4 & Bromide & 0 & & U & & $M G / L$ & $07 / 07 / 2009$ & & HCJ-170-09 \\
\hline GWA03701AN & USGS-103 & 999.4 & Chloride & 11.7 & & & $\mathrm{~J}$ & $M G / L$ & $07 / 07 / 2009$ & & HCJ-170-09 \\
\hline GWA03701AN & USGS-103 & 999.4 & Fluoride & 0.195 & & $\mathrm{~J}$ & & MG/L & $07 / 07 / 2009$ & & HCJ-170-09 \\
\hline GWA03701AN & USGS-103 & 999.4 & Sulfate & 19.9 & & & & MG/L & $07 / 07 / 2009$ & & HCJ-170-09 \\
\hline GWA03801AN & USGS-103 & 1095.1 & Bromide & 0 & & U & & $M G / L$ & 07/09/2009 & & HCJ-170-09 \\
\hline GWA03801AN & USGS-103 & 1095.1 & Chloride & 13.6 & & & $\mathrm{~J}$ & $M G / L$ & $07 / 09 / 2009$ & & HCJ-170-09 \\
\hline GWA03801AN & USGS-103 & 1095.1 & Fluoride & 0.178 & & $\mathrm{~J}$ & & MG/L & $07 / 09 / 2009$ & & HCJ-170-09 \\
\hline GWA03801AN & USGS-103 & 1095.1 & Sulfate & 21.3 & & & & $M G / L$ & 07/09/2009 & & HCJ-170-09 \\
\hline GWA03901AN & USGS-103 & 1220.2 & Bromide & 0 & & $U$ & & $M G / L$ & $07 / 09 / 2009$ & & HCJ-170-09 \\
\hline GWA03901AN & USGS-103 & 1220.2 & Chloride & 13.6 & & & $\mathrm{~J}$ & MG/L & $07 / 09 / 2009$ & & HCJ-170-09 \\
\hline GWA03901AN & USGS-103 & 1220.2 & Fluoride & 0.178 & & $\mathrm{~J}$ & & MG/L & $07 / 09 / 2009$ & & HCJ-170-09 \\
\hline GWA03901AN & USGS-103 & 1220.2 & Sulfate & 21.3 & & & & $M G / L$ & 07/09/2009 & & HCJ-170-09 \\
\hline GWA04001AN & USGS-103 & 1269.4 & Bromide & 0 & & U & & $M G / L$ & $07 / 13 / 2009$ & & HCJ-170-09 \\
\hline GWA04001AN & USGS-103 & 1269.4 & Chloride & 13.5 & & & $\mathrm{~J}$ & MG/L & $07 / 13 / 2009$ & & HCJ-170-09 \\
\hline GWA04001AN & USGS-103 & 1269.4 & Fluoride & 0.183 & & $\mathrm{~J}$ & & $M G / L$ & $07 / 13 / 2009$ & & HCJ-170-09 \\
\hline GWA04001AN & USGS-103 & 1269.4 & Sulfate & 21.2 & & & & $M G / L$ & $07 / 13 / 2009$ & & HCJ-170-09 \\
\hline GWA03401N2 & USGS-103 & 681.9 & Nitrogen, Nitrate/Nitrite & 0.232 & & & & MG/L & $07 / 01 / 2009$ & & HCJ-171-09 \\
\hline GWA03501N2 & USGS-103 & 805.2 & Nitrogen, Nitrate/Nitrite & 0.651 & & & & MG/L & $07 / 01 / 2009$ & & HCJ-171-09 \\
\hline GWA03601N2 & USGS-103 & 913.8 & Nitrogen, Nitrate/Nitrite & 0.505 & & & & $M G / L$ & $07 / 02 / 2009$ & & HCJ-171-09 \\
\hline
\end{tabular}


Table A-2. (continued).

\begin{tabular}{|c|c|c|c|c|c|c|c|c|c|c|c|}
\hline $\begin{array}{c}\text { Field Sample } \\
\text { Number }\end{array}$ & Location & Depth & Compound & $\begin{array}{c}\text { Sample } \\
\text { Result }\end{array}$ & $\begin{array}{c}\text { Sample } \\
\text { Error }\end{array}$ & $\begin{array}{c}\text { Result } \\
\text { Qualifier }\end{array}$ & $\begin{array}{c}\text { Validation } \\
\text { Flag }\end{array}$ & $\begin{array}{c}\text { Sample } \\
\text { Units }\end{array}$ & $\begin{array}{c}\text { Date Sample } \\
\text { Collected }\end{array}$ & MDA & L\&V Report Number \\
\hline GWA03701N2 & USGS-103 & 999.4 & Nitrogen, Nitrate/Nitrite & 0.666 & & & & MG/L & $07 / 07 / 2009$ & & HCJ-171-09 \\
\hline GWA03801N2 & USGS-103 & 1095.1 & Nitrogen, Nitrate/Nitrite & 0.762 & & & & MG/L & 07/08/2009 & & HCJ-171-09 \\
\hline GWA03901N2 & USGS-103 & 1220.2 & Nitrogen, Nitrate/Nitrite & 0.794 & & & & MG/L & 07/09/2009 & & HCJ-171-09 \\
\hline GWA04001N2 & USGS-103 & 1269.4 & Nitrogen, Nitrate/Nitrite & 0.82 & & & & MG/L & 07/13/2009 & & HCJ-171-09 \\
\hline GWA04001A1 & USGS-103 & 1269.4 & Alkalinity, Total as $\mathrm{CaCO} 3$ & 139 & & & & MG/L & 07/13/2009 & & HCJ-169-09 \\
\hline GWA034012X & USGS-103 & 681.9 & Aluminum & 15 & & $\mathrm{U}$ & & UG/L & 07/01/2009 & & HCJ-172-09 \\
\hline GWA034012X & USGS-103 & 681.9 & Antimony & 1 & & U & & UG/L & 07/01/2009 & & HCJ-172-09 \\
\hline GWA034012X & USGS-103 & 681.9 & Arsenic & 1.6 & & U & & UG/L & 07/01/2009 & & HCJ-172-09 \\
\hline GWA034012X & USGS-103 & 681.9 & Barium & 40.8 & & B & & UG/L & 07/01/2009 & & HCJ-172-09 \\
\hline GWA034012X & USGS-103 & 681.9 & Beryllium & 0.1 & & U & & UG/L & 07/01/2009 & & HCJ-172-09 \\
\hline GWA034012X & USGS-103 & 681.9 & Cadmium & 0.11 & & U & & UG/L & 07/01/2009 & & HCJ-172-09 \\
\hline GWA034012X & USGS-103 & 681.9 & Calcium & 22100 & & & & UG/L & 07/01/2009 & & HCJ-172-09 \\
\hline GWA034012X & USGS-103 & 681.9 & Chromium & 2 & & $U$ & & UG/L & 07/01/2009 & & HCJ-172-09 \\
\hline GWA034012X & USGS-103 & 681.9 & Cobalt & 0.106 & & B & & UG/L & 07/01/2009 & & HCJ-172-09 \\
\hline GWA034012X & USGS-103 & 681.9 & Copper & 0.742 & & B & & UG/L & 07/01/2009 & & HCJ-172-09 \\
\hline GWA034012X & USGS-103 & 681.9 & Iron & 163 & & & & UG/L & 07/01/2009 & & HCJ-172-09 \\
\hline GWA034012X & USGS-103 & 681.9 & Lead & 0.5 & & $\mathrm{U}$ & & UG/L & 07/01/2009 & & HCJ-172-09 \\
\hline GWA034012X & USGS-103 & 681.9 & Magnesium & 15000 & & & & UG/L & 07/01/2009 & & HCJ-172-09 \\
\hline GWA034012X & USGS-103 & 681.9 & Manganese & 48.5 & & & & UG/L & 07/01/2009 & & HCJ-172-09 \\
\hline GWA034012X & USGS-103 & 681.9 & Mercury & 0.066 & & $U$ & & UG/L & 07/01/2009 & & HCJ-172-09 \\
\hline GWA034012X & USGS-103 & 681.9 & Nickel & 1.02 & & B & & UG/L & 07/01/2009 & & HCJ-172-09 \\
\hline GWA034012X & USGS-103 & 681.9 & Potassium & 2990 & & B & & UG/L & 07/01/2009 & & HCJ-172-09 \\
\hline GWA034012X & USGS-103 & 681.9 & Selenium & 1 & & $U$ & & UG/L & 07/01/2009 & & HCJ-172-09 \\
\hline GWA034012X & USGS-103 & 681.9 & Silver & 0.2 & & $\mathrm{U}$ & & UG/L & 07/01/2009 & & HCJ-172-09 \\
\hline GWA034012X & USGS-103 & 681.9 & Sodium & 15400 & & $\mathrm{E}$ & $\mathrm{J}$ & UG/L & 07/01/2009 & & HCJ-172-09 \\
\hline GWA034012X & USGS-103 & 681.9 & Strontium & 143 & & & & UG/L & 07/01/2009 & & HCJ-172-09 \\
\hline GWA034012X & USGS-103 & 681.9 & Thallium & 0.3 & & U & & UG/L & 07/01/2009 & & HCJ-172-09 \\
\hline GWA034012X & USGS-103 & 681.9 & Uranium & 0.585 & & B & & UG/L & 07/01/2009 & & HCJ-172-09 \\
\hline GWA034012X & USGS-103 & 681.9 & Vanadium & 3 & & U & & UG/L & 07/01/2009 & & HCJ-172-09 \\
\hline GWA034012X & USGS-103 & 681.9 & Zinc & 36.1 & & & & UG/L & 07/01/2009 & & HCJ-172-09 \\
\hline GWA035012X & USGS-103 & 805.2 & Aluminum & 15 & & $\mathrm{U}$ & & UG/L & 07/01/2009 & & HCJ-172-09 \\
\hline GWA035012X & USGS-103 & 805.2 & Antimony & 1 & & $U$ & & UG/L & 07/01/2009 & & HCJ-172-09 \\
\hline GWA035012X & USGS-103 & 805.2 & Arsenic & 1.95 & & B & & UG/L & 07/01/2009 & & HCJ-172-09 \\
\hline GWA035012X & USGS-103 & 805.2 & Barium & 30.5 & & B & & UG/L & 07/01/2009 & & HCJ-172-09 \\
\hline GWA035012X & USGS-103 & 805.2 & Beryllium & 0.1 & & $U$ & & UG/L & 07/01/2009 & & HCJ-172-09 \\
\hline GWA035012X & USGS-103 & 805.2 & Cadmium & 0.11 & & U & & UG/L & 07/01/2009 & & HCJ-172-09 \\
\hline GWA035012X & USGS-103 & 805.2 & Calcium & 32600 & & & & UG/L & 07/01/2009 & & HCJ-172-09 \\
\hline GWA035012X & USGS-103 & 805.2 & Chromium & 4.97 & & B & & UG/L & 07/01/2009 & & HCJ-172-09 \\
\hline GWA035012X & USGS-103 & 805.2 & Cobalt & 0.109 & & B & & UG/L & 07/01/2009 & & HCJ-172-09 \\
\hline GWA035012X & USGS-103 & 805.2 & Copper & 0.982 & & B & & UG/L & 07/01/2009 & & HCJ-172-09 \\
\hline GWA035012X & USGS-103 & 805.2 & Iron & 195 & & & & UG/L & 07/01/2009 & & HCJ-172-09 \\
\hline GWA035012X & USGS-103 & 805.2 & Lead & 0.5 & & $U$ & & UG/L & 07/01/2009 & & HCJ-172-09 \\
\hline GWA035012X & USGS-103 & 805.2 & Magnesium & 14400 & & & & UG/L & 07/01/2009 & & HCJ-172-09 \\
\hline GWA035012X & USGS-103 & 805.2 & Manganese & 1.74 & & B & & UG/L & 07/01/2009 & & HCJ-172-09 \\
\hline
\end{tabular}


Table A-2. (continued).

\begin{tabular}{|c|c|c|c|c|c|c|c|c|c|c|c|}
\hline $\begin{array}{c}\text { Field Sample } \\
\text { Number }\end{array}$ & Location & Depth & Compound & $\begin{array}{l}\text { Sample } \\
\text { Result }\end{array}$ & $\begin{array}{l}\text { Sample } \\
\text { Error }\end{array}$ & $\begin{array}{c}\text { Result } \\
\text { Qualifier }\end{array}$ & $\begin{array}{l}\text { Validation } \\
\text { Flag }\end{array}$ & $\begin{array}{c}\text { Sample } \\
\text { Units }\end{array}$ & $\begin{array}{c}\text { Date Sample } \\
\text { Collected }\end{array}$ & MDA & L\&V Report Number \\
\hline GWA035012X & USGS-103 & 805.2 & Mercury & 0.066 & & $U$ & & UG/L & 07/01/2009 & & HCJ-172-09 \\
\hline GWA035012X & USGS-103 & 805.2 & Nickel & 1.17 & & B & & UG/L & 07/01/2009 & & HCJ-172-09 \\
\hline GWA035012X & USGS-103 & 805.2 & Potassium & 2490 & & B & & UG/L & 07/01/2009 & & HCJ-172-09 \\
\hline GWA035012X & USGS-103 & 805.2 & Selenium & 1.33 & & B & & UG/L & 07/01/2009 & & HCJ-172-09 \\
\hline GWA035012X & USGS-103 & 805.2 & Silver & 0.2 & & U & & UG/L & 07/01/2009 & & HCJ-172-09 \\
\hline GWA035012X & USGS-103 & 805.2 & Sodium & 13700 & & E & $\mathrm{J}$ & UG/L & 07/01/2009 & & HCJ-172-09 \\
\hline GWA035012X & USGS-103 & 805.2 & Strontium & 179 & & & & UG/L & 07/01/2009 & & HCJ-172-09 \\
\hline GWA035012X & USGS-103 & 805.2 & Thallium & 0.3 & & U & & UG/L & 07/01/2009 & & HCJ-172-09 \\
\hline GWA035012X & USGS-103 & 805.2 & Uranium & 2.25 & & B & & UG/L & 07/01/2009 & & HCJ-172-09 \\
\hline GWA035012X & USGS-103 & 805.2 & Vanadium & 7.63 & & B & & UG/L & 07/01/2009 & & HCJ-172-09 \\
\hline GWA035012X & USGS-103 & 805.2 & Zinc & 45.2 & & & & UG/L & 07/01/2009 & & HCJ-172-09 \\
\hline GWA036012X & USGS-103 & 913.8 & Aluminum & 15 & & U & & UG/L & 07/02/2009 & & HCJ-172-09 \\
\hline GWA036012X & USGS-103 & 913.8 & Antimony & 1 & & U & & UG/L & 07/02/2009 & & HCJ-172-09 \\
\hline GWA036012X & USGS-103 & 913.8 & Arsenic & 1.6 & & $U$ & & UG/L & 07/02/2009 & & HCJ-172-09 \\
\hline GWA036012X & USGS-103 & 913.8 & Barium & 28.7 & & B & & UG/L & 07/02/2009 & & HCJ-172-09 \\
\hline GWA036012X & USGS-103 & 913.8 & Beryllium & 0.1 & & U & & UG/L & 07/02/2009 & & HCJ-172-09 \\
\hline GWA036012X & USGS-103 & 913.8 & Cadmium & 0.11 & & U & & UG/L & 07/02/2009 & & HCJ-172-09 \\
\hline GWA036012X & USGS-103 & 913.8 & Calcium & 30500 & & & & UG/L & 07/02/2009 & & HCJ-172-09 \\
\hline GWA036012X & USGS-103 & 913.8 & Chromium & 5.44 & & & & UG/L & 07/02/2009 & & HCJ-172-09 \\
\hline GWA036012X & USGS-103 & 913.8 & Cobalt & 0.1 & & $\mathrm{U}$ & & UG/L & 07/02/2009 & & HCJ-172-09 \\
\hline GWA036012X & USGS-103 & 913.8 & Copper & 0.755 & & B & & UG/L & 07/02/2009 & & HCJ-172-09 \\
\hline GWA036012X & USGS-103 & 913.8 & Iron & 165 & & & & UG/L & 07/02/2009 & & HCJ-172-09 \\
\hline GWA036012X & USGS-103 & 913.8 & Lead & 0.5 & & $U$ & & UG/L & 07/02/2009 & & HCJ-172-09 \\
\hline GWA036012X & USGS-103 & 913.8 & Magnesium & 14100 & & & & UG/L & 07/02/2009 & & HCJ-172-09 \\
\hline GWA036012X & USGS-103 & 913.8 & Manganese & 1.18 & & B & & UG/L & 07/02/2009 & & HCJ-172-09 \\
\hline GWA036012X & USGS-103 & 913.8 & Mercury & 0.066 & & U & & UG/L & 07/02/2009 & & HCJ-172-09 \\
\hline GWA036012X & USGS-103 & 913.8 & Nickel & 0.985 & & B & & UG/L & 07/02/2009 & & HCJ-172-09 \\
\hline GWA036012X & USGS-103 & 913.8 & Potassium & 2470 & & B & & UG/L & 07/02/2009 & & HCJ-172-09 \\
\hline GWA036012X & USGS-103 & 913.8 & Selenium & 1 & & U & & UG/L & 07/02/2009 & & HCJ-172-09 \\
\hline GWA036012X & USGS-103 & 913.8 & Silver & 0.2 & & U & & UG/L & 07/02/2009 & & HCJ-172-09 \\
\hline GWA036012X & USGS-103 & 913.8 & Sodium & 9600 & & E & $\mathrm{J}$ & UG/L & 07/02/2009 & & HCJ-172-09 \\
\hline GWA036012X & USGS-103 & 913.8 & Strontium & 171 & & & & UG/L & 07/02/2009 & & HCJ-172-09 \\
\hline GWA036012X & USGS-103 & 913.8 & Thallium & 0.3 & & U & & UG/L & 07/02/2009 & & HCJ-172-09 \\
\hline GWA036012X & USGS-103 & 913.8 & Uranium & 2 & & B & & UG/L & 07/02/2009 & & HCJ-172-09 \\
\hline GWA036012X & USGS-103 & 913.8 & Vanadium & 10.4 & & B & & UG/L & 07/02/2009 & & HCJ-172-09 \\
\hline GWA036012X & USGS-103 & 913.8 & Zinc & 38.7 & & & & UG/L & 07/02/2009 & & HCJ-172-09 \\
\hline GWA037012X & USGS-103 & 999.4 & Aluminum & 15 & & U & & UG/L & 07/07/2009 & & HCJ-172-09 \\
\hline GWA037012X & USGS-103 & 999.4 & Antimony & 1 & & $U$ & & UG/L & 07/07/2009 & & HCJ-172-09 \\
\hline GWA037012X & USGS-103 & 999.4 & Arsenic & 1.6 & & $U$ & & UG/L & 07/07/2009 & & HCJ-172-09 \\
\hline GWA037012X & USGS-103 & 999.4 & Barium & 41.1 & & B & & UG/L & 07/07/2009 & & HCJ-172-09 \\
\hline GWA037012X & USGS-103 & 999.4 & Beryllium & 0.1 & & $\mathrm{U}$ & & UG/L & 07/07/2009 & & HCJ-172-09 \\
\hline GWA037012X & USGS-103 & 999.4 & Cadmium & 0.11 & & $U$ & & UG/L & 07/07/2009 & & HCJ-172-09 \\
\hline GWA037012X & USGS-103 & 999.4 & Calcium & 35400 & & & & UG/L & 07/07/2009 & & HCJ-172-09 \\
\hline GWA037012X & USGS-103 & 999.4 & Chromium & 5.81 & & & & UG/L & 07/07/2009 & & HCJ-172-09 \\
\hline
\end{tabular}


Table A-2. (continued).

\begin{tabular}{|c|c|c|c|c|c|c|c|c|c|c|c|}
\hline $\begin{array}{c}\text { Field Sample } \\
\text { Number }\end{array}$ & Location & Depth & Compound & $\begin{array}{l}\text { Sample } \\
\text { Result }\end{array}$ & $\begin{array}{c}\text { Sample } \\
\text { Error }\end{array}$ & $\begin{array}{c}\text { Result } \\
\text { Qualifier }\end{array}$ & $\begin{array}{c}\text { Validation } \\
\text { Flag }\end{array}$ & $\begin{array}{c}\text { Sample } \\
\text { Units }\end{array}$ & $\begin{array}{c}\text { Date Sample } \\
\text { Collected }\end{array}$ & MDA & L\&V Report Number \\
\hline GWA037012X & USGS-103 & 999.4 & Cobalt & 0.103 & & $\mathrm{~B}$ & & UG/L & 07/07/2009 & & HCJ-172-09 \\
\hline GWA037012X & USGS-103 & 999.4 & Copper & 0.936 & & B & & UG/L & 07/07/2009 & & HCJ-172-09 \\
\hline GWA037012X & USGS-103 & 999.4 & Iron & 200 & & & & UG/L & 07/07/2009 & & HCJ-172-09 \\
\hline GWA037012X & USGS-103 & 999.4 & Lead & 0.5 & & $\mathrm{U}$ & & UG/L & 07/07/2009 & & HCJ-172-09 \\
\hline GWA037012X & USGS-103 & 999.4 & Magnesium & 16600 & & & & UG/L & 07/07/2009 & & HCJ-172-09 \\
\hline GWA037012X & USGS-103 & 999.4 & Manganese & 1 & & U & & UG/L & 07/07/2009 & & HCJ-172-09 \\
\hline GWA037012X & USGS-103 & 999.4 & Mercury & 0.066 & & U & & UG/L & 07/07/2009 & & HCJ-172-09 \\
\hline GWA037012X & USGS-103 & 999.4 & Nickel & 1.17 & & B & & UG/L & 07/07/2009 & & HCJ-172-09 \\
\hline GWA037012X & USGS-103 & 999.4 & Potassium & 2260 & & B & & UG/L & 07/07/2009 & & HCJ-172-09 \\
\hline GWA037012X & USGS-103 & 999.4 & Selenium & 1.25 & & B & & UG/L & 07/07/2009 & & HCJ-172-09 \\
\hline GWA037012X & USGS-103 & 999.4 & Silver & 0.2 & & U & & UG/L & 07/07/2009 & & HCJ-172-09 \\
\hline GWA037012X & USGS-103 & 999.4 & Sodium & 10000 & & $\mathrm{E}$ & $\mathrm{J}$ & UG/L & 07/07/2009 & & HCJ-172-09 \\
\hline GWA037012X & USGS-103 & 999.4 & Strontium & 182 & & & & UG/L & 07/07/2009 & & HCJ-172-09 \\
\hline GWA037012X & USGS-103 & 999.4 & Thallium & 0.3 & & $\mathrm{U}$ & & UG/L & 07/07/2009 & & HCJ-172-09 \\
\hline GWA037012X & USGS-103 & 999.4 & Uranium & 1.71 & & B & & UG/L & 07/07/2009 & & HCJ-172-09 \\
\hline GWA037012X & USGS-103 & 999.4 & Vanadium & 3.7 & & B & & UG/L & 07/07/2009 & & HCJ-172-09 \\
\hline GWA037012X & USGS-103 & 999.4 & Zinc & 20.9 & & & & UG/L & 07/07/2009 & & HCJ-172-09 \\
\hline GWA038012X & USGS-103 & 1095.1 & Aluminum & 15 & & U & & UG/L & 07/08/2009 & & HCJ-172-09 \\
\hline GWA038012X & USGS-103 & 1095.1 & Antimony & 1 & & $U$ & & UG/L & 07/08/2009 & & HCJ-172-09 \\
\hline GWA038012X & USGS-103 & 1095.1 & Arsenic & 1.61 & & B & & UG/L & 07/08/2009 & & HCJ-172-09 \\
\hline GWA038012X & USGS-103 & 1095.1 & Barium & 45.8 & & B & & UG/L & 07/08/2009 & & HCJ-172-09 \\
\hline GWA038012X & USGS-103 & 1095.1 & Beryllium & 0.1 & & U & & UG/L & 07/08/2009 & & HCJ-172-09 \\
\hline GWA038012X & USGS-103 & 1095.1 & Cadmium & 0.11 & & U & & UG/L & 07/08/2009 & & HCJ-172-09 \\
\hline GWA038012X & USGS-103 & 1095.1 & Calcium & 40800 & & & & UG/L & 07/08/2009 & & HCJ-172-09 \\
\hline GWA038012X & USGS-103 & 1095.1 & Chromium & 5.99 & & & & UG/L & 07/08/2009 & & HCJ-172-09 \\
\hline GWA038012X & USGS-103 & 1095.1 & Cobalt & 0.198 & & B & & UG/L & 07/08/2009 & & HCJ-172-09 \\
\hline GWA038012X & USGS-103 & 1095.1 & Copper & 0.77 & & B & & UG/L & 07/08/2009 & & HCJ-172-09 \\
\hline GWA038012X & USGS-103 & 1095.1 & Iron & 240 & & & & UG/L & 07/08/2009 & & HCJ-172-09 \\
\hline GWA038012X & USGS-103 & 1095.1 & Lead & 0.5 & & U & & UG/L & 07/08/2009 & & HCJ-172-09 \\
\hline GWA038012X & USGS-103 & 1095.1 & Magnesium & 17000 & & & & UG/L & 07/08/2009 & & HCJ-172-09 \\
\hline GWA038012X & USGS-103 & 1095.1 & Manganese & 1 & & $U$ & & UG/L & 07/08/2009 & & HCJ-172-09 \\
\hline GWA038012X & USGS-103 & 1095.1 & Mercury & 0.066 & & U & & UG/L & 07/08/2009 & & HCJ-172-09 \\
\hline GWA038012X & USGS-103 & 1095.1 & Nickel & 1.24 & & B & & UG/L & 07/08/2009 & & HCJ-172-09 \\
\hline GWA038012X & USGS-103 & 1095.1 & Potassium & 2350 & & B & & UG/L & 07/08/2009 & & HCJ-172-09 \\
\hline GWA038012X & USGS-103 & 1095.1 & Selenium & 1 & & U & & UG/L & 07/08/2009 & & HCJ-172-09 \\
\hline GWA038012X & USGS-103 & 1095.1 & Silver & 0.2 & & U & & UG/L & 07/08/2009 & & HCJ-172-09 \\
\hline GWA038012X & USGS-103 & 1095.1 & Sodium & 10300 & & $\mathrm{E}$ & $\mathrm{J}$ & UG/L & 07/08/2009 & & HCJ-172-09 \\
\hline GWA038012X & USGS-103 & 1095.1 & Strontium & 199 & & & & UG/L & 07/08/2009 & & HCJ-172-09 \\
\hline GWA038012X & USGS-103 & 1095.1 & Thallium & 0.3 & & U & & UG/L & 07/08/2009 & & HCJ-172-09 \\
\hline GWA038012X & USGS-103 & 1095.1 & Uranium & 1.95 & & B & & UG/L & 07/08/2009 & & HCJ-172-09 \\
\hline GWA038012X & USGS-103 & 1095.1 & Vanadium & 6.15 & & B & & UG/L & 07/08/2009 & & HCJ-172-09 \\
\hline GWA038012X & USGS-103 & 1095.1 & Zinc & 24.1 & & & & UG/L & 07/08/2009 & & HCJ-172-09 \\
\hline GWA039012X & USGS-103 & 1220.2 & Aluminum & 15 & & U & & UG/L & 07/09/2009 & & HCJ-172-09 \\
\hline GWA039012X & USGS-103 & 1220.2 & Antimony & 1 & & $U$ & & UG/L & 07/09/2009 & & HCJ-172-09 \\
\hline
\end{tabular}


Table A-2. (continued).

\begin{tabular}{|c|c|c|c|c|c|c|c|c|c|c|c|}
\hline $\begin{array}{c}\text { Field Sample } \\
\text { Number }\end{array}$ & Location & Depth & Compound & $\begin{array}{l}\text { Sample } \\
\text { Result }\end{array}$ & $\begin{array}{c}\text { Sample } \\
\text { Error }\end{array}$ & $\begin{array}{c}\text { Result } \\
\text { Qualifier }\end{array}$ & $\begin{array}{c}\text { Validation } \\
\text { Flag }\end{array}$ & $\begin{array}{c}\text { Sample } \\
\text { Units }\end{array}$ & $\begin{array}{c}\text { Date Sample } \\
\text { Collected }\end{array}$ & MDA & L\&V Report Number \\
\hline GWA039012X & USGS-103 & 1220.2 & Arsenic & 1.6 & & $\mathrm{U}$ & & UG/L & 07/09/2009 & & HCJ-172-09 \\
\hline GWA039012X & USGS-103 & 1220.2 & Barium & 44.6 & & $\mathrm{~B}$ & & UG/L & 07/09/2009 & & HCJ-172-09 \\
\hline GWA039012X & USGS-103 & 1220.2 & Beryllium & 0.1 & & $U$ & & UG/L & 07/09/2009 & & HCJ-172-09 \\
\hline GWA039012X & USGS-103 & 1220.2 & Cadmium & 0.11 & & $U$ & & UG/L & $07 / 09 / 2009$ & & HCJ-172-09 \\
\hline GWA039012X & USGS-103 & 1220.2 & Calcium & 39700 & & & & UG/L & 07/09/2009 & & HCJ-172-09 \\
\hline GWA039012X & USGS-103 & 1220.2 & Chromium & 5.57 & & & & UG/L & $07 / 09 / 2009$ & & HCJ-172-09 \\
\hline GWA039012X & USGS-103 & 1220.2 & Cobalt & 0.246 & & $\mathrm{~B}$ & & UG/L & $07 / 09 / 2009$ & & HCJ-172-09 \\
\hline GWA039012X & USGS-103 & 1220.2 & Copper & 0.732 & & B & & UG/L & 07/09/2009 & & HCJ-172-09 \\
\hline GWA039012X & USGS-103 & 1220.2 & Iron & 221 & & & & UG/L & 07/09/2009 & & HCJ-172-09 \\
\hline GWA039012X & USGS-103 & 1220.2 & Lead & 0.5 & & $U$ & & UG/L & 07/09/2009 & & HCJ-172-09 \\
\hline GWA039012X & USGS-103 & 1220.2 & Magnesium & 18100 & & & & UG/L & $07 / 09 / 2009$ & & HCJ-172-09 \\
\hline GWA039012X & USGS-103 & 1220.2 & Manganese & 1 & & $U$ & & UG/L & 07/09/2009 & & HCJ-172-09 \\
\hline GWA039012X & USGS-103 & 1220.2 & Mercury & 0.066 & & U & & UG/L & 07/09/2009 & & HCJ-172-09 \\
\hline GWA039012X & USGS-103 & 1220.2 & Nickel & 1.24 & & B & & UG/L & 07/09/2009 & & HCJ-172-09 \\
\hline GWA039012X & USGS-103 & 1220.2 & Potassium & 2400 & & B & & UG/L & 07/09/2009 & & HCJ-172-09 \\
\hline GWA039012X & USGS-103 & 1220.2 & Selenium & 1.39 & & B & & UG/L & 07/09/2009 & & HCJ-172-09 \\
\hline GWA039012X & USGS-103 & 1220.2 & Silver & 0.2 & & $U$ & & UG/L & 07/09/2009 & & HCJ-172-09 \\
\hline GWA039012X & USGS-103 & 1220.2 & Sodium & 10400 & & $E$ & $\mathrm{~J}$ & UG/L & 07/09/2009 & & HCJ-172-09 \\
\hline GWA039012X & USGS-103 & 1220.2 & Strontium & 194 & & & & UG/L & 07/09/2009 & & HCJ-172-09 \\
\hline GWA039012X & USGS-103 & 1220.2 & Thallium & 0.3 & & U & & UG/L & 07/09/2009 & & HCJ-172-09 \\
\hline GWA039012X & USGS-103 & 1220.2 & Uranium & 1.9 & & B & & UG/L & $07 / 09 / 2009$ & & HCJ-172-09 \\
\hline GWA039012X & USGS-103 & 1220.2 & Vanadium & 4.86 & & B & & UG/L & 07/09/2009 & & HCJ-172-09 \\
\hline GWA039012X & USGS-103 & 1220.2 & Zinc & 35.8 & & & & UG/L & 07/09/2009 & & HCJ-172-09 \\
\hline GWA040012X & USGS-103 & 1269.4 & Aluminum & 15 & & $U$ & & UG/L & 07/13/2009 & & HCJ-172-09 \\
\hline GWA040012X & USGS-103 & 1269.4 & Antimony & 1 & & U & & UG/L & 07/13/2009 & & HCJ-172-09 \\
\hline GWA040012X & USGS-103 & 1269.4 & Arsenic & 5.37 & & & $U$ & UG/L & $07 / 13 / 2009$ & & HCJ-172-09 \\
\hline GWA040012X & USGS-103 & 1269.4 & Barium & 45.4 & & B & & UG/L & $07 / 13 / 2009$ & & HCJ-172-09 \\
\hline GWA040012X & USGS-103 & 1269.4 & Beryllium & 0.1 & & $U$ & & UG/L & $07 / 13 / 2009$ & & HCJ-172-09 \\
\hline GWA040012X & USGS-103 & 1269.4 & Cadmium & 0.11 & & $U$ & & UG/L & 07/13/2009 & & HCJ-172-09 \\
\hline GWA040012X & USGS-103 & 1269.4 & Calcium & 41200 & & & & UG/L & 07/13/2009 & & HCJ-172-09 \\
\hline GWA040012X & USGS-103 & 1269.4 & Chromium & 6.89 & & & & UG/L & $07 / 13 / 2009$ & & HCJ-172-09 \\
\hline GWA040012X & USGS-103 & 1269.4 & Cobalt & 0.1 & & U & & UG/L & $07 / 13 / 2009$ & & HCJ-172-09 \\
\hline GWA040012X & USGS-103 & 1269.4 & Copper & 0.455 & & B & & UG/L & 07/13/2009 & & HCJ-172-09 \\
\hline GWA040012X & USGS-103 & 1269.4 & Iron & 65.7 & & B & & UG/L & $07 / 13 / 2009$ & & HCJ-172-09 \\
\hline GWA040012X & USGS-103 & 1269.4 & Lead & 0.5 & & $U$ & & UG/L & 07/13/2009 & & HCJ-172-09 \\
\hline GWA040012X & USGS-103 & 1269.4 & Magnesium & 15000 & & & & UG/L & 07/13/2009 & & HCJ-172-09 \\
\hline GWA040012X & USGS-103 & 1269.4 & Manganese & 1 & & $U$ & & UG/L & $07 / 13 / 2009$ & & HCJ-172-09 \\
\hline GWA040012X & USGS-103 & 1269.4 & Mercury & 0.066 & & U & & UG/L & $07 / 13 / 2009$ & & HCJ-172-09 \\
\hline GWA040012X & USGS-103 & 1269.4 & Nickel & 1.06 & & B & & UG/L & $07 / 13 / 2009$ & & HCJ-172-09 \\
\hline GWA040012X & USGS-103 & 1269.4 & Potassium & 2570 & & B & & UG/L & 07/13/2009 & & HCJ-172-09 \\
\hline GWA040012X & USGS-103 & 1269.4 & Selenium & 1.07 & & B & & UG/L & 07/13/2009 & & HCJ-172-09 \\
\hline GWA040012X & USGS-103 & 1269.4 & Silver & 0.2 & & $U$ & & UG/L & 07/13/2009 & & HCJ-172-09 \\
\hline GWA040012X & USGS-103 & 1269.4 & Sodium & 9620 & & $\mathrm{E}$ & & UG/L & 07/13/2009 & & HCJ-172-09 \\
\hline GWA040012X & USGS-103 & 1269.4 & Strontium & 220 & & & & UG/L & $07 / 13 / 2009$ & & HCJ-172-09 \\
\hline
\end{tabular}


Table A-2. (continued).

\begin{tabular}{|c|c|c|c|c|c|c|c|c|c|c|c|}
\hline $\begin{array}{c}\text { Field Sample } \\
\text { Number }\end{array}$ & Location & Depth & Compound & $\begin{array}{l}\text { Sample } \\
\text { Result }\end{array}$ & $\begin{array}{c}\text { Sample } \\
\text { Error }\end{array}$ & $\begin{array}{c}\text { Result } \\
\text { Qualifier }\end{array}$ & $\begin{array}{c}\text { Validation } \\
\text { Flag }\end{array}$ & $\begin{array}{c}\text { Sample } \\
\text { Units }\end{array}$ & $\begin{array}{c}\text { Date Sample } \\
\text { Collected }\end{array}$ & MDA & L\&V Report Number \\
\hline GWA040012X & USGS-103 & 1269.4 & Thallium & 0.375 & & $\mathrm{~B}$ & & UG/L & $07 / 13 / 2009$ & & HCJ-172-09 \\
\hline GWA040012X & USGS-103 & 1269.4 & Uranium & 1.84 & & B & & UG/L & $07 / 13 / 2009$ & & HCJ-172-09 \\
\hline GWA040012X & USGS-103 & 1269.4 & Vanadium & 3 & & $\mathrm{U}$ & & UG/L & 07/13/2009 & & HCJ-172-09 \\
\hline GWA040012X & USGS-103 & 1269.4 & Zinc & 21.3 & & & & UG/L & 07/13/2009 & & HCJ-172-09 \\
\hline GWA03401VL & USGS-103 & 681.9 & Dichlorodifluoromethane & 1 & & U & & UG/L & $07 / 01 / 2009$ & & HCJ-166-09 \\
\hline GWA03401VL & USGS-103 & 681.9 & Chloromethane & 1 & & $\mathrm{U}$ & & UG/L & 07/01/2009 & & HCJ-166-09 \\
\hline GWA03401VL & USGS-103 & 681.9 & Vinyl Chloride & 1 & & $\mathrm{U}$ & & UG/L & 07/01/2009 & & HCJ-166-09 \\
\hline GWA03401VL & USGS-103 & 681.9 & Bromomethane & 1 & & U & & UG/L & 07/01/2009 & & HCJ-166-09 \\
\hline GWA03401VL & USGS-103 & 681.9 & Chloroethane & 1 & & U & & UG/L & 07/01/2009 & & HCJ-166-09 \\
\hline GWA03401VL & USGS-103 & 681.9 & Trichlorofluoromethane & 1 & & $\mathrm{U}$ & & UG/L & 07/01/2009 & & HCJ-166-09 \\
\hline GWA03401VL & USGS-103 & 681.9 & Acetone & 5 & & $\mathrm{U}$ & & UG/L & $07 / 01 / 2009$ & & HCJ-166-09 \\
\hline GWA03401VL & USGS-103 & 681.9 & 1,1-Dichloroethene & 1 & & U & & UG/L & $07 / 01 / 2009$ & & HCJ-166-09 \\
\hline GWA03401VL & USGS-103 & 681.9 & Methyl acetate & 5 & & $u$ & & UG/L & $07 / 01 / 2009$ & & HCJ-166-09 \\
\hline GWA03401VL & USGS-103 & 681.9 & Methylene Chloride & 5 & & $\mathrm{u}$ & & UG/L & $07 / 01 / 2009$ & & HCJ-166-09 \\
\hline GWA03401VL & USGS-103 & 681.9 & Carbon disulfide & 5 & & U & & UG/L & $07 / 01 / 2009$ & & HCJ-166-09 \\
\hline GWA03401VL & USGS-103 & 681.9 & Tert-butyl methyl ether & 1 & & U & & UG/L & $07 / 01 / 2009$ & & HCJ-166-09 \\
\hline GWA03401VL & USGS-103 & 681.9 & trans-1,2-Dichloroethylene & 1 & & $\mathrm{u}$ & & UG/L & $07 / 01 / 2009$ & & HCJ-166-09 \\
\hline GWA03401VL & USGS-103 & 681.9 & 1,1-Dichloroethane & 1 & & $\mathrm{u}$ & & UG/L & 07/01/2009 & & HCJ-166-09 \\
\hline GWA03401VL & USGS-103 & 681.9 & 2-Butanone & 5 & & U & & UG/L & $07 / 01 / 2009$ & & HCJ-166-09 \\
\hline GWA03401VL & USGS-103 & 681.9 & cis-1,2-Dichloroethylene & 1 & & $u$ & & UG/L & $07 / 01 / 2009$ & & HCJ-166-09 \\
\hline GWA03401VL & USGS-103 & 681.9 & Chloroform & 1 & & $\mathrm{u}$ & & UG/L & 07/01/2009 & & HCJ-166-09 \\
\hline GWA03401VL & USGS-103 & 681.9 & 1,1,1-Trichloroethane & 1 & & U & & UG/L & 07/01/2009 & & HCJ-166-09 \\
\hline GWA03401VL & USGS-103 & 681.9 & Cyclohexane & 1 & & U & & UG/L & $07 / 01 / 2009$ & & HCJ-166-09 \\
\hline GWA03401VL & USGS-103 & 681.9 & Carbon tetrachloride & 1 & & $u$ & & UG/L & $07 / 01 / 2009$ & & HCJ-166-09 \\
\hline GWA03401VL & USGS-103 & 681.9 & 1,2-Dichloroethane & 1 & & $\mathrm{U}$ & & UG/L & 07/01/2009 & & HCJ-166-09 \\
\hline GWA03401VL & USGS-103 & 681.9 & Benzene & 1 & & U & & UG/L & 07/01/2009 & & HCJ-166-09 \\
\hline GWA03401VL & USGS-103 & 681.9 & Trichloroethylene & 1 & & $\mathrm{U}$ & & UG/L & 07/01/2009 & & HCJ-166-09 \\
\hline GWA03401VL & USGS-103 & 681.9 & 1,2-Dichloropropane & 1 & & $\mathrm{U}$ & & UG/L & $07 / 01 / 2009$ & & HCJ-166-09 \\
\hline GWA03401VL & USGS-103 & 681.9 & Methyl cyclohexane & 1 & & U & & UG/L & 07/01/2009 & & HCJ-166-09 \\
\hline GWA03401VL & USGS-103 & 681.9 & Bromodichloromethane & 1 & & U & & UG/L & 07/01/2009 & & HCJ-166-09 \\
\hline GWA03401VL & USGS-103 & 681.9 & 4-Methyl-2-pentanone & 5 & & $u$ & & UG/L & $07 / 01 / 2009$ & & HCJ-166-09 \\
\hline GWA03401VL & USGS-103 & 681.9 & cis-1,3-Dichloropropylene & 1 & & $\mathrm{U}$ & & UG/L & $07 / 01 / 2009$ & & HCJ-166-09 \\
\hline GWA03401VL & USGS-103 & 681.9 & Toluene & 1 & & U & & UG/L & $07 / 01 / 2009$ & & HCJ-166-09 \\
\hline GWA03401VL & USGS-103 & 681.9 & trans-1,3-Dichloropropylene & 1 & & $u$ & & UG/L & $07 / 01 / 2009$ & & HCJ-166-09 \\
\hline GWA03401VL & USGS-103 & 681.9 & 1,1,2-Trichloroethane & 1 & & $\mathrm{u}$ & & UG/L & 07/01/2009 & & HCJ-166-09 \\
\hline GWA03401VL & USGS-103 & 681.9 & 2-Hexanone & 5 & & U & & UG/L & 07/01/2009 & & HCJ-166-09 \\
\hline GWA03401VL & USGS-103 & 681.9 & Tetrachloroethylene & 1 & & BJ & $\mathrm{U}$ & UG/L & 07/01/2009 & & HCJ-166-09 \\
\hline GWA03401VL & USGS-103 & 681.9 & Dibromochloromethane & 1 & & $U$ & & UG/L & $07 / 01 / 2009$ & & HCJ-166-09 \\
\hline GWA03401VL & USGS-103 & 681.9 & 1,2-Dibromoethane & 1 & & $\mathrm{U}$ & & UG/L & $07 / 01 / 2009$ & & HCJ-166-09 \\
\hline GWA03401VL & USGS-103 & 681.9 & Chlorobenzene & 1 & & $\mathrm{U}$ & & UG/L & 07/01/2009 & & HCJ-166-09 \\
\hline GWA03401VL & USGS-103 & 681.9 & Ethylbenzene & 1 & & $\mathrm{U}$ & & UG/L & 07/01/2009 & & HCJ-166-09 \\
\hline GWA03401VL & USGS-103 & 681.9 & Styrene & 1 & & $\mathrm{U}$ & & UG/L & 07/01/2009 & & HCJ-166-09 \\
\hline GWA03401VL & USGS-103 & 681.9 & Bromoform & 1 & & $u$ & & UG/L & $07 / 01 / 2009$ & & HCJ-166-09 \\
\hline GWA03401VL & USGS-103 & 681.9 & 1,1,2,2-Tetrachloroethane & 1 & & U & & UG/L & $07 / 01 / 2009$ & & HCJ-166-09 \\
\hline
\end{tabular}


Table A-2. (continued).

\begin{tabular}{|c|c|c|c|c|c|c|c|c|c|c|c|}
\hline $\begin{array}{c}\text { Field Sample } \\
\text { Number }\end{array}$ & Location & Depth & Compound & $\begin{array}{c}\text { Sample } \\
\text { Result }\end{array}$ & $\begin{array}{c}\text { Sample } \\
\text { Error }\end{array}$ & $\begin{array}{c}\text { Result } \\
\text { Qualifier } \\
\end{array}$ & $\begin{array}{c}\text { Validation } \\
\text { Flag } \\
\end{array}$ & $\begin{array}{c}\text { Sample } \\
\text { Units }\end{array}$ & $\begin{array}{c}\text { Date Sample } \\
\text { Collected }\end{array}$ & MDA & L\&V Report Number \\
\hline GWA03401VL & USGS-103 & 681.9 & Isopropylbenzene & 1 & & $U$ & & UG/L & $07 / 01 / 2009$ & & HCJ-166-09 \\
\hline GWA03401VL & USGS-103 & 681.9 & 1,3-Dichlorobenzene & 1 & & U & & UG/L & $07 / 01 / 2009$ & & HCJ-166-09 \\
\hline GWA03401VL & USGS-103 & 681.9 & 1,4-Dichlorobenzene & 1 & & $\mathrm{u}$ & & UG/L & 07/01/2009 & & HCJ-166-09 \\
\hline GWA03401VL & USGS-103 & 681.9 & 1,2-Dibromo-3-chloropropane & 1 & & $\mathrm{U}$ & & UG/L & 07/01/2009 & & HCJ-166-09 \\
\hline GWA03401VL & USGS-103 & 681.9 & Trichlorotrifluoroethane & 5 & & U & & UG/L & $07 / 01 / 2009$ & & HCJ-166-09 \\
\hline GWA03401VL & USGS-103 & 681.9 & Xylene (Total) & 1 & & $\mathrm{u}$ & & UG/L & 07/01/2009 & & HCJ-166-09 \\
\hline GWA03401VL & USGS-103 & 681.9 & 1,2,4-Trichlorobenzene & 1 & & $\mathrm{U}$ & & UG/L & 07/01/2009 & & HCJ-166-09 \\
\hline GWA03401VL & USGS-103 & 681.9 & 1,2-Dichlorobenzene & 1 & & U & & UG/L & $07 / 01 / 2009$ & & HCJ-166-09 \\
\hline GWA03401VL & USGS-103 & 681.9 & Unknown & 25.1 & & $\mathrm{~J}$ & & UG/L & $07 / 01 / 2009$ & & \\
\hline GWA03501VL & USGS-103 & 805.2 & Dichlorodifluoromethane & 1 & & $\mathrm{u}$ & & UG/L & 07/01/2009 & & HCJ-166-09 \\
\hline GWA03501VL & USGS-103 & 805.2 & Chloromethane & 1 & & $\mathrm{U}$ & & UG/L & 07/01/2009 & & HCJ-166-09 \\
\hline GWA03501VL & USGS-103 & 805.2 & Vinyl Chloride & 1 & & $U$ & & UG/L & $07 / 01 / 2009$ & & HCJ-166-09 \\
\hline GWA03501VL & USGS-103 & 805.2 & Bromomethane & 1 & & U & & UG/L & $07 / 01 / 2009$ & & HCJ-166-09 \\
\hline GWA03501VL & USGS-103 & 805.2 & Chloroethane & 1 & & $\mathrm{U}$ & & UG/L & $07 / 01 / 2009$ & & HCJ-166-09 \\
\hline GWA03501VL & USGS-103 & 805.2 & Trichlorofluoromethane & 1 & & U & & UG/L & 07/01/2009 & & HCJ-166-09 \\
\hline GWA03501VL & USGS-103 & 805.2 & Acetone & 5 & & U & & UG/L & $07 / 01 / 2009$ & & HCJ-166-09 \\
\hline GWA03501VL & USGS-103 & 805.2 & 1,1-Dichloroethene & 1 & & $\mathrm{U}$ & & UG/L & $07 / 01 / 2009$ & & HCJ-166-09 \\
\hline GWA03501VL & USGS-103 & 805.2 & Methyl acetate & 5 & & $\mathrm{U}$ & & UG/L & 07/01/2009 & & HCJ-166-09 \\
\hline GWA03501VL & USGS-103 & 805.2 & Methylene Chloride & 5 & & U & & UG/L & 07/01/2009 & & HCJ-166-09 \\
\hline GWA03501VL & USGS-103 & 805.2 & Carbon disulfide & 5 & & $u$ & & UG/L & $07 / 01 / 2009$ & & HCJ-166-09 \\
\hline GWA03501VL & USGS-103 & 805.2 & Tert-butyl methyl ether & 1 & & $\mathrm{U}$ & & UG/L & 07/01/2009 & & HCJ-166-09 \\
\hline GWA03501VL & USGS-103 & 805.2 & trans-1,2-Dichloroethylene & 1 & & U & & UG/L & 07/01/2009 & & HCJ-166-09 \\
\hline GWA03501VL & USGS-103 & 805.2 & 1,1-Dichloroethane & 1 & & U & & UG/L & $07 / 01 / 2009$ & & HCJ-166-09 \\
\hline GWA03501VL & USGS-103 & 805.2 & 2-Butanone & 5 & & U & & UG/L & $07 / 01 / 2009$ & & HCJ-166-09 \\
\hline GWA03501VL & USGS-103 & 805.2 & cis-1,2-Dichloroethylene & 1 & & $\mathrm{U}$ & & UG/L & 07/01/2009 & & HCJ-166-09 \\
\hline GWA03501VL & USGS-103 & 805.2 & Chloroform & 1 & & U & & UG/L & 07/01/2009 & & HCJ-166-09 \\
\hline GWA03501VL & USGS-103 & 805.2 & 1,1,1-Trichloroethane & 1 & & $\mathrm{U}$ & & UG/L & 07/01/2009 & & HCJ-166-09 \\
\hline GWA03501VL & USGS-103 & 805.2 & Cyclohexane & 1 & & $\mathrm{U}$ & & UG/L & $07 / 01 / 2009$ & & HCJ-166-09 \\
\hline GWA03501VL & USGS-103 & 805.2 & Carbon tetrachloride & 1 & & U & & UG/L & 07/01/2009 & & HCJ-166-09 \\
\hline GWA03501VL & USGS-103 & 805.2 & 1,2-Dichloroethane & 1 & & U & & UG/L & 07/01/2009 & & HCJ-166-09 \\
\hline GWA03501VL & USGS-103 & 805.2 & Benzene & 1 & & $u$ & & UG/L & $07 / 01 / 2009$ & & HCJ-166-09 \\
\hline GWA03501VL & USGS-103 & 805.2 & Trichloroethylene & 1 & & $\mathrm{U}$ & & UG/L & $07 / 01 / 2009$ & & HCJ-166-09 \\
\hline GWA03501VL & USGS-103 & 805.2 & 1,2-Dichloropropane & 1 & & U & & UG/L & $07 / 01 / 2009$ & & HCJ-166-09 \\
\hline GWA03501VL & USGS-103 & 805.2 & Methyl cyclohexane & 1 & & $U$ & & UG/L & 07/01/2009 & & HCJ-166-09 \\
\hline GWA03501VL & USGS-103 & 805.2 & Bromodichloromethane & 1 & & $\mathrm{u}$ & & UG/L & 07/01/2009 & & HCJ-166-09 \\
\hline GWA03501VL & USGS-103 & 805.2 & 4-Methyl-2-pentanone & 5 & & $\mathrm{U}$ & & UG/L & 07/01/2009 & & HCJ-166-09 \\
\hline GWA03501VL & USGS-103 & 805.2 & cis-1,3-Dichloropropylene & 1 & & U & & UG/L & 07/01/2009 & & HCJ-166-09 \\
\hline GWA03501VL & USGS-103 & 805.2 & Toluene & 1 & & $u$ & & UG/L & $07 / 01 / 2009$ & & HCJ-166-09 \\
\hline GWA03501VL & USGS-103 & 805.2 & trans-1,3-Dichloropropylene & 1 & & $\mathrm{U}$ & & UG/L & $07 / 01 / 2009$ & & HCJ-166-09 \\
\hline GWA03501VL & USGS-103 & 805.2 & 1,1,2-Trichloroethane & 1 & & $\mathrm{U}$ & & UG/L & 07/01/2009 & & HCJ-166-09 \\
\hline GWA03501VL & USGS-103 & 805.2 & 2-Hexanone & 5 & & $\mathrm{U}$ & & UG/L & 07/01/2009 & & HCJ-166-09 \\
\hline GWA03501VL & USGS-103 & 805.2 & Tetrachloroethylene & 1 & & BJ & $\mathrm{U}$ & UG/L & 07/01/2009 & & HCJ-166-09 \\
\hline GWA03501VL & USGS-103 & 805.2 & Dibromochloromethane & 1 & & $\mathrm{U}$ & & UG/L & 07/01/2009 & & HCJ-166-09 \\
\hline GWA03501VL & USGS-103 & 805.2 & 1,2-Dibromoethane & 1 & & $\mathrm{U}$ & & UG/L & $07 / 01 / 2009$ & & HCJ-166-09 \\
\hline
\end{tabular}


Table A-2. (continued).

\begin{tabular}{|c|c|c|c|c|c|c|c|c|c|c|c|}
\hline $\begin{array}{c}\text { Field Sample } \\
\text { Number }\end{array}$ & Location & Depth & Compound & $\begin{array}{c}\text { Sample } \\
\text { Result }\end{array}$ & $\begin{array}{c}\text { Sample } \\
\text { Error }\end{array}$ & $\begin{array}{c}\text { Result } \\
\text { Qualifier }\end{array}$ & $\begin{array}{c}\text { Validation } \\
\text { Flag }\end{array}$ & $\begin{array}{c}\text { Sample } \\
\text { Units }\end{array}$ & $\begin{array}{c}\text { Date Sample } \\
\text { Collected }\end{array}$ & MDA & L\&V Report Number \\
\hline GWA03501VL & USGS-103 & 805.2 & Chlorobenzene & 1 & & $U$ & & UG/L & $07 / 01 / 2009$ & & HCJ-166-09 \\
\hline GWA03501VL & USGS-103 & 805.2 & Ethylbenzene & 1 & & U & & UG/L & $07 / 01 / 2009$ & & HCJ-166-09 \\
\hline GWA03501VL & USGS-103 & 805.2 & Styrene & 1 & & $\mathrm{u}$ & & UG/L & 07/01/2009 & & HCJ-166-09 \\
\hline GWA03501VL & USGS-103 & 805.2 & Bromoform & 1 & & $\mathrm{u}$ & & UG/L & 07/01/2009 & & HCJ-166-09 \\
\hline GWA03501VL & USGS-103 & 805.2 & 1,1,2,2-Tetrachloroethane & 1 & & U & & UG/L & $07 / 01 / 2009$ & & HCJ-166-09 \\
\hline GWA03501VL & USGS-103 & 805.2 & Isopropylbenzene & 1 & & $\mathrm{u}$ & & UG/L & 07/01/2009 & & HCJ-166-09 \\
\hline GWA03501VL & USGS-103 & 805.2 & 1,3-Dichlorobenzene & 1 & & $\mathrm{U}$ & & UG/L & 07/01/2009 & & HCJ-166-09 \\
\hline GWA03501VL & USGS-103 & 805.2 & 1,4-Dichlorobenzene & 1 & & U & & UG/L & $07 / 01 / 2009$ & & HCJ-166-09 \\
\hline GWA03501VL & USGS-103 & 805.2 & 1,2-Dibromo-3-chloropropane & 1 & & U & & UG/L & $07 / 01 / 2009$ & & HCJ-166-09 \\
\hline GWA03501VL & USGS-103 & 805.2 & Trichlorotrifluoroethane & 5 & & $\mathrm{u}$ & & UG/L & 07/01/2009 & & HCJ-166-09 \\
\hline GWA03501VL & USGS-103 & 805.2 & Xylene (Total) & 1 & & $\mathrm{U}$ & & UG/L & $07 / 01 / 2009$ & & HCJ-166-09 \\
\hline GWA03501VL & USGS-103 & 805.2 & 1,2,4-Trichlorobenzene & 1 & & $U$ & & UG/L & 07/01/2009 & & HCJ-166-09 \\
\hline GWA03501VL & USGS-103 & 805.2 & 1,2-Dichlorobenzene & 1 & & U & & UG/L & $07 / 01 / 2009$ & & HCJ-166-09 \\
\hline GWA03501VL & USGS-103 & 805.2 & Unknown & 29.5 & & $\mathrm{~J}$ & & UG/L & $07 / 01 / 2009$ & & \\
\hline GWA03601VL & USGS-103 & 913.8 & Dichlorodifluoromethane & 1 & & U & & UG/L & $07 / 02 / 2009$ & & HCJ-166-09 \\
\hline GWA03601VL & USGS-103 & 913.8 & Chloromethane & 1 & & U & & UG/L & $07 / 02 / 2009$ & & HCJ-166-09 \\
\hline GWA03601VL & USGS-103 & 913.8 & Vinyl Chloride & 1 & & $\mathrm{U}$ & & UG/L & $07 / 02 / 2009$ & & HCJ-166-09 \\
\hline GWA03601VL & USGS-103 & 913.8 & Bromomethane & 1 & & $\mathrm{U}$ & & UG/L & $07 / 02 / 2009$ & & HCJ-166-09 \\
\hline GWA03601VL & USGS-103 & 913.8 & Chloroethane & 1 & & U & & UG/L & $07 / 02 / 2009$ & & HCJ-166-09 \\
\hline GWA03601VL & USGS-103 & 913.8 & Trichlorofluoromethane & 1 & & $u$ & & UG/L & $07 / 02 / 2009$ & & HCJ-166-09 \\
\hline GWA03601VL & USGS-103 & 913.8 & Acetone & 5 & & $\mathrm{U}$ & & UG/L & $07 / 02 / 2009$ & & HCJ-166-09 \\
\hline GWA03601VL & USGS-103 & 913.8 & 1,1-Dichloroethene & 1 & & U & & UG/L & $07 / 02 / 2009$ & & HCJ-166-09 \\
\hline GWA03601VL & USGS-103 & 913.8 & Methyl acetate & 5 & & U & & UG/L & $07 / 02 / 2009$ & & HCJ-166-09 \\
\hline GWA03601VL & USGS-103 & 913.8 & Methylene Chloride & 5 & & U & & UG/L & $07 / 02 / 2009$ & & HCJ-166-09 \\
\hline GWA03601VL & USGS-103 & 913.8 & Carbon disulfide & 5 & & $\mathrm{U}$ & & UG/L & $07 / 02 / 2009$ & & HCJ-166-09 \\
\hline GWA03601VL & USGS-103 & 913.8 & Tert-butyl methyl ether & 1 & & U & & UG/L & $07 / 02 / 2009$ & & HCJ-166-09 \\
\hline GWA03601VL & USGS-103 & 913.8 & trans-1,2-Dichloroethylene & 1 & & $\mathrm{U}$ & & UG/L & $07 / 02 / 2009$ & & HCJ-166-09 \\
\hline GWA03601VL & USGS-103 & 913.8 & 1,1-Dichloroethane & 1 & & $\mathrm{U}$ & & UG/L & $07 / 02 / 2009$ & & HCJ-166-09 \\
\hline GWA03601VL & USGS-103 & 913.8 & 2-Butanone & 5 & & U & & UG/L & $07 / 02 / 2009$ & & HCJ-166-09 \\
\hline GWA03601VL & USGS-103 & 913.8 & cis-1,2-Dichloroethylene & 1 & & U & & UG/L & $07 / 02 / 2009$ & & HCJ-166-09 \\
\hline GWA03601VL & USGS-103 & 913.8 & Chloroform & 1 & & $U$ & & UG/L & $07 / 02 / 2009$ & & HCJ-166-09 \\
\hline GWA03601VL & USGS-103 & 913.8 & 1,1,1-Trichloroethane & 1 & & $\mathrm{u}$ & & UG/L & $07 / 02 / 2009$ & & HCJ-166-09 \\
\hline GWA03601VL & USGS-103 & 913.8 & Cyclohexane & 1 & & U & & UG/L & $07 / 02 / 2009$ & & HCJ-166-09 \\
\hline GWA03601VL & USGS-103 & 913.8 & Carbon tetrachloride & 1 & & $U$ & & UG/L & $07 / 02 / 2009$ & & HCJ-166-09 \\
\hline GWA03601VL & USGS-103 & 913.8 & 1,2-Dichloroethane & 1 & & $\mathrm{u}$ & & UG/L & $07 / 02 / 2009$ & & HCJ-166-09 \\
\hline GWA03601VL & USGS-103 & 913.8 & Benzene & 1 & & U & & UG/L & $07 / 02 / 2009$ & & HCJ-166-09 \\
\hline GWA03601VL & USGS-103 & 913.8 & Trichloroethylene & 1 & & U & & UG/L & $07 / 02 / 2009$ & & HCJ-166-09 \\
\hline GWA03601VL & USGS-103 & 913.8 & 1,2-Dichloropropane & 1 & & 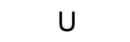 & & UG/L & $07 / 02 / 2009$ & & HCJ-166-09 \\
\hline GWA03601VL & USGS-103 & 913.8 & Methyl cyclohexane & 1 & & $\mathrm{U}$ & & UG/L & $07 / 02 / 2009$ & & HCJ-166-09 \\
\hline GWA03601VL & USGS-103 & 913.8 & Bromodichloromethane & 1 & & $\mathrm{U}$ & & UG/L & $07 / 02 / 2009$ & & HCJ-166-09 \\
\hline GWA03601VL & USGS-103 & 913.8 & 4-Methyl-2-pentanone & 5 & & $\mathrm{U}$ & & UG/L & $07 / 02 / 2009$ & & HCJ-166-09 \\
\hline GWA03601VL & USGS-103 & 913.8 & cis-1,3-Dichloropropylene & 1 & & $\mathrm{U}$ & & UG/L & $07 / 02 / 2009$ & & HCJ-166-09 \\
\hline GWA03601VL & USGS-103 & 913.8 & Toluene & 1 & & 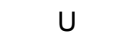 & & UG/L & $07 / 02 / 2009$ & & HCJ-166-09 \\
\hline GWA03601VL & USGS-103 & 913.8 & trans-1,3-Dichloropropylene & 1 & & $\mathrm{U}$ & & UG/L & $07 / 02 / 2009$ & & HCJ-166-09 \\
\hline
\end{tabular}


Table A-2. (continued).

\begin{tabular}{|c|c|c|c|c|c|c|c|c|c|c|c|}
\hline $\begin{array}{c}\text { Field Sample } \\
\text { Number }\end{array}$ & Location & Depth & Compound & $\begin{array}{c}\text { Sample } \\
\text { Result }\end{array}$ & $\begin{array}{c}\text { Sample } \\
\text { Error }\end{array}$ & $\begin{array}{c}\text { Result } \\
\text { Qualifier }\end{array}$ & $\begin{array}{c}\text { Validation } \\
\text { Flag }\end{array}$ & $\begin{array}{c}\text { Sample } \\
\text { Units }\end{array}$ & $\begin{array}{c}\text { Date Sample } \\
\text { Collected }\end{array}$ & MDA & L\&V Report Number \\
\hline GWA03601VL & USGS-103 & 913.8 & 1,1,2-Trichloroethane & 1 & & $\bar{U}$ & & UG/L & $07 / 02 / 2009$ & & HCJ-166-09 \\
\hline GWA03601VL & USGS-103 & 913.8 & 2-Hexanone & 5 & & $\mathrm{U}$ & & UG/L & 07/02/2009 & & HCJ-166-09 \\
\hline GWA03601VL & USGS-103 & 913.8 & Tetrachloroethylene & 1 & & BJ & $\mathrm{U}$ & UG/L & 07/02/2009 & & HCJ-166-09 \\
\hline GWA03601VL & USGS-103 & 913.8 & Dibromochloromethane & 1 & & $\mathrm{U}$ & & UG/L & 07/02/2009 & & HCJ-166-09 \\
\hline GWA03601VL & USGS-103 & 913.8 & 1,2-Dibromoethane & 1 & & $\mathrm{U}$ & & UG/L & 07/02/2009 & & HCJ-166-09 \\
\hline GWA03601VL & USGS-103 & 913.8 & Chlorobenzene & 1 & & $\mathrm{U}$ & & UG/L & 07/02/2009 & & HCJ-166-09 \\
\hline GWA03601VL & USGS-103 & 913.8 & Ethylbenzene & 1 & & $\mathrm{U}$ & & UG/L & 07/02/2009 & & HCJ-166-09 \\
\hline GWA03601VL & USGS-103 & 913.8 & Styrene & 1 & & U & & UG/L & 07/02/2009 & & HCJ-166-09 \\
\hline GWA03601VL & USGS-103 & 913.8 & Bromoform & 1 & & $\mathrm{U}$ & & UG/L & 07/02/2009 & & HCJ-166-09 \\
\hline GWA03601VL & USGS-103 & 913.8 & 1,1,2,2-Tetrachloroethane & 1 & & $\mathrm{U}$ & & UG/L & 07/02/2009 & & HCJ-166-09 \\
\hline GWA03601VL & USGS-103 & 913.8 & Isopropylbenzene & 1 & & $\mathrm{U}$ & & UG/L & 07/02/2009 & & HCJ-166-09 \\
\hline GWA03601VL & USGS-103 & 913.8 & 1,3-Dichlorobenzene & 1 & & $\mathrm{U}$ & & UG/L & 07/02/2009 & & HCJ-166-09 \\
\hline GWA03601VL & USGS-103 & 913.8 & 1,4-Dichlorobenzene & 1 & & $\mathrm{U}$ & & UG/L & 07/02/2009 & & HCJ-166-09 \\
\hline GWA03601VL & USGS-103 & 913.8 & 1,2-Dibromo-3-chloropropane & 1 & & U & & UG/L & 07/02/2009 & & HCJ-166-09 \\
\hline GWA03601VL & USGS-103 & 913.8 & Trichlorotrifluoroethane & 5 & & $\mathrm{U}$ & & UG/L & 07/02/2009 & & HCJ-166-09 \\
\hline GWA03601VL & USGS-103 & 913.8 & Xylene (Total) & 1 & & $\mathrm{U}$ & & UG/L & 07/02/2009 & & HCJ-166-09 \\
\hline GWA03601VL & USGS-103 & 913.8 & 1,2,4-Trichlorobenzene & 1 & & $\mathrm{U}$ & & UG/L & 07/02/2009 & & HCJ-166-09 \\
\hline GWA03601VL & USGS-103 & 913.8 & 1,2-Dichlorobenzene & 1 & & $\mathrm{U}$ & & UG/L & 07/02/2009 & & HCJ-166-09 \\
\hline GWA03601VL & USGS-103 & 913.8 & Unknown & 30.1 & & $\mathrm{~J}$ & & UG/L & 07/02/2009 & & \\
\hline GWA03701VL & USGS-103 & 999.4 & Dichlorodifluoromethane & 1 & & $\mathrm{U}$ & & UG/L & 07/07/2009 & & HCJ-166-09 \\
\hline GWA03701VL & USGS-103 & 999.4 & Chloromethane & 1 & & $\mathrm{U}$ & & UG/L & 07/07/2009 & & HCJ-166-09 \\
\hline GWA03701VL & USGS-103 & 999.4 & Vinyl Chloride & 1 & & $\mathrm{U}$ & & UG/L & 07/07/2009 & & HCJ-166-09 \\
\hline GWA03701VL & USGS-103 & 999.4 & Bromomethane & 1 & & $\mathrm{U}$ & & UG/L & 07/07/2009 & & HCJ-166-09 \\
\hline GWA03701VL & USGS-103 & 999.4 & Chloroethane & 1 & & $\mathrm{U}$ & & UG/L & 07/07/2009 & & HCJ-166-09 \\
\hline GWA03701VL & USGS-103 & 999.4 & Trichlorofluoromethane & 1 & & $\mathrm{U}$ & & UG/L & 07/07/2009 & & HCJ-166-09 \\
\hline GWA03701VL & USGS-103 & 999.4 & Acetone & 5 & & $\mathrm{U}$ & & UG/L & 07/07/2009 & & HCJ-166-09 \\
\hline GWA03701VL & USGS-103 & 999.4 & 1,1-Dichloroethene & 1 & & $\mathrm{U}$ & & UG/L & 07/07/2009 & & HCJ-166-09 \\
\hline GWA03701VL & USGS-103 & 999.4 & Methyl acetate & 5 & & $\mathrm{U}$ & & UG/L & 07/07/2009 & & HCJ-166-09 \\
\hline GWA03701VL & USGS-103 & 999.4 & Methylene Chloride & 5 & & $\mathrm{U}$ & & UG/L & 07/07/2009 & & HCJ-166-09 \\
\hline GWA03701VL & USGS-103 & 999.4 & Carbon disulfide & 5 & & $\mathrm{U}$ & & UG/L & 07/07/2009 & & HCJ-166-09 \\
\hline GWA03701VL & USGS-103 & 999.4 & Tert-butyl methyl ether & 1 & & $\mathrm{U}$ & & UG/L & 07/07/2009 & & HCJ-166-09 \\
\hline GWA03701VL & USGS-103 & 999.4 & trans-1,2-Dichloroethylene & 1 & & $\mathrm{U}$ & & UG/L & 07/07/2009 & & HCJ-166-09 \\
\hline GWA03701VL & USGS-103 & 999.4 & 1,1-Dichloroethane & 1 & & $\mathrm{U}$ & & UG/L & 07/07/2009 & & HCJ-166-09 \\
\hline GWA03701VL & USGS-103 & 999.4 & 2-Butanone & 5 & & $\mathrm{U}$ & & UG/L & 07/07/2009 & & HCJ-166-09 \\
\hline GWA03701VL & USGS-103 & 999.4 & cis-1,2-Dichloroethylene & 1 & & $\mathrm{U}$ & & UG/L & 07/07/2009 & & HCJ-166-09 \\
\hline GWA03701VL & USGS-103 & 999.4 & Chloroform & 1 & & $\mathrm{U}$ & & UG/L & 07/07/2009 & & HCJ-166-09 \\
\hline GWA03701VL & USGS-103 & 999.4 & 1,1,1-Trichloroethane & 1 & & $\mathrm{U}$ & & UG/L & 07/07/2009 & & HCJ-166-09 \\
\hline GWA03701VL & USGS-103 & 999.4 & Cyclohexane & 1 & & $U$ & & UG/L & 07/07/2009 & & HCJ-166-09 \\
\hline GWA03701VL & USGS-103 & 999.4 & Carbon tetrachloride & 1 & & $\mathrm{U}$ & & UG/L & 07/07/2009 & & HCJ-166-09 \\
\hline GWA03701VL & USGS-103 & 999.4 & 1,2-Dichloroethane & 1 & & $\mathrm{U}$ & & UG/L & 07/07/2009 & & HCJ-166-09 \\
\hline GWA03701VL & USGS-103 & 999.4 & Benzene & 1 & & $\mathrm{U}$ & & UG/L & 07/07/2009 & & HCJ-166-09 \\
\hline GWA03701VL & USGS-103 & 999.4 & Trichloroethylene & 1 & & $\mathrm{U}$ & & UG/L & 07/07/2009 & & HCJ-166-09 \\
\hline GWA03701VL & USGS-103 & 999.4 & 1,2-Dichloropropane & 1 & & $U$ & & UG/L & 07/07/2009 & & HCJ-166-09 \\
\hline GWA03701VL & USGS-103 & 999.4 & Methyl cyclohexane & 1 & & $\mathrm{U}$ & & UG/L & 07/07/2009 & & HCJ-166-09 \\
\hline
\end{tabular}


Table A-2. (continued).

\begin{tabular}{|c|c|c|c|c|c|c|c|c|c|c|c|}
\hline $\begin{array}{c}\text { Field Sample } \\
\text { Number }\end{array}$ & Location & Depth & Compound & $\begin{array}{c}\text { Sample } \\
\text { Result }\end{array}$ & $\begin{array}{c}\text { Sample } \\
\text { Error }\end{array}$ & $\begin{array}{c}\text { Result } \\
\text { Qualifier }\end{array}$ & $\begin{array}{c}\text { Validation } \\
\text { Flag }\end{array}$ & $\begin{array}{c}\text { Sample } \\
\text { Units }\end{array}$ & $\begin{array}{c}\text { Date Sample } \\
\text { Collected }\end{array}$ & MDA & L\&V Report Number \\
\hline GWA03701VL & USGS-103 & 999.4 & Bromodichloromethane & 1 & & $\bar{U}$ & & UG/L & $07 / 07 / 2009$ & & HCJ-166-09 \\
\hline GWA03701VL & USGS-103 & 999.4 & 4-Methyl-2-pentanone & 5 & & $\mathrm{U}$ & & UG/L & 07/07/2009 & & HCJ-166-09 \\
\hline GWA03701VL & USGS-103 & 999.4 & cis-1,3-Dichloropropylene & 1 & & $\mathrm{U}$ & & UG/L & 07/07/2009 & & HCJ-166-09 \\
\hline GWA03701VL & USGS-103 & 999.4 & Toluene & 1 & & $\mathrm{U}$ & & UG/L & 07/07/2009 & & HCJ-166-09 \\
\hline GWA03701VL & USGS-103 & 999.4 & trans-1,3-Dichloropropylene & 1 & & $\mathrm{U}$ & & UG/L & 07/07/2009 & & HCJ-166-09 \\
\hline GWA03701VL & USGS-103 & 999.4 & 1,1,2-Trichloroethane & 1 & & $\mathrm{U}$ & & UG/L & 07/07/2009 & & HCJ-166-09 \\
\hline GWA03701VL & USGS-103 & 999.4 & 2-Hexanone & 5 & & $\mathrm{U}$ & & UG/L & 07/07/2009 & & HCJ-166-09 \\
\hline GWA03701VL & USGS-103 & 999.4 & Tetrachloroethylene & 1 & & BJ & $\mathrm{U}$ & UG/L & 07/07/2009 & & HCJ-166-09 \\
\hline GWA03701VL & USGS-103 & 999.4 & Dibromochloromethane & 1 & & $\mathrm{U}$ & & UG/L & 07/07/2009 & & HCJ-166-09 \\
\hline GWA03701VL & USGS-103 & 999.4 & 1,2-Dibromoethane & 1 & & $\mathrm{U}$ & & UG/L & 07/07/2009 & & HCJ-166-09 \\
\hline GWA03701VL & USGS-103 & 999.4 & Chlorobenzene & 1 & & $\mathrm{U}$ & & UG/L & 07/07/2009 & & HCJ-166-09 \\
\hline GWA03701VL & USGS-103 & 999.4 & Ethylbenzene & 1 & & $\mathrm{U}$ & & UG/L & 07/07/2009 & & HCJ-166-09 \\
\hline GWA03701VL & USGS-103 & 999.4 & Styrene & 1 & & $\mathrm{U}$ & & UG/L & 07/07/2009 & & HCJ-166-09 \\
\hline GWA03701VL & USGS-103 & 999.4 & Bromoform & 1 & & $\mathrm{U}$ & & UG/L & 07/07/2009 & & HCJ-166-09 \\
\hline GWA03701VL & USGS-103 & 999.4 & 1,1,2,2-Tetrachloroethane & 1 & & $\mathrm{U}$ & & UG/L & 07/07/2009 & & HCJ-166-09 \\
\hline GWA03701VL & USGS-103 & 999.4 & Isopropylbenzene & 1 & & $\mathrm{U}$ & & UG/L & 07/07/2009 & & HCJ-166-09 \\
\hline GWA03701VL & USGS-103 & 999.4 & 1,3-Dichlorobenzene & 1 & & $\mathrm{U}$ & & UG/L & 07/07/2009 & & HCJ-166-09 \\
\hline GWA03701VL & USGS-103 & 999.4 & 1,4-Dichlorobenzene & 1 & & U & & UG/L & 07/07/2009 & & HCJ-166-09 \\
\hline GWA03701VL & USGS-103 & 999.4 & 1,2-Dibromo-3-chloropropane & 1 & & $\mathrm{U}$ & & UG/L & 07/07/2009 & & HCJ-166-09 \\
\hline GWA03701VL & USGS-103 & 999.4 & Trichlorotrifluoroethane & 5 & & $\mathrm{U}$ & & UG/L & 07/07/2009 & & HCJ-166-09 \\
\hline GWA03701VL & USGS-103 & 999.4 & Xylene (Total) & 1 & & $\mathrm{U}$ & & UG/L & 07/07/2009 & & HCJ-166-09 \\
\hline GWA03701VL & USGS-103 & 999.4 & 1,2,4-Trichlorobenzene & 1 & & U & & UG/L & 07/07/2009 & & HCJ-166-09 \\
\hline GWA03701VL & USGS-103 & 999.4 & 1,2-Dichlorobenzene & 1 & & $\mathrm{U}$ & & UG/L & 07/07/2009 & & HCJ-166-09 \\
\hline GWA03701VL & USGS-103 & 999.4 & Unknown & 21.1 & & $\mathrm{~J}$ & & UG/L & 07/07/2009 & & \\
\hline GWA03701VL & USGS-103 & 999.4 & Unknown & 9.65 & & $\mathrm{~J}$ & & UG/L & 07/07/2009 & & \\
\hline GWA03801VL & USGS-103 & 1095.1 & Dichlorodifluoromethane & 1 & & $\mathrm{U}$ & & UG/L & 07/08/2009 & & HCJ-166-09 \\
\hline GWA03801VL & USGS-103 & 1095.1 & Chloromethane & 1 & & $\mathrm{U}$ & & UG/L & 07/08/2009 & & HCJ-166-09 \\
\hline GWA03801VL & USGS-103 & 1095.1 & Vinyl Chloride & 1 & & $\mathrm{U}$ & & UG/L & 07/08/2009 & & HCJ-166-09 \\
\hline GWA03801VL & USGS-103 & 1095.1 & Bromomethane & 1 & & $\mathrm{U}$ & & UG/L & 07/08/2009 & & HCJ-166-09 \\
\hline GWA03801VL & USGS-103 & 1095.1 & Chloroethane & 1 & & $\mathrm{U}$ & & UG/L & 07/08/2009 & & HCJ-166-09 \\
\hline GWA03801VL & USGS-103 & 1095.1 & Trichlorofluoromethane & 1 & & $\mathrm{U}$ & & UG/L & 07/08/2009 & & HCJ-166-09 \\
\hline GWA03801VL & USGS-103 & 1095.1 & Acetone & 5 & & $\mathrm{U}$ & & UG/L & 07/08/2009 & & HCJ-166-09 \\
\hline GWA03801VL & USGS-103 & 1095.1 & 1,1-Dichloroethene & 1 & & $\mathrm{U}$ & & UG/L & 07/08/2009 & & HCJ-166-09 \\
\hline GWA03801VL & USGS-103 & 1095.1 & Methyl acetate & 5 & & $\mathrm{U}$ & & UG/L & 07/08/2009 & & HCJ-166-09 \\
\hline GWA03801VL & USGS-103 & 1095.1 & Methylene Chloride & 5 & & $\mathrm{U}$ & & UG/L & 07/08/2009 & & HCJ-166-09 \\
\hline GWA03801VL & USGS-103 & 1095.1 & Carbon disulfide & 5 & & $\mathrm{U}$ & & UG/L & 07/08/2009 & & HCJ-166-09 \\
\hline GWA03801VL & USGS-103 & 1095.1 & Tert-butyl methyl ether & 1 & & $\mathrm{U}$ & & UG/L & 07/08/2009 & & HCJ-166-09 \\
\hline GWA03801VL & USGS-103 & 1095.1 & trans-1,2-Dichloroethylene & 1 & & $U$ & & UG/L & 07/08/2009 & & HCJ-166-09 \\
\hline GWA03801VL & USGS-103 & 1095.1 & 1,1-Dichloroethane & 1 & & $\mathrm{U}$ & & UG/L & 07/08/2009 & & HCJ-166-09 \\
\hline GWA03801VL & USGS-103 & 1095.1 & 2-Butanone & 5 & & $\mathrm{U}$ & & UG/L & 07/08/2009 & & HCJ-166-09 \\
\hline GWA03801VL & USGS-103 & 1095.1 & cis-1,2-Dichloroethylene & 1 & & $\mathrm{U}$ & & UG/L & 07/08/2009 & & HCJ-166-09 \\
\hline GWA03801VL & USGS-103 & 1095.1 & Chloroform & 1 & & $\mathrm{U}$ & & UG/L & 07/08/2009 & & HCJ-166-09 \\
\hline GWA03801VL & USGS-103 & 1095.1 & 1,1,1-Trichloroethane & 1 & & $\mathrm{U}$ & & UG/L & 07/08/2009 & & HCJ-166-09 \\
\hline GWA03801VL & USGS-103 & 1095.1 & Cyclohexane & 1 & & $\mathrm{U}$ & & UG/L & 07/08/2009 & & HCJ-166-09 \\
\hline
\end{tabular}


Table A-2. (continued).

\begin{tabular}{|c|c|c|c|c|c|c|c|c|c|c|c|}
\hline $\begin{array}{c}\text { Field Sample } \\
\text { Number }\end{array}$ & Location & Depth & Compound & $\begin{array}{c}\text { Sample } \\
\text { Result }\end{array}$ & $\begin{array}{c}\text { Sample } \\
\text { Error }\end{array}$ & $\begin{array}{c}\text { Result } \\
\text { Qualifier } \\
\end{array}$ & $\begin{array}{c}\text { Validation } \\
\text { Flag }\end{array}$ & $\begin{array}{c}\text { Sample } \\
\text { Units }\end{array}$ & $\begin{array}{c}\text { Date Sample } \\
\text { Collected }\end{array}$ & MDA & L\&V Report Number \\
\hline GWA03801VL & USGS-103 & 1095.1 & Carbon tetrachloride & 1 & & $U$ & & UG/L & $07 / 08 / 2009$ & & HCJ-166-09 \\
\hline GWA03801VL & USGS-103 & 1095.1 & 1,2-Dichloroethane & 1 & & U & & UG/L & $07 / 08 / 2009$ & & HCJ-166-09 \\
\hline GWA03801VL & USGS-103 & 1095.1 & Benzene & 1 & & $\mathrm{u}$ & & UG/L & 07/08/2009 & & HCJ-166-09 \\
\hline GWA03801VL & USGS-103 & 1095.1 & Trichloroethylene & 1 & & $\mathrm{U}$ & & UG/L & 07/08/2009 & & HCJ-166-09 \\
\hline GWA03801VL & USGS-103 & 1095.1 & 1,2-Dichloropropane & 1 & & U & & UG/L & $07 / 08 / 2009$ & & HCJ-166-09 \\
\hline GWA03801VL & USGS-103 & 1095.1 & Methyl cyclohexane & 1 & & $\mathrm{U}$ & & UG/L & $07 / 08 / 2009$ & & HCJ-166-09 \\
\hline GWA03801VL & USGS-103 & 1095.1 & Bromodichloromethane & 1 & & $\mathrm{U}$ & & UG/L & 07/08/2009 & & HCJ-166-09 \\
\hline GWA03801VL & USGS-103 & 1095.1 & 4-Methyl-2-pentanone & 5 & & U & & UG/L & $07 / 08 / 2009$ & & HCJ-166-09 \\
\hline GWA03801VL & USGS-103 & 1095.1 & cis-1,3-Dichloropropylene & 1 & & U & & UG/L & $07 / 08 / 2009$ & & HCJ-166-09 \\
\hline GWA03801VL & USGS-103 & 1095.1 & Toluene & 1 & & $\mathrm{U}$ & & UG/L & 07/08/2009 & & HCJ-166-09 \\
\hline GWA03801VL & USGS-103 & 1095.1 & trans-1,3-Dichloropropylene & 1 & & $\mathrm{U}$ & & UG/L & $07 / 08 / 2009$ & & HCJ-166-09 \\
\hline GWA03801VL & USGS-103 & 1095.1 & 1,1,2-Trichloroethane & 1 & & U & & UG/L & $07 / 08 / 2009$ & & HCJ-166-09 \\
\hline GWA03801VL & USGS-103 & 1095.1 & 2-Hexanone & 5 & & U & & UG/L & $07 / 08 / 2009$ & & HCJ-166-09 \\
\hline GWA03801VL & USGS-103 & 1095.1 & Tetrachloroethylene & 1 & & BJ & $\mathrm{u}$ & UG/L & $07 / 08 / 2009$ & & HCJ-166-09 \\
\hline GWA03801VL & USGS-103 & 1095.1 & Dibromochloromethane & 1 & & $\mathrm{U}$ & & UG/L & $07 / 08 / 2009$ & & HCJ-166-09 \\
\hline GWA03801VL & USGS-103 & 1095.1 & 1,2-Dibromoethane & 1 & & U & & UG/L & $07 / 08 / 2009$ & & HCJ-166-09 \\
\hline GWA03801VL & USGS-103 & 1095.1 & Chlorobenzene & 1 & & $\mathrm{u}$ & & UG/L & $07 / 08 / 2009$ & & HCJ-166-09 \\
\hline GWA03801VL & USGS-103 & 1095.1 & Ethylbenzene & 1 & & $\mathrm{u}$ & & UG/L & 07/08/2009 & & HCJ-166-09 \\
\hline GWA03801VL & USGS-103 & 1095.1 & Styrene & 1 & & U & & UG/L & $07 / 08 / 2009$ & & HCJ-166-09 \\
\hline GWA03801VL & USGS-103 & 1095.1 & Bromoform & 1 & & $u$ & & UG/L & $07 / 08 / 2009$ & & HCJ-166-09 \\
\hline GWA03801VL & USGS-103 & 1095.1 & 1,1,2,2-Tetrachloroethane & 1 & & $\mathrm{u}$ & & UG/L & 07/08/2009 & & HCJ-166-09 \\
\hline GWA03801VL & USGS-103 & 1095.1 & Isopropylbenzene & 1 & & U & & UG/L & 07/08/2009 & & HCJ-166-09 \\
\hline GWA03801VL & USGS-103 & 1095.1 & 1,3-Dichlorobenzene & 1 & & U & & UG/L & $07 / 08 / 2009$ & & HCJ-166-09 \\
\hline GWA03801VL & USGS-103 & 1095.1 & 1,4-Dichlorobenzene & 1 & & U & & UG/L & $07 / 08 / 2009$ & & HCJ-166-09 \\
\hline GWA03801VL & USGS-103 & 1095.1 & 1,2-Dibromo-3-chloropropane & 1 & & $\mathrm{u}$ & & UG/L & $07 / 08 / 2009$ & & HCJ-166-09 \\
\hline GWA03801VL & USGS-103 & 1095.1 & Trichlorotrifluoroethane & 5 & & U & & UG/L & $07 / 08 / 2009$ & & HCJ-166-09 \\
\hline GWA03801VL & USGS-103 & 1095.1 & Xylene (Total) & 1 & & $\mathrm{U}$ & & UG/L & 07/08/2009 & & HCJ-166-09 \\
\hline GWA03801VL & USGS-103 & 1095.1 & 1,2,4-Trichlorobenzene & 1 & & $\mathrm{u}$ & & UG/L & $07 / 08 / 2009$ & & HCJ-166-09 \\
\hline GWA03801VL & USGS-103 & 1095.1 & 1,2-Dichlorobenzene & 1 & & U & & UG/L & 07/08/2009 & & HCJ-166-09 \\
\hline GWA03801VL & USGS-103 & 1095.1 & Unknown & 29.9 & & $\mathrm{~J}$ & & UG/L & $07 / 08 / 2009$ & & \\
\hline GWA03901VL & USGS-103 & 1220.2 & Dichlorodifluoromethane & 1 & & $u$ & & UG/L & 07/09/2009 & & HCJ-166-09 \\
\hline GWA03901VL & USGS-103 & 1220.2 & Chloromethane & 1 & & $\mathrm{u}$ & & UG/L & 07/09/2009 & & HCJ-166-09 \\
\hline GWA03901VL & USGS-103 & 1220.2 & Vinyl Chloride & 1 & & U & & UG/L & $07 / 09 / 2009$ & & HCJ-166-09 \\
\hline GWA03901VL & USGS-103 & 1220.2 & Bromomethane & 1 & & $u$ & & UG/L & $07 / 09 / 2009$ & & HCJ-166-09 \\
\hline GWA03901VL & USGS-103 & 1220.2 & Chloroethane & 1 & & $\mathrm{u}$ & & UG/L & 07/09/2009 & & HCJ-166-09 \\
\hline GWA03901VL & USGS-103 & 1220.2 & Trichlorofluoromethane & 1 & & $\mathrm{U}$ & & UG/L & 07/09/2009 & & HCJ-166-09 \\
\hline GWA03901VL & USGS-103 & 1220.2 & Acetone & 5 & & U & & UG/L & 07/09/2009 & & HCJ-166-09 \\
\hline GWA03901VL & USGS-103 & 1220.2 & 1,1-Dichloroethene & 1 & & $u$ & & UG/L & $07 / 09 / 2009$ & & HCJ-166-09 \\
\hline GWA03901VL & USGS-103 & 1220.2 & Methyl acetate & 5 & & $\mathrm{U}$ & & UG/L & $07 / 09 / 2009$ & & HCJ-166-09 \\
\hline GWA03901VL & USGS-103 & 1220.2 & Methylene Chloride & 5 & & $\mathrm{U}$ & & UG/L & 07/09/2009 & & HCJ-166-09 \\
\hline GWA03901VL & USGS-103 & 1220.2 & Carbon disulfide & 5 & & $\mathrm{U}$ & & UG/L & 07/09/2009 & & HCJ-166-09 \\
\hline GWA03901VL & USGS-103 & 1220.2 & Tert-butyl methyl ether & 1 & & $\mathrm{U}$ & & UG/L & 07/09/2009 & & HCJ-166-09 \\
\hline GWA03901VL & USGS-103 & 1220.2 & trans-1,2-Dichloroethylene & 1 & & $u$ & & UG/L & 07/09/2009 & & HCJ-166-09 \\
\hline GWA03901VL & USGS-103 & 1220.2 & 1,1-Dichloroethane & 1 & & U & & UG/L & $07 / 09 / 2009$ & & HCJ-166-09 \\
\hline
\end{tabular}


Table A-2. (continued).

\begin{tabular}{|c|c|c|c|c|c|c|c|c|c|c|c|}
\hline $\begin{array}{c}\text { Field Sample } \\
\text { Number }\end{array}$ & Location & Depth & Compound & $\begin{array}{c}\text { Sample } \\
\text { Result }\end{array}$ & $\begin{array}{c}\text { Sample } \\
\text { Error }\end{array}$ & $\begin{array}{c}\text { Result } \\
\text { Qualifier } \\
\end{array}$ & $\begin{array}{c}\text { Validation } \\
\text { Flag }\end{array}$ & $\begin{array}{c}\text { Sample } \\
\text { Units }\end{array}$ & $\begin{array}{c}\text { Date Sample } \\
\text { Collected }\end{array}$ & MDA & L\&V Report Number \\
\hline GWA03901VL & USGS-103 & 1220.2 & 2-Butanone & 5 & & $U$ & & UG/L & $07 / 09 / 2009$ & & HCJ-166-09 \\
\hline GWA03901VL & USGS-103 & 1220.2 & cis-1,2-Dichloroethylene & 1 & & U & & UG/L & $07 / 09 / 2009$ & & HCJ-166-09 \\
\hline GWA03901VL & USGS-103 & 1220.2 & Chloroform & 1 & & $\mathrm{U}$ & & UG/L & 07/09/2009 & & HCJ-166-09 \\
\hline GWA03901VL & USGS-103 & 1220.2 & 1,1,1-Trichloroethane & 1 & & $\mathrm{U}$ & & UG/L & 07/09/2009 & & HCJ-166-09 \\
\hline GWA03901VL & USGS-103 & 1220.2 & Cyclohexane & 1 & & U & & UG/L & 07/09/2009 & & HCJ-166-09 \\
\hline GWA03901VL & USGS-103 & 1220.2 & Carbon tetrachloride & 1 & & $\mathrm{U}$ & & UG/L & 07/09/2009 & & HCJ-166-09 \\
\hline GWA03901VL & USGS-103 & 1220.2 & 1,2-Dichloroethane & 1 & & $\mathrm{U}$ & & UG/L & 07/09/2009 & & HCJ-166-09 \\
\hline GWA03901VL & USGS-103 & 1220.2 & Benzene & 1 & & U & & UG/L & 07/09/2009 & & HCJ-166-09 \\
\hline GWA03901VL & USGS-103 & 1220.2 & Trichloroethylene & 1 & & U & & UG/L & $07 / 09 / 2009$ & & HCJ-166-09 \\
\hline GWA03901VL & USGS-103 & 1220.2 & 1,2-Dichloropropane & 1 & & $\mathrm{U}$ & & UG/L & 07/09/2009 & & HCJ-166-09 \\
\hline GWA03901VL & USGS-103 & 1220.2 & Methyl cyclohexane & 1 & & $\mathrm{U}$ & & UG/L & 07/09/2009 & & HCJ-166-09 \\
\hline GWA03901VL & USGS-103 & 1220.2 & Bromodichloromethane & 1 & & $U$ & & UG/L & $07 / 09 / 2009$ & & HCJ-166-09 \\
\hline GWA03901VL & USGS-103 & 1220.2 & 4-Methyl-2-pentanone & 5 & & U & & UG/L & $07 / 09 / 2009$ & & HCJ-166-09 \\
\hline GWA03901VL & USGS-103 & 1220.2 & cis-1,3-Dichloropropylene & 1 & & $\mathrm{u}$ & & UG/L & $07 / 09 / 2009$ & & HCJ-166-09 \\
\hline GWA03901VL & USGS-103 & 1220.2 & Toluene & 1 & & U & & UG/L & 07/09/2009 & & HCJ-166-09 \\
\hline GWA03901VL & USGS-103 & 1220.2 & trans-1,3-Dichloropropylene & 1 & & U & & UG/L & $07 / 09 / 2009$ & & HCJ-166-09 \\
\hline GWA03901VL & USGS-103 & 1220.2 & 1,1,2-Trichloroethane & 1 & & $\mathrm{u}$ & & UG/L & 07/09/2009 & & HCJ-166-09 \\
\hline GWA03901VL & USGS-103 & 1220.2 & 2-Hexanone & 5 & & $\mathrm{u}$ & & UG/L & 07/09/2009 & & HCJ-166-09 \\
\hline GWA03901VL & USGS-103 & 1220.2 & Tetrachloroethylene & 1 & & BJ & $\mathrm{U}$ & UG/L & 07/09/2009 & & HCJ-166-09 \\
\hline GWA03901VL & USGS-103 & 1220.2 & Dibromochloromethane & 1 & & $U$ & & UG/L & $07 / 09 / 2009$ & & HCJ-166-09 \\
\hline GWA03901VL & USGS-103 & 1220.2 & 1,2-Dibromoethane & 1 & & $\mathrm{u}$ & & UG/L & 07/09/2009 & & HCJ-166-09 \\
\hline GWA03901VL & USGS-103 & 1220.2 & Chlorobenzene & 1 & & U & & UG/L & 07/09/2009 & & HCJ-166-09 \\
\hline GWA03901VL & USGS-103 & 1220.2 & Ethylbenzene & 1 & & U & & UG/L & $07 / 09 / 2009$ & & HCJ-166-09 \\
\hline GWA03901VL & USGS-103 & 1220.2 & Styrene & 1 & & U & & UG/L & $07 / 09 / 2009$ & & HCJ-166-09 \\
\hline GWA03901VL & USGS-103 & 1220.2 & Bromoform & 1 & & $\mathrm{u}$ & & UG/L & 07/09/2009 & & HCJ-166-09 \\
\hline GWA03901VL & USGS-103 & 1220.2 & 1,1,2,2-Tetrachloroethane & 1 & & U & & UG/L & 07/09/2009 & & HCJ-166-09 \\
\hline GWA03901VL & USGS-103 & 1220.2 & Isopropylbenzene & 1 & & $\mathrm{U}$ & & UG/L & 07/09/2009 & & HCJ-166-09 \\
\hline GWA03901VL & USGS-103 & 1220.2 & 1,3-Dichlorobenzene & 1 & & $\mathrm{u}$ & & UG/L & 07/09/2009 & & HCJ-166-09 \\
\hline GWA03901VL & USGS-103 & 1220.2 & 1,4-Dichlorobenzene & 1 & & U & & UG/L & 07/09/2009 & & HCJ-166-09 \\
\hline GWA03901VL & USGS-103 & 1220.2 & 1,2-Dibromo-3-chloropropane & 1 & & U & & UG/L & 07/09/2009 & & HCJ-166-09 \\
\hline GWA03901VL & USGS-103 & 1220.2 & Trichlorotrifluoroethane & 5 & & $u$ & & UG/L & 07/09/2009 & & HCJ-166-09 \\
\hline GWA03901VL & USGS-103 & 1220.2 & Xylene (Total) & 1 & & $\mathrm{u}$ & & UG/L & 07/09/2009 & & HCJ-166-09 \\
\hline GWA03901VL & USGS-103 & 1220.2 & 1,2,4-Trichlorobenzene & 1 & & $\mathrm{U}$ & & UG/L & $07 / 09 / 2009$ & & HCJ-166-09 \\
\hline GWA03901VL & USGS-103 & 1220.2 & 1,2-Dichlorobenzene & 1 & & U & & UG/L & 07/09/2009 & & HCJ-166-09 \\
\hline GWA03901VL & USGS-103 & 1220.2 & Unknown & 29.6 & & $\mathrm{~J}$ & & UG/L & 07/09/2009 & & \\
\hline GWA04001VL & USGS-103 & 1269.4 & Dichlorodifluoromethane & 1 & & U & & UG/L & $07 / 13 / 2009$ & & HCJ-167-09 \\
\hline GWA04001VL & USGS-103 & 1269.4 & Chloromethane & 1 & & U & & UG/L & $07 / 13 / 2009$ & & HCJ-167-09 \\
\hline GWA04001VL & USGS-103 & 1269.4 & Vinyl Chloride & 1 & & $u$ & & UG/L & $07 / 13 / 2009$ & & HCJ-167-09 \\
\hline GWA04001VL & USGS-103 & 1269.4 & Bromomethane & 1 & & $\mathrm{U}$ & & UG/L & $07 / 13 / 2009$ & & HCJ-167-09 \\
\hline GWA04001VL & USGS-103 & 1269.4 & Chloroethane & 1 & & $\mathrm{U}$ & & UG/L & $07 / 13 / 2009$ & & HCJ-167-09 \\
\hline GWA04001VL & USGS-103 & 1269.4 & Trichlorofluoromethane & 1 & & $\mathrm{U}$ & & UG/L & $07 / 13 / 2009$ & & HCJ-167-09 \\
\hline GWA04001VL & USGS-103 & 1269.4 & Acetone & 5 & & $\mathrm{~J}$ & $\mathrm{U}$ & UG/L & $07 / 13 / 2009$ & & HCJ-167-09 \\
\hline GWA04001VL & USGS-103 & 1269.4 & 1,1-Dichloroethene & 1 & & 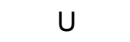 & & UG/L & $07 / 13 / 2009$ & & HCJ-167-09 \\
\hline GWA04001VL & USGS-103 & 1269.4 & Methyl acetate & 5 & & U & & UG/L & 07/13/2009 & & HCJ-167-09 \\
\hline
\end{tabular}


Table A-2. (continued).

\begin{tabular}{|c|c|c|c|c|c|c|c|c|c|c|c|}
\hline $\begin{array}{c}\text { Field Sample } \\
\text { Number }\end{array}$ & Location & Depth & Compound & $\begin{array}{l}\text { Sample } \\
\text { Result }\end{array}$ & $\begin{array}{l}\text { Sample } \\
\text { Error }\end{array}$ & $\begin{array}{c}\text { Result } \\
\text { Qualifier }\end{array}$ & $\begin{array}{c}\text { Validation } \\
\text { Flag }\end{array}$ & $\begin{array}{c}\text { Sample } \\
\text { Units }\end{array}$ & $\begin{array}{c}\text { Date Sample } \\
\text { Collected }\end{array}$ & MDA & L\&V Report Number \\
\hline GWA04001VL & USGS-103 & 1269.4 & Methylene Chloride & 5 & & $\bar{U}$ & & UG/L & $07 / 13 / 2009$ & & HCJ-167-09 \\
\hline GWA04001VL & USGS-103 & 1269.4 & Carbon disulfide & 5 & & $\mathrm{U}$ & & UG/L & 07/13/2009 & & HCJ-167-09 \\
\hline GWA04001VL & USGS-103 & 1269.4 & Tert-butyl methyl ether & 1 & & $\mathrm{U}$ & & UG/L & 07/13/2009 & & HCJ-167-09 \\
\hline GWA04001VL & USGS-103 & 1269.4 & trans-1,2-Dichloroethylene & 1 & & $\mathrm{U}$ & & UG/L & 07/13/2009 & & HCJ-167-09 \\
\hline GWA04001VL & USGS-103 & 1269.4 & 1,1-Dichloroethane & 1 & & $\mathrm{U}$ & & UG/L & 07/13/2009 & & HCJ-167-09 \\
\hline GWA04001VL & USGS-103 & 1269.4 & 2-Butanone & 5 & & $\mathrm{U}$ & & UG/L & 07/13/2009 & & HCJ-167-09 \\
\hline GWA04001VL & USGS-103 & 1269.4 & cis-1,2-Dichloroethylene & 1 & & U & & UG/L & 07/13/2009 & & HCJ-167-09 \\
\hline GWA04001VL & USGS-103 & 1269.4 & Chloroform & 1 & & U & & UG/L & 07/13/2009 & & HCJ-167-09 \\
\hline GWA04001VL & USGS-103 & 1269.4 & 1,1,1-Trichloroethane & 1 & & $\mathrm{U}$ & & UG/L & 07/13/2009 & & HCJ-167-09 \\
\hline GWA04001VL & USGS-103 & 1269.4 & Cyclohexane & 1 & & $\mathrm{U}$ & & UG/L & 07/13/2009 & & HCJ-167-09 \\
\hline GWA04001VL & USGS-103 & 1269.4 & Carbon tetrachloride & 1 & & $\mathrm{U}$ & & UG/L & 07/13/2009 & & HCJ-167-09 \\
\hline GWA04001VL & USGS-103 & 1269.4 & 1,2-Dichloroethane & 1 & & $\mathrm{U}$ & & UG/L & 07/13/2009 & & HCJ-167-09 \\
\hline GWA04001VL & USGS-103 & 1269.4 & Benzene & 1 & & $\mathrm{U}$ & & UG/L & 07/13/2009 & & HCJ-167-09 \\
\hline GWA04001VL & USGS-103 & 1269.4 & Trichloroethylene & 1 & & $\mathrm{U}$ & & UG/L & 07/13/2009 & & HCJ-167-09 \\
\hline GWA04001VL & USGS-103 & 1269.4 & 1,2-Dichloropropane & 1 & & $\mathrm{U}$ & & UG/L & 07/13/2009 & & HCJ-167-09 \\
\hline GWA04001VL & USGS-103 & 1269.4 & Methyl cyclohexane & 1 & & $\mathrm{U}$ & & UG/L & 07/13/2009 & & HCJ-167-09 \\
\hline GWA04001VL & USGS-103 & 1269.4 & Bromodichloromethane & 1 & & $\mathrm{U}$ & & UG/L & 07/13/2009 & & HCJ-167-09 \\
\hline GWA04001VL & USGS-103 & 1269.4 & 4-Methyl-2-pentanone & 5 & & $\mathrm{U}$ & & UG/L & 07/13/2009 & & HCJ-167-09 \\
\hline GWA04001VL & USGS-103 & 1269.4 & cis-1,3-Dichloropropylene & 1 & & $\mathrm{U}$ & & UG/L & 07/13/2009 & & HCJ-167-09 \\
\hline GWA04001VL & USGS-103 & 1269.4 & Toluene & 1 & & $\mathrm{U}$ & & UG/L & 07/13/2009 & & HCJ-167-09 \\
\hline GWA04001VL & USGS-103 & 1269.4 & trans-1,3-Dichloropropylene & 1 & & $\mathrm{U}$ & & UG/L & 07/13/2009 & & HCJ-167-09 \\
\hline GWA04001VL & USGS-103 & 1269.4 & 1,1,2-Trichloroethane & 1 & & $\mathrm{U}$ & & UG/L & 07/13/2009 & & HCJ-167-09 \\
\hline GWA04001VL & USGS-103 & 1269.4 & 2-Hexanone & 5 & & $\mathrm{U}$ & & UG/L & $07 / 13 / 2009$ & & HCJ-167-09 \\
\hline GWA04001VL & USGS-103 & 1269.4 & Tetrachloroethylene & 1 & & $\mathrm{U}$ & & UG/L & 07/13/2009 & & HCJ-167-09 \\
\hline GWA04001VL & USGS-103 & 1269.4 & Dibromochloromethane & 1 & & $\mathrm{U}$ & & UG/L & 07/13/2009 & & HCJ-167-09 \\
\hline GWA04001VL & USGS-103 & 1269.4 & 1,2-Dibromoethane & 1 & & $\mathrm{U}$ & & UG/L & 07/13/2009 & & HCJ-167-09 \\
\hline GWA04001VL & USGS-103 & 1269.4 & Chlorobenzene & 1 & & $\mathrm{U}$ & & UG/L & 07/13/2009 & & HCJ-167-09 \\
\hline GWA04001VL & USGS-103 & 1269.4 & Ethylbenzene & 1 & & $\mathrm{U}$ & & UG/L & 07/13/2009 & & HCJ-167-09 \\
\hline GWA04001VL & USGS-103 & 1269.4 & Styrene & 1 & & $\mathrm{U}$ & & UG/L & 07/13/2009 & & HCJ-167-09 \\
\hline GWA04001VL & USGS-103 & 1269.4 & Bromoform & 1 & & $\mathrm{U}$ & & UG/L & $07 / 13 / 2009$ & & HCJ-167-09 \\
\hline GWA04001VL & USGS-103 & 1269.4 & 1,1,2,2-Tetrachloroethane & 1 & & $\mathrm{U}$ & & UG/L & 07/13/2009 & & HCJ-167-09 \\
\hline GWA04001VL & USGS-103 & 1269.4 & Isopropylbenzene & 1 & & $\mathrm{U}$ & & UG/L & 07/13/2009 & & HCJ-167-09 \\
\hline GWA04001VL & USGS-103 & 1269.4 & 1,3-Dichlorobenzene & 1 & & $\mathrm{U}$ & & UG/L & $07 / 13 / 2009$ & & HCJ-167-09 \\
\hline GWA04001VL & USGS-103 & 1269.4 & 1,4-Dichlorobenzene & 1 & & $\mathrm{U}$ & & UG/L & 07/13/2009 & & HCJ-167-09 \\
\hline GWA04001VL & USGS-103 & 1269.4 & 1,2-Dibromo-3-chloropropane & 1 & & $\mathrm{U}$ & & UG/L & 07/13/2009 & & HCJ-167-09 \\
\hline GWA04001VL & USGS-103 & 1269.4 & Trichlorotrifluoroethane & 5 & & U & & UG/L & 07/13/2009 & & HCJ-167-09 \\
\hline GWA04001VL & USGS-103 & 1269.4 & Xylene (Total) & 1 & & $\mathrm{U}$ & & UG/L & 07/13/2009 & & HCJ-167-09 \\
\hline GWA04001VL & USGS-103 & 1269.4 & 1,2,4-Trichlorobenzene & 1 & & $U$ & & UG/L & 07/13/2009 & & HCJ-167-09 \\
\hline GWA04001VL & USGS-103 & 1269.4 & 1,2-Dichlorobenzene & 1 & & $\mathrm{U}$ & & UG/L & $07 / 13 / 2009$ & & HCJ-167-09 \\
\hline GWA04001VL & USGS-103 & 1269.4 & unknown & 22.3 & & $\mathrm{~J}$ & & UG/L & 07/13/2009 & & \\
\hline GWA03501R8 & USGS-103 & 805.2 & Tritium & $7.06 \mathrm{E}+01$ & $1.06 \mathrm{E}+02$ & & $U$ & $\mathrm{PCl} / \mathrm{L}$ & 07/01/2009 & $3.60 \mathrm{E}+02$ & BAM-116-09 \\
\hline GWA03601R8 & USGS-103 & 913.8 & Tritium & $1.22 \mathrm{E}+02$ & $8.70 \mathrm{E}+01$ & & $\mathrm{U}$ & $\mathrm{PCl} / \mathrm{L}$ & 07/02/2009 & $2.87 \mathrm{E}+02$ & BAM-116-09 \\
\hline GWA03701R8 & USGS-103 & 999.4 & Tritium & $1.07 \mathrm{E}+02$ & $8.64 \mathrm{E}+01$ & & $U$ & $\mathrm{PCl} / \mathrm{L}$ & 07/07/2009 & $2.87 \mathrm{E}+02$ & BAM-116-09 \\
\hline GWA03801R8 & USGS-103 & 1095.1 & Tritium & $3.60 \mathrm{E}+02$ & $9.99 \mathrm{E}+01$ & & & $\mathrm{PCI} / \mathrm{L}$ & 07/08/2009 & $2.86 \mathrm{E}+02$ & BAM-116-09 \\
\hline
\end{tabular}


Table A-2. (continued).

\begin{tabular}{|c|c|c|c|c|c|c|c|c|c|c|c|}
\hline $\begin{array}{c}\text { Field Sample } \\
\text { Number }\end{array}$ & Location & Depth & Compound & $\begin{array}{c}\text { Sample } \\
\text { Result }\end{array}$ & $\begin{array}{l}\text { Sample } \\
\text { Error }\end{array}$ & $\begin{array}{c}\text { Result } \\
\text { Qualifier }\end{array}$ & $\begin{array}{c}\text { Validation } \\
\text { Flag }\end{array}$ & $\begin{array}{c}\text { Sample } \\
\text { Units }\end{array}$ & $\begin{array}{c}\text { Date Sample } \\
\text { Collected }\end{array}$ & MDA & L\&V Report Number \\
\hline GWA03901R8 & USGS-103 & 1220.2 & Tritium & $2.50 \mathrm{E}+02$ & $9.34 \mathrm{E}+01$ & & UJ & $\mathrm{PCl} / \mathrm{L}$ & $07 / 09 / 2009$ & $2.86 \mathrm{E}+02$ & BAM-116-09 \\
\hline GWA04001R8 & USGS-103 & 1269.4 & Tritium & 4.37E+02 & $1.05 \mathrm{E}+02$ & & & $\mathrm{PCl} / \mathrm{L}$ & $07 / 13 / 2009$ & $2.86 \mathrm{E}+02$ & BAM-116-09 \\
\hline GWA03501RH & USGS-103 & 805.2 & Gross Alpha & $9.24 \mathrm{E}-01$ & 4.19E-01 & & UJ & $\mathrm{PCl} / \mathrm{L}$ & 07/01/2009 & $1.11 \mathrm{E}+00$ & BAM-115-09 \\
\hline GWA03501RH & USGS-103 & 805.2 & Gross Beta & $4.31 \mathrm{E}+00$ & $6.97 \mathrm{E}-01$ & & $\mathrm{~J}$ & $\mathrm{PCl} / \mathrm{L}$ & $07 / 01 / 2009$ & $1.24 \mathrm{E}+00$ & BAM-115-09 \\
\hline GWA03601RH & USGS-103 & 913.8 & Gross Alpha & $1.53 \mathrm{E}+00$ & $5.45 \mathrm{E}-01$ & & UJ & $\mathrm{PCl} / \mathrm{L}$ & $07 / 02 / 2009$ & $1.30 \mathrm{E}+00$ & BAM-115-09 \\
\hline GWA03601RH & USGS-103 & 913.8 & Gross Beta & $3.32 \mathrm{E}+00$ & 7.38E-01 & & $\mathrm{J}$ & $\mathrm{PCl} / \mathrm{L}$ & $07 / 02 / 2009$ & $1.88 \mathrm{E}+00$ & BAM-115-09 \\
\hline GWA03801RH & USGS-103 & 1095.1 & Gross Alpha & $1.88 \mathrm{E}+00$ & 5.71E-01 & & UJ & $\mathrm{PCl} / \mathrm{L}$ & $07 / 08 / 2009$ & $1.10 \mathrm{E}+00$ & BAM-115-09 \\
\hline GWA03801RH & USGS-103 & 1095.1 & Gross Beta & $2.73 \mathrm{E}+00$ & $5.96 \mathrm{E}-01$ & & UJ & $\mathrm{PCl} / \mathrm{L}$ & 07/08/2009 & $1.42 \mathrm{E}+00$ & BAM-115-09 \\
\hline GWA03701RH & USGS-103 & 999.4 & Gross Alpha & $1.07 \mathrm{E}+00$ & 4.49E-01 & & UJ & $\mathrm{PCl} / \mathrm{L}$ & 07/07/2009 & $1.07 \mathrm{E}+00$ & BAM-115-09 \\
\hline GWA03701RH & USGS-103 & 999.4 & Gross Beta & $3.32 \mathrm{E}+00$ & $5.94 \mathrm{E}-01$ & & $\mathrm{~J}$ & $\mathrm{PCl} / \mathrm{L}$ & 07/07/2009 & $1.11 \mathrm{E}+00$ & BAM-115-09 \\
\hline GWA03901RH & USGS-103 & 1220.2 & Gross Alpha & $1.38 \mathrm{E}-01$ & 3.23E-01 & & U & $\mathrm{PCl} / \mathrm{L}$ & $07 / 09 / 2009$ & $1.22 \mathrm{E}+00$ & BAM-115-09 \\
\hline GWA03901RH & USGS-103 & 1220.2 & Gross Beta & $2.00 \mathrm{E}+00$ & 3.94E-01 & & UJ & $\mathrm{PCl} / \mathrm{L}$ & 07/09/2009 & $9.09 \mathrm{E}-01$ & BAM-115-09 \\
\hline GWA04001RH & USGS-103 & 1269.4 & Gross Alpha & $1.87 \mathrm{E}+00$ & $6.20 \mathrm{E}-01$ & & UJ & $\mathrm{PCl} / \mathrm{L}$ & $07 / 13 / 2009$ & $1.42 \mathrm{E}+00$ & BAM-115-09 \\
\hline GWA04001RH & USGS-103 & 1269.4 & Gross Beta & $2.88 \mathrm{E}+00$ & 7.90E-01 & & UJ & $\mathrm{PCl} / \mathrm{L}$ & $07 / 13 / 2009$ & $2.23 E+00$ & BAM-115-09 \\
\hline GWA03501RH & USGS-103 & 805.2 & Strontium-90 & $-5.51 \mathrm{E}-02$ & $1.02 \mathrm{E}-01$ & & $U$ & $\mathrm{PCl} / \mathrm{L}$ & 07/01/2009 & $3.82 \mathrm{E}-01$ & BAM-115-09 \\
\hline GWA03601RH & USGS-103 & 913.8 & Strontium-90 & 7.11E-02 & 1.10E-01 & & $u$ & $\mathrm{PCl} / \mathrm{L}$ & $07 / 02 / 2009$ & 3.83E-01 & BAM-115-09 \\
\hline GWA03801RH & USGS-103 & 1095.1 & Strontium-90 & 7.69E-02 & 1.04E-01 & & $U$ & $\mathrm{PCl} / \mathrm{L}$ & 07/08/2009 & 3.61E-01 & BAM-115-09 \\
\hline GWA03701RH & USGS-103 & 999.4 & Strontium-90 & 1.74E-02 & 1.11E-01 & & U & $\mathrm{PCl} / \mathrm{L}$ & 07/07/2009 & $4.00 \mathrm{E}-01$ & BAM-115-09 \\
\hline GWA03901RH & USGS-103 & 1220.2 & Strontium-90 & 7.68E-02 & 1.12E-01 & & U & $\mathrm{PCl} / \mathrm{L}$ & 07/09/2009 & $3.90 \mathrm{E}-01$ & BAM-115-09 \\
\hline GWA04001RH & USGS-103 & 1269.4 & Strontium-90 & 2.27E-02 & $9.80 \mathrm{E}-02$ & & $u$ & $\mathrm{PCl} / \mathrm{L}$ & $07 / 13 / 2009$ & 3.54E-01 & BAM-115-09 \\
\hline GWA03501RH & USGS-103 & 805.2 & Technetium-99 & $-1.04 \mathrm{E}-01$ & $2.38 \mathrm{E}+00$ & & U & $\mathrm{PCl} / \mathrm{L}$ & 07/01/2009 & $8.10 \mathrm{E}+00$ & BAM-115-09 \\
\hline GWA03601RH & USGS-103 & 913.8 & Technetium-99 & $-7.76 \mathrm{E}-01$ & $2.35 E+00$ & & U & $\mathrm{PCl} / \mathrm{L}$ & $07 / 02 / 2009$ & $8.05 E+00$ & BAM-115-09 \\
\hline GWA03801RH & USGS-103 & 1095.1 & Technetium-99 & $2.81 \mathrm{E}+00$ & $2.48 \mathrm{E}+00$ & & U & $\mathrm{PCl} / \mathrm{L}$ & $07 / 08 / 2009$ & $8.25 \mathrm{E}+00$ & BAM-115-09 \\
\hline GWA03701RH & USGS-103 & 999.4 & Technetium-99 & $3.26 \mathrm{E}+00$ & $2.49 \mathrm{E}+00$ & & U & $\mathrm{PCl} / \mathrm{L}$ & 07/07/2009 & $8.24 \mathrm{E}+00$ & BAM-115-09 \\
\hline GWA03901RH & USGS-103 & 1220.2 & Technetium-99 & $1.81 \mathrm{E}+00$ & $2.40 \mathrm{E}+00$ & & U & $\mathrm{PCl} / \mathrm{L}$ & 07/09/2009 & $8.03 E+00$ & BAM-115-09 \\
\hline GWA04001RH & USGS-103 & 1269.4 & Technetium-99 & 2.62E-01 & $2.40 \mathrm{E}+00$ & & U & $\mathrm{PCl} / \mathrm{L}$ & $07 / 13 / 2009$ & $8.16 \mathrm{E}+00$ & BAM-115-09 \\
\hline GWA03501UX & USGS-103 & 805.2 & lodine-129 & $1.02 \mathrm{E}-02$ & 2.57E-02 & & $u$ & $\mathrm{PCl} / \mathrm{L}$ & 07/01/2009 & 9.09E-02 & BAM-117-09 \\
\hline GWA03601UX & USGS-103 & 913.8 & lodine-129 & $-5.12 \mathrm{E}-02$ & 2.82E-02 & & $\mathrm{U}$ & $\mathrm{PCl} / \mathrm{L}$ & $07 / 02 / 2009$ & $8.88 \mathrm{E}-02$ & BAM-117-09 \\
\hline GWA03701UX & USGS-103 & 999.4 & lodine-129 & $1.94 \mathrm{E}-02$ & 2.39E-02 & & U & $\mathrm{PCl} / \mathrm{L}$ & $07 / 07 / 2009$ & $8.58 \mathrm{E}-02$ & BAM-117-09 \\
\hline GWA03801UX & USGS-103 & 1095.1 & lodine-129 & $-2.12 \mathrm{E}-02$ & 2.46E-02 & & U & $\mathrm{PCl} / \mathrm{L}$ & $07 / 08 / 2009$ & 7.49E-02 & BAM-117-09 \\
\hline GWA03901UX & USGS-103 & 1220.2 & lodine-129 & 3.13E-03 & 3.17E-02 & & 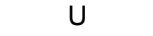 & $\mathrm{PCl} / \mathrm{L}$ & 07/09/2009 & 1.07E-01 & BAM-117-09 \\
\hline GWA04001UX & USGS-103 & 1269.4 & lodine-129 & $-1.04 \mathrm{E}-02$ & $1.08 \mathrm{E}-02$ & & $\mathrm{U}$ & $\mathrm{PCl} / \mathrm{L}$ & $07 / 13 / 2009$ & $3.45 \mathrm{E}-02$ & BAM-117-09 \\
\hline GWA03401R8 & USGS-103 & 681.9 & Tritium & $-2.80 \mathrm{E}+01$ & $1.06 \mathrm{E}+02$ & & U & $\mathrm{PCl} / \mathrm{L}$ & 07/01/2009 & $3.86 \mathrm{E}+02$ & BAM-119-09 \\
\hline GWA03401RH & USGS-103 & 681.9 & Gross Alpha & $3.84 \mathrm{E}+00$ & $9.53 \mathrm{E}-01$ & & & $\mathrm{PCl} / \mathrm{L}$ & 07/01/2009 & $1.22 \mathrm{E}+00$ & BAM-120-09 \\
\hline GWA03401RH & USGS-103 & 681.9 & Gross Beta & $2.62 \mathrm{E}+00$ & $6.71 \mathrm{E}-01$ & & & $\mathrm{PCl} / \mathrm{L}$ & 07/01/2009 & $1.56 \mathrm{E}+00$ & BAM-120-09 \\
\hline GWA03401RH & USGS-103 & 681.9 & Strontium-90 & 7.73E-02 & $1.04 \mathrm{E}-01$ & & U & $\mathrm{PCl} / \mathrm{L}$ & 07/01/2009 & $3.66 \mathrm{E}-01$ & BAM-120-09 \\
\hline GWA03401RH & USGS-103 & 681.9 & Technetium-99 & $-5.68 \mathrm{E}+00$ & $2.84 \mathrm{E}+00$ & & U & $\mathrm{PCl} / \mathrm{L}$ & 07/01/2009 & $9.84 \mathrm{E}+00$ & BAM-120-09 \\
\hline GWA03401UX & USGS-103 & 681.9 & lodine-129 & $-2.76 \mathrm{E}-03$ & 2.33E-02 & & $u$ & $\mathrm{PCl} / \mathrm{L}$ & 07/01/2009 & 7.74E-02 & BAM-118-09 \\
\hline GWA04701A1 & USGS-104 & 555 & Alkalinity, Total as $\mathrm{CaCO} 3$ & 122 & & & & MG/L & $06 / 15 / 2009$ & & HCJ-156-09 \\
\hline GWA04702A1 & USGS-104 & 555 & Alkalinity, Total as $\mathrm{CaCO} 3$ & 121 & & & & MG/L & $06 / 15 / 2009$ & & HCJ-156-09 \\
\hline GWA04701AN & USGS-104 & 555 & Bromide & 0 & & $U$ & & MG/L & $06 / 15 / 2009$ & & HCJ-158-09 \\
\hline GWA04701AN & USGS-104 & 555 & Chloride & 13.1 & & & & MG/L & $06 / 15 / 2009$ & & HCJ-158-09 \\
\hline GWA04701AN & USGS-104 & 555 & Fluoride & 0.231 & & $\mathrm{~J}$ & & MG/L & $06 / 15 / 2009$ & & HCJ-158-09 \\
\hline GWA04701AN & USGS-104 & 555 & Sulfate & 20.6 & & & & MG/L & $06 / 15 / 2009$ & & HCJ-158-09 \\
\hline
\end{tabular}


Table A-2. (continued).

\begin{tabular}{|c|c|c|c|c|c|c|c|c|c|c|c|}
\hline $\begin{array}{c}\text { Field Sample } \\
\text { Number }\end{array}$ & Location & Depth & Compound & $\begin{array}{c}\text { Sample } \\
\text { Result }\end{array}$ & $\begin{array}{l}\text { Sample } \\
\text { Error }\end{array}$ & $\begin{array}{c}\text { Result } \\
\text { Qualifier }\end{array}$ & $\begin{array}{l}\text { Validation } \\
\text { Flag }\end{array}$ & $\begin{array}{c}\text { Sample } \\
\text { Units }\end{array}$ & $\begin{array}{c}\text { Date Sample } \\
\text { Collected }\end{array}$ & MDA & L\&V Report Number \\
\hline GWA04702AN & USGS-104 & 555 & Bromide & 0 & & $\bar{U}$ & & MG/L & $06 / 15 / 2009$ & & HCJ-158-09 \\
\hline GWA04702AN & USGS-104 & 555 & Chloride & 13 & & & & MG/L & 06/15/2009 & & HCJ-158-09 \\
\hline GWA04702AN & USGS-104 & 555 & Fluoride & 0.23 & & J & & MG/L & 06/15/2009 & & HCJ-158-09 \\
\hline GWA04702AN & USGS-104 & 555 & Sulfate & 20.6 & & & & MG/L & 06/15/2009 & & HCJ-158-09 \\
\hline GWA04701N2 & USGS-104 & 555 & Nitrogen, Nitrate/Nitrite & 0.905 & & & & MG/L & 06/15/2009 & & HCJ-154-09 \\
\hline GWA04702N2 & USGS-104 & 555 & Nitrogen, Nitrate/Nitrite & 0.905 & & & & MG/L & 06/15/2009 & & HCJ-154-09 \\
\hline GWA047012X & USGS-104 & 555 & Aluminum & 15 & & U & & UG/L & 06/15/2009 & & HCJ-160-09 \\
\hline GWA047012X & USGS-104 & 555 & Antimony & 1 & & $\mathrm{U}$ & & UG/L & 06/15/2009 & & HCJ-160-09 \\
\hline GWA047012X & USGS-104 & 555 & Arsenic & 3.85 & & B & $\mathrm{U}$ & UG/L & 06/15/2009 & & HCJ-160-09 \\
\hline GWA047012X & USGS-104 & 555 & Barium & 31.5 & & B & & UG/L & 06/15/2009 & & HCJ-160-09 \\
\hline GWA047012X & USGS-104 & 555 & Beryllium & 0.1 & & U & & UG/L & 06/15/2009 & & HCJ-160-09 \\
\hline GWA047012X & USGS-104 & 555 & Cadmium & 0.11 & & $\mathrm{U}$ & & UG/L & 06/15/2009 & & HCJ-160-09 \\
\hline GWA047012X & USGS-104 & 555 & Calcium & 35000 & & & & UG/L & 06/15/2009 & & HCJ-160-09 \\
\hline GWA047012X & USGS-104 & 555 & Chromium & 7.62 & & & & UG/L & 06/15/2009 & & HCJ-160-09 \\
\hline GWA047012X & USGS-104 & 555 & Cobalt & 0.101 & & B & & UG/L & 06/15/2009 & & HCJ-160-09 \\
\hline GWA047012X & USGS-104 & 555 & Copper & 0.461 & & B & $U$ & UG/L & 06/15/2009 & & HCJ-160-09 \\
\hline GWA047012X & USGS-104 & 555 & Iron & 94 & & B & & UG/L & 06/15/2009 & & HCJ-160-09 \\
\hline GWA047012X & USGS-104 & 555 & Lead & 0.5 & & $U$ & & UG/L & 06/15/2009 & & HCJ-160-09 \\
\hline GWA047012X & USGS-104 & 555 & Magnesium & 13300 & & & & UG/L & 06/15/2009 & & HCJ-160-09 \\
\hline GWA047012X & USGS-104 & 555 & Manganese & 1 & & $U$ & & UG/L & 06/15/2009 & & HCJ-160-09 \\
\hline GWA047012X & USGS-104 & 555 & Mercury & 0.066 & & U & & UG/L & 06/15/2009 & & HCJ-160-09 \\
\hline GWA047012X & USGS-104 & 555 & Nickel & 1.1 & & B & & UG/L & 06/15/2009 & & HCJ-160-09 \\
\hline GWA047012X & USGS-104 & 555 & Potassium & 2640 & & B & & UG/L & 06/15/2009 & & HCJ-160-09 \\
\hline GWA047012X & USGS-104 & 555 & Selenium & 1 & & $\mathrm{U}$ & & UG/L & 06/15/2009 & & HCJ-160-09 \\
\hline GWA047012X & USGS-104 & 555 & Silver & 0.2 & & U & & UG/L & 06/15/2009 & & HCJ-160-09 \\
\hline GWA047012X & USGS-104 & 555 & Sodium & 8960 & & & & UG/L & 06/15/2009 & & HCJ-160-09 \\
\hline GWA047012X & USGS-104 & 555 & Strontium & 209 & & & & UG/L & 06/15/2009 & & HCJ-160-09 \\
\hline GWA047012X & USGS-104 & 555 & Thallium & 0.315 & & B & $U$ & UG/L & 06/15/2009 & & HCJ-160-09 \\
\hline GWA047012X & USGS-104 & 555 & Uranium & 1.83 & & B & & UG/L & 06/15/2009 & & HCJ-160-09 \\
\hline GWA047012X & USGS-104 & 555 & Vanadium & 5.52 & & B & $U$ & UG/L & 06/15/2009 & & HCJ-160-09 \\
\hline GWA047012X & USGS-104 & 555 & Zinc & 3 & & $\mathrm{U}$ & & UG/L & 06/15/2009 & & HCJ-160-09 \\
\hline GWA047022X & USGS-104 & 555 & Aluminum & 15 & & U & & UG/L & 06/15/2009 & & HCJ-160-09 \\
\hline GWA047022X & USGS-104 & 555 & Antimony & 1 & & $U$ & & UG/L & 06/15/2009 & & HCJ-160-09 \\
\hline GWA047022X & USGS-104 & 555 & Arsenic & 2.57 & & B & $U$ & UG/L & 06/15/2009 & & HCJ-160-09 \\
\hline GWA047022X & USGS-104 & 555 & Barium & 31 & & B & & UG/L & 06/15/2009 & & HCJ-160-09 \\
\hline GWA047022X & USGS-104 & 555 & Beryllium & 0.1 & & U & & UG/L & 06/15/2009 & & HCJ-160-09 \\
\hline GWA047022X & USGS-104 & 555 & Cadmium & 0.11 & & U & & UG/L & 06/15/2009 & & HCJ-160-09 \\
\hline GWA047022X & USGS-104 & 555 & Calcium & 33400 & & & & UG/L & 06/15/2009 & & HCJ-160-09 \\
\hline GWA047022X & USGS-104 & 555 & Chromium & 9.91 & & & & UG/L & $06 / 15 / 2009$ & & HCJ-160-09 \\
\hline GWA047022X & USGS-104 & 555 & Cobalt & 0.1 & & $\mathrm{U}$ & & UG/L & 06/15/2009 & & HCJ-160-09 \\
\hline GWA047022X & USGS-104 & 555 & Copper & 0.349 & & B & $\mathrm{U}$ & UG/L & 06/15/2009 & & HCJ-160-09 \\
\hline GWA047022X & USGS-104 & 555 & Iron & 77.9 & & B & & UG/L & 06/15/2009 & & HCJ-160-09 \\
\hline GWA047022X & USGS-104 & 555 & Lead & 0.5 & & $\mathrm{U}$ & & UG/L & 06/15/2009 & & HCJ-160-09 \\
\hline GWA047022X & USGS-104 & 555 & Magnesium & 12500 & & & & UG/L & 06/15/2009 & & HCJ-160-09 \\
\hline
\end{tabular}


Table A-2. (continued).

\begin{tabular}{|c|c|c|c|c|c|c|c|c|c|c|c|}
\hline $\begin{array}{c}\text { Field Sample } \\
\text { Number }\end{array}$ & Location & Depth & Compound & $\begin{array}{c}\text { Sample } \\
\text { Result }\end{array}$ & $\begin{array}{c}\text { Sample } \\
\text { Error }\end{array}$ & $\begin{array}{c}\text { Result } \\
\text { Qualifier }\end{array}$ & $\begin{array}{c}\text { Validation } \\
\text { Flag }\end{array}$ & $\begin{array}{c}\text { Sample } \\
\text { Units }\end{array}$ & $\begin{array}{c}\text { Date Sample } \\
\text { Collected }\end{array}$ & MDA & L\&V Report Number \\
\hline GWA047022X & USGS-104 & 555 & Manganese & 1 & & $U$ & & UG/L & $06 / 15 / 2009$ & & HCJ-160-09 \\
\hline GWA047022X & USGS-104 & 555 & Mercury & 0.066 & & U & & UG/L & $06 / 15 / 2009$ & & HCJ-160-09 \\
\hline GWA047022X & USGS-104 & 555 & Nickel & 1.02 & & B & & UG/L & $06 / 15 / 2009$ & & HCJ-160-09 \\
\hline GWA047022X & USGS-104 & 555 & Potassium & 2500 & & B & & UG/L & $06 / 15 / 2009$ & & HCJ-160-09 \\
\hline GWA047022X & USGS-104 & 555 & Selenium & 1.23 & & B & & UG/L & $06 / 15 / 2009$ & & HCJ-160-09 \\
\hline GWA047022X & USGS-104 & 555 & Silver & 0.2 & & $\mathrm{U}$ & & UG/L & $06 / 15 / 2009$ & & HCJ-160-09 \\
\hline GWA047022X & USGS-104 & 555 & Sodium & 8160 & & & & UG/L & $06 / 15 / 2009$ & & HCJ-160-09 \\
\hline GWA047022X & USGS-104 & 555 & Strontium & 207 & & & & UG/L & $06 / 15 / 2009$ & & HCJ-160-09 \\
\hline GWA047022X & USGS-104 & 555 & Thallium & 0.3 & & U & & UG/L & $06 / 15 / 2009$ & & HCJ-160-09 \\
\hline GWA047022X & USGS-104 & 555 & Uranium & 1.83 & & B & & UG/L & $06 / 15 / 2009$ & & HCJ-160-09 \\
\hline GWA047022X & USGS-104 & 555 & Vanadium & 6.68 & & B & U & UG/L & $06 / 15 / 2009$ & & HCJ-160-09 \\
\hline GWA047022X & USGS-104 & 555 & Zinc & 3 & & $U$ & & UG/L & $06 / 15 / 2009$ & & HCJ-160-09 \\
\hline GWA04701VL & USGS-104 & 555 & Dichlorodifluoromethane & 1 & & $\mathrm{U}$ & & UG/L & $06 / 15 / 2009$ & & HCJ-153-09 \\
\hline GWA04701VL & USGS-104 & 555 & Chloromethane & 1 & & $\mathrm{U}$ & & UG/L & $06 / 15 / 2009$ & & HCJ-153-09 \\
\hline GWA04701VL & USGS-104 & 555 & Vinyl Chloride & 1 & & U & & UG/L & $06 / 15 / 2009$ & & HCJ-153-09 \\
\hline GWA04701VL & USGS-104 & 555 & Bromomethane & 1 & & U & & UG/L & $06 / 15 / 2009$ & & HCJ-153-09 \\
\hline GWA04701VL & USGS-104 & 555 & Chloroethane & 1 & & $\mathrm{U}$ & & UG/L & $06 / 15 / 2009$ & & HCJ-153-09 \\
\hline GWA04701VL & USGS-104 & 555 & Trichlorofluoromethane & 1 & & $\mathrm{U}$ & & UG/L & $06 / 15 / 2009$ & & HCJ-153-09 \\
\hline GWA04701VL & USGS-104 & 555 & Acetone & 5 & & U & & UG/L & $06 / 15 / 2009$ & & HCJ-153-09 \\
\hline GWA04701VL & USGS-104 & 555 & 1,1-Dichloroethene & 1 & & $u$ & & UG/L & $06 / 15 / 2009$ & & HCJ-153-09 \\
\hline GWA04701VL & USGS-104 & 555 & Methyl acetate & 5 & & $\mathrm{U}$ & & UG/L & $06 / 15 / 2009$ & & HCJ-153-09 \\
\hline GWA04701VL & USGS-104 & 555 & Methylene Chloride & 5 & & U & & UG/L & $06 / 15 / 2009$ & & HCJ-153-09 \\
\hline GWA04701VL & USGS-104 & 555 & Carbon disulfide & 5 & & U & & UG/L & $06 / 15 / 2009$ & & HCJ-153-09 \\
\hline GWA04701VL & USGS-104 & 555 & Tert-butyl methyl ether & 1 & & U & & UG/L & $06 / 15 / 2009$ & & HCJ-153-09 \\
\hline GWA04701VL & USGS-104 & 555 & trans-1,2-Dichloroethylene & 1 & & $\mathrm{U}$ & & UG/L & $06 / 15 / 2009$ & & HCJ-153-09 \\
\hline GWA04701VL & USGS-104 & 555 & 1,1-Dichloroethane & 1 & & U & & UG/L & $06 / 15 / 2009$ & & HCJ-153-09 \\
\hline GWA04701VL & USGS-104 & 555 & 2-Butanone & 5 & & $\mathrm{U}$ & & UG/L & $06 / 15 / 2009$ & & HCJ-153-09 \\
\hline GWA04701VL & USGS-104 & 555 & cis-1,2-Dichloroethylene & 1 & & $\mathrm{U}$ & & UG/L & $06 / 15 / 2009$ & & HCJ-153-09 \\
\hline GWA04701VL & USGS-104 & 555 & Chloroform & 1 & & U & & UG/L & $06 / 15 / 2009$ & & HCJ-153-09 \\
\hline GWA04701VL & USGS-104 & 555 & 1,1,1-Trichloroethane & 1 & & U & & UG/L & $06 / 15 / 2009$ & & HCJ-153-09 \\
\hline GWA04701VL & USGS-104 & 555 & Cyclohexane & 1 & & $u$ & & UG/L & $06 / 15 / 2009$ & & HCJ-153-09 \\
\hline GWA04701VL & USGS-104 & 555 & Carbon tetrachloride & 1 & & $\mathrm{U}$ & & UG/L & $06 / 15 / 2009$ & & HCJ-153-09 \\
\hline GWA04701VL & USGS-104 & 555 & 1,2-Dichloroethane & 1 & & U & & UG/L & $06 / 15 / 2009$ & & HCJ-153-09 \\
\hline GWA04701VL & USGS-104 & 555 & Benzene & 1 & & $u$ & & UG/L & $06 / 15 / 2009$ & & HCJ-153-09 \\
\hline GWA04701VL & USGS-104 & 555 & Trichloroethylene & 1 & & $\mathrm{u}$ & & UG/L & $06 / 15 / 2009$ & & HCJ-153-09 \\
\hline GWA04701VL & USGS-104 & 555 & 1,2-Dichloropropane & 1 & & U & & UG/L & $06 / 15 / 2009$ & & HCJ-153-09 \\
\hline GWA04701VL & USGS-104 & 555 & Methyl cyclohexane & 1 & & U & & UG/L & $06 / 15 / 2009$ & & HCJ-153-09 \\
\hline GWA04701VL & USGS-104 & 555 & Bromodichloromethane & 1 & & $u$ & & UG/L & $06 / 15 / 2009$ & & HCJ-153-09 \\
\hline GWA04701VL & USGS-104 & 555 & 4-Methyl-2-pentanone & 5 & & $\mathrm{u}$ & & UG/L & $06 / 15 / 2009$ & & HCJ-153-09 \\
\hline GWA04701VL & USGS-104 & 555 & cis-1,3-Dichloropropylene & 1 & & $\mathrm{U}$ & & UG/L & $06 / 15 / 2009$ & & HCJ-153-09 \\
\hline GWA04701VL & USGS-104 & 555 & Toluene & 1 & & $\mathrm{U}$ & & UG/L & $06 / 15 / 2009$ & & HCJ-153-09 \\
\hline GWA04701VL & USGS-104 & 555 & trans-1,3-Dichloropropylene & 1 & & $\mathrm{U}$ & & UG/L & $06 / 15 / 2009$ & & HCJ-153-09 \\
\hline GWA04701VL & USGS-104 & 555 & 1,1,2-Trichloroethane & 1 & & $u$ & & UG/L & $06 / 15 / 2009$ & & HCJ-153-09 \\
\hline GWA04701VL & USGS-104 & 555 & 2-Hexanone & 5 & & $\mathrm{U}$ & & UG/L & $06 / 15 / 2009$ & & HCJ-153-09 \\
\hline
\end{tabular}


Table A-2. (continued).

\begin{tabular}{|c|c|c|c|c|c|c|c|c|c|c|c|}
\hline $\begin{array}{c}\text { Field Sample } \\
\text { Number }\end{array}$ & Location & Depth & Compound & $\begin{array}{c}\text { Sample } \\
\text { Result }\end{array}$ & $\begin{array}{c}\text { Sample } \\
\text { Error }\end{array}$ & $\begin{array}{c}\text { Result } \\
\text { Qualifier }\end{array}$ & $\begin{array}{c}\text { Validation } \\
\text { Flag }\end{array}$ & $\begin{array}{c}\text { Sample } \\
\text { Units }\end{array}$ & $\begin{array}{c}\text { Date Sample } \\
\text { Collected }\end{array}$ & MDA & L\&V Report Number \\
\hline GWA04701VL & USGS-104 & 555 & Tetrachloroethylene & 1 & & $U$ & & UG/L & $06 / 15 / 2009$ & & HCJ-153-09 \\
\hline GWA04701VL & USGS-104 & 555 & Dibromochloromethane & 1 & & U & & UG/L & $06 / 15 / 2009$ & & HCJ-153-09 \\
\hline GWA04701VL & USGS-104 & 555 & 1,2-Dibromoethane & 1 & & $\mathrm{u}$ & & UG/L & $06 / 15 / 2009$ & & HCJ-153-09 \\
\hline GWA04701VL & USGS-104 & 555 & Chlorobenzene & 1 & & $\mathrm{U}$ & & UG/L & $06 / 15 / 2009$ & & HCJ-153-09 \\
\hline GWA04701VL & USGS-104 & 555 & Ethylbenzene & 1 & & U & & UG/L & $06 / 15 / 2009$ & & HCJ-153-09 \\
\hline GWA04701VL & USGS-104 & 555 & Styrene & 1 & & $\mathrm{u}$ & & UG/L & $06 / 15 / 2009$ & & HCJ-153-09 \\
\hline GWA04701VL & USGS-104 & 555 & Bromoform & 1 & & $\mathrm{U}$ & & UG/L & $06 / 15 / 2009$ & & HCJ-153-09 \\
\hline GWA04701VL & USGS-104 & 555 & 1,1,2,2-Tetrachloroethane & 1 & & U & & UG/L & $06 / 15 / 2009$ & & HCJ-153-09 \\
\hline GWA04701VL & USGS-104 & 555 & Isopropylbenzene & 1 & & U & & UG/L & $06 / 15 / 2009$ & & HCJ-153-09 \\
\hline GWA04701VL & USGS-104 & 555 & 1,3-Dichlorobenzene & 1 & & $\mathrm{U}$ & & UG/L & $06 / 15 / 2009$ & & HCJ-153-09 \\
\hline GWA04701VL & USGS-104 & 555 & 1,4-Dichlorobenzene & 1 & & $\mathrm{U}$ & & UG/L & $06 / 15 / 2009$ & & HCJ-153-09 \\
\hline GWA04701VL & USGS-104 & 555 & 1,2-Dibromo-3-chloropropane & 1 & & U & & UG/L & $06 / 15 / 2009$ & & HCJ-153-09 \\
\hline GWA04701VL & USGS-104 & 555 & Trichlorotrifluoroethane & 5 & & $u$ & & UG/L & $06 / 15 / 2009$ & & HCJ-153-09 \\
\hline GWA04701VL & USGS-104 & 555 & Xylene (Total) & 1 & & $\mathrm{u}$ & & UG/L & $06 / 15 / 2009$ & & HCJ-153-09 \\
\hline GWA04701VL & USGS-104 & 555 & 1,2,4-Trichlorobenzene & 1 & & U & & UG/L & $06 / 15 / 2009$ & & HCJ-153-09 \\
\hline GWA04701VL & USGS-104 & 555 & 1,2-Dichlorobenzene & 1 & & U & & UG/L & $06 / 15 / 2009$ & & HCJ-153-09 \\
\hline GWA04702VL & USGS-104 & 555 & Dichlorodifluoromethane & 1 & & $\mathrm{u}$ & & UG/L & $06 / 15 / 2009$ & & HCJ-153-09 \\
\hline GWA04702VL & USGS-104 & 555 & Chloromethane & 1 & & $\mathrm{U}$ & & UG/L & $06 / 15 / 2009$ & & HCJ-153-09 \\
\hline GWA04702VL & USGS-104 & 555 & Vinyl Chloride & 1 & & U & & UG/L & $06 / 15 / 2009$ & & HCJ-153-09 \\
\hline GWA04702VL & USGS-104 & 555 & Bromomethane & 1 & & $U$ & & UG/L & $06 / 15 / 2009$ & & HCJ-153-09 \\
\hline GWA04702VL & USGS-104 & 555 & Chloroethane & 1 & & $\mathrm{u}$ & & UG/L & $06 / 15 / 2009$ & & HCJ-153-09 \\
\hline GWA04702VL & USGS-104 & 555 & Trichlorofluoromethane & 1 & & U & & UG/L & $06 / 15 / 2009$ & & HCJ-153-09 \\
\hline GWA04702VL & USGS-104 & 555 & Acetone & 5 & & U & & UG/L & $06 / 15 / 2009$ & & HCJ-153-09 \\
\hline GWA04702VL & USGS-104 & 555 & 1,1-Dichloroethene & 1 & & U & & UG/L & $06 / 15 / 2009$ & & HCJ-153-09 \\
\hline GWA04702VL & USGS-104 & 555 & Methyl acetate & 5 & & $\mathrm{U}$ & & UG/L & $06 / 15 / 2009$ & & HCJ-153-09 \\
\hline GWA04702VL & USGS-104 & 555 & Methylene Chloride & 5 & & U & & UG/L & $06 / 15 / 2009$ & & HCJ-153-09 \\
\hline GWA04702VL & USGS-104 & 555 & Carbon disulfide & 5 & & $\mathrm{U}$ & & UG/L & $06 / 15 / 2009$ & & HCJ-153-09 \\
\hline GWA04702VL & USGS-104 & 555 & Tert-butyl methyl ether & 1 & & $\mathrm{U}$ & & UG/L & $06 / 15 / 2009$ & & HCJ-153-09 \\
\hline GWA04702VL & USGS-104 & 555 & trans-1,2-Dichloroethylene & 1 & & U & & UG/L & $06 / 15 / 2009$ & & HCJ-153-09 \\
\hline GWA04702VL & USGS-104 & 555 & 1,1-Dichloroethane & 1 & & U & & UG/L & $06 / 15 / 2009$ & & HCJ-153-09 \\
\hline GWA04702VL & USGS-104 & 555 & 2-Butanone & 5 & & 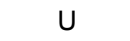 & & UG/L & $06 / 15 / 2009$ & & HCJ-153-09 \\
\hline GWA04702VL & USGS-104 & 555 & cis-1,2-Dichloroethylene & 1 & & $\mathrm{U}$ & & UG/L & $06 / 15 / 2009$ & & HCJ-153-09 \\
\hline GWA04702VL & USGS-104 & 555 & Chloroform & 1 & & $\mathrm{U}$ & & UG/L & $06 / 15 / 2009$ & & HCJ-153-09 \\
\hline GWA04702VL & USGS-104 & 555 & 1,1,1-Trichloroethane & 1 & & $U$ & & UG/L & $06 / 15 / 2009$ & & HCJ-153-09 \\
\hline GWA04702VL & USGS-104 & 555 & Cyclohexane & 1 & & $\mathrm{u}$ & & UG/L & $06 / 15 / 2009$ & & HCJ-153-09 \\
\hline GWA04702VL & USGS-104 & 555 & Carbon tetrachloride & 1 & & $\mathrm{U}$ & & UG/L & $06 / 15 / 2009$ & & HCJ-153-09 \\
\hline GWA04702VL & USGS-104 & 555 & 1,2-Dichloroethane & 1 & & U & & UG/L & $06 / 15 / 2009$ & & HCJ-153-09 \\
\hline GWA04702VL & USGS-104 & 555 & Benzene & 1 & & $U$ & & UG/L & $06 / 15 / 2009$ & & HCJ-153-09 \\
\hline GWA04702VL & USGS-104 & 555 & Trichloroethylene & 1 & & $\mathrm{U}$ & & UG/L & $06 / 15 / 2009$ & & HCJ-153-09 \\
\hline GWA04702VL & USGS-104 & 555 & 1,2-Dichloropropane & 1 & & $\mathrm{U}$ & & UG/L & $06 / 15 / 2009$ & & HCJ-153-09 \\
\hline GWA04702VL & USGS-104 & 555 & Methyl cyclohexane & 1 & & $\mathrm{U}$ & & UG/L & $06 / 15 / 2009$ & & HCJ-153-09 \\
\hline GWA04702VL & USGS-104 & 555 & Bromodichloromethane & 1 & & $\mathrm{U}$ & & UG/L & $06 / 15 / 2009$ & & HCJ-153-09 \\
\hline GWA04702VL & USGS-104 & 555 & 4-Methyl-2-pentanone & 5 & & 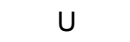 & & UG/L & $06 / 15 / 2009$ & & HCJ-153-09 \\
\hline GWA04702VL & USGS-104 & 555 & cis-1,3-Dichloropropylene & 1 & & U & & UG/L & $06 / 15 / 2009$ & & HCJ-153-09 \\
\hline
\end{tabular}


Table A-2. (continued).

\begin{tabular}{|c|c|c|c|c|c|c|c|c|c|c|c|}
\hline $\begin{array}{c}\text { Field Sample } \\
\text { Number }\end{array}$ & Location & Depth & Compound & $\begin{array}{c}\text { Sample } \\
\text { Result }\end{array}$ & $\begin{array}{l}\text { Sample } \\
\text { Error }\end{array}$ & $\begin{array}{c}\text { Result } \\
\text { Qualifier }\end{array}$ & $\begin{array}{c}\text { Validation } \\
\text { Flag }\end{array}$ & $\begin{array}{c}\text { Sample } \\
\text { Units }\end{array}$ & $\begin{array}{c}\text { Date Sample } \\
\text { Collected }\end{array}$ & MDA & L\&V Report Number \\
\hline GWA04702VL & USGS-104 & 555 & Toluene & 1 & & $U$ & & UG/L & $06 / 15 / 2009$ & & HCJ-153-09 \\
\hline GWA04702VL & USGS-104 & 555 & trans-1,3-Dichloropropylene & 1 & & U & & UG/L & $06 / 15 / 2009$ & & HCJ-153-09 \\
\hline GWA04702VL & USGS-104 & 555 & 1,1,2-Trichloroethane & 1 & & $\mathrm{u}$ & & UG/L & $06 / 15 / 2009$ & & HCJ-153-09 \\
\hline GWA04702VL & USGS-104 & 555 & 2-Hexanone & 5 & & $\mathrm{U}$ & & UG/L & $06 / 15 / 2009$ & & HCJ-153-09 \\
\hline GWA04702VL & USGS-104 & 555 & Tetrachloroethylene & 1 & & U & & UG/L & $06 / 15 / 2009$ & & HCJ-153-09 \\
\hline GWA04702VL & USGS-104 & 555 & Dibromochloromethane & 1 & & $\mathrm{u}$ & & UG/L & $06 / 15 / 2009$ & & HCJ-153-09 \\
\hline GWA04702VL & USGS-104 & 555 & 1,2-Dibromoethane & 1 & & $\mathrm{U}$ & & UG/L & $06 / 15 / 2009$ & & HCJ-153-09 \\
\hline GWA04702VL & USGS-104 & 555 & Chlorobenzene & 1 & & U & & UG/L & $06 / 15 / 2009$ & & HCJ-153-09 \\
\hline GWA04702VL & USGS-104 & 555 & Ethylbenzene & 1 & & U & & UG/L & $06 / 15 / 2009$ & & HCJ-153-09 \\
\hline GWA04702VL & USGS-104 & 555 & Styrene & 1 & & $\mathrm{u}$ & & UG/L & $06 / 15 / 2009$ & & HCJ-153-09 \\
\hline GWA04702VL & USGS-104 & 555 & Bromoform & 1 & & $\mathrm{U}$ & & UG/L & $06 / 15 / 2009$ & & HCJ-153-09 \\
\hline GWA04702VL & USGS-104 & 555 & 1,1,2,2-Tetrachloroethane & 1 & & U & & UG/L & $06 / 15 / 2009$ & & HCJ-153-09 \\
\hline GWA04702VL & USGS-104 & 555 & Isopropylbenzene & 1 & & U & & UG/L & $06 / 15 / 2009$ & & HCJ-153-09 \\
\hline GWA04702VL & USGS-104 & 555 & 1,3-Dichlorobenzene & 1 & & $\mathrm{u}$ & & UG/L & $06 / 15 / 2009$ & & HCJ-153-09 \\
\hline GWA04702VL & USGS-104 & 555 & 1,4-Dichlorobenzene & 1 & & U & & UG/L & $06 / 15 / 2009$ & & HCJ-153-09 \\
\hline GWA04702VL & USGS-104 & 555 & 1,2-Dibromo-3-chloropropane & 1 & & U & & UG/L & $06 / 15 / 2009$ & & HCJ-153-09 \\
\hline GWA04702VL & USGS-104 & 555 & Trichlorotrifluoroethane & 5 & & $\mathrm{u}$ & & UG/L & $06 / 15 / 2009$ & & HCJ-153-09 \\
\hline GWA04702VL & USGS-104 & 555 & Xylene (Total) & 1 & & $\mathrm{U}$ & & UG/L & $06 / 15 / 2009$ & & HCJ-153-09 \\
\hline GWA04702VL & USGS-104 & 555 & 1,2,4-Trichlorobenzene & 1 & & U & & UG/L & $06 / 15 / 2009$ & & HCJ-153-09 \\
\hline GWA04702VL & USGS-104 & 555 & 1,2-Dichlorobenzene & 1 & & U & & UG/L & $06 / 15 / 2009$ & & HCJ-153-09 \\
\hline GWA04701R8 & USGS-104 & 555 & Tritium & $7.53 \mathrm{E}+02$ & $1.32 \mathrm{E}+02$ & & & $\mathrm{PCl} / \mathrm{L}$ & $06 / 15 / 2009$ & $3.06 \mathrm{E}+02$ & BAM-119-09 \\
\hline GWA04702R8 & USGS-104 & 555 & Tritium & $5.67 \mathrm{E}+02$ & $1.18 E+02$ & & & $\mathrm{PCl} / \mathrm{L}$ & $06 / 15 / 2009$ & $3.05 E+02$ & BAM-119-09 \\
\hline GWA04701RH & USGS-104 & 555 & Gross Alpha & $-1.59 \mathrm{E}-01$ & 3.33E-01 & & $U$ & $\mathrm{PCl} / \mathrm{L}$ & $06 / 15 / 2009$ & $1.71 \mathrm{E}+00$ & BAM-120-09 \\
\hline GWA04701RH & USGS-104 & 555 & Gross Beta & $1.62 \mathrm{E}+00$ & $5.30 \mathrm{E}-01$ & & & $\mathrm{PCl} / \mathrm{L}$ & $06 / 15 / 2009$ & $1.43 \mathrm{E}+00$ & BAM-120-09 \\
\hline GWA04702RH & USGS-104 & 555 & Gross Alpha & $4.62 \mathrm{E}+00$ & $1.23 E+00$ & & & $\mathrm{PCl} / \mathrm{L}$ & $06 / 15 / 2009$ & $2.38 \mathrm{E}+00$ & BAM-120-09 \\
\hline GWA04702RH & USGS-104 & 555 & Gross Beta & $3.35 \mathrm{E}+00$ & $9.95 \mathrm{E}-01$ & & & $\mathrm{PCl} / \mathrm{L}$ & $06 / 15 / 2009$ & $2.81 \mathrm{E}+00$ & BAM-120-09 \\
\hline GWA04701RH & USGS-104 & 555 & Strontium-90 & 1.31E-02 & 1.35E-01 & & $u$ & $\mathrm{PCl} / \mathrm{L}$ & $06 / 15 / 2009$ & 4.70E-01 & BAM-120-09 \\
\hline GWA04702RH & USGS-104 & 555 & Strontium-90 & 3.01E-01 & $1.08 \mathrm{E}-01$ & & UJ & $\mathrm{PCl} / \mathrm{L}$ & $06 / 15 / 2009$ & 3.21E-01 & BAM-120-09 \\
\hline GWA04701RH & USGS-104 & 555 & Technetium-99 & $-3.09 \mathrm{E}+00$ & $1.60 \mathrm{E}+00$ & & $U$ & $\mathrm{PCl} / \mathrm{L}$ & $06 / 15 / 2009$ & $5.89 \mathrm{E}+00$ & BAM-120-09 \\
\hline GWA04702RH & USGS-104 & 555 & Technetium-99 & $-1.88 \mathrm{E}+00$ & $1.61 \mathrm{E}+00$ & & $\mathrm{U}$ & $\mathrm{PCl} / \mathrm{L}$ & $06 / 15 / 2009$ & $5.82 \mathrm{E}+00$ & BAM-120-09 \\
\hline GWA04702UX & USGS-104 & 555 & lodine-129 & $-5.34 \mathrm{E}-04$ & 2.69E-02 & & $\mathrm{U}$ & $\mathrm{PCl} / \mathrm{L}$ & $06 / 15 / 2009$ & $9.00 \mathrm{E}-02$ & BAM-118-09 \\
\hline GWA04701UX & USGS-104 & 555 & lodine-129 & 1.98E-02 & $3.53 \mathrm{E}-02$ & & $\mathrm{U}$ & $\mathrm{PCl} / \mathrm{L}$ & $06 / 15 / 2009$ & 1.19E-01 & BAM-118-09 \\
\hline GWA04801VL & USGS-106 & 584 & 1,1,1-Trichloroethane & 1 & & $U$ & & UG/L & $06 / 10 / 2009$ & & HCJ-148-09 \\
\hline GWA04802VL & USGS-106 & 584 & 1,1,1-Trichloroethane & 1 & & U & & UG/L & 06/10/2009 & & HCJ-148-09 \\
\hline GWA04801VL & USGS-106 & 584 & 1,1,2,2-Tetrachloroethane & 1 & & $\mathrm{u}$ & & UG/L & $06 / 10 / 2009$ & & HCJ-148-09 \\
\hline GWA04802VL & USGS-106 & 584 & 1,1,2,2-Tetrachloroethane & 1 & & U & & UG/L & $06 / 10 / 2009$ & & HCJ-148-09 \\
\hline GWA04801VL & USGS-106 & 584 & 1,1,2-Trichloroethane & 1 & & U & & UG/L & $06 / 10 / 2009$ & & HCJ-148-09 \\
\hline GWA04802VL & USGS-106 & 584 & 1,1,2-Trichloroethane & 1 & & 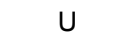 & & UG/L & $06 / 10 / 2009$ & & HCJ-148-09 \\
\hline GWA04801VL & USGS-106 & 584 & 1,1-Dichloroethane & 1 & & $\mathrm{u}$ & & UG/L & $06 / 10 / 2009$ & & HCJ-148-09 \\
\hline GWA04802VL & USGS-106 & 584 & 1,1-Dichloroethane & 1 & & $U$ & & UG/L & $06 / 10 / 2009$ & & HCJ-148-09 \\
\hline GWA04801VL & USGS-106 & 584 & 1,1-Dichloroethene & 1 & & $U$ & & UG/L & 06/10/2009 & & HCJ-148-09 \\
\hline GWA04802VL & USGS-106 & 584 & 1,1-Dichloroethene & 1 & & $\mathrm{U}$ & & UG/L & $06 / 10 / 2009$ & & HCJ-148-09 \\
\hline GWA04801VL & USGS-106 & 584 & 1,2,4-Trichlorobenzene & 1 & & U & & UG/L & $06 / 10 / 2009$ & & HCJ-148-09 \\
\hline GWA04802VL & USGS-106 & 584 & 1,2,4-Trichlorobenzene & 1 & & U & & UG/L & $06 / 10 / 2009$ & & HCJ-148-09 \\
\hline
\end{tabular}


Table A-2. (continued).

\begin{tabular}{|c|c|c|c|c|c|c|c|c|c|c|c|}
\hline $\begin{array}{c}\text { Field Sample } \\
\text { Number }\end{array}$ & Location & Depth & Compound & $\begin{array}{c}\text { Sample } \\
\text { Result }\end{array}$ & $\begin{array}{c}\text { Sample } \\
\text { Error }\end{array}$ & $\begin{array}{c}\text { Result } \\
\text { Qualifier }\end{array}$ & $\begin{array}{c}\text { Validation } \\
\text { Flag }\end{array}$ & $\begin{array}{c}\text { Sample } \\
\text { Units }\end{array}$ & $\begin{array}{c}\text { Date Sample } \\
\text { Collected }\end{array}$ & MDA & L\&V Report Number \\
\hline GWA04801VL & USGS-106 & 584 & 1,2-Dibromo-3-chloropropane & 1 & & $U$ & & UG/L & $06 / 10 / 2009$ & & HCJ-148-09 \\
\hline GWA04802VL & USGS-106 & 584 & 1,2-Dibromo-3-chloropropane & 1 & & U & & UG/L & 06/10/2009 & & HCJ-148-09 \\
\hline GWA04801VL & USGS-106 & 584 & 1,2-Dibromoethane & 1 & & $\mathrm{u}$ & & UG/L & $06 / 10 / 2009$ & & HCJ-148-09 \\
\hline GWA04802VL & USGS-106 & 584 & 1,2-Dibromoethane & 1 & & $\mathrm{U}$ & & UG/L & $06 / 10 / 2009$ & & HCJ-148-09 \\
\hline GWA04801VL & USGS-106 & 584 & 1,2-Dichlorobenzene & 1 & & U & & UG/L & $06 / 10 / 2009$ & & HCJ-148-09 \\
\hline GWA04802VL & USGS-106 & 584 & 1,2-Dichlorobenzene & 1 & & $\mathrm{u}$ & & UG/L & $06 / 10 / 2009$ & & HCJ-148-09 \\
\hline GWA04801VL & USGS-106 & 584 & 1,2-Dichloroethane & 1 & & $\mathrm{U}$ & & UG/L & $06 / 10 / 2009$ & & HCJ-148-09 \\
\hline GWA04802VL & USGS-106 & 584 & 1,2-Dichloroethane & 1 & & U & & UG/L & $06 / 10 / 2009$ & & HCJ-148-09 \\
\hline GWA04801VL & USGS-106 & 584 & 1,2-Dichloropropane & 1 & & U & & UG/L & $06 / 10 / 2009$ & & HCJ-148-09 \\
\hline GWA04802VL & USGS-106 & 584 & 1,2-Dichloropropane & 1 & & $\mathrm{u}$ & & UG/L & $06 / 10 / 2009$ & & HCJ-148-09 \\
\hline GWA04801VL & USGS-106 & 584 & 1,3-Dichlorobenzene & 1 & & $\mathrm{U}$ & & UG/L & $06 / 10 / 2009$ & & HCJ-148-09 \\
\hline GWA04802VL & USGS-106 & 584 & 1,3-Dichlorobenzene & 1 & & $U$ & & UG/L & $06 / 10 / 2009$ & & HCJ-148-09 \\
\hline GWA04801VL & USGS-106 & 584 & 1,4-Dichlorobenzene & 1 & & U & & UG/L & 06/10/2009 & & HCJ-148-09 \\
\hline GWA04802VL & USGS-106 & 584 & 1,4-Dichlorobenzene & 1 & & $\mathrm{u}$ & & UG/L & $06 / 10 / 2009$ & & HCJ-148-09 \\
\hline GWA04801VL & USGS-106 & 584 & 2-Butanone & 5 & & U & & UG/L & $06 / 10 / 2009$ & & HCJ-148-09 \\
\hline GWA04802VL & USGS-106 & 584 & 2-Butanone & 5 & & U & & UG/L & $06 / 10 / 2009$ & & HCJ-148-09 \\
\hline GWA04801VL & USGS-106 & 584 & 2-Hexanone & 5 & & $\mathrm{U}$ & & UG/L & $06 / 10 / 2009$ & & HCJ-148-09 \\
\hline GWA04802VL & USGS-106 & 584 & 2-Hexanone & 5 & & $\mathrm{U}$ & & UG/L & $06 / 10 / 2009$ & & HCJ-148-09 \\
\hline GWA04801VL & USGS-106 & 584 & 4-Methyl-2-pentanone & 5 & & U & & UG/L & $06 / 10 / 2009$ & & HCJ-148-09 \\
\hline GWA04802VL & USGS-106 & 584 & 4-Methyl-2-pentanone & 5 & & $\mathrm{U}$ & & UG/L & $06 / 10 / 2009$ & & HCJ-148-09 \\
\hline GWA04801VL & USGS-106 & 584 & Acetone & 5 & & $\mathrm{U}$ & & UG/L & $06 / 10 / 2009$ & & HCJ-148-09 \\
\hline GWA04802VL & USGS-106 & 584 & Acetone & 5 & & U & & UG/L & $06 / 10 / 2009$ & & HCJ-148-09 \\
\hline GWA04802A1 & USGS-106 & 584 & Alkalinity, Total as $\mathrm{CaCO} 3$ & 155 & & & & $M G / L$ & $06 / 10 / 2009$ & & HCJ-157-09 \\
\hline GWA04801A1 & USGS-106 & 584 & Alkalinity, Total as $\mathrm{CaCO} 3$ & 155 & & & & $M G / L$ & $06 / 10 / 2009$ & & HCJ-157-09 \\
\hline GWA048012X & USGS-106 & 584 & Aluminum & 15 & & $\mathrm{U}$ & & UG/L & $06 / 10 / 2009$ & & HCJ-161-09 \\
\hline GWA048022X & USGS-106 & 584 & Aluminum & 15 & & U & & UG/L & $06 / 10 / 2009$ & & HCJ-161-09 \\
\hline GWA048012X & USGS-106 & 584 & Antimony & 1 & & $\mathrm{U}$ & & UG/L & 06/10/2009 & & HCJ-161-09 \\
\hline GWA048022X & USGS-106 & 584 & Antimony & 1 & & $\mathrm{U}$ & & UG/L & $06 / 10 / 2009$ & & HCJ-161-09 \\
\hline GWA048012X & USGS-106 & 584 & Arsenic & 1.6 & & U & & UG/L & $06 / 10 / 2009$ & & HCJ-161-09 \\
\hline GWA048022X & USGS-106 & 584 & Arsenic & 1.6 & & U & & UG/L & $06 / 10 / 2009$ & & HCJ-161-09 \\
\hline GWA048012X & USGS-106 & 584 & Barium & 46.8 & & $\mathrm{~B}$ & & UG/L & $06 / 10 / 2009$ & & HCJ-161-09 \\
\hline GWA048022X & USGS-106 & 584 & Barium & 47 & & B & & UG/L & $06 / 10 / 2009$ & & HCJ-161-09 \\
\hline GWA04801VL & USGS-106 & 584 & Benzene & 1 & & U & & UG/L & $06 / 10 / 2009$ & & HCJ-148-09 \\
\hline GWA04802VL & USGS-106 & 584 & Benzene & 1 & & $U$ & & UG/L & $06 / 10 / 2009$ & & HCJ-148-09 \\
\hline GWA048012X & USGS-106 & 584 & Beryllium & 0.1 & & $\mathrm{u}$ & & UG/L & $06 / 10 / 2009$ & & HCJ-161-09 \\
\hline GWA048022X & USGS-106 & 584 & Beryllium & 0.1 & & $\mathrm{U}$ & & UG/L & $06 / 10 / 2009$ & & HCJ-161-09 \\
\hline GWA04801AN & USGS-106 & 584 & Bromide & 0 & & U & & $M G / L$ & $06 / 10 / 2009$ & & HCJ-159-09 \\
\hline GWA04802AN & USGS-106 & 584 & Bromide & 0 & & $u$ & & $M G / L$ & $06 / 10 / 2009$ & & HCJ-159-09 \\
\hline GWA04801VL & USGS-106 & 584 & Bromodichloromethane & 1 & & $\mathrm{U}$ & & UG/L & $06 / 10 / 2009$ & & HCJ-148-09 \\
\hline GWA04802VL & USGS-106 & 584 & Bromodichloromethane & 1 & & $\mathrm{U}$ & & UG/L & 06/10/2009 & & HCJ-148-09 \\
\hline GWA04801VL & USGS-106 & 584 & Bromoform & 1 & & $\mathrm{U}$ & & UG/L & 06/10/2009 & & HCJ-148-09 \\
\hline GWA04802VL & USGS-106 & 584 & Bromoform & 1 & & $\mathrm{U}$ & & UG/L & 06/10/2009 & & HCJ-148-09 \\
\hline GWA04801VL & USGS-106 & 584 & Bromomethane & 1 & & $u$ & & UG/L & $06 / 10 / 2009$ & & HCJ-148-09 \\
\hline GWA04802VL & USGS-106 & 584 & Bromomethane & 1 & & U & & UG/L & 06/10/2009 & & HCJ-148-09 \\
\hline
\end{tabular}


Table A-2. (continued).

\begin{tabular}{|c|c|c|c|c|c|c|c|c|c|c|c|}
\hline $\begin{array}{c}\text { Field Sample } \\
\text { Number }\end{array}$ & Location & Depth & Compound & $\begin{array}{l}\text { Sample } \\
\text { Result }\end{array}$ & $\begin{array}{c}\text { Sample } \\
\text { Error }\end{array}$ & $\begin{array}{c}\text { Result } \\
\text { Qualifier }\end{array}$ & $\begin{array}{c}\text { Validation } \\
\text { Flag }\end{array}$ & $\begin{array}{c}\text { Sample } \\
\text { Units }\end{array}$ & $\begin{array}{c}\text { Date Sample } \\
\text { Collected }\end{array}$ & MDA & L\&V Report Number \\
\hline GWA048012X & USGS-106 & 584 & Cadmium & 0.288 & & $B$ & & UG/L & $06 / 10 / 2009$ & & HCJ-161-09 \\
\hline GWA048022X & USGS-106 & 584 & Cadmium & 0.24 & & B & & UG/L & 06/10/2009 & & HCJ-161-09 \\
\hline GWA048012X & USGS-106 & 584 & Calcium & 43300 & & & & UG/L & 06/10/2009 & & HCJ-161-09 \\
\hline GWA048022X & USGS-106 & 584 & Calcium & 41600 & & & & UG/L & 06/10/2009 & & HCJ-161-09 \\
\hline GWA04801VL & USGS-106 & 584 & Carbon disulfide & 5 & & U & & UG/L & 06/10/2009 & & HCJ-148-09 \\
\hline GWA04802VL & USGS-106 & 584 & Carbon disulfide & 5 & & $u$ & & UG/L & 06/10/2009 & & HCJ-148-09 \\
\hline GWA04801VL & USGS-106 & 584 & Carbon tetrachloride & 1 & & $u$ & & UG/L & $06 / 10 / 2009$ & & HCJ-148-09 \\
\hline GWA04802VL & USGS-106 & 584 & Carbon tetrachloride & 1 & & U & & UG/L & 06/10/2009 & & HCJ-148-09 \\
\hline GWA04801AN & USGS-106 & 584 & Chloride & 14.9 & & & & $M G / L$ & $06 / 10 / 2009$ & & HCJ-159-09 \\
\hline GWA04802AN & USGS-106 & 584 & Chloride & 15 & & & & MG/L & 06/10/2009 & & HCJ-159-09 \\
\hline GWA04801VL & USGS-106 & 584 & Chlorobenzene & 1 & & $U$ & & UG/L & $06 / 10 / 2009$ & & HCJ-148-09 \\
\hline GWA04802VL & USGS-106 & 584 & Chlorobenzene & 1 & & U & & UG/L & $06 / 10 / 2009$ & & HCJ-148-09 \\
\hline GWA04801VL & USGS-106 & 584 & Chloroethane & 1 & & $u$ & & UG/L & $06 / 10 / 2009$ & & HCJ-148-09 \\
\hline GWA04802VL & USGS-106 & 584 & Chloroethane & 1 & & $u$ & & UG/L & 06/10/2009 & & HCJ-148-09 \\
\hline GWA04801VL & USGS-106 & 584 & Chloroform & 1 & & U & & UG/L & $06 / 10 / 2009$ & & HCJ-148-09 \\
\hline GWA04802VL & USGS-106 & 584 & Chloroform & 1 & & $U$ & & UG/L & $06 / 10 / 2009$ & & HCJ-148-09 \\
\hline GWA04801VL & USGS-106 & 584 & Chloromethane & 1 & & $u$ & & UG/L & 06/10/2009 & & HCJ-148-09 \\
\hline GWA04802VL & USGS-106 & 584 & Chloromethane & 1 & & $U$ & & UG/L & $06 / 10 / 2009$ & & HCJ-148-09 \\
\hline GWA048012X & USGS-106 & 584 & Chromium & 6.93 & & & & UG/L & $06 / 10 / 2009$ & & HCJ-161-09 \\
\hline GWA048022X & USGS-106 & 584 & Chromium & 6.85 & & & & UG/L & 06/10/2009 & & HCJ-161-09 \\
\hline GWA04801VL & USGS-106 & 584 & cis-1,2-Dichloroethylene & 1 & & $u$ & & UG/L & $06 / 10 / 2009$ & & HCJ-148-09 \\
\hline GWA04802VL & USGS-106 & 584 & cis-1,2-Dichloroethylene & 1 & & $\mathrm{U}$ & & UG/L & $06 / 10 / 2009$ & & HCJ-148-09 \\
\hline GWA04801VL & USGS-106 & 584 & cis-1,3-Dichloropropylene & 1 & & U & & UG/L & $06 / 10 / 2009$ & & HCJ-148-09 \\
\hline GWA04802VL & USGS-106 & 584 & cis-1,3-Dichloropropylene & 1 & & $u$ & & UG/L & 06/10/2009 & & HCJ-148-09 \\
\hline GWA048012X & USGS-106 & 584 & Cobalt & 4.33 & & B & & UG/L & 06/10/2009 & & HCJ-161-09 \\
\hline GWA048022X & USGS-106 & 584 & Cobalt & 0.229 & & B & & UG/L & $06 / 10 / 2009$ & & HCJ-161-09 \\
\hline GWA048012X & USGS-106 & 584 & Copper & 1.03 & & B & & UG/L & 06/10/2009 & & HCJ-161-09 \\
\hline GWA048022X & USGS-106 & 584 & Copper & 1.02 & & B & & UG/L & 06/10/2009 & & HCJ-161-09 \\
\hline GWA04801VL & USGS-106 & 584 & Cyclohexane & 1 & & $\mathrm{U}$ & & UG/L & 06/10/2009 & & HCJ-148-09 \\
\hline GWA04802VL & USGS-106 & 584 & Cyclohexane & 1 & & U & & UG/L & $06 / 10 / 2009$ & & HCJ-148-09 \\
\hline GWA04801VL & USGS-106 & 584 & Dibromochloromethane & 1 & & U & & UG/L & 06/10/2009 & & HCJ-148-09 \\
\hline GWA04802VL & USGS-106 & 584 & Dibromochloromethane & 1 & & $u$ & & UG/L & 06/10/2009 & & HCJ-148-09 \\
\hline GWA04801VL & USGS-106 & 584 & Dichlorodifluoromethane & 1 & & $u$ & & UG/L & $06 / 10 / 2009$ & & HCJ-148-09 \\
\hline GWA04802VL & USGS-106 & 584 & Dichlorodifluoromethane & 1 & & U & & UG/L & $06 / 10 / 2009$ & & HCJ-148-09 \\
\hline GWA04801VL & USGS-106 & 584 & Ethylbenzene & 1 & & $u$ & & UG/L & 06/10/2009 & & HCJ-148-09 \\
\hline GWA04802VL & USGS-106 & 584 & Ethylbenzene & 1 & & U & & UG/L & 06/10/2009 & & HCJ-148-09 \\
\hline GWA04801AN & USGS-106 & 584 & Fluoride & 0.211 & & $\mathrm{~J}$ & & $M G / L$ & $06 / 10 / 2009$ & & HCJ-159-09 \\
\hline GWA04802AN & USGS-106 & 584 & Fluoride & 0.192 & & $\mathrm{~J}$ & & $M G / L$ & $06 / 10 / 2009$ & & HCJ-159-09 \\
\hline GWA04801RH & USGS-106 & 584 & Gross Alpha & $1.88 \mathrm{E}+00$ & 7.57E-01 & & UJ & $\mathrm{PCl} / \mathrm{L}$ & 06/10/2009 & $1.94 \mathrm{E}+00$ & BAM-120-09 \\
\hline GWA04802RH & USGS-106 & 584 & Gross Alpha & $2.39 E+00$ & 8.20E-01 & & $\mathrm{J}$ & $\mathrm{PCl} / \mathrm{L}$ & 06/10/2009 & $1.88 \mathrm{E}+00$ & BAM-120-09 \\
\hline GWA04801RH & USGS-106 & 584 & Gross Beta & $3.42 \mathrm{E}+00$ & 8.76E-01 & & & $\mathrm{PCI} / \mathrm{L}$ & 06/10/2009 & $2.30 \mathrm{E}+00$ & BAM-120-09 \\
\hline GWA04802RH & USGS-106 & 584 & Gross Beta & $2.59 \mathrm{E}+00$ & 7.53E-01 & & & $\mathrm{PCl} / \mathrm{L}$ & 06/10/2009 & $2.03 \mathrm{E}+00$ & BAM-120-09 \\
\hline GWA04801UX & USGS-106 & 584 & lodine-129 & $5.00 \mathrm{E}-02$ & 2.69E-02 & & U & $\mathrm{PCl} / \mathrm{L}$ & 06/10/2009 & $9.95 \mathrm{E}-02$ & BAM-118-09 \\
\hline GWA048012X & USGS-106 & 584 & Iron & 184 & & & & UG/L & $06 / 10 / 2009$ & & HCJ-161-09 \\
\hline
\end{tabular}


Table A-2. (continued).

\begin{tabular}{|c|c|c|c|c|c|c|c|c|c|c|c|}
\hline $\begin{array}{c}\text { Field Sample } \\
\text { Number }\end{array}$ & Location & Depth & Compound & $\begin{array}{c}\text { Sample } \\
\text { Result }\end{array}$ & $\begin{array}{c}\text { Sample } \\
\text { Error }\end{array}$ & $\begin{array}{c}\text { Result } \\
\text { Qualifier }\end{array}$ & $\begin{array}{c}\text { Validation } \\
\text { Flag }\end{array}$ & $\begin{array}{c}\text { Sample } \\
\text { Units }\end{array}$ & $\begin{array}{c}\text { Date Sample } \\
\text { Collected }\end{array}$ & MDA & L\&V Report Number \\
\hline GWA048022X & USGS-106 & 584 & Iron & 177 & & & & UG/L & $06 / 10 / 2009$ & & HCJ-161-09 \\
\hline GWA04801VL & USGS-106 & 584 & Isopropylbenzene & 1 & & U & & UG/L & 06/10/2009 & & HCJ-148-09 \\
\hline GWA04802VL & USGS-106 & 584 & Isopropylbenzene & 1 & & $u$ & & UG/L & 06/10/2009 & & HCJ-148-09 \\
\hline GWA048012X & USGS-106 & 584 & Lead & 13.2 & & & & UG/L & 06/10/2009 & & HCJ-161-09 \\
\hline GWA048022X & USGS-106 & 584 & Lead & 13.3 & & & & UG/L & 06/10/2009 & & HCJ-161-09 \\
\hline GWA048012X & USGS-106 & 584 & Magnesium & 17100 & & & & UG/L & 06/10/2009 & & HCJ-161-09 \\
\hline GWA048022X & USGS-106 & 584 & Magnesium & 16300 & & & & UG/L & $06 / 10 / 2009$ & & HCJ-161-09 \\
\hline GWA048012X & USGS-106 & 584 & Manganese & 7.12 & & B & & UG/L & 06/10/2009 & & HCJ-161-09 \\
\hline GWA048022X & USGS-106 & 584 & Manganese & 1 & & U & & UG/L & $06 / 10 / 2009$ & & HCJ-161-09 \\
\hline GWA048012X & USGS-106 & 584 & Mercury & 0.067 & & $u$ & & UG/L & 06/10/2009 & & HCJ-161-09 \\
\hline GWA048022X & USGS-106 & 584 & Mercury & 0.067 & & $U$ & & UG/L & 06/10/2009 & & HCJ-161-09 \\
\hline GWA04801VL & USGS-106 & 584 & Methyl acetate & 5 & & U & & UG/L & $06 / 10 / 2009$ & & HCJ-148-09 \\
\hline GWA04802VL & USGS-106 & 584 & Methyl acetate & 5 & & $U$ & & UG/L & $06 / 10 / 2009$ & & HCJ-148-09 \\
\hline GWA04801VL & USGS-106 & 584 & Methyl cyclohexane & 1 & & $U$ & & UG/L & 06/10/2009 & & HCJ-148-09 \\
\hline GWA04802VL & USGS-106 & 584 & Methyl cyclohexane & 1 & & U & & UG/L & 06/10/2009 & & HCJ-148-09 \\
\hline GWA04801VL & USGS-106 & 584 & Methylene Chloride & 5 & & $U$ & & UG/L & $06 / 10 / 2009$ & & HCJ-148-09 \\
\hline GWA04802VL & USGS-106 & 584 & Methylene Chloride & 5 & & $U$ & & UG/L & 06/10/2009 & & HCJ-148-09 \\
\hline GWA048012X & USGS-106 & 584 & Nickel & 1.43 & & B & & UG/L & $06 / 10 / 2009$ & & HCJ-161-09 \\
\hline GWA048022X & USGS-106 & 584 & Nickel & 0.984 & & B & & UG/L & 06/10/2009 & & HCJ-161-09 \\
\hline GWA04801N2 & USGS-106 & 584 & Nitrogen, Nitrate/Nitrite & 1.04 & & & & $M G / L$ & $06 / 10 / 2009$ & & HCJ-155-09 \\
\hline GWA04802N2 & USGS-106 & 584 & Nitrogen, Nitrate/Nitrite & 1.04 & & & & MG/L & 06/10/2009 & & HCJ-155-09 \\
\hline GWA048012X & USGS-106 & 584 & Potassium & 2150 & & B & & UG/L & $06 / 10 / 2009$ & & HCJ-161-09 \\
\hline GWA048022X & USGS-106 & 584 & Potassium & 2000 & & B & & UG/L & $06 / 10 / 2009$ & & HCJ-161-09 \\
\hline GWA048012X & USGS-106 & 584 & Selenium & 1.11 & & B & & UG/L & 06/10/2009 & & HCJ-161-09 \\
\hline GWA048022X & USGS-106 & 584 & Selenium & 1 & & $U$ & & UG/L & 06/10/2009 & & HCJ-161-09 \\
\hline GWA048012X & USGS-106 & 584 & Silver & 0.2 & & U & & UG/L & $06 / 10 / 2009$ & & HCJ-161-09 \\
\hline GWA048022X & USGS-106 & 584 & Silver & 0.2 & & $U$ & & UG/L & 06/10/2009 & & HCJ-161-09 \\
\hline GWA048012X & USGS-106 & 584 & Sodium & 7160 & & & & UG/L & 06/10/2009 & & HCJ-161-09 \\
\hline GWA048022X & USGS-106 & 584 & Sodium & 7220 & & & & UG/L & $06 / 10 / 2009$ & & HCJ-161-09 \\
\hline GWA048012X & USGS-106 & 584 & Strontium & 226 & & & & UG/L & $06 / 10 / 2009$ & & HCJ-161-09 \\
\hline GWA048022X & USGS-106 & 584 & Strontium & 226 & & & & UG/L & 06/10/2009 & & HCJ-161-09 \\
\hline GWA04801RH & USGS-106 & 584 & Strontium-90 & $-6.90 \mathrm{E}-02$ & $9.60 \mathrm{E}-02$ & & $U$ & $\mathrm{PCl} / \mathrm{L}$ & 06/10/2009 & 3.65E-01 & BAM-120-09 \\
\hline GWA04802RH & USGS-106 & 584 & Strontium-90 & $-3.19 \mathrm{E}-04$ & 7.13E-02 & & $U$ & $\mathrm{PCl} / \mathrm{L}$ & 06/10/2009 & $2.58 \mathrm{E}-01$ & BAM-120-09 \\
\hline GWA04801VL & USGS-106 & 584 & Styrene & 1 & & U & & UG/L & $06 / 10 / 2009$ & & HCJ-148-09 \\
\hline GWA04802VL & USGS-106 & 584 & Styrene & 1 & & $U$ & & UG/L & 06/10/2009 & & HCJ-148-09 \\
\hline GWA04801AN & USGS-106 & 584 & Sulfate & 23.1 & & & & $M G / L$ & $06 / 10 / 2009$ & & HCJ-159-09 \\
\hline GWA04802AN & USGS-106 & 584 & Sulfate & 23.1 & & & & MG/L & $06 / 10 / 2009$ & & HCJ-159-09 \\
\hline GWA04801RH & USGS-106 & 584 & Technetium-99 & $-3.06 \mathrm{E}+00$ & $1.58 \mathrm{E}+00$ & & U & $\mathrm{PCl} / \mathrm{L}$ & 06/10/2009 & $5.85 \mathrm{E}+00$ & BAM-120-09 \\
\hline GWA04802RH & USGS-106 & 584 & Technetium-99 & $-2.40 \mathrm{E}+00$ & $1.59 \mathrm{E}+00$ & & $u$ & $\mathrm{PCl} / \mathrm{L}$ & 06/10/2009 & $5.78 \mathrm{E}+00$ & BAM-120-09 \\
\hline GWA04801VL & USGS-106 & 584 & Tert-butyl methyl ether & 1 & & $U$ & & UG/L & 06/10/2009 & & HCJ-148-09 \\
\hline GWA04802VL & USGS-106 & 584 & Tert-butyl methyl ether & 1 & & U & & UG/L & 06/10/2009 & & HCJ-148-09 \\
\hline GWA04801VL & USGS-106 & 584 & Tetrachloroethylene & 1 & & $U$ & & UG/L & $06 / 10 / 2009$ & & HCJ-148-09 \\
\hline GWA04802VL & USGS-106 & 584 & Tetrachloroethylene & 1 & & $\mathrm{U}$ & & UG/L & 06/10/2009 & & HCJ-148-09 \\
\hline GWA048012X & USGS-106 & 584 & Thallium & 0.3 & & $\mathrm{U}$ & & UG/L & $06 / 10 / 2009$ & & HCJ-161-09 \\
\hline
\end{tabular}


Table A-2. (continued).

\begin{tabular}{|c|c|c|c|c|c|c|c|c|c|c|c|}
\hline $\begin{array}{c}\text { Field Sample } \\
\text { Number }\end{array}$ & Location & Depth & Compound & $\begin{array}{c}\text { Sample } \\
\text { Result }\end{array}$ & $\begin{array}{l}\text { Sample } \\
\text { Error }\end{array}$ & $\begin{array}{c}\text { Result } \\
\text { Qualifier } \\
\end{array}$ & $\begin{array}{c}\text { Validation } \\
\text { Flag }\end{array}$ & $\begin{array}{c}\text { Sample } \\
\text { Units }\end{array}$ & $\begin{array}{c}\text { Date Sample } \\
\text { Collected }\end{array}$ & MDA & L\&V Report Number \\
\hline GWA048022X & USGS-106 & 584 & Thallium & 0.3 & & $U$ & & UG/L & $06 / 10 / 2009$ & & HCJ-161-09 \\
\hline GWA04801VL & USGS-106 & 584 & Toluene & 1 & & U & & UG/L & 06/10/2009 & & HCJ-148-09 \\
\hline GWA04802VL & USGS-106 & 584 & Toluene & 1 & & $\mathrm{u}$ & & UG/L & $06 / 10 / 2009$ & & HCJ-148-09 \\
\hline GWA04801VL & USGS-106 & 584 & trans-1,2-Dichloroethylene & 1 & & $\mathrm{u}$ & & UG/L & $06 / 10 / 2009$ & & HCJ-148-09 \\
\hline GWA04802VL & USGS-106 & 584 & trans-1,2-Dichloroethylene & 1 & & U & & UG/L & $06 / 10 / 2009$ & & HCJ-148-09 \\
\hline GWA04801VL & USGS-106 & 584 & trans-1,3-Dichloropropylene & 1 & & $\mathrm{u}$ & & UG/L & $06 / 10 / 2009$ & & HCJ-148-09 \\
\hline GWA04802VL & USGS-106 & 584 & trans-1,3-Dichloropropylene & 1 & & $\mathrm{U}$ & & UG/L & $06 / 10 / 2009$ & & HCJ-148-09 \\
\hline GWA04801VL & USGS-106 & 584 & Trichloroethylene & 1 & & U & & UG/L & $06 / 10 / 2009$ & & HCJ-148-09 \\
\hline GWA04802VL & USGS-106 & 584 & Trichloroethylene & 1 & & U & & UG/L & $06 / 10 / 2009$ & & HCJ-148-09 \\
\hline GWA04801VL & USGS-106 & 584 & Trichlorofluoromethane & 1 & & $\mathrm{u}$ & & UG/L & $06 / 10 / 2009$ & & HCJ-148-09 \\
\hline GWA04802VL & USGS-106 & 584 & Trichlorofluoromethane & 1 & & $\mathrm{U}$ & & UG/L & $06 / 10 / 2009$ & & HCJ-148-09 \\
\hline GWA04801VL & USGS-106 & 584 & Trichlorotrifluoroethane & 5 & & $U$ & & UG/L & $06 / 10 / 2009$ & & HCJ-148-09 \\
\hline GWA04802VL & USGS-106 & 584 & Trichlorotrifluoroethane & 5 & & $u$ & & UG/L & $06 / 10 / 2009$ & & HCJ-148-09 \\
\hline GWA04801R8 & USGS-106 & 584 & Tritium & $4.67 \mathrm{E}+02$ & $1.11 \mathrm{E}+02$ & & & $\mathrm{PCl} / \mathrm{L}$ & $06 / 10 / 2009$ & $3.05 E+02$ & BAM-119-09 \\
\hline GWA04802R8 & USGS-106 & 584 & Tritium & $4.35 \mathrm{E}+02$ & $1.09 \mathrm{E}+02$ & & & $\mathrm{PCl} / \mathrm{L}$ & $06 / 10 / 2009$ & $3.05 \mathrm{E}+02$ & BAM-119-09 \\
\hline GWA048012X & USGS-106 & 584 & Uranium & 1.7 & & B & & UG/L & 06/10/2009 & & HCJ-161-09 \\
\hline GWA048022X & USGS-106 & 584 & Uranium & 1.64 & & B & & UG/L & $06 / 10 / 2009$ & & HCJ-161-09 \\
\hline GWA048012X & USGS-106 & 584 & Vanadium & 3 & & $\mathrm{U}$ & & UG/L & $06 / 10 / 2009$ & & HCJ-161-09 \\
\hline GWA048022X & USGS-106 & 584 & Vanadium & 3 & & U & & UG/L & 06/10/2009 & & HCJ-161-09 \\
\hline GWA04801VL & USGS-106 & 584 & Vinyl Chloride & 1 & & $u$ & & UG/L & $06 / 10 / 2009$ & & HCJ-148-09 \\
\hline GWA04802VL & USGS-106 & 584 & Vinyl Chloride & 1 & & $\mathrm{U}$ & & UG/L & $06 / 10 / 2009$ & & HCJ-148-09 \\
\hline GWA04801VL & USGS-106 & 584 & Xylene (Total) & 1 & & U & & UG/L & 06/10/2009 & & HCJ-148-09 \\
\hline GWA04802VL & USGS-106 & 584 & Xylene (Total) & 1 & & U & & UG/L & $06 / 10 / 2009$ & & HCJ-148-09 \\
\hline GWA048012X & USGS-106 & 584 & Zinc & 161 & & & & UG/L & $06 / 10 / 2009$ & & HCJ-161-09 \\
\hline GWA048022X & USGS-106 & 584 & Zinc & 165 & & & & UG/L & $06 / 10 / 2009$ & & HCJ-161-09 \\
\hline GWA04802UX & USGS-106 & 584 & lodine-129 & $-4.01 \mathrm{E}-02$ & $4.54 \mathrm{E}-02$ & & $U$ & $\mathrm{PCI} / \mathrm{L}$ & $06 / 10 / 2009$ & 1.23E-01 & BAM-118-09 \\
\hline GWA049012X & USGS-107 & 477 & Aluminum & 15 & & U & & UG/L & 06/10/2009 & & HCJ-161-09 \\
\hline GWA049012X & USGS-107 & 477 & Antimony & 1 & & U & & UG/L & $06 / 10 / 2009$ & & HCJ-161-09 \\
\hline GWA049012X & USGS-107 & 477 & Arsenic & 1.6 & & U & & UG/L & $06 / 10 / 2009$ & & HCJ-161-09 \\
\hline GWA049012X & USGS-107 & 477 & Barium & 56 & & B & & UG/L & $06 / 10 / 2009$ & & HCJ-161-09 \\
\hline GWA049012X & USGS-107 & 477 & Beryllium & 0.1 & & $U$ & & UG/L & 06/10/2009 & & HCJ-161-09 \\
\hline GWA049012X & USGS-107 & 477 & Cadmium & 0.11 & & U & & UG/L & $06 / 10 / 2009$ & & HCJ-161-09 \\
\hline GWA049012X & USGS-107 & 477 & Calcium & 33900 & & & & UG/L & $06 / 10 / 2009$ & & HCJ-161-09 \\
\hline GWA049012X & USGS-107 & 477 & Chromium & 4.04 & & B & & UG/L & $06 / 10 / 2009$ & & HCJ-161-09 \\
\hline GWA049012X & USGS-107 & 477 & Cobalt & 3.88 & & B & & UG/L & $06 / 10 / 2009$ & & HCJ-161-09 \\
\hline GWA049012X & USGS-107 & 477 & Copper & 0.681 & & B & & UG/L & $06 / 10 / 2009$ & & HCJ-161-09 \\
\hline GWA049012X & USGS-107 & 477 & Iron & 141 & & & & UG/L & $06 / 10 / 2009$ & & HCJ-161-09 \\
\hline GWA049012X & USGS-107 & 477 & Lead & 0.5 & & 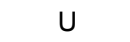 & & UG/L & $06 / 10 / 2009$ & & HCJ-161-09 \\
\hline GWA049012X & USGS-107 & 477 & Magnesium & 15900 & & & & UG/L & $06 / 10 / 2009$ & & HCJ-161-09 \\
\hline GWA049012X & USGS-107 & 477 & Manganese & 5.91 & & B & & UG/L & $06 / 10 / 2009$ & & HCJ-161-09 \\
\hline GWA049012X & USGS-107 & 477 & Mercury & 0.067 & & U & & UG/L & $06 / 10 / 2009$ & & HCJ-161-09 \\
\hline GWA049012X & USGS-107 & 477 & Nickel & 1.04 & & B & & UG/L & $06 / 10 / 2009$ & & HCJ-161-09 \\
\hline GWA049012X & USGS-107 & 477 & Potassium & 2980 & & B & & UG/L & $06 / 10 / 2009$ & & HCJ-161-09 \\
\hline GWA049012X & USGS-107 & 477 & Selenium & 1.27 & & B & & UG/L & $06 / 10 / 2009$ & & HCJ-161-09 \\
\hline
\end{tabular}


Table A-2. (continued).

\begin{tabular}{|c|c|c|c|c|c|c|c|c|c|c|c|}
\hline $\begin{array}{c}\text { Field Sample } \\
\text { Number }\end{array}$ & Location & Depth & Compound & $\begin{array}{c}\text { Sample } \\
\text { Result }\end{array}$ & $\begin{array}{c}\text { Sample } \\
\text { Error }\end{array}$ & $\begin{array}{c}\text { Result } \\
\text { Qualifier }\end{array}$ & $\begin{array}{c}\text { Validation } \\
\text { Flag }\end{array}$ & $\begin{array}{c}\text { Sample } \\
\text { Units }\end{array}$ & $\begin{array}{c}\text { Date Sample } \\
\text { Collected }\end{array}$ & MDA & L\&V Report Number \\
\hline GWA049012X & USGS-107 & 477 & Silver & 0.2 & & $U$ & & UG/L & 06/10/2009 & & HCJ-161-09 \\
\hline GWA049012X & USGS-107 & 477 & Sodium & 16200 & & & & UG/L & 06/10/2009 & & HCJ-161-09 \\
\hline GWA049012X & USGS-107 & 477 & Strontium & 200 & & & & UG/L & 06/10/2009 & & HCJ-161-09 \\
\hline GWA049012X & USGS-107 & 477 & Thallium & 0.3 & & $\mathrm{U}$ & & UG/L & 06/10/2009 & & HCJ-161-09 \\
\hline GWA049012X & USGS-107 & 477 & Uranium & 2.7 & & B & & UG/L & 06/10/2009 & & HCJ-161-09 \\
\hline GWA049012X & USGS-107 & 477 & Vanadium & 3.33 & & B & & UG/L & 06/10/2009 & & HCJ-161-09 \\
\hline GWA049012X & USGS-107 & 477 & Zinc & 3 & & $\mathrm{U}$ & & UG/L & 06/10/2009 & & HCJ-161-09 \\
\hline GWA04901A1 & USGS-107 & 477 & Alkalinity, Total as $\mathrm{CaCO} 3$ & 144 & & & & $M G / L$ & 06/10/2009 & & HCJ-157-09 \\
\hline GWA04901AN & USGS-107 & 477 & Bromide & 0 & & U & & $M G / L$ & 06/10/2009 & & HCJ-159-09 \\
\hline GWA04901AN & USGS-107 & 477 & Chloride & 21.2 & & & & $M G / L$ & 06/10/2009 & & HCJ-159-09 \\
\hline GWA04901AN & USGS-107 & 477 & Fluoride & 0.423 & & $\mathrm{~J}$ & & $M G / L$ & 06/10/2009 & & HCJ-159-09 \\
\hline GWA04901AN & USGS-107 & 477 & Sulfate & 26.6 & & & & $M G / L$ & 06/10/2009 & & HCJ-159-09 \\
\hline GWA04901N2 & USGS-107 & 477 & Nitrogen, Nitrate/Nitrite & 1.23 & & & & $M G / L$ & 06/10/2009 & & HCJ-155-09 \\
\hline GWA04901VL & USGS-107 & 477 & Dichlorodifluoromethane & 1 & & $\mathrm{U}$ & & UG/L & 06/10/2009 & & HCJ-148-09 \\
\hline GWA04901VL & USGS-107 & 477 & Chloromethane & 1 & & U & & UG/L & 06/10/2009 & & HCJ-148-09 \\
\hline GWA04901VL & USGS-107 & 477 & Vinyl Chloride & 1 & & U & & UG/L & 06/10/2009 & & HCJ-148-09 \\
\hline GWA04901VL & USGS-107 & 477 & Bromomethane & 1 & & $U$ & & UG/L & 06/10/2009 & & HCJ-148-09 \\
\hline GWA04901VL & USGS-107 & 477 & Chloroethane & 1 & & $\mathrm{U}$ & & UG/L & 06/10/2009 & & HCJ-148-09 \\
\hline GWA04901VL & USGS-107 & 477 & Trichlorofluoromethane & 1 & & U & & UG/L & 06/10/2009 & & HCJ-148-09 \\
\hline GWA04901VL & USGS-107 & 477 & Acetone & 5 & & $u$ & & UG/L & 06/10/2009 & & HCJ-148-09 \\
\hline GWA04901VL & USGS-107 & 477 & 1,1-Dichloroethene & 1 & & $\mathrm{U}$ & & UG/L & 06/10/2009 & & HCJ-148-09 \\
\hline GWA04901VL & USGS-107 & 477 & Methyl acetate & 5 & & U & & UG/L & 06/10/2009 & & HCJ-148-09 \\
\hline GWA04901VL & USGS-107 & 477 & Methylene Chloride & 5 & & U & & UG/L & 06/10/2009 & & HCJ-148-09 \\
\hline GWA04901VL & USGS-107 & 477 & Carbon disulfide & 5 & & $U$ & & UG/L & 06/10/2009 & & HCJ-148-09 \\
\hline GWA04901VL & USGS-107 & 477 & Tert-butyl methyl ether & 1 & & $\mathrm{U}$ & & UG/L & 06/10/2009 & & HCJ-148-09 \\
\hline GWA04901VL & USGS-107 & 477 & trans-1,2-Dichloroethylene & 1 & & U & & UG/L & 06/10/2009 & & HCJ-148-09 \\
\hline GWA04901VL & USGS-107 & 477 & 1,1-Dichloroethane & 1 & & $\mathrm{U}$ & & UG/L & 06/10/2009 & & HCJ-148-09 \\
\hline GWA04901VL & USGS-107 & 477 & 2-Butanone & 5 & & $\mathrm{U}$ & & UG/L & 06/10/2009 & & HCJ-148-09 \\
\hline GWA04901VL & USGS-107 & 477 & cis-1,2-Dichloroethylene & 1 & & U & & UG/L & 06/10/2009 & & HCJ-148-09 \\
\hline GWA04901VL & USGS-107 & 477 & Chloroform & 1 & & U & & UG/L & 06/10/2009 & & HCJ-148-09 \\
\hline GWA04901VL & USGS-107 & 477 & 1,1,1-Trichloroethane & 1 & & $u$ & & UG/L & 06/10/2009 & & HCJ-148-09 \\
\hline GWA04901VL & USGS-107 & 477 & Cyclohexane & 1 & & $\mathrm{U}$ & & UG/L & 06/10/2009 & & HCJ-148-09 \\
\hline GWA04901VL & USGS-107 & 477 & Carbon tetrachloride & 1 & & U & & UG/L & 06/10/2009 & & HCJ-148-09 \\
\hline GWA04901VL & USGS-107 & 477 & 1,2-Dichloroethane & 1 & & $u$ & & UG/L & 06/10/2009 & & HCJ-148-09 \\
\hline GWA04901VL & USGS-107 & 477 & Benzene & 1 & & $\mathrm{u}$ & & UG/L & 06/10/2009 & & HCJ-148-09 \\
\hline GWA04901VL & USGS-107 & 477 & Trichloroethylene & 1 & & U & & UG/L & 06/10/2009 & & HCJ-148-09 \\
\hline GWA04901VL & USGS-107 & 477 & 1,2-Dichloropropane & 1 & & U & & UG/L & 06/10/2009 & & HCJ-148-09 \\
\hline GWA04901VL & USGS-107 & 477 & Methyl cyclohexane & 1 & & $u$ & & UG/L & 06/10/2009 & & HCJ-148-09 \\
\hline GWA04901VL & USGS-107 & 477 & Bromodichloromethane & 1 & & $\mathrm{U}$ & & UG/L & 06/10/2009 & & HCJ-148-09 \\
\hline GWA04901VL & USGS-107 & 477 & 4-Methyl-2-pentanone & 5 & & $\mathrm{U}$ & & UG/L & 06/10/2009 & & HCJ-148-09 \\
\hline GWA04901VL & USGS-107 & 477 & cis-1,3-Dichloropropylene & 1 & & $\mathrm{U}$ & & UG/L & 06/10/2009 & & HCJ-148-09 \\
\hline GWA04901VL & USGS-107 & 477 & Toluene & 1 & & $\mathrm{U}$ & & UG/L & 06/10/2009 & & HCJ-148-09 \\
\hline GWA04901VL & USGS-107 & 477 & trans-1,3-Dichloropropylene & 1 & & $u$ & & UG/L & 06/10/2009 & & HCJ-148-09 \\
\hline GWA04901VL & USGS-107 & 477 & 1,1,2-Trichloroethane & 1 & & $U$ & & UG/L & 06/10/2009 & & HCJ-148-09 \\
\hline
\end{tabular}


Table A-2. (continued).

\begin{tabular}{|c|c|c|c|c|c|c|c|c|c|c|c|}
\hline $\begin{array}{c}\text { Field Sample } \\
\text { Number }\end{array}$ & Location & Depth & Compound & $\begin{array}{c}\text { Sample } \\
\text { Result }\end{array}$ & $\begin{array}{l}\text { Sample } \\
\text { Error }\end{array}$ & $\begin{array}{c}\text { Result } \\
\text { Qualifier }\end{array}$ & $\begin{array}{c}\text { Validation } \\
\text { Flag }\end{array}$ & $\begin{array}{c}\text { Sample } \\
\text { Units }\end{array}$ & $\begin{array}{c}\text { Date Sample } \\
\text { Collected }\end{array}$ & MDA & L\&V Report Number \\
\hline GWA04901VL & USGS-107 & 477 & 2-Hexanone & 5 & & $U$ & & UG/L & $06 / 10 / 2009$ & & HCJ-148-09 \\
\hline GWA04901VL & USGS-107 & 477 & Tetrachloroethylene & 1 & & U & & UG/L & 06/10/2009 & & HCJ-148-09 \\
\hline GWA04901VL & USGS-107 & 477 & Dibromochloromethane & 1 & & $\mathrm{U}$ & & UG/L & $06 / 10 / 2009$ & & HCJ-148-09 \\
\hline GWA04901VL & USGS-107 & 477 & 1,2-Dibromoethane & 1 & & $\mathrm{U}$ & & UG/L & $06 / 10 / 2009$ & & HCJ-148-09 \\
\hline GWA04901VL & USGS-107 & 477 & Chlorobenzene & 1 & & U & & UG/L & $06 / 10 / 2009$ & & HCJ-148-09 \\
\hline GWA04901VL & USGS-107 & 477 & Ethylbenzene & 1 & & $\mathrm{u}$ & & UG/L & $06 / 10 / 2009$ & & HCJ-148-09 \\
\hline GWA04901VL & USGS-107 & 477 & Styrene & 1 & & $\mathrm{U}$ & & UG/L & $06 / 10 / 2009$ & & HCJ-148-09 \\
\hline GWA04901VL & USGS-107 & 477 & Bromoform & 1 & & U & & UG/L & $06 / 10 / 2009$ & & HCJ-148-09 \\
\hline GWA04901VL & USGS-107 & 477 & 1,1,2,2-Tetrachloroethane & 1 & & U & & UG/L & 06/10/2009 & & HCJ-148-09 \\
\hline GWA04901VL & USGS-107 & 477 & Isopropylbenzene & 1 & & $\mathrm{U}$ & & UG/L & $06 / 10 / 2009$ & & HCJ-148-09 \\
\hline GWA04901VL & USGS-107 & 477 & 1,3-Dichlorobenzene & 1 & & $\mathrm{U}$ & & UG/L & $06 / 10 / 2009$ & & HCJ-148-09 \\
\hline GWA04901VL & USGS-107 & 477 & 1,4-Dichlorobenzene & 1 & & U & & UG/L & $06 / 10 / 2009$ & & HCJ-148-09 \\
\hline GWA04901VL & USGS-107 & 477 & 1,2-Dibromo-3-chloropropane & 1 & & U & & UG/L & $06 / 10 / 2009$ & & HCJ-148-09 \\
\hline GWA04901VL & USGS-107 & 477 & Trichlorotrifluoroethane & 5 & & $\mathrm{u}$ & & UG/L & $06 / 10 / 2009$ & & HCJ-148-09 \\
\hline GWA04901VL & USGS-107 & 477 & Xylene (Total) & 1 & & U & & UG/L & $06 / 10 / 2009$ & & HCJ-148-09 \\
\hline GWA04901VL & USGS-107 & 477 & 1,2,4-Trichlorobenzene & 1 & & U & & UG/L & $06 / 10 / 2009$ & & HCJ-148-09 \\
\hline GWA04901VL & USGS-107 & 477 & 1,2-Dichlorobenzene & 1 & & U & & UG/L & 06/10/2009 & & HCJ-148-09 \\
\hline GWA04901R8 & USGS-107 & 477 & Tritium & $0.00 \mathrm{E}+00$ & $8.77 E+01$ & & $\mathrm{U}$ & $\mathrm{PCl} / \mathrm{L}$ & $06 / 10 / 2009$ & $3.05 E+02$ & BAM-119-09 \\
\hline GWA04901RH & USGS-107 & 477 & Gross Alpha & $3.14 \mathrm{E}+00$ & $9.85 \mathrm{E}-01$ & & & $\mathrm{PCl} / \mathrm{L}$ & $06 / 10 / 2009$ & $2.30 \mathrm{E}+00$ & BAM-120-09 \\
\hline GWA04901RH & USGS-107 & 477 & Gross Beta & $3.04 \mathrm{E}+00$ & $1.06 \mathrm{E}+00$ & & UJ & $\mathrm{PCI} / \mathrm{L}$ & $06 / 10 / 2009$ & $3.12 \mathrm{E}+00$ & BAM-120-09 \\
\hline GWA04901RH & USGS-107 & 477 & Strontium-90 & 1.49E-01 & $1.06 \mathrm{E}-01$ & & $\mathrm{U}$ & $\mathrm{PCl} / \mathrm{L}$ & $06 / 10 / 2009$ & 3.53E-01 & BAM-120-09 \\
\hline GWA04901RH & USGS-107 & 477 & Technetium-99 & $-1.42 \mathrm{E}+00$ & $1.74 \mathrm{E}+00$ & & $\mathrm{U}$ & $\mathrm{PCl} / \mathrm{L}$ & $06 / 10 / 2009$ & $6.19 \mathrm{E}+00$ & BAM-120-09 \\
\hline GWA04901UX & USGS-107 & 477 & lodine-129 & 6.77E-03 & $2.47 \mathrm{E}-02$ & & U & $\mathrm{PCl} / \mathrm{L}$ & $06 / 10 / 2009$ & 8.42E-02 & BAM-118-09 \\
\hline GWA042012X & USGS-109 & 621 & Aluminum & 15 & & U & & UG/L & 06/10/2009 & & HCJ-161-09 \\
\hline GWA042012X & USGS-109 & 621 & Antimony & 1 & & U & & UG/L & $06 / 10 / 2009$ & & HCJ-161-09 \\
\hline GWA042012X & USGS-109 & 621 & Arsenic & 1.6 & & $U$ & & UG/L & $06 / 10 / 2009$ & & HCJ-161-09 \\
\hline GWA042012X & USGS-109 & 621 & Barium & 30.9 & & B & & UG/L & 06/10/2009 & & HCJ-161-09 \\
\hline GWA042012X & USGS-109 & 621 & Beryllium & 0.1 & & U & & UG/L & $06 / 10 / 2009$ & & HCJ-161-09 \\
\hline GWA042012X & USGS-109 & 621 & Cadmium & 0.11 & & U & & UG/L & $06 / 10 / 2009$ & & HCJ-161-09 \\
\hline GWA042012X & USGS-109 & 621 & Calcium & 36000 & & & & UG/L & $06 / 10 / 2009$ & & HCJ-161-09 \\
\hline GWA042012X & USGS-109 & 621 & Chromium & 5.85 & & & & UG/L & 06/10/2009 & & HCJ-161-09 \\
\hline GWA042012X & USGS-109 & 621 & Cobalt & 3.23 & & B & & UG/L & $06 / 10 / 2009$ & & HCJ-161-09 \\
\hline GWA042012X & USGS-109 & 621 & Copper & 0.571 & & B & & UG/L & $06 / 10 / 2009$ & & HCJ-161-09 \\
\hline GWA042012X & USGS-109 & 621 & Iron & 202 & & & & UG/L & 06/10/2009 & & HCJ-161-09 \\
\hline GWA042012X & USGS-109 & 621 & Lead & 0.5 & & U & & UG/L & $06 / 10 / 2009$ & & HCJ-161-09 \\
\hline GWA042012X & USGS-109 & 621 & Magnesium & 16300 & & & & UG/L & $06 / 10 / 2009$ & & HCJ-161-09 \\
\hline GWA042012X & USGS-109 & 621 & Manganese & 8.58 & & B & & UG/L & $06 / 10 / 2009$ & & HCJ-161-09 \\
\hline GWA042012X & USGS-109 & 621 & Mercury & 0.067 & & 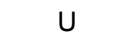 & & UG/L & $06 / 10 / 2009$ & & HCJ-161-09 \\
\hline GWA042012X & USGS-109 & 621 & Nickel & 1.04 & & B & & UG/L & $06 / 10 / 2009$ & & HCJ-161-09 \\
\hline GWA042012X & USGS-109 & 621 & Potassium & 2520 & & B & & UG/L & $06 / 10 / 2009$ & & HCJ-161-09 \\
\hline GWA042012X & USGS-109 & 621 & Selenium & 1 & & B & & UG/L & $06 / 10 / 2009$ & & HCJ-161-09 \\
\hline GWA042012X & USGS-109 & 621 & Silver & 0.2 & & U & & UG/L & $06 / 10 / 2009$ & & HCJ-161-09 \\
\hline GWA042012X & USGS-109 & 621 & Sodium & 11200 & & & & UG/L & $06 / 10 / 2009$ & & HCJ-161-09 \\
\hline GWA042012X & USGS-109 & 621 & Strontium & 219 & & & & UG/L & $06 / 10 / 2009$ & & HCJ-161-09 \\
\hline
\end{tabular}


Table A-2. (continued).

\begin{tabular}{|c|c|c|c|c|c|c|c|c|c|c|c|}
\hline $\begin{array}{c}\text { Field Sample } \\
\text { Number }\end{array}$ & Location & Depth & Compound & $\begin{array}{c}\text { Sample } \\
\text { Result }\end{array}$ & $\begin{array}{c}\text { Sample } \\
\text { Error }\end{array}$ & $\begin{array}{c}\text { Result } \\
\text { Qualifier }\end{array}$ & $\begin{array}{c}\text { Validation } \\
\text { Flag }\end{array}$ & $\begin{array}{c}\text { Sample } \\
\text { Units }\end{array}$ & $\begin{array}{c}\text { Date Sample } \\
\text { Collected }\end{array}$ & MDA & L\&V Report Number \\
\hline GWA042012X & USGS-109 & 621 & Thallium & 0.3 & & $U$ & & UG/L & $06 / 10 / 2009$ & & HCJ-161-09 \\
\hline GWA042012X & USGS-109 & 621 & Uranium & 2.43 & & B & & UG/L & 06/10/2009 & & HCJ-161-09 \\
\hline GWA042012X & USGS-109 & 621 & Vanadium & 3.15 & & B & & UG/L & 06/10/2009 & & HCJ-161-09 \\
\hline GWA042012X & USGS-109 & 621 & Zinc & 3.34 & & B & & UG/L & 06/10/2009 & & HCJ-161-09 \\
\hline GWA04201A1 & USGS-109 & 621 & Alkalinity, Total as $\mathrm{CaCO} 3$ & 139 & & & & $M G / L$ & 06/10/2009 & & HCJ-157-09 \\
\hline GWA04201AN & USGS-109 & 621 & Bromide & 0 & & $\mathrm{U}$ & & $M G / L$ & 06/10/2009 & & HCJ-159-09 \\
\hline GWA04201AN & USGS-109 & 621 & Chloride & 13.2 & & & & $M G / L$ & 06/10/2009 & & HCJ-159-09 \\
\hline GWA04201AN & USGS-109 & 621 & Fluoride & 0.25 & & $\mathrm{~J}$ & & $M G / L$ & 06/10/2009 & & HCJ-159-09 \\
\hline GWA04201AN & USGS-109 & 621 & Sulfate & 26.1 & & & & $M G / L$ & 06/10/2009 & & HCJ-159-09 \\
\hline GWA04201N2 & USGS-109 & 621 & Nitrogen, Nitrate/Nitrite & 0.669 & & & & $M G / L$ & 06/10/2009 & & HCJ-155-09 \\
\hline GWA04201VL & USGS-109 & 621 & Dichlorodifluoromethane & 1 & & $\mathrm{U}$ & & UG/L & 06/10/2009 & & HCJ-148-09 \\
\hline GWA04201VL & USGS-109 & 621 & Chloromethane & 1 & & U & & UG/L & 06/10/2009 & & HCJ-148-09 \\
\hline GWA04201VL & USGS-109 & 621 & Vinyl Chloride & 1 & & $u$ & & UG/L & 06/10/2009 & & HCJ-148-09 \\
\hline GWA04201VL & USGS-109 & 621 & Bromomethane & 1 & & $\mathrm{U}$ & & UG/L & 06/10/2009 & & HCJ-148-09 \\
\hline GWA04201VL & USGS-109 & 621 & Chloroethane & 1 & & U & & UG/L & 06/10/2009 & & HCJ-148-09 \\
\hline GWA04201VL & USGS-109 & 621 & Trichlorofluoromethane & 1 & & U & & UG/L & 06/10/2009 & & HCJ-148-09 \\
\hline GWA04201VL & USGS-109 & 621 & Acetone & 5 & & $U$ & & UG/L & 06/10/2009 & & HCJ-148-09 \\
\hline GWA04201VL & USGS-109 & 621 & 1,1-Dichloroethene & 1 & & $\mathrm{U}$ & & UG/L & 06/10/2009 & & HCJ-148-09 \\
\hline GWA04201VL & USGS-109 & 621 & Methyl acetate & 5 & & U & & UG/L & 06/10/2009 & & HCJ-148-09 \\
\hline GWA04201VL & USGS-109 & 621 & Methylene Chloride & 5 & & $u$ & & UG/L & 06/10/2009 & & HCJ-148-09 \\
\hline GWA04201VL & USGS-109 & 621 & Carbon disulfide & 5 & & $\mathrm{U}$ & & UG/L & 06/10/2009 & & HCJ-148-09 \\
\hline GWA04201VL & USGS-109 & 621 & Tert-butyl methyl ether & 1 & & U & & UG/L & 06/10/2009 & & HCJ-148-09 \\
\hline GWA04201VL & USGS-109 & 621 & trans-1,2-Dichloroethylene & 1 & & U & & UG/L & 06/10/2009 & & HCJ-148-09 \\
\hline GWA04201VL & USGS-109 & 621 & 1,1-Dichloroethane & 1 & & U & & UG/L & 06/10/2009 & & HCJ-148-09 \\
\hline GWA04201VL & USGS-109 & 621 & 2-Butanone & 5 & & $\mathrm{U}$ & & UG/L & 06/10/2009 & & HCJ-148-09 \\
\hline GWA04201VL & USGS-109 & 621 & cis-1,2-Dichloroethylene & 1 & & U & & UG/L & 06/10/2009 & & HCJ-148-09 \\
\hline GWA04201VL & USGS-109 & 621 & Chloroform & 1 & & $\mathrm{U}$ & & UG/L & 06/10/2009 & & HCJ-148-09 \\
\hline GWA04201VL & USGS-109 & 621 & 1,1,1-Trichloroethane & 1 & & $\mathrm{U}$ & & UG/L & 06/10/2009 & & HCJ-148-09 \\
\hline GWA04201VL & USGS-109 & 621 & Cyclohexane & 1 & & U & & UG/L & 06/10/2009 & & HCJ-148-09 \\
\hline GWA04201VL & USGS-109 & 621 & Carbon tetrachloride & 0.269 & & $\mathrm{~J}$ & & UG/L & 06/10/2009 & & HCJ-148-09 \\
\hline GWA04201VL & USGS-109 & 621 & 1,2-Dichloroethane & 1 & & $u$ & & UG/L & 06/10/2009 & & HCJ-148-09 \\
\hline GWA04201VL & USGS-109 & 621 & Benzene & 1 & & $\mathrm{U}$ & & UG/L & 06/10/2009 & & HCJ-148-09 \\
\hline GWA04201VL & USGS-109 & 621 & Trichloroethylene & 1 & & U & & UG/L & 06/10/2009 & & HCJ-148-09 \\
\hline GWA04201VL & USGS-109 & 621 & 1,2-Dichloropropane & 1 & & $u$ & & UG/L & 06/10/2009 & & HCJ-148-09 \\
\hline GWA04201VL & USGS-109 & 621 & Methyl cyclohexane & 1 & & $\mathrm{u}$ & & UG/L & 06/10/2009 & & HCJ-148-09 \\
\hline GWA04201VL & USGS-109 & 621 & Bromodichloromethane & 1 & & U & & UG/L & 06/10/2009 & & HCJ-148-09 \\
\hline GWA04201VL & USGS-109 & 621 & 4-Methyl-2-pentanone & 5 & & U & & UG/L & 06/10/2009 & & HCJ-148-09 \\
\hline GWA04201VL & USGS-109 & 621 & cis-1,3-Dichloropropylene & 1 & & $u$ & & UG/L & 06/10/2009 & & HCJ-148-09 \\
\hline GWA04201VL & USGS-109 & 621 & Toluene & 1 & & $\mathrm{u}$ & & UG/L & 06/10/2009 & & HCJ-148-09 \\
\hline GWA04201VL & USGS-109 & 621 & trans-1,3-Dichloropropylene & 1 & & U & & UG/L & 06/10/2009 & & HCJ-148-09 \\
\hline GWA04201VL & USGS-109 & 621 & 1,1,2-Trichloroethane & 1 & & $\mathrm{U}$ & & UG/L & 06/10/2009 & & HCJ-148-09 \\
\hline GWA04201VL & USGS-109 & 621 & 2-Hexanone & 5 & & $\mathrm{U}$ & & UG/L & 06/10/2009 & & HCJ-148-09 \\
\hline GWA04201VL & USGS-109 & 621 & Tetrachloroethylene & 1 & & $u$ & & UG/L & 06/10/2009 & & HCJ-148-09 \\
\hline GWA04201VL & USGS-109 & 621 & Dibromochloromethane & 1 & & U & & UG/L & 06/10/2009 & & HCJ-148-09 \\
\hline
\end{tabular}


Table A-2. (continued).

\begin{tabular}{|c|c|c|c|c|c|c|c|c|c|c|c|}
\hline $\begin{array}{c}\text { Field Sample } \\
\text { Number }\end{array}$ & Location & Depth & Compound & $\begin{array}{c}\text { Sample } \\
\text { Result }\end{array}$ & $\begin{array}{l}\text { Sample } \\
\text { Error }\end{array}$ & $\begin{array}{c}\text { Result } \\
\text { Qualifier }\end{array}$ & $\begin{array}{c}\text { Validation } \\
\text { Flag }\end{array}$ & $\begin{array}{c}\text { Sample } \\
\text { Units }\end{array}$ & $\begin{array}{c}\text { Date Sample } \\
\text { Collected }\end{array}$ & MDA & L\&V Report Number \\
\hline GWA04201VL & USGS-109 & 621 & 1,2-Dibromoethane & 1 & & $U$ & & UG/L & $06 / 10 / 2009$ & & HCJ-148-09 \\
\hline GWA04201VL & USGS-109 & 621 & Chlorobenzene & 1 & & U & & UG/L & 06/10/2009 & & HCJ-148-09 \\
\hline GWA04201VL & USGS-109 & 621 & Ethylbenzene & 1 & & $\mathrm{u}$ & & UG/L & $06 / 10 / 2009$ & & HCJ-148-09 \\
\hline GWA04201VL & USGS-109 & 621 & Styrene & 1 & & $\mathrm{U}$ & & UG/L & $06 / 10 / 2009$ & & HCJ-148-09 \\
\hline GWA04201VL & USGS-109 & 621 & Bromoform & 1 & & U & & UG/L & $06 / 10 / 2009$ & & HCJ-148-09 \\
\hline GWA04201VL & USGS-109 & 621 & 1,1,2,2-Tetrachloroethane & 1 & & $\mathrm{u}$ & & UG/L & $06 / 10 / 2009$ & & HCJ-148-09 \\
\hline GWA04201VL & USGS-109 & 621 & Isopropylbenzene & 1 & & $\mathrm{U}$ & & UG/L & $06 / 10 / 2009$ & & HCJ-148-09 \\
\hline GWA04201VL & USGS-109 & 621 & 1,3-Dichlorobenzene & 1 & & U & & UG/L & $06 / 10 / 2009$ & & HCJ-148-09 \\
\hline GWA04201VL & USGS-109 & 621 & 1,4-Dichlorobenzene & 1 & & U & & UG/L & $06 / 10 / 2009$ & & HCJ-148-09 \\
\hline GWA04201VL & USGS-109 & 621 & 1,2-Dibromo-3-chloropropane & 1 & & $\mathrm{u}$ & & UG/L & $06 / 10 / 2009$ & & HCJ-148-09 \\
\hline GWA04201VL & USGS-109 & 621 & Trichlorotrifluoroethane & 5 & & $\mathrm{U}$ & & UG/L & $06 / 10 / 2009$ & & HCJ-148-09 \\
\hline GWA04201VL & USGS-109 & 621 & Xylene (Total) & 1 & & $U$ & & UG/L & $06 / 10 / 2009$ & & HCJ-148-09 \\
\hline GWA04201VL & USGS-109 & 621 & 1,2,4-Trichlorobenzene & 1 & & U & & UG/L & 06/10/2009 & & HCJ-148-09 \\
\hline GWA04201VL & USGS-109 & 621 & 1,2-Dichlorobenzene & 1 & & $\mathrm{u}$ & & UG/L & $06 / 10 / 2009$ & & HCJ-148-09 \\
\hline GWA04201R8 & USGS-109 & 621 & Tritium & $1.01 \mathrm{E}+02$ & $9.11 \mathrm{E}+01$ & & U & $\mathrm{PCl} / \mathrm{L}$ & $06 / 10 / 2009$ & $3.04 \mathrm{E}+02$ & BAM-119-09 \\
\hline GWA04201RH & USGS-109 & 621 & Gross Alpha & 4.54E-01 & 4.96E-01 & & U & $\mathrm{PCl} / \mathrm{L}$ & $06 / 10 / 2009$ & $1.86 \mathrm{E}+00$ & BAM-120-09 \\
\hline GWA04201RH & USGS-109 & 621 & Gross Beta & $3.79 \mathrm{E}+00$ & 8.59E-01 & & & $\mathrm{PCl} / \mathrm{L}$ & 06/10/2009 & $2.08 \mathrm{E}+00$ & BAM-120-09 \\
\hline GWA04201RH & USGS-109 & 621 & Strontium-90 & $3.50 \mathrm{E}-01$ & $1.23 \mathrm{E}-01$ & & UJ & $\mathrm{PCl} / \mathrm{L}$ & $06 / 10 / 2009$ & $3.76 \mathrm{E}-01$ & BAM-120-09 \\
\hline GWA04201RH & USGS-109 & 621 & Technetium-99 & $-1.57 \mathrm{E}-01$ & $1.69 \mathrm{E}+00$ & & $\mathrm{U}$ & $\mathrm{PCl} / \mathrm{L}$ & $06 / 10 / 2009$ & $5.88 \mathrm{E}+00$ & BAM-120-09 \\
\hline GWA04201UX & USGS-109 & 621 & lodine-129 & $-5.29 E-02$ & 4.37E-02 & & U & $\mathrm{PCl} / \mathrm{L}$ & 06/10/2009 & 1.37E-01 & BAM-118-09 \\
\hline GWA043012X & USGS-110 & 566 & Aluminum & 15 & & $\mathrm{U}$ & & UG/L & $06 / 02 / 2009$ & & HCJ-161-09 \\
\hline GWA043012X & USGS-110 & 566 & Antimony & 1 & & U & & UG/L & $06 / 02 / 2009$ & & HCJ-161-09 \\
\hline GWA043012X & USGS-110 & 566 & Arsenic & 1.6 & & U & & UG/L & $06 / 02 / 2009$ & & HCJ-161-09 \\
\hline GWA043012X & USGS-110 & 566 & Barium & 34.8 & & B & & UG/L & $06 / 02 / 2009$ & & HCJ-161-09 \\
\hline GWA043012X & USGS-110 & 566 & Beryllium & 0.1 & & U & & UG/L & $06 / 02 / 2009$ & & HCJ-161-09 \\
\hline GWA043012X & USGS-110 & 566 & Cadmium & 0.11 & & $\mathrm{U}$ & & UG/L & $06 / 02 / 2009$ & & HCJ-161-09 \\
\hline GWA043012X & USGS-110 & 566 & Calcium & 31300 & & & & UG/L & $06 / 02 / 2009$ & & HCJ-161-09 \\
\hline GWA043012X & USGS-110 & 566 & Chromium & 2 & & U & & UG/L & $06 / 02 / 2009$ & & HCJ-161-09 \\
\hline GWA043012X & USGS-110 & 566 & Cobalt & 3.43 & & B & & UG/L & $06 / 02 / 2009$ & & HCJ-161-09 \\
\hline GWA043012X & USGS-110 & 566 & Copper & 0.604 & & B & & UG/L & $06 / 02 / 2009$ & & HCJ-161-09 \\
\hline GWA043012X & USGS-110 & 566 & Iron & 361 & & & & UG/L & $06 / 02 / 2009$ & & HCJ-161-09 \\
\hline GWA043012X & USGS-110 & 566 & Lead & 0.5 & & $\mathrm{U}$ & & UG/L & $06 / 02 / 2009$ & & HCJ-161-09 \\
\hline GWA043012X & USGS-110 & 566 & Magnesium & 13600 & & & & UG/L & $06 / 02 / 2009$ & & HCJ-161-09 \\
\hline GWA043012X & USGS-110 & 566 & Manganese & 20.8 & & & & UG/L & $06 / 02 / 2009$ & & HCJ-161-09 \\
\hline GWA043012X & USGS-110 & 566 & Mercury & 0.067 & & U & & UG/L & $06 / 02 / 2009$ & & HCJ-161-09 \\
\hline GWA043012X & USGS-110 & 566 & Nickel & 1.33 & & B & & UG/L & $06 / 02 / 2009$ & & HCJ-161-09 \\
\hline GWA043012X & USGS-110 & 566 & Potassium & 2940 & & B & & UG/L & $06 / 02 / 2009$ & & HCJ-161-09 \\
\hline GWA043012X & USGS-110 & 566 & Selenium & 1 & & U & & UG/L & $06 / 02 / 2009$ & & HCJ-161-09 \\
\hline GWA043012X & USGS-110 & 566 & Silver & 0.2 & & U & & UG/L & $06 / 02 / 2009$ & & HCJ-161-09 \\
\hline GWA043012X & USGS-110 & 566 & Sodium & 16100 & & & & UG/L & $06 / 02 / 2009$ & & HCJ-161-09 \\
\hline GWA043012X & USGS-110 & 566 & Strontium & 150 & & & & UG/L & $06 / 02 / 2009$ & & HCJ-161-09 \\
\hline GWA043012X & USGS-110 & 566 & Thallium & 0.3 & & U & & UG/L & $06 / 02 / 2009$ & & HCJ-161-09 \\
\hline GWA043012X & USGS-110 & 566 & Uranium & 2.31 & & B & & UG/L & $06 / 02 / 2009$ & & HCJ-161-09 \\
\hline GWA043012X & USGS-110 & 566 & Vanadium & 3 & & $U$ & & UG/L & $06 / 02 / 2009$ & & HCJ-161-09 \\
\hline
\end{tabular}


Table A-2. (continued).

\begin{tabular}{|c|c|c|c|c|c|c|c|c|c|c|c|}
\hline $\begin{array}{c}\text { Field Sample } \\
\text { Number }\end{array}$ & Location & Depth & Compound & $\begin{array}{l}\text { Sample } \\
\text { Result }\end{array}$ & $\begin{array}{c}\text { Sample } \\
\text { Error }\end{array}$ & $\begin{array}{c}\text { Result } \\
\text { Qualifier }\end{array}$ & $\begin{array}{c}\text { Validation } \\
\text { Flag }\end{array}$ & $\begin{array}{c}\text { Sample } \\
\text { Units }\end{array}$ & $\begin{array}{c}\text { Date Sample } \\
\text { Collected }\end{array}$ & MDA & L\&V Report Number \\
\hline GWA043012X & USGS-110 & 566 & Zinc & 3 & & $U$ & & UG/L & $06 / 02 / 2009$ & & HCJ-161-09 \\
\hline GWA04301A1 & USGS-110 & 566 & Alkalinity, Total as $\mathrm{CaCO} 3$ & 136 & & & & MG/L & $06 / 02 / 2009$ & & HCJ-157-09 \\
\hline GWA04301AN & USGS-110 & 566 & Bromide & 0 & & $\mathrm{u}$ & & $M G / L$ & $06 / 02 / 2009$ & & HCJ-159-09 \\
\hline GWA04301AN & USGS-110 & 566 & Chloride & 19.2 & & & & $M G / L$ & $06 / 02 / 2009$ & & HCJ-159-09 \\
\hline GWA04301AN & USGS-110 & 566 & Fluoride & 0.575 & & & & $M G / L$ & $06 / 02 / 2009$ & & HCJ-159-09 \\
\hline GWA04301AN & USGS-110 & 566 & Sulfate & 17.9 & & & & $M G / L$ & $06 / 02 / 2009$ & & HCJ-159-09 \\
\hline GWA04301N2 & USGS-110 & 566 & Nitrogen, Nitrate/Nitrite & 1.48 & & & & $M G / L$ & $06 / 02 / 2009$ & & HCJ-155-09 \\
\hline GWA04301VL & USGS-110 & 566 & Dichlorodifluoromethane & 1 & & U & & UG/L & $06 / 02 / 2009$ & & HCJ-148-09 \\
\hline GWA04301VL & USGS-110 & 566 & Chloromethane & 1 & & U & & UG/L & $06 / 02 / 2009$ & & HCJ-148-09 \\
\hline GWA04301VL & USGS-110 & 566 & Vinyl Chloride & 1 & & $\mathrm{u}$ & & UG/L & 06/02/2009 & & HCJ-148-09 \\
\hline GWA04301VL & USGS-110 & 566 & Bromomethane & 1 & & $\mathrm{U}$ & & UG/L & $06 / 02 / 2009$ & & HCJ-148-09 \\
\hline GWA04301VL & USGS-110 & 566 & Chloroethane & 1 & & U & & UG/L & $06 / 02 / 2009$ & & HCJ-148-09 \\
\hline GWA04301VL & USGS-110 & 566 & Trichlorofluoromethane & 1 & & $u$ & & UG/L & $06 / 02 / 2009$ & & HCJ-148-09 \\
\hline GWA04301VL & USGS-110 & 566 & Acetone & 5 & & $\mathrm{u}$ & & UG/L & $06 / 02 / 2009$ & & HCJ-148-09 \\
\hline GWA04301VL & USGS-110 & 566 & 1,1-Dichloroethene & 1 & & U & & UG/L & $06 / 02 / 2009$ & & HCJ-148-09 \\
\hline GWA04301VL & USGS-110 & 566 & Methyl acetate & 5 & & U & & UG/L & $06 / 02 / 2009$ & & HCJ-148-09 \\
\hline GWA04301VL & USGS-110 & 566 & Methylene Chloride & 5 & & $\mathrm{u}$ & & UG/L & $06 / 02 / 2009$ & & HCJ-148-09 \\
\hline GWA04301VL & USGS-110 & 566 & Carbon disulfide & 5 & & $\mathrm{U}$ & & UG/L & $06 / 02 / 2009$ & & HCJ-148-09 \\
\hline GWA04301VL & USGS-110 & 566 & Tert-butyl methyl ether & 1 & & U & & UG/L & $06 / 02 / 2009$ & & HCJ-148-09 \\
\hline GWA04301VL & USGS-110 & 566 & trans-1,2-Dichloroethylene & 1 & & $u$ & & UG/L & $06 / 02 / 2009$ & & HCJ-148-09 \\
\hline GWA04301VL & USGS-110 & 566 & 1,1-Dichloroethane & 1 & & $\mathrm{u}$ & & UG/L & $06 / 02 / 2009$ & & HCJ-148-09 \\
\hline GWA04301VL & USGS-110 & 566 & 2-Butanone & 5 & & U & & UG/L & $06 / 02 / 2009$ & & HCJ-148-09 \\
\hline GWA04301VL & USGS-110 & 566 & cis-1,2-Dichloroethylene & 1 & & U & & UG/L & $06 / 02 / 2009$ & & HCJ-148-09 \\
\hline GWA04301VL & USGS-110 & 566 & Chloroform & 1 & & U & & UG/L & $06 / 02 / 2009$ & & HCJ-148-09 \\
\hline GWA04301VL & USGS-110 & 566 & 1,1,1-Trichloroethane & 1 & & $\mathrm{U}$ & & UG/L & $06 / 02 / 2009$ & & HCJ-148-09 \\
\hline GWA04301VL & USGS-110 & 566 & Cyclohexane & 1 & & U & & UG/L & $06 / 02 / 2009$ & & HCJ-148-09 \\
\hline GWA04301VL & USGS-110 & 566 & Carbon tetrachloride & 1 & & $\mathrm{U}$ & & UG/L & $06 / 02 / 2009$ & & HCJ-148-09 \\
\hline GWA04301VL & USGS-110 & 566 & 1,2-Dichloroethane & 1 & & $\mathrm{u}$ & & UG/L & $06 / 02 / 2009$ & & HCJ-148-09 \\
\hline GWA04301VL & USGS-110 & 566 & Benzene & 1 & & U & & UG/L & $06 / 02 / 2009$ & & HCJ-148-09 \\
\hline GWA04301VL & USGS-110 & 566 & Trichloroethylene & 1 & & U & & UG/L & $06 / 02 / 2009$ & & HCJ-148-09 \\
\hline GWA04301VL & USGS-110 & 566 & 1,2-Dichloropropane & 1 & & $U$ & & UG/L & $06 / 02 / 2009$ & & HCJ-148-09 \\
\hline GWA04301VL & USGS-110 & 566 & Methyl cyclohexane & 1 & & $\mathrm{U}$ & & UG/L & $06 / 02 / 2009$ & & HCJ-148-09 \\
\hline GWA04301VL & USGS-110 & 566 & Bromodichloromethane & 1 & & U & & UG/L & $06 / 02 / 2009$ & & HCJ-148-09 \\
\hline GWA04301VL & USGS-110 & 566 & 4-Methyl-2-pentanone & 5 & & $U$ & & UG/L & $06 / 02 / 2009$ & & HCJ-148-09 \\
\hline GWA04301VL & USGS-110 & 566 & cis-1,3-Dichloropropylene & 1 & & $\mathrm{u}$ & & UG/L & $06 / 02 / 2009$ & & HCJ-148-09 \\
\hline GWA04301VL & USGS-110 & 566 & Toluene & 1 & & U & & UG/L & $06 / 02 / 2009$ & & HCJ-148-09 \\
\hline GWA04301VL & USGS-110 & 566 & trans-1,3-Dichloropropylene & 1 & & $\mathrm{U}$ & & UG/L & $06 / 02 / 2009$ & & HCJ-148-09 \\
\hline GWA04301VL & USGS-110 & 566 & 1,1,2-Trichloroethane & 1 & & 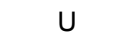 & & UG/L & $06 / 02 / 2009$ & & HCJ-148-09 \\
\hline GWA04301VL & USGS-110 & 566 & 2-Hexanone & 5 & & $\mathrm{U}$ & & UG/L & $06 / 02 / 2009$ & & HCJ-148-09 \\
\hline GWA04301VL & USGS-110 & 566 & Tetrachloroethylene & 1 & & $\mathrm{U}$ & & UG/L & $06 / 02 / 2009$ & & HCJ-148-09 \\
\hline GWA04301VL & USGS-110 & 566 & Dibromochloromethane & 1 & & $\mathrm{U}$ & & UG/L & $06 / 02 / 2009$ & & HCJ-148-09 \\
\hline GWA04301VL & USGS-110 & 566 & 1,2-Dibromoethane & 1 & & $\mathrm{U}$ & & UG/L & $06 / 02 / 2009$ & & HCJ-148-09 \\
\hline GWA04301VL & USGS-110 & 566 & Chlorobenzene & 1 & & 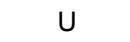 & & UG/L & $06 / 02 / 2009$ & & HCJ-148-09 \\
\hline GWA04301VL & USGS-110 & 566 & Ethylbenzene & 1 & & U & & UG/L & $06 / 02 / 2009$ & & HCJ-148-09 \\
\hline
\end{tabular}


Table A-2. (continued).

\begin{tabular}{|c|c|c|c|c|c|c|c|c|c|c|c|}
\hline $\begin{array}{c}\text { Field Sample } \\
\text { Number }\end{array}$ & Location & Depth & Compound & $\begin{array}{l}\text { Sample } \\
\text { Result }\end{array}$ & $\begin{array}{l}\text { Sample } \\
\text { Error }\end{array}$ & $\begin{array}{c}\text { Result } \\
\text { Qualifier }\end{array}$ & $\begin{array}{c}\text { Validation } \\
\text { Flag }\end{array}$ & $\begin{array}{c}\text { Sample } \\
\text { Units }\end{array}$ & $\begin{array}{c}\text { Date Sample } \\
\text { Collected }\end{array}$ & MDA & L\&V Report Number \\
\hline GWA04301VL & USGS-110 & 566 & Styrene & 1 & & $\mathrm{U}$ & & UG/L & $06 / 02 / 2009$ & & HCJ-148-09 \\
\hline GWA04301VL & USGS-110 & 566 & Bromoform & 1 & & U & & UG/L & $06 / 02 / 2009$ & & HCJ-148-09 \\
\hline GWA04301VL & USGS-110 & 566 & 1,1,2,2-Tetrachloroethane & 1 & & $u$ & & UG/L & $06 / 02 / 2009$ & & HCJ-148-09 \\
\hline GWA04301VL & USGS-110 & 566 & Isopropylbenzene & 1 & & $\mathrm{U}$ & & UG/L & $06 / 02 / 2009$ & & HCJ-148-09 \\
\hline GWA04301VL & USGS-110 & 566 & 1,3-Dichlorobenzene & 1 & & U & & UG/L & $06 / 02 / 2009$ & & HCJ-148-09 \\
\hline GWA04301VL & USGS-110 & 566 & 1,4-Dichlorobenzene & 1 & & $u$ & & UG/L & $06 / 02 / 2009$ & & HCJ-148-09 \\
\hline GWA04301VL & USGS-110 & 566 & 1,2-Dibromo-3-chloropropane & 1 & & 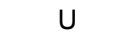 & & UG/L & 06/02/2009 & & HCJ-148-09 \\
\hline GWA04301VL & USGS-110 & 566 & Trichlorotrifluoroethane & 5 & & U & & UG/L & $06 / 02 / 2009$ & & HCJ-148-09 \\
\hline GWA04301VL & USGS-110 & 566 & Xylene (Total) & 1 & & U & & UG/L & $06 / 02 / 2009$ & & HCJ-148-09 \\
\hline GWA04301VL & USGS-110 & 566 & 1,2,4-Trichlorobenzene & 1 & & $u$ & & UG/L & $06 / 02 / 2009$ & & HCJ-148-09 \\
\hline GWA04301VL & USGS-110 & 566 & 1,2-Dichlorobenzene & 1 & & $U$ & & UG/L & $06 / 02 / 2009$ & & HCJ-148-09 \\
\hline GWA04301R8 & USGS-110 & 566 & Tritium & $0.00 \mathrm{E}+00$ & $8.83 E+01$ & & $\mathrm{U}$ & $\mathrm{PCI} / \mathrm{L}$ & $06 / 02 / 2009$ & $3.07 \mathrm{E}+02$ & BAM-119-09 \\
\hline GWA04301RH & USGS-110 & 566 & Gross Alpha & $2.02 \mathrm{E}+00$ & 7.41E-01 & & $\mathrm{J}$ & $\mathrm{PCl} / \mathrm{L}$ & $06 / 02 / 2009$ & $1.62 \mathrm{E}+00$ & BAM-120-09 \\
\hline GWA04301RH & USGS-110 & 566 & Gross Beta & $4.23 \mathrm{E}+00$ & $1.03 E+00$ & & & $\mathrm{PCl} / \mathrm{L}$ & $06 / 02 / 2009$ & $2.76 \mathrm{E}+00$ & BAM-120-09 \\
\hline GWA04301RH & USGS-110 & 566 & Strontium-90 & 1.36E-01 & $1.02 \mathrm{E}-01$ & & U & $\mathrm{PCl} / \mathrm{L}$ & $06 / 02 / 2009$ & $3.41 \mathrm{E}-01$ & BAM-120-09 \\
\hline GWA04301RH & USGS-110 & 566 & Technetium-99 & $-2.47 \mathrm{E}+00$ & $1.59 \mathrm{E}+00$ & & $U$ & $\mathrm{PCl} / \mathrm{L}$ & $06 / 02 / 2009$ & $5.81 \mathrm{E}+00$ & BAM-120-09 \\
\hline GWA04301UX & USGS-110 & 566 & lodine-129 & $-2.57 \mathrm{E}-02$ & 2.30E-02 & & $u$ & $\mathrm{PCl} / \mathrm{L}$ & $06 / 02 / 2009$ & 6.91E-02 & BAM-118-09 \\
\hline GWA05001A1 & USGS-132 & 646.7 & Alkalinity, Total as $\mathrm{CaCO} 3$ & 148 & & & & $M G / L$ & $06 / 24 / 2009$ & & HCJ-156-09 \\
\hline GWA05001AN & USGS-132 & 646.7 & Bromide & 0 & & U & & $M G / L$ & $06 / 24 / 2009$ & & HCJ-158-09 \\
\hline GWA05001AN & USGS-132 & 646.7 & Chloride & 17.5 & & & & $M G / L$ & $06 / 24 / 2009$ & & HCJ-158-09 \\
\hline GWA05001AN & USGS-132 & 646.7 & Fluoride & 0.28 & & $\mathrm{~J}$ & & $M G / L$ & $06 / 24 / 2009$ & & HCJ-158-09 \\
\hline GWA05001AN & USGS-132 & 646.7 & Sulfate & 37.2 & & & & $M G / L$ & $06 / 24 / 2009$ & & HCJ-158-09 \\
\hline GWA05001N2 & USGS-132 & 646.7 & Nitrogen, Nitrate/Nitrite & 0.905 & & & & $M G / L$ & $06 / 24 / 2009$ & & HCJ-154-09 \\
\hline GWA05101A1 & USGS-132 & 774.2 & Alkalinity, Total as $\mathrm{CaCO} 3$ & 139 & & & & MG/L & 06/29/2009 & & HCJ-168-09 \\
\hline GWA05201A1 & USGS-132 & 836 & Alkalinity, Total as $\mathrm{CaCO} 3$ & 141 & & & & $M G / L$ & 06/29/2009 & & HCJ-168-09 \\
\hline GWA05101AN & USGS-132 & 774.2 & Bromide & 0 & & U & & $M G / L$ & $06 / 29 / 2009$ & & HCJ-170-09 \\
\hline GWA05101AN & USGS-132 & 774.2 & Chloride & 10.5 & & & $\mathrm{~J}$ & MG/L & $06 / 29 / 2009$ & & HCJ-170-09 \\
\hline GWA05101AN & USGS-132 & 774.2 & Fluoride & 0.228 & & $\mathrm{~J}$ & & $M G / L$ & $06 / 29 / 2009$ & & HCJ-170-09 \\
\hline GWA05101AN & USGS-132 & 774.2 & Sulfate & 25.2 & & & & $M G / L$ & $06 / 29 / 2009$ & & HCJ-170-09 \\
\hline GWA05201AN & USGS-132 & 836 & Bromide & 0 & & U & & $M G / L$ & $06 / 29 / 2009$ & & HCJ-170-09 \\
\hline GWA05201AN & USGS-132 & 836 & Chloride & 9.52 & & & $\mathrm{~J}$ & MG/L & $06 / 29 / 2009$ & & HCJ-170-09 \\
\hline GWA05201AN & USGS-132 & 836 & Fluoride & 0.216 & & $\mathrm{~J}$ & & $M G / L$ & $06 / 29 / 2009$ & & HCJ-170-09 \\
\hline GWA05201AN & USGS-132 & 836 & Sulfate & 23.9 & & & & $M G / L$ & $06 / 29 / 2009$ & & HCJ-170-09 \\
\hline GWA05101N2 & USGS-132 & 774.2 & Nitrogen, Nitrate/Nitrite & 0.73 & & & & $M G / L$ & $06 / 29 / 2009$ & & HCJ-171-09 \\
\hline GWA05201N2 & USGS-132 & 836 & Nitrogen, Nitrate/Nitrite & 0.673 & & & & $M G / L$ & $06 / 29 / 2009$ & & HCJ-171-09 \\
\hline GWA050012X & USGS-132 & 646.7 & Aluminum & 15 & & U & & UG/L & $06 / 24 / 2009$ & & HCJ-160-09 \\
\hline GWA050012X & USGS-132 & 646.7 & Antimony & 1 & & U & & UG/L & $06 / 24 / 2009$ & & HCJ-160-09 \\
\hline GWA050012X & USGS-132 & 646.7 & Arsenic & 3 & & B & $U$ & UG/L & $06 / 24 / 2009$ & & HCJ-160-09 \\
\hline GWA050012X & USGS-132 & 646.7 & Barium & 55.5 & & B & & UG/L & $06 / 24 / 2009$ & & HCJ-160-09 \\
\hline GWA050012X & USGS-132 & 646.7 & Beryllium & 0.1 & & U & & UG/L & $06 / 24 / 2009$ & & HCJ-160-09 \\
\hline GWA050012X & USGS-132 & 646.7 & Cadmium & 0.11 & & $u$ & & UG/L & $06 / 24 / 2009$ & & HCJ-160-09 \\
\hline GWA050012X & USGS-132 & 646.7 & Calcium & 33200 & & & & UG/L & $06 / 24 / 2009$ & & HCJ-160-09 \\
\hline GWA050012X & USGS-132 & 646.7 & Chromium & 9.54 & & & & UG/L & $06 / 24 / 2009$ & & HCJ-160-09 \\
\hline GWA050012X & USGS-132 & 646.7 & Cobalt & 0.1 & & $U$ & & UG/L & $06 / 24 / 2009$ & & HCJ-160-09 \\
\hline
\end{tabular}


Table A-2. (continued).

\begin{tabular}{|c|c|c|c|c|c|c|c|c|c|c|c|}
\hline $\begin{array}{c}\text { Field Sample } \\
\text { Number }\end{array}$ & Location & Depth & Compound & $\begin{array}{l}\text { Sample } \\
\text { Result }\end{array}$ & $\begin{array}{c}\text { Sample } \\
\text { Error }\end{array}$ & $\begin{array}{c}\text { Result } \\
\text { Qualifier }\end{array}$ & $\begin{array}{c}\text { Validation } \\
\text { Flag }\end{array}$ & $\begin{array}{c}\text { Sample } \\
\text { Units }\end{array}$ & $\begin{array}{c}\text { Date Sample } \\
\text { Collected }\end{array}$ & MDA & L\&V Report Number \\
\hline GWA050012X & USGS-132 & 646.7 & Copper & 0.815 & & $\mathrm{~B}$ & $U$ & UG/L & 06/24/2009 & & HCJ-160-09 \\
\hline GWA050012X & USGS-132 & 646.7 & Iron & 87 & & B & & UG/L & 06/24/2009 & & HCJ-160-09 \\
\hline GWA050012X & USGS-132 & 646.7 & Lead & 0.5 & & $\mathrm{U}$ & & UG/L & 06/24/2009 & & HCJ-160-09 \\
\hline GWA050012X & USGS-132 & 646.7 & Magnesium & 15700 & & & & UG/L & 06/24/2009 & & HCJ-160-09 \\
\hline GWA050012X & USGS-132 & 646.7 & Manganese & 1 & & $U$ & & UG/L & 06/24/2009 & & HCJ-160-09 \\
\hline GWA050012X & USGS-132 & 646.7 & Mercury & 0.066 & & $\mathrm{U}$ & & UG/L & 06/24/2009 & & HCJ-160-09 \\
\hline GWA050012X & USGS-132 & 646.7 & Nickel & 0.756 & & B & & UG/L & 06/24/2009 & & HCJ-160-09 \\
\hline GWA050012X & USGS-132 & 646.7 & Potassium & 3510 & & B & & UG/L & 06/24/2009 & & HCJ-160-09 \\
\hline GWA050012X & USGS-132 & 646.7 & Selenium & 1 & & U & & UG/L & 06/24/2009 & & HCJ-160-09 \\
\hline GWA050012X & USGS-132 & 646.7 & Silver & 0.2 & & U & & UG/L & 06/24/2009 & & HCJ-160-09 \\
\hline GWA050012X & USGS-132 & 646.7 & Sodium & 24700 & & & & UG/L & 06/24/2009 & & HCJ-160-09 \\
\hline GWA050012X & USGS-132 & 646.7 & Strontium & 223 & & & & UG/L & 06/24/2009 & & HCJ-160-09 \\
\hline GWA050012X & USGS-132 & 646.7 & Thallium & 0.3 & & U & & UG/L & 06/24/2009 & & HCJ-160-09 \\
\hline GWA050012X & USGS-132 & 646.7 & Uranium & 3.06 & & & & UG/L & 06/24/2009 & & HCJ-160-09 \\
\hline GWA050012X & USGS-132 & 646.7 & Vanadium & 3.07 & & B & & UG/L & 06/24/2009 & & HCJ-160-09 \\
\hline GWA050012X & USGS-132 & 646.7 & Zinc & 6.12 & & B & & UG/L & 06/24/2009 & & HCJ-160-09 \\
\hline GWA051012X & USGS-132 & 774.2 & Aluminum & 15 & & $U$ & & UG/L & 06/29/2009 & & HCJ-172-09 \\
\hline GWA051012X & USGS-132 & 774.2 & Antimony & 1 & & U & & UG/L & 06/29/2009 & & HCJ-172-09 \\
\hline GWA051012X & USGS-132 & 774.2 & Arsenic & 1.6 & & $U$ & & UG/L & 06/29/2009 & & HCJ-172-09 \\
\hline GWA051012X & USGS-132 & 774.2 & Barium & 43.9 & & B & & UG/L & 06/29/2009 & & HCJ-172-09 \\
\hline GWA051012X & USGS-132 & 774.2 & Beryllium & 0.1 & & U & & UG/L & 06/29/2009 & & HCJ-172-09 \\
\hline GWA051012X & USGS-132 & 774.2 & Cadmium & 0.11 & & U & & UG/L & 06/29/2009 & & HCJ-172-09 \\
\hline GWA051012X & USGS-132 & 774.2 & Calcium & 40100 & & & & UG/L & 06/29/2009 & & HCJ-172-09 \\
\hline GWA051012X & USGS-132 & 774.2 & Chromium & 8.18 & & & & UG/L & 06/29/2009 & & HCJ-172-09 \\
\hline GWA051012X & USGS-132 & 774.2 & Cobalt & 0.16 & & B & & UG/L & 06/29/2009 & & HCJ-172-09 \\
\hline GWA051012X & USGS-132 & 774.2 & Copper & 0.853 & & B & & UG/L & 06/29/2009 & & HCJ-172-09 \\
\hline GWA051012X & USGS-132 & 774.2 & Iron & 232 & & & & UG/L & 06/29/2009 & & HCJ-172-09 \\
\hline GWA051012X & USGS-132 & 774.2 & Lead & 0.5 & & U & & UG/L & 06/29/2009 & & HCJ-172-09 \\
\hline GWA051012X & USGS-132 & 774.2 & Magnesium & 17500 & & & & UG/L & 06/29/2009 & & HCJ-172-09 \\
\hline GWA051012X & USGS-132 & 774.2 & Manganese & 1 & & $U$ & & UG/L & 06/29/2009 & & HCJ-172-09 \\
\hline GWA051012X & USGS-132 & 774.2 & Mercury & 0.066 & & $U$ & & UG/L & 06/29/2009 & & HCJ-172-09 \\
\hline GWA051012X & USGS-132 & 774.2 & Nickel & 1.39 & & B & & UG/L & 06/29/2009 & & HCJ-172-09 \\
\hline GWA051012X & USGS-132 & 774.2 & Potassium & 2670 & & B & & UG/L & 06/29/2009 & & HCJ-172-09 \\
\hline GWA051012X & USGS-132 & 774.2 & Selenium & 1.39 & & B & & UG/L & 06/29/2009 & & HCJ-172-09 \\
\hline GWA051012X & USGS-132 & 774.2 & Silver & 0.2 & & U & & UG/L & 06/29/2009 & & HCJ-172-09 \\
\hline GWA051012X & USGS-132 & 774.2 & Sodium & 12300 & & $\mathrm{E}$ & $\mathrm{J}$ & UG/L & 06/29/2009 & & HCJ-172-09 \\
\hline GWA051012X & USGS-132 & 774.2 & Strontium & 214 & & & & UG/L & 06/29/2009 & & HCJ-172-09 \\
\hline GWA051012X & USGS-132 & 774.2 & Thallium & 0.373 & & B & $U$ & UG/L & 06/29/2009 & & HCJ-172-09 \\
\hline GWA051012X & USGS-132 & 774.2 & Uranium & 2.47 & & B & & UG/L & 06/29/2009 & & HCJ-172-09 \\
\hline GWA051012X & USGS-132 & 774.2 & Vanadium & 6.55 & & B & & UG/L & 06/29/2009 & & HCJ-172-09 \\
\hline GWA051012X & USGS-132 & 774.2 & Zinc & 6.1 & & B & & UG/L & 06/29/2009 & & HCJ-172-09 \\
\hline GWA052012X & USGS-132 & 836 & Aluminum & 15 & & U & & UG/L & 06/29/2009 & & HCJ-172-09 \\
\hline GWA052012X & USGS-132 & 836 & Antimony & 1 & & $U$ & & UG/L & 06/29/2009 & & HCJ-172-09 \\
\hline GWA052012X & USGS-132 & 836 & Arsenic & 1.6 & & $U$ & & UG/L & 06/29/2009 & & HCJ-172-09 \\
\hline
\end{tabular}


Table A-2. (continued).

\begin{tabular}{|c|c|c|c|c|c|c|c|c|c|c|c|}
\hline $\begin{array}{c}\text { Field Sample } \\
\text { Number }\end{array}$ & Location & Depth & Compound & $\begin{array}{c}\text { Sample } \\
\text { Result }\end{array}$ & $\begin{array}{c}\text { Sample } \\
\text { Error }\end{array}$ & $\begin{array}{c}\text { Result } \\
\text { Qualifier }\end{array}$ & $\begin{array}{c}\text { Validation } \\
\text { Flag }\end{array}$ & $\begin{array}{c}\text { Sample } \\
\text { Units }\end{array}$ & $\begin{array}{c}\text { Date Sample } \\
\text { Collected }\end{array}$ & MDA & L\&V Report Number \\
\hline GWA052012X & USGS-132 & 836 & Barium & 43 & & $\mathrm{~B}$ & & UG/L & $06 / 29 / 2009$ & & HCJ-172-09 \\
\hline GWA052012X & USGS-132 & 836 & Beryllium & 0.1 & & U & & UG/L & 06/29/2009 & & HCJ-172-09 \\
\hline GWA052012X & USGS-132 & 836 & Cadmium & 0.11 & & $\mathrm{U}$ & & UG/L & 06/29/2009 & & HCJ-172-09 \\
\hline GWA052012X & USGS-132 & 836 & Calcium & 42000 & & & & UG/L & 06/29/2009 & & HCJ-172-09 \\
\hline GWA052012X & USGS-132 & 836 & Chromium & 7.91 & & & & UG/L & 06/29/2009 & & HCJ-172-09 \\
\hline GWA052012X & USGS-132 & 836 & Cobalt & 0.142 & & B & & UG/L & 06/29/2009 & & HCJ-172-09 \\
\hline GWA052012X & USGS-132 & 836 & Copper & 1.24 & & B & & UG/L & 06/29/2009 & & HCJ-172-09 \\
\hline GWA052012X & USGS-132 & 836 & Iron & 237 & & & & UG/L & 06/29/2009 & & HCJ-172-09 \\
\hline GWA052012X & USGS-132 & 836 & Lead & 0.5 & & U & & UG/L & 06/29/2009 & & HCJ-172-09 \\
\hline GWA052012X & USGS-132 & 836 & Magnesium & 16200 & & & & UG/L & 06/29/2009 & & HCJ-172-09 \\
\hline GWA052012X & USGS-132 & 836 & Manganese & 1 & & U & & UG/L & 06/29/2009 & & HCJ-172-09 \\
\hline GWA052012X & USGS-132 & 836 & Mercury & 0.066 & & $U$ & & UG/L & 06/29/2009 & & HCJ-172-09 \\
\hline GWA052012X & USGS-132 & 836 & Nickel & 1.39 & & B & & UG/L & 06/29/2009 & & HCJ-172-09 \\
\hline GWA052012X & USGS-132 & 836 & Potassium & 2390 & & B & & UG/L & 06/29/2009 & & HCJ-172-09 \\
\hline GWA052012X & USGS-132 & 836 & Selenium & 1.26 & & B & & UG/L & 06/29/2009 & & HCJ-172-09 \\
\hline GWA052012X & USGS-132 & 836 & Silver & 0.2 & & U & & UG/L & 06/29/2009 & & HCJ-172-09 \\
\hline GWA052012X & USGS-132 & 836 & Sodium & 8990 & & $\mathrm{E}$ & $\mathrm{J}$ & UG/L & 06/29/2009 & & HCJ-172-09 \\
\hline GWA052012X & USGS-132 & 836 & Strontium & 224 & & & & UG/L & 06/29/2009 & & HCJ-172-09 \\
\hline GWA052012X & USGS-132 & 836 & Thallium & 0.3 & & U & & UG/L & 06/29/2009 & & HCJ-172-09 \\
\hline GWA052012X & USGS-132 & 836 & Uranium & 2.49 & & B & & UG/L & 06/29/2009 & & HCJ-172-09 \\
\hline GWA052012X & USGS-132 & 836 & Vanadium & 6.23 & & B & & UG/L & 06/29/2009 & & HCJ-172-09 \\
\hline GWA052012X & USGS-132 & 836 & Zinc & 15.3 & & B & & UG/L & 06/29/2009 & & HCJ-172-09 \\
\hline GWA05001VL & USGS-132 & 646.7 & Dichlorodifluoromethane & 1 & & U & & UG/L & 06/24/2009 & & HCJ-153-09 \\
\hline GWA05001VL & USGS-132 & 646.7 & Chloromethane & 1 & & $\mathrm{U}$ & & UG/L & $06 / 24 / 2009$ & & HCJ-153-09 \\
\hline GWA05001VL & USGS-132 & 646.7 & Vinyl Chloride & 1 & & $\mathrm{U}$ & & UG/L & 06/24/2009 & & HCJ-153-09 \\
\hline GWA05001VL & USGS-132 & 646.7 & Bromomethane & 1 & & U & & UG/L & 06/24/2009 & & HCJ-153-09 \\
\hline GWA05001VL & USGS-132 & 646.7 & Chloroethane & 1 & & $\mathrm{U}$ & & UG/L & $06 / 24 / 2009$ & & HCJ-153-09 \\
\hline GWA05001VL & USGS-132 & 646.7 & Trichlorofluoromethane & 1 & & $\mathrm{U}$ & & UG/L & $06 / 24 / 2009$ & & HCJ-153-09 \\
\hline GWA05001VL & USGS-132 & 646.7 & Acetone & 5 & & U & & UG/L & 06/24/2009 & & HCJ-153-09 \\
\hline GWA05001VL & USGS-132 & 646.7 & 1,1-Dichloroethene & 1 & & U & & UG/L & 06/24/2009 & & HCJ-153-09 \\
\hline GWA05001VL & USGS-132 & 646.7 & Methyl acetate & 5 & & $u$ & & UG/L & 06/24/2009 & & HCJ-153-09 \\
\hline GWA05001VL & USGS-132 & 646.7 & Methylene Chloride & 5 & & $\mathrm{U}$ & & UG/L & 06/24/2009 & & HCJ-153-09 \\
\hline GWA05001VL & USGS-132 & 646.7 & Carbon disulfide & 5 & & U & & UG/L & 06/24/2009 & & HCJ-153-09 \\
\hline GWA05001VL & USGS-132 & 646.7 & Tert-butyl methyl ether & 1 & & $u$ & & UG/L & 06/24/2009 & & HCJ-153-09 \\
\hline GWA05001VL & USGS-132 & 646.7 & trans-1,2-Dichloroethylene & 1 & & $\mathrm{u}$ & & UG/L & $06 / 24 / 2009$ & & HCJ-153-09 \\
\hline GWA05001VL & USGS-132 & 646.7 & 1,1-Dichloroethane & 1 & & U & & UG/L & 06/24/2009 & & HCJ-153-09 \\
\hline GWA05001VL & USGS-132 & 646.7 & 2-Butanone & 5 & & U & & UG/L & 06/24/2009 & & HCJ-153-09 \\
\hline GWA05001VL & USGS-132 & 646.7 & cis-1,2-Dichloroethylene & 1 & & $u$ & & UG/L & 06/24/2009 & & HCJ-153-09 \\
\hline GWA05001VL & USGS-132 & 646.7 & Chloroform & 1 & & $\mathrm{u}$ & & UG/L & $06 / 24 / 2009$ & & HCJ-153-09 \\
\hline GWA05001VL & USGS-132 & 646.7 & 1,1,1-Trichloroethane & 1 & & U & & UG/L & 06/24/2009 & & HCJ-153-09 \\
\hline GWA05001VL & USGS-132 & 646.7 & Cyclohexane & 1 & & $\mathrm{U}$ & & UG/L & $06 / 24 / 2009$ & & HCJ-153-09 \\
\hline GWA05001VL & USGS-132 & 646.7 & Carbon tetrachloride & 0.452 & & $\mathrm{~J}$ & & UG/L & $06 / 24 / 2009$ & & HCJ-153-09 \\
\hline GWA05001VL & USGS-132 & 646.7 & 1,2-Dichloroethane & 1 & & 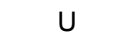 & & UG/L & 06/24/2009 & & HCJ-153-09 \\
\hline GWA05001VL & USGS-132 & 646.7 & Benzene & 1 & & U & & UG/L & 06/24/2009 & & HCJ-153-09 \\
\hline
\end{tabular}


Table A-2. (continued).

\begin{tabular}{|c|c|c|c|c|c|c|c|c|c|c|c|}
\hline $\begin{array}{c}\text { Field Sample } \\
\text { Number }\end{array}$ & Location & Depth & Compound & $\begin{array}{c}\text { Sample } \\
\text { Result }\end{array}$ & $\begin{array}{c}\text { Sample } \\
\text { Error }\end{array}$ & $\begin{array}{c}\text { Result } \\
\text { Qualifier }\end{array}$ & $\begin{array}{c}\text { Validation } \\
\text { Flag }\end{array}$ & $\begin{array}{c}\text { Sample } \\
\text { Units }\end{array}$ & $\begin{array}{c}\text { Date Sample } \\
\text { Collected }\end{array}$ & MDA & L\&V Report Number \\
\hline GWA05001VL & USGS-132 & 646.7 & Trichloroethylene & 1 & & $U$ & & UG/L & $06 / 24 / 2009$ & & HCJ-153-09 \\
\hline GWA05001VL & USGS-132 & 646.7 & 1,2-Dichloropropane & 1 & & U & & UG/L & $06 / 24 / 2009$ & & HCJ-153-09 \\
\hline GWA05001VL & USGS-132 & 646.7 & Methyl cyclohexane & 1 & & $\mathrm{u}$ & & UG/L & $06 / 24 / 2009$ & & HCJ-153-09 \\
\hline GWA05001VL & USGS-132 & 646.7 & Bromodichloromethane & 1 & & $\mathrm{U}$ & & UG/L & $06 / 24 / 2009$ & & HCJ-153-09 \\
\hline GWA05001VL & USGS-132 & 646.7 & 4-Methyl-2-pentanone & 5 & & U & & UG/L & $06 / 24 / 2009$ & & HCJ-153-09 \\
\hline GWA05001VL & USGS-132 & 646.7 & cis-1,3-Dichloropropylene & 1 & & $\mathrm{u}$ & & UG/L & $06 / 24 / 2009$ & & HCJ-153-09 \\
\hline GWA05001VL & USGS-132 & 646.7 & Toluene & 1 & & $\mathrm{U}$ & & UG/L & $06 / 24 / 2009$ & & HCJ-153-09 \\
\hline GWA05001VL & USGS-132 & 646.7 & trans-1,3-Dichloropropylene & 1 & & U & & UG/L & $06 / 24 / 2009$ & & HCJ-153-09 \\
\hline GWA05001VL & USGS-132 & 646.7 & 1,1,2-Trichloroethane & 1 & & U & & UG/L & $06 / 24 / 2009$ & & HCJ-153-09 \\
\hline GWA05001VL & USGS-132 & 646.7 & 2-Hexanone & 5 & & $\mathrm{u}$ & & UG/L & $06 / 24 / 2009$ & & HCJ-153-09 \\
\hline GWA05001VL & USGS-132 & 646.7 & Tetrachloroethylene & 1 & & $\mathrm{U}$ & & UG/L & $06 / 24 / 2009$ & & HCJ-153-09 \\
\hline GWA05001VL & USGS-132 & 646.7 & Dibromochloromethane & 1 & & U & & UG/L & $06 / 24 / 2009$ & & HCJ-153-09 \\
\hline GWA05001VL & USGS-132 & 646.7 & 1,2-Dibromoethane & 1 & & $u$ & & UG/L & $06 / 24 / 2009$ & & HCJ-153-09 \\
\hline GWA05001VL & USGS-132 & 646.7 & Chlorobenzene & 1 & & $\mathrm{u}$ & & UG/L & $06 / 24 / 2009$ & & HCJ-153-09 \\
\hline GWA05001VL & USGS-132 & 646.7 & Ethylbenzene & 1 & & U & & UG/L & $06 / 24 / 2009$ & & HCJ-153-09 \\
\hline GWA05001VL & USGS-132 & 646.7 & Styrene & 1 & & U & & UG/L & $06 / 24 / 2009$ & & HCJ-153-09 \\
\hline GWA05001VL & USGS-132 & 646.7 & Bromoform & 1 & & $U$ & & UG/L & $06 / 24 / 2009$ & & HCJ-153-09 \\
\hline GWA05001VL & USGS-132 & 646.7 & 1,1,2,2-Tetrachloroethane & 1 & & $\mathrm{u}$ & & UG/L & $06 / 24 / 2009$ & & HCJ-153-09 \\
\hline GWA05001VL & USGS-132 & 646.7 & Isopropylbenzene & 1 & & U & & UG/L & $06 / 24 / 2009$ & & HCJ-153-09 \\
\hline GWA05001VL & USGS-132 & 646.7 & 1,3-Dichlorobenzene & 1 & & $u$ & & UG/L & $06 / 24 / 2009$ & & HCJ-153-09 \\
\hline GWA05001VL & USGS-132 & 646.7 & 1,4-Dichlorobenzene & 1 & & $\mathrm{u}$ & & UG/L & $06 / 24 / 2009$ & & HCJ-153-09 \\
\hline GWA05001VL & USGS-132 & 646.7 & 1,2-Dibromo-3-chloropropane & 1 & & U & & UG/L & $06 / 24 / 2009$ & & HCJ-153-09 \\
\hline GWA05001VL & USGS-132 & 646.7 & Trichlorotrifluoroethane & 5 & & U & & UG/L & $06 / 24 / 2009$ & & HCJ-153-09 \\
\hline GWA05001VL & USGS-132 & 646.7 & Xylene (Total) & 1 & & U & & UG/L & $06 / 24 / 2009$ & & HCJ-153-09 \\
\hline GWA05001VL & USGS-132 & 646.7 & 1,2,4-Trichlorobenzene & 1 & & $\mathrm{U}$ & & UG/L & $06 / 24 / 2009$ & & HCJ-153-09 \\
\hline GWA05001VL & USGS-132 & 646.7 & 1,2-Dichlorobenzene & 1 & & U & & UG/L & $06 / 24 / 2009$ & & HCJ-153-09 \\
\hline GWA05101VL & USGS-132 & 774.2 & Dichlorodifluoromethane & 1 & & $\mathrm{U}$ & & UG/L & $06 / 29 / 2009$ & & HCJ-166-09 \\
\hline GWA05101VL & USGS-132 & 774.2 & Chloromethane & 1 & & $\mathrm{U}$ & & UG/L & $06 / 29 / 2009$ & & HCJ-166-09 \\
\hline GWA05101VL & USGS-132 & 774.2 & Vinyl Chloride & 1 & & U & & UG/L & $06 / 29 / 2009$ & & HCJ-166-09 \\
\hline GWA05101VL & USGS-132 & 774.2 & Bromomethane & 1 & & U & & UG/L & $06 / 29 / 2009$ & & HCJ-166-09 \\
\hline GWA05101VL & USGS-132 & 774.2 & Chloroethane & 1 & & $u$ & & UG/L & $06 / 29 / 2009$ & & HCJ-166-09 \\
\hline GWA05101VL & USGS-132 & 774.2 & Trichlorofluoromethane & 1 & & $\mathrm{u}$ & & UG/L & $06 / 29 / 2009$ & & HCJ-166-09 \\
\hline GWA05101VL & USGS-132 & 774.2 & Acetone & 5 & & U & & UG/L & $06 / 29 / 2009$ & & HCJ-166-09 \\
\hline GWA05101VL & USGS-132 & 774.2 & 1,1-Dichloroethene & 1 & & $U$ & & UG/L & $06 / 29 / 2009$ & & HCJ-166-09 \\
\hline GWA05101VL & USGS-132 & 774.2 & Methyl acetate & 5 & & $\mathrm{u}$ & & UG/L & $06 / 29 / 2009$ & & HCJ-166-09 \\
\hline GWA05101VL & USGS-132 & 774.2 & Methylene Chloride & 5 & & $\mathrm{U}$ & & UG/L & $06 / 29 / 2009$ & & HCJ-166-09 \\
\hline GWA05101VL & USGS-132 & 774.2 & Carbon disulfide & 5 & & U & & UG/L & $06 / 29 / 2009$ & & HCJ-166-09 \\
\hline GWA05101VL & USGS-132 & 774.2 & Tert-butyl methyl ether & 1 & & 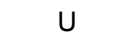 & & UG/L & $06 / 29 / 2009$ & & HCJ-166-09 \\
\hline GWA05101VL & USGS-132 & 774.2 & trans-1,2-Dichloroethylene & 1 & & $\mathrm{U}$ & & UG/L & $06 / 29 / 2009$ & & HCJ-166-09 \\
\hline GWA05101VL & USGS-132 & 774.2 & 1,1-Dichloroethane & 1 & & $\mathrm{U}$ & & UG/L & $06 / 29 / 2009$ & & HCJ-166-09 \\
\hline GWA05101VL & USGS-132 & 774.2 & 2-Butanone & 5 & & $\mathrm{U}$ & & UG/L & 06/29/2009 & & HCJ-166-09 \\
\hline GWA05101VL & USGS-132 & 774.2 & cis-1,2-Dichloroethylene & 1 & & $\mathrm{U}$ & & UG/L & $06 / 29 / 2009$ & & HCJ-166-09 \\
\hline GWA05101VL & USGS-132 & 774.2 & Chloroform & 1 & & 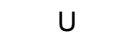 & & UG/L & $06 / 29 / 2009$ & & HCJ-166-09 \\
\hline GWA05101VL & USGS-132 & 774.2 & 1,1,1-Trichloroethane & 1 & & U & & UG/L & 06/29/2009 & & HCJ-166-09 \\
\hline
\end{tabular}


Table A-2. (continued).

\begin{tabular}{|c|c|c|c|c|c|c|c|c|c|c|c|}
\hline $\begin{array}{c}\text { Field Sample } \\
\text { Number }\end{array}$ & Location & Depth & Compound & $\begin{array}{c}\text { Sample } \\
\text { Result }\end{array}$ & $\begin{array}{c}\text { Sample } \\
\text { Error }\end{array}$ & $\begin{array}{c}\text { Result } \\
\text { Qualifier } \\
\end{array}$ & $\begin{array}{c}\text { Validation } \\
\text { Flag }\end{array}$ & $\begin{array}{c}\text { Sample } \\
\text { Units }\end{array}$ & $\begin{array}{c}\text { Date Sample } \\
\text { Collected }\end{array}$ & MDA & L\&V Report Number \\
\hline GWA05101VL & USGS-132 & 774.2 & Cyclohexane & 1 & & $U$ & & UG/L & $06 / 29 / 2009$ & & HCJ-166-09 \\
\hline GWA05101VL & USGS-132 & 774.2 & Carbon tetrachloride & 1 & & U & & UG/L & $06 / 29 / 2009$ & & HCJ-166-09 \\
\hline GWA05101VL & USGS-132 & 774.2 & 1,2-Dichloroethane & 1 & & $\mathrm{U}$ & & UG/L & $06 / 29 / 2009$ & & HCJ-166-09 \\
\hline GWA05101VL & USGS-132 & 774.2 & Benzene & 1 & & $\mathrm{U}$ & & UG/L & $06 / 29 / 2009$ & & HCJ-166-09 \\
\hline GWA05101VL & USGS-132 & 774.2 & Trichloroethylene & 1 & & U & & UG/L & $06 / 29 / 2009$ & & HCJ-166-09 \\
\hline GWA05101VL & USGS-132 & 774.2 & 1,2-Dichloropropane & 1 & & $\mathrm{U}$ & & UG/L & $06 / 29 / 2009$ & & HCJ-166-09 \\
\hline GWA05101VL & USGS-132 & 774.2 & Methyl cyclohexane & 1 & & $\mathrm{U}$ & & UG/L & $06 / 29 / 2009$ & & HCJ-166-09 \\
\hline GWA05101VL & USGS-132 & 774.2 & Bromodichloromethane & 1 & & U & & UG/L & $06 / 29 / 2009$ & & HCJ-166-09 \\
\hline GWA05101VL & USGS-132 & 774.2 & 4-Methyl-2-pentanone & 5 & & U & & UG/L & $06 / 29 / 2009$ & & HCJ-166-09 \\
\hline GWA05101VL & USGS-132 & 774.2 & cis-1,3-Dichloropropylene & 1 & & $\mathrm{U}$ & & UG/L & $06 / 29 / 2009$ & & HCJ-166-09 \\
\hline GWA05101VL & USGS-132 & 774.2 & Toluene & 1 & & $\mathrm{U}$ & & UG/L & $06 / 29 / 2009$ & & HCJ-166-09 \\
\hline GWA05101VL & USGS-132 & 774.2 & trans-1,3-Dichloropropylene & 1 & & U & & UG/L & $06 / 29 / 2009$ & & HCJ-166-09 \\
\hline GWA05101VL & USGS-132 & 774.2 & 1,1,2-Trichloroethane & 1 & & U & & UG/L & $06 / 29 / 2009$ & & HCJ-166-09 \\
\hline GWA05101VL & USGS-132 & 774.2 & 2-Hexanone & 5 & & $\mathrm{u}$ & & UG/L & $06 / 29 / 2009$ & & HCJ-166-09 \\
\hline GWA05101VL & USGS-132 & 774.2 & Tetrachloroethylene & 1 & & BJ & U & UG/L & $06 / 29 / 2009$ & & HCJ-166-09 \\
\hline GWA05101VL & USGS-132 & 774.2 & Dibromochloromethane & 1 & & $U$ & & UG/L & $06 / 29 / 2009$ & & HCJ-166-09 \\
\hline GWA05101VL & USGS-132 & 774.2 & 1,2-Dibromoethane & 1 & & $\mathrm{u}$ & & UG/L & $06 / 29 / 2009$ & & HCJ-166-09 \\
\hline GWA05101VL & USGS-132 & 774.2 & Chlorobenzene & 1 & & $\mathrm{U}$ & & UG/L & $06 / 29 / 2009$ & & HCJ-166-09 \\
\hline GWA05101VL & USGS-132 & 774.2 & Ethylbenzene & 1 & & U & & UG/L & $06 / 29 / 2009$ & & HCJ-166-09 \\
\hline GWA05101VL & USGS-132 & 774.2 & Styrene & 1 & & U & & UG/L & $06 / 29 / 2009$ & & HCJ-166-09 \\
\hline GWA05101VL & USGS-132 & 774.2 & Bromoform & 1 & & $\mathrm{u}$ & & UG/L & $06 / 29 / 2009$ & & HCJ-166-09 \\
\hline GWA05101VL & USGS-132 & 774.2 & 1,1,2,2-Tetrachloroethane & 1 & & U & & UG/L & $06 / 29 / 2009$ & & HCJ-166-09 \\
\hline GWA05101VL & USGS-132 & 774.2 & Isopropylbenzene & 1 & & U & & UG/L & $06 / 29 / 2009$ & & HCJ-166-09 \\
\hline GWA05101VL & USGS-132 & 774.2 & 1,3-Dichlorobenzene & 1 & & U & & UG/L & $06 / 29 / 2009$ & & HCJ-166-09 \\
\hline GWA05101VL & USGS-132 & 774.2 & 1,4-Dichlorobenzene & 1 & & $\mathrm{u}$ & & UG/L & $06 / 29 / 2009$ & & HCJ-166-09 \\
\hline GWA05101VL & USGS-132 & 774.2 & 1,2-Dibromo-3-chloropropane & 1 & & U & & UG/L & $06 / 29 / 2009$ & & HCJ-166-09 \\
\hline GWA05101VL & USGS-132 & 774.2 & Trichlorotrifluoroethane & 5 & & $\mathrm{U}$ & & UG/L & $06 / 29 / 2009$ & & HCJ-166-09 \\
\hline GWA05101VL & USGS-132 & 774.2 & Xylene (Total) & 1 & & $\mathrm{u}$ & & UG/L & $06 / 29 / 2009$ & & HCJ-166-09 \\
\hline GWA05101VL & USGS-132 & 774.2 & 1,2,4-Trichlorobenzene & 1 & & U & & UG/L & $06 / 29 / 2009$ & & HCJ-166-09 \\
\hline GWA05101VL & USGS-132 & 774.2 & 1,2-Dichlorobenzene & 1 & & U & & UG/L & $06 / 29 / 2009$ & & HCJ-166-09 \\
\hline GWA05101VL & USGS-132 & 774.2 & Unknown & 31.3 & & $\mathrm{~J}$ & & UG/L & $06 / 29 / 2009$ & & \\
\hline GWA05201VL & USGS-132 & 836 & Dichlorodifluoromethane & 1 & & $\mathrm{u}$ & & UG/L & $06 / 29 / 2009$ & & HCJ-166-09 \\
\hline GWA05201VL & USGS-132 & 836 & Chloromethane & 1 & & U & & UG/L & $06 / 29 / 2009$ & & HCJ-166-09 \\
\hline GWA05201VL & USGS-132 & 836 & Vinyl Chloride & 1 & & $U$ & & UG/L & $06 / 29 / 2009$ & & HCJ-166-09 \\
\hline GWA05201VL & USGS-132 & 836 & Bromomethane & 1 & & $\mathrm{u}$ & & UG/L & $06 / 29 / 2009$ & & HCJ-166-09 \\
\hline GWA05201VL & USGS-132 & 836 & Chloroethane & 1 & & $\mathrm{U}$ & & UG/L & $06 / 29 / 2009$ & & HCJ-166-09 \\
\hline GWA05201VL & USGS-132 & 836 & Trichlorofluoromethane & 1 & & U & & UG/L & $06 / 29 / 2009$ & & HCJ-166-09 \\
\hline GWA05201VL & USGS-132 & 836 & Acetone & 5 & & 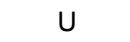 & & UG/L & $06 / 29 / 2009$ & & HCJ-166-09 \\
\hline GWA05201VL & USGS-132 & 836 & 1,1-Dichloroethene & 1 & & $\mathrm{U}$ & & UG/L & $06 / 29 / 2009$ & & HCJ-166-09 \\
\hline GWA05201VL & USGS-132 & 836 & Methyl acetate & 5 & & $\mathrm{U}$ & & UG/L & $06 / 29 / 2009$ & & HCJ-166-09 \\
\hline GWA05201VL & USGS-132 & 836 & Methylene Chloride & 5 & & $\mathrm{U}$ & & UG/L & 06/29/2009 & & HCJ-166-09 \\
\hline GWA05201VL & USGS-132 & 836 & Carbon disulfide & 5 & & $\mathrm{U}$ & & UG/L & $06 / 29 / 2009$ & & HCJ-166-09 \\
\hline GWA05201VL & USGS-132 & 836 & Tert-butyl methyl ether & 1 & & $\mathrm{U}$ & & UG/L & $06 / 29 / 2009$ & & HCJ-166-09 \\
\hline GWA05201VL & USGS-132 & 836 & trans-1,2-Dichloroethylene & 1 & & U & & UG/L & 06/29/2009 & & HCJ-166-09 \\
\hline
\end{tabular}


Table A-2. (continued).

\begin{tabular}{|c|c|c|c|c|c|c|c|c|c|c|c|}
\hline $\begin{array}{c}\text { Field Sample } \\
\text { Number }\end{array}$ & Location & Depth & Compound & $\begin{array}{l}\text { Sample } \\
\text { Result }\end{array}$ & $\begin{array}{l}\text { Sample } \\
\text { Error }\end{array}$ & $\begin{array}{c}\text { Result } \\
\text { Qualifier }\end{array}$ & $\begin{array}{c}\text { Validation } \\
\text { Flag }\end{array}$ & $\begin{array}{c}\text { Sample } \\
\text { Units }\end{array}$ & $\begin{array}{c}\text { Date Sample } \\
\text { Collected }\end{array}$ & MDA & L\&V Report Number \\
\hline GWA05201VL & USGS-132 & 836 & 1,1-Dichloroethane & 1 & & $\mathrm{U}$ & & UG/L & $06 / 29 / 2009$ & & HCJ-166-09 \\
\hline GWA05201VL & USGS-132 & 836 & 2-Butanone & 5 & & $u$ & & UG/L & $06 / 29 / 2009$ & & HCJ-166-09 \\
\hline GWA05201VL & USGS-132 & 836 & cis-1,2-Dichloroethylene & 1 & & $u$ & & UG/L & $06 / 29 / 2009$ & & HCJ-166-09 \\
\hline GWA05201VL & USGS-132 & 836 & Chloroform & 1 & & 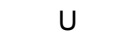 & & UG/L & $06 / 29 / 2009$ & & HCJ-166-09 \\
\hline GWA05201VL & USGS-132 & 836 & 1,1,1-Trichloroethane & 1 & & U & & UG/L & $06 / 29 / 2009$ & & HCJ-166-09 \\
\hline GWA05201VL & USGS-132 & 836 & Cyclohexane & 1 & & $u$ & & UG/L & $06 / 29 / 2009$ & & HCJ-166-09 \\
\hline GWA05201VL & USGS-132 & 836 & Carbon tetrachloride & 1 & & $u$ & & UG/L & $06 / 29 / 2009$ & & HCJ-166-09 \\
\hline GWA05201VL & USGS-132 & 836 & 1,2-Dichloroethane & 1 & & U & & UG/L & $06 / 29 / 2009$ & & HCJ-166-09 \\
\hline GWA05201VL & USGS-132 & 836 & Benzene & 1 & & U & & UG/L & $06 / 29 / 2009$ & & HCJ-166-09 \\
\hline GWA05201VL & USGS-132 & 836 & Trichloroethylene & 1 & & $u$ & & UG/L & $06 / 29 / 2009$ & & HCJ-166-09 \\
\hline GWA05201VL & USGS-132 & 836 & 1,2-Dichloropropane & 1 & & $U$ & & UG/L & $06 / 29 / 2009$ & & HCJ-166-09 \\
\hline GWA05201VL & USGS-132 & 836 & Methyl cyclohexane & 1 & & U & & UG/L & $06 / 29 / 2009$ & & HCJ-166-09 \\
\hline GWA05201VL & USGS-132 & 836 & Bromodichloromethane & 1 & & U & & UG/L & $06 / 29 / 2009$ & & HCJ-166-09 \\
\hline GWA05201VL & USGS-132 & 836 & 4-Methyl-2-pentanone & 5 & & $u$ & & UG/L & $06 / 29 / 2009$ & & HCJ-166-09 \\
\hline GWA05201VL & USGS-132 & 836 & cis-1,3-Dichloropropylene & 1 & & U & & UG/L & $06 / 29 / 2009$ & & HCJ-166-09 \\
\hline GWA05201VL & USGS-132 & 836 & Toluene & 1 & & U & & UG/L & $06 / 29 / 2009$ & & HCJ-166-09 \\
\hline GWA05201VL & USGS-132 & 836 & trans-1,3-Dichloropropylene & 1 & & $u$ & & UG/L & $06 / 29 / 2009$ & & HCJ-166-09 \\
\hline GWA05201VL & USGS-132 & 836 & 1,1,2-Trichloroethane & 1 & & $u$ & & UG/L & 06/29/2009 & & HCJ-166-09 \\
\hline GWA05201VL & USGS-132 & 836 & 2-Hexanone & 5 & & U & & UG/L & $06 / 29 / 2009$ & & HCJ-166-09 \\
\hline GWA05201VL & USGS-132 & 836 & Tetrachloroethylene & 1 & & BJ & $\mathrm{U}$ & UG/L & $06 / 29 / 2009$ & & HCJ-166-09 \\
\hline GWA05201VL & USGS-132 & 836 & Dibromochloromethane & 1 & & $U$ & & UG/L & $06 / 29 / 2009$ & & HCJ-166-09 \\
\hline GWA05201VL & USGS-132 & 836 & 1,2-Dibromoethane & 1 & & $\mathrm{U}$ & & UG/L & $06 / 29 / 2009$ & & HCJ-166-09 \\
\hline GWA05201VL & USGS-132 & 836 & Chlorobenzene & 1 & & U & & UG/L & $06 / 29 / 2009$ & & HCJ-166-09 \\
\hline GWA05201VL & USGS-132 & 836 & Ethylbenzene & 1 & & $u$ & & UG/L & $06 / 29 / 2009$ & & HCJ-166-09 \\
\hline GWA05201VL & USGS-132 & 836 & Styrene & 1 & & $U$ & & UG/L & 06/29/2009 & & HCJ-166-09 \\
\hline GWA05201VL & USGS-132 & 836 & Bromoform & 1 & & U & & UG/L & $06 / 29 / 2009$ & & HCJ-166-09 \\
\hline GWA05201VL & USGS-132 & 836 & 1,1,2,2-Tetrachloroethane & 1 & & $u$ & & UG/L & 06/29/2009 & & HCJ-166-09 \\
\hline GWA05201VL & USGS-132 & 836 & Isopropylbenzene & 1 & & $u$ & & UG/L & $06 / 29 / 2009$ & & HCJ-166-09 \\
\hline GWA05201VL & USGS-132 & 836 & 1,3-Dichlorobenzene & 1 & & $\mathrm{U}$ & & UG/L & $06 / 29 / 2009$ & & HCJ-166-09 \\
\hline GWA05201VL & USGS-132 & 836 & 1,4-Dichlorobenzene & 1 & & U & & UG/L & $06 / 29 / 2009$ & & HCJ-166-09 \\
\hline GWA05201VL & USGS-132 & 836 & 1,2-Dibromo-3-chloropropane & 1 & & U & & UG/L & $06 / 29 / 2009$ & & HCJ-166-09 \\
\hline GWA05201VL & USGS-132 & 836 & Trichlorotrifluoroethane & 5 & & 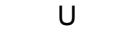 & & UG/L & 06/29/2009 & & HCJ-166-09 \\
\hline GWA05201VL & USGS-132 & 836 & Xylene (Total) & 1 & & $u$ & & UG/L & $06 / 29 / 2009$ & & HCJ-166-09 \\
\hline GWA05201VL & USGS-132 & 836 & 1,2,4-Trichlorobenzene & 1 & & U & & UG/L & $06 / 29 / 2009$ & & HCJ-166-09 \\
\hline GWA05201VL & USGS-132 & 836 & 1,2-Dichlorobenzene & 1 & & $u$ & & UG/L & $06 / 29 / 2009$ & & HCJ-166-09 \\
\hline GWA05201VL & USGS-132 & 836 & Unknown & 11.2 & & $\mathrm{~J}$ & & UG/L & $06 / 29 / 2009$ & & \\
\hline GWA05201VL & USGS-132 & 836 & Unknown & 18.3 & & $\mathrm{~J}$ & & UG/L & $06 / 29 / 2009$ & & \\
\hline GWA05001R8 & USGS-132 & 646.7 & Tritium & $1.77 \mathrm{E}+02$ & $1.18 \mathrm{E}+02$ & & U & $\mathrm{PCl} / \mathrm{L}$ & $06 / 24 / 2009$ & $3.87 \mathrm{E}+02$ & BAM-119-09 \\
\hline GWA05101R8 & USGS-132 & 774.2 & Tritium & $1.86 \mathrm{E}+02$ & $1.18 \mathrm{E}+02$ & & $u$ & $\mathrm{PCl} / \mathrm{L}$ & $06 / 29 / 2009$ & $3.85 \mathrm{E}+02$ & BAM-119-09 \\
\hline GWA05201R8 & USGS-132 & 836 & Tritium & $2.73 E+02$ & $1.23 E+02$ & & UJ & $\mathrm{PCl} / \mathrm{L}$ & $06 / 29 / 2009$ & $3.85 \mathrm{E}+02$ & BAM-119-09 \\
\hline GWA05001RH & USGS-132 & 646.7 & Gross Alpha & $2.90 \mathrm{E}+00$ & 8.91E-01 & & & $\mathrm{PCI} / \mathrm{L}$ & $06 / 24 / 2009$ & $1.83 \mathrm{E}+00$ & BAM-120-09 \\
\hline GWA05001RH & USGS-132 & 646.7 & Gross Beta & $4.55 E+00$ & 8.91E-01 & & & $\mathrm{PCl} / \mathrm{L}$ & $06 / 24 / 2009$ & $1.89 \mathrm{E}+00$ & BAM-120-09 \\
\hline GWA05101RH & USGS-132 & 774.2 & Gross Alpha & $1.46 \mathrm{E}+00$ & 7.08E-01 & & UJ & $\mathrm{PCI} / \mathrm{L}$ & $06 / 29 / 2009$ & $2.00 \mathrm{E}+00$ & BAM-120-09 \\
\hline GWA05101RH & USGS-132 & 774.2 & Gross Beta & $3.91 \mathrm{E}+00$ & $9.62 \mathrm{E}-01$ & & & $\mathrm{PCI} / \mathrm{L}$ & $06 / 29 / 2009$ & $2.56 \mathrm{E}+00$ & BAM-120-09 \\
\hline
\end{tabular}


Table A-2. (continued).

\begin{tabular}{|c|c|c|c|c|c|c|c|c|c|c|c|}
\hline $\begin{array}{c}\text { Field Sample } \\
\text { Number }\end{array}$ & Location & Depth & Compound & $\begin{array}{l}\text { Sample } \\
\text { Result }\end{array}$ & $\begin{array}{c}\text { Sample } \\
\text { Error }\end{array}$ & $\begin{array}{c}\text { Result } \\
\text { Qualifier }\end{array}$ & $\begin{array}{c}\text { Validation } \\
\text { Flag }\end{array}$ & $\begin{array}{c}\text { Sample } \\
\text { Units }\end{array}$ & $\begin{array}{c}\text { Date Sample } \\
\text { Collected }\end{array}$ & MDA & L\&V Report Number \\
\hline GWA05201RH & USGS-132 & 836 & Gross Alpha & $2.78 \mathrm{E}+00$ & $9.48 \mathrm{E}-01$ & & $\mathrm{~J}$ & $\mathrm{PCl} / \mathrm{L}$ & $06 / 29 / 2009$ & $2.35 \mathrm{E}+00$ & BAM-120-09 \\
\hline GWA05201RH & USGS-132 & 836 & Gross Beta & $3.65 \mathrm{E}+00$ & 8.78E-01 & & & $\mathrm{PCl} / \mathrm{L}$ & 06/29/2009 & $2.25 \mathrm{E}+00$ & BAM-120-09 \\
\hline GWA05001RH & USGS-132 & 646.7 & Strontium-90 & $1.74 \mathrm{E}-01$ & $1.05 \mathrm{E}-01$ & & $\mathrm{U}$ & $\mathrm{PCl} / \mathrm{L}$ & 06/24/2009 & 3.44E-01 & BAM-120-09 \\
\hline GWA05101RH & USGS-132 & 774.2 & Strontium-90 & $1.58 \mathrm{E}-01$ & $1.44 \mathrm{E}-01$ & & $\mathrm{U}$ & $\mathrm{PCl} / \mathrm{L}$ & 06/29/2009 & 4.82E-01 & BAM-120-09 \\
\hline GWA05201RH & USGS-132 & 836 & Strontium-90 & $-5.77 \mathrm{E}-02$ & 1.33E-01 & & $U$ & $\mathrm{PCl} / \mathrm{L}$ & 06/29/2009 & 4.64E-01 & BAM-120-09 \\
\hline GWA05001RH & USGS-132 & 646.7 & Technetium-99 & $-1.77 \mathrm{E}+00$ & $1.62 \mathrm{E}+00$ & & $\mathrm{U}$ & $\mathrm{PCl} / \mathrm{L}$ & 06/24/2009 & $5.82 \mathrm{E}+00$ & BAM-120-09 \\
\hline GWA05101RH & USGS-132 & 774.2 & Technetium-99 & $-4.66 \mathrm{E}+00$ & $2.78 \mathrm{E}+00$ & & $\mathrm{U}$ & $\mathrm{PCl} / \mathrm{L}$ & 06/29/2009 & $9.57 \mathrm{E}+00$ & BAM-120-09 \\
\hline GWA05201RH & USGS-132 & 836 & Technetium-99 & $-1.16 E+00$ & $2.86 \mathrm{E}+00$ & & $U$ & $\mathrm{PCl} / \mathrm{L}$ & 06/29/2009 & $9.69 E+00$ & BAM-120-09 \\
\hline GWA05001UX & USGS-132 & 646.7 & lodine-129 & $-1.16 \mathrm{E}-02$ & 2.47E-02 & & $U$ & $\mathrm{PCl} / \mathrm{L}$ & 06/24/2009 & 7.84E-02 & BAM-118-09 \\
\hline GWA05101UX & USGS-132 & 774.2 & lodine-129 & $-4.05 \mathrm{E}-03$ & $1.94 \mathrm{E}-02$ & & $\mathrm{U}$ & $\mathrm{PCl} / \mathrm{L}$ & 06/29/2009 & 6.37E-02 & BAM-118-09 \\
\hline GWA05201UX & USGS-132 & 836 & lodine-129 & $-2.37 \mathrm{E}-02$ & $1.94 \mathrm{E}-02$ & & $\mathrm{U}$ & $\mathrm{PCl} / \mathrm{L}$ & 06/29/2009 & $5.40 \mathrm{E}-02$ & BAM-118-09 \\
\hline
\end{tabular}




\section{Appendix B}

\section{Quality Assurance and Quality Control \\ Sample Results from Fiscal Year 2009 \\ Groundwater Sampling}


B-2 


\section{Appendix B}

\section{Quality Assurance and Quality Control Sample Results from Fiscal Year 2009 Groundwater Sampling}

\section{B-1. QUALITY ASSURANCE AND QUALITY CONTROL SAMPLING}

The purpose of collecting and analyzing quality assurance and quality control samples is to confirm that project objectives and data quality objectives have been achieved. Overall objectives associated with Idaho National Laboratory (INL) Waste Area Group (WAG) 10 annual groundwater monitoring are discussed in Groundwater Monitoring and Field Sampling Plan for Operable Unit 10-08 (DOE-ID 2007). The following subsections discuss overall objectives and quality assurance and quality control sample results for the Fiscal Year (FY) 2009 sampling effort.

\section{B-1.1 Precision and Accuracy}

Spatial variations in concentrations of contaminants at individual sites create sampling variability. Additional variability, called measurement error, occurs during sample collection, handling, processing, analysis, quality evaluation, and reporting. Concentrations of contaminants reported may differ from true concentrations in the media sampled as a result of measurement error, which can be minimized but not eliminated. Though it may not be significant in many cases, it is important to assess the contribution of measurement error to the total error in individual investigations. Analytical results of quality control samples are used to estimate (1) accuracy and precision, (2) quantitative descriptions of measurement error, and (3) bias.

\section{B-1.1.1 Overall Precision}

Precision is a measure of the reproducibility of measurements under a given set of conditions. In the field, precision is affected by sample-collection procedures and natural heterogeneity of the matrix. Overall precision (field and laboratory) can be evaluated by using duplicate samples collected in the field. Typically, greater precision is required for analytes with very low action levels that are close to background concentrations. Allowable laboratory precision for water samples is defined as having a relative percent difference (RPD) of less than or equal to $20 \%$. Field precision is the difference between overall precision and laboratory precision. Duplicate samples were taken at Wells USGS-104 and USGS-106. Table B-1 summarizes precision for the FY 2009 groundwater monitoring. Using the formula in Equation (B-1), RPD was calculated only for those samples that had true positive values for both the initial sample and the field duplicate:

$$
\mathrm{RPD}=\frac{|\mathrm{S}-\mathrm{D}|}{\mathrm{S}+\mathrm{D}} \times 200
$$

Where

$$
\begin{aligned}
& \mathrm{S}=\text { sample } \\
& \mathrm{D}=\text { duplicate. }
\end{aligned}
$$


Table B-1. Overall precision for Fiscal Year 2009 analytical data.

\begin{tabular}{|c|c|c|c|c|}
\hline Analyte & Units & Sample & Duplicate & $\begin{array}{c}\text { Relative Percent } \\
\text { Difference }\end{array}$ \\
\hline \multicolumn{5}{|l|}{ USGS-106 } \\
\hline Alkalinity, total as $\mathrm{CaCO}_{3}$ & $\mathrm{mg} / \mathrm{L}$ & 155 & 155 & 0.00 \\
\hline Barium & $\mu \mathrm{g} / \mathrm{L}$ & 46.8 & 47 & 0.43 \\
\hline Cadmium & $\mu \mathrm{g} / \mathrm{L}$ & 0.288 & 0.24 & 18.18 \\
\hline Calcium & $\mu \mathrm{g} / \mathrm{L}$ & 43,300 & 41,600 & 4.00 \\
\hline Chloride & $\mathrm{mg} / \mathrm{L}$ & 14.9 & 15 & 0.67 \\
\hline Chromium & $\mu \mathrm{g} / \mathrm{L}$ & 6.93 & 6.85 & 1.16 \\
\hline Cobalt & $\mu \mathrm{g} / \mathrm{L}$ & 4.33 & 0.229 & 179.91 \\
\hline Copper & $\mu \mathrm{g} / \mathrm{L}$ & 1.03 & 1.02 & 0.98 \\
\hline Fluoride & $\mathrm{mg} / \mathrm{L}$ & 0.211 & 0.192 & 9.43 \\
\hline Gross beta & $\mathrm{pCi} / \mathrm{L}$ & 3.42 & 2.59 & 27.62 \\
\hline Iron & $\mu \mathrm{g} / \mathrm{L}$ & 184 & 177 & 3.88 \\
\hline Lead & $\mu \mathrm{g} / \mathrm{L}$ & 13.2 & 13.3 & 0.75 \\
\hline Magnesium & $\mu \mathrm{g} / \mathrm{L}$ & 17,100 & 16,300 & 4.79 \\
\hline Nickel & $\mu \mathrm{g} / \mathrm{L}$ & 1.43 & 0.984 & 36.95 \\
\hline Nitrogen, nitrate/nitrite & $\mathrm{mg} \mathrm{N} / \mathrm{L}$ & 1.04 & 1.04 & 0.00 \\
\hline Potassium & $\mu \mathrm{g} / \mathrm{L}$ & 2,150 & 2,000 & 7.23 \\
\hline Sodium & $\mu \mathrm{g} / \mathrm{L}$ & 7,160 & 7,220 & 0.83 \\
\hline Strontium & $\mu \mathrm{g} / \mathrm{L}$ & 226 & 226 & 0.00 \\
\hline Tritium & $\mathrm{mg} / \mathrm{L}$ & 467 & 435 & 7.10 \\
\hline Uranium & $\mu \mathrm{g} / \mathrm{L}$ & 1.7 & 1.64 & 3.59 \\
\hline Zinc & $\mu \mathrm{g} / \mathrm{L}$ & 161 & 165 & 2.45 \\
\hline \multicolumn{5}{|l|}{ USGS-104 } \\
\hline Alkalinity, total as $\mathrm{CaCO}_{3}$ & $\mathrm{mg} / \mathrm{L}$ & 122 & 121 & 0.82 \\
\hline Barium & $\mu \mathrm{g} / \mathrm{L}$ & 31.5 & 31 & 1.60 \\
\hline Calcium & $\mu \mathrm{g} / \mathrm{L}$ & 35,000 & 33,400 & 4.68 \\
\hline Chloride & $\mathrm{mg} / \mathrm{L}$ & 13.1 & 13 & 0.77 \\
\hline Chromium & $\mu \mathrm{g} / \mathrm{L}$ & 7.62 & 9.91 & 26.13 \\
\hline Fluoride & $\mu \mathrm{g} / \mathrm{L}$ & 0.231 & 0.23 & 0.43 \\
\hline Gross beta & $\mathrm{pCi} / \mathrm{L}$ & 1.62 & 3.35 & 69.62 \\
\hline Iron & $\mu \mathrm{g} / \mathrm{L}$ & 94 & 77.9 & 18.73 \\
\hline Magnesium & $\mu \mathrm{g} / \mathrm{L}$ & 13,300 & 12,500 & 6.20 \\
\hline Nickel & $\mu \mathrm{g} / \mathrm{L}$ & 1.1 & 1.02 & 7.55 \\
\hline Nitrogen, nitrate/nitrite & $\mathrm{mg} \mathrm{N} / \mathrm{L}$ & 0.905 & 0.905 & 0.00 \\
\hline Potassium & $\mu \mathrm{g} / \mathrm{L}$ & 2,640 & 2,500 & 5.45 \\
\hline Sodium & $\mu \mathrm{g} / \mathrm{L}$ & 8,960 & 8,160 & 9.35 \\
\hline Strontium & $\mu \mathrm{g} / \mathrm{L}$ & 209 & 207 & 0.96 \\
\hline Sulfate & $\mathrm{mg} / \mathrm{L}$ & 20.6 & 20.6 & 0.00 \\
\hline Tritium & $\mathrm{pCi} / \mathrm{L}$ & 753 & 567 & 28.18 \\
\hline Uranium & $\mu \mathrm{g} / \mathrm{L}$ & 1.83 & 1.83 & 0.00 \\
\hline
\end{tabular}


Table B-1 shows samples in which the analyte is detected in both the sample and the duplicate. Precision for the data in Table B-1 is acceptable, except for chromium, gross beta, and tritium in Well USGS-104 and cobalt, gross beta, and nickel in Well USGS-106. All unacceptable RPDs were close to the detection limit. Consequently, small differences in concentration near the detection limit can result in a large RPD. However, from the project-data-use perspective, concentration numbers are the same for data interpretation purposes and are, therefore, viewed as acceptable.

\section{B-1.1.2 Overall Accuracy}

Accuracy is a measure of bias in a measurement system. Accuracy is affected by methods used for sample preservation, sample handling, field contamination, and sample matrix. Effects of the first three methods are evaluated using the field blank, trip blank, and equipment rinsate results. The presence of a contaminant in the field blank, trip blank, or rinsate reveals that cross-contamination has occurred.

Laboratory accuracy is ensured through use of standard methods and calibration standards from the National Institute of Standards and Technology. All instrumentation is calibrated before use, in accordance with procedures outlined in the analytical methods required by INL Sample and Analysis Management (SAM) statements of work. Laboratory accuracy is assessed through use of matrix spikes and laboratory control samples. The number of laboratory quality control samples is specified in analytical methods employed in INL SAM statements of work. Evaluation criteria for quality control samples are specified in data validation technical procedures administered by INL SAM. For samples analyzed in accordance with the U.S. Environmental Protection Agency (EPA) Contract Laboratory Program protocol, validation is also performed in accordance with that protocol. Overall accuracy of the analyses is acceptable for the FY 2009 data set.

\section{B-1.1.3 Representativeness}

Representativeness is a qualitative parameter that expresses the degree to which sampling and analysis data accurately and precisely represent the characteristic of a population parameter that drives an action to be taken. Representativeness is evaluated by determining whether measurements were accurate and whether samples represent actual concentrations in the aquifer.

For the FY 2009 sampling activity, all measurements were made according to established EPA and INL SAM protocol. Trained personnel followed established INL procedures to collect physical samples.

\section{B-1.1.4 Comparability}

Comparability is a qualitative characteristic that refers to the confidence with which one data set can be compared to another. At a minimum, comparable data must be obtained using unbiased sampling designs. If sampling designs are biased, the reasons for selecting another design should be well documented. Data comparability for this sampling activity was ensured through the following efforts:

- $\quad$ All data sets contained the same variables of interest

- $\quad$ All measurements were performed and results were reported using common units

- Similar analytical procedures and quality assurance measures were used

- All field and laboratory instrumentation had detection limits that were similar to or better than those historically employed

- $\quad$ Established INL procedures were followed during sample collection

- Wells selected for sampling were identical to those chosen historically. 
Sampling rounds are conducted at approximately the same time of year in an effort to negate any effect that changes in groundwater levels (e.g., caused by snowmelt and runoff) may have on data.

\section{B-1.2 Completeness}

The completeness goal for WAG 10 sampling is 90\%. During the FY 2009 sampling event, 12 of 14 groundwater wells were sampled, and 10 of 10 Westbay intervals were sampled. The completeness was 25 of 27 total samples or $92.6 \%$, thus exceeding the completeness goal of $90 \%$.

\section{B-1.3 Data Validation}

Method data validation is the process whereby analytical data are reviewed against set criteria to ensure that the results conform to requirements of the analytical method and any other specified requirements. Laboratory data for the June-July 2009 sampling event were validated to Level B in accordance with established INL SAM and EPA protocols. The limitations and validation reports were transmitted to the regulatory agencies (i.e., U.S. Department of Energy, EPA, and Idaho Department of Environmental Quality) in October 2009. No major problems were identified during this method validation process.

\section{B-1.4 Performance Evaluation Samples}

Radioanalytical performance evaluation water samples GWA05501RH, GWA05501R8, and GWA05501UX were submitted to GEL Laboratories, LLC, as double-blind performance evaluation samples included with the WAG 10 FY 2009 annual sampling event. Performance evaluation samples were prepared by the U.S. Department of Energy (DOE) Radiological and Environmental Sciences Laboratory (RESL) The goal of performance evaluation samples was to assess the laboratory's proficiency in performing routine radioanalytical measurements.

Radioanalytical results were evaluated using the electronic data system at DOE RESL. For a result to be acceptable, the statistical difference between the laboratory result and the known value must be within 3-sigma, and the laboratory result must be within $\pm 30 \%$ of the known value. The results met acceptance criteria established by DOE RESL, except for tritium and I-129 (Table B-2). The tritium result for GWA05501R8 is evaluated as not acceptable because the statistical difference of the sample result and the known value was greater than 3-sigma, and the laboratory/reference value ratio was not within the acceptance range. The I-129 result for GWA05501UX is evaluated as not acceptable because the statistical difference of the sample result and the known value was greater than 3-sigma, and the laboratory/reference value ratio was not within the acceptance range (i.e., sample result is low-biased).

An investigation of the unacceptable tritium results and a description of the corrective action taken were requested from the GEL Laboratories. The lab determined that the tritium sample probably was not loaded into the scintillation cocktail, which resulted in the original failing to be within the acceptable range for the performance evaluation sample. Consequently, the original performance evaluation sample was shaken and recounted. The reanalysis result yielded an acceptable result. The corrective action identified was for GEL Laboratories to train liquid-scintillation-counting analysts to ensure that samples are shaken sufficiently before counting is performed to ensure that the sample is loaded into the scintillation cocktail. The corrective action training was completed on October 22, 2009. 
GEL Laboratories investigated the unacceptable result for I-129 and found an issue with a detector. The original performance evaluation sample result was biased low. However, a reanalysis of the performance evaluation sample produced a result that was acceptable. The detector used when the low result was obtained was removed from service. The corrective action identified by GEL Laboratories was to train the gamma spectroscopy analysts and associated review staff on pertinent criteria when evaluating a sample. The corrective action training was completed on October 22, 2009.

Table B-2. Performance evaluation sample results for Fiscal Year 2009.

\begin{tabular}{|c|c|c|c|c|c|}
\hline $\begin{array}{c}\text { Performance } \\
\text { Evaluation Sample } \\
\text { Identification } \\
\end{array}$ & Nuclide & $\begin{array}{l}\text { Reference Activity } \\
(\mathrm{pCi} / \mathrm{L})\end{array}$ & $\begin{array}{l}\text { Lab Result }^{\mathrm{a}} \\
(\mathrm{pCi} / \mathrm{L})\end{array}$ & $\begin{array}{c}\text { Ratio } \\
\text { Lab/Ref }^{b}\end{array}$ & Agreement \\
\hline GWA05501RH & Sr-90 & Blank & $2 \pm 9 \mathrm{E}-02$ & - & Yes \\
\hline GWA05501RH & Tc-99 & $2.4 \pm 0.07 \mathrm{E}+01$ & $2.8 \pm 0.3 \mathrm{E}+01$ & 1.16 & Yes \\
\hline GWA05501UX & $\mathrm{I}-129$ & $5.03 \pm 0.12 \mathrm{E} 0$ & $2.66 \pm 0.15 \mathrm{E} 0$ & 0.53 & No \\
\hline Reanalysis & & & $5.08 \pm 0.394 \mathrm{E} 0$ & 1.01 & Yes \\
\hline GWA05501R8 & H-3 & $1.17 \pm 0.03 \mathrm{E}+04$ & $7.8 \pm 0.8 \mathrm{E}+03$ & 0.67 & No \\
\hline Reanalysis & & & $1.42 \pm 0.141 \mathrm{E}+04$ & 1.21 & Yes \\
\hline
\end{tabular}

\section{B-2. REFERENCES}

DOE-ID, 2007, Groundwater Monitoring and Field Sampling Plan for Operable Unit 10-08, DOE/ID-11210, Rev. 2, U.S. Department of Energy Idaho Operations Office, May 2007. 